\title{
A New Spin on Photoemission Spectroscopy
}

\author{
by
}

Christopher Matthew Jozwiak

B.S. (Duke University) 2001

M.A. (University of California, Berkeley) 2005

A dissertation submitted in partial satisfaction of the requirements for the degree of

Doctor of Philosophy

in

Physics

in the

GRADUATE DIVISION

of the

UNIVERSITY OF CALIFORNIA, BERKELEY

Committee in charge:

Professor Alessandra Lanzara, Chair

Professor Dung-Hai Lee

Professor Junqiao Wu

Fall 2008 
The dissertation of Christopher Matthew Jozwiak is approved.

\begin{tabular}{lc}
\hline Chair & Date \\
& \\
& \\
\hline
\end{tabular}

University of California, Berkeley

Fall 2008 
A New Spin on Photoemission Spectroscopy

Copyright @ 2008

by

Christopher Matthew Jozwiak 


\author{
Abstract \\ A New Spin on Photoemission Spectroscopy \\ by \\ Christopher Matthew Jozwiak \\ Doctor of Philosophy in Physics \\ University of California, Berkeley \\ Professor Alessandra Lanzara, Chair
}

The electronic spin degree of freedom is of general fundamental importance to all matter. Understanding its complex roles and behavior in the solid state, particularly in highly correlated and magnetic materials, has grown increasingly desirable as technology demands advanced devices and materials based on ever stricter comprehension and control of the electron spin. However, direct and efficient spin dependent probes of electronic structure are currently lacking.

Angle Resolved Photoemission Spectroscopy (ARPES) has become one of the most successful experimental tools for elucidating solid state electronic structures, bolstered by continual breakthroughs in efficient instrumentation. In contrast, spin-resolved photoemission spectroscopy has lagged behind due to a lack of similar instrumental advances. The power of photoemission spectroscopy and the pertinence of electronic spin in the current research climate combine to make breakthroughs in Spin and Angle Resolved Photoemission Spectroscopy (SARPES) a high priority .

This thesis details the development of a unique instrument for efficient SARPES and represents a radical departure from conventional methods. A custom designed spin polarimeter based on low energy exchange scattering is developed, with projected efficiency gains of two orders of magnitude over current state-of-the-art polarimeters. For energy analysis, the 
popular hemispherical analyzer is eschewed for a custom Time-of-Flight (TOF) analyzer offering an additional order of magnitude gain in efficiency. The combined instrument signifies the breakthrough needed to perform the high resolution SARPES experiments necessary for untangling the complex spin-dependent electronic structures central to today's condensed matter physics.

Chapter 1 provides an introduction to the technique with background on electron spin, its important interactions, and the benefits of spin-resolved experiments. Chapter 2 contains an in-depth review of ARPES, and Chapter 3 reviews the addition of spin-resolution. Chapters 4 and 5 detail the full development of the new SARPES instrument, and Chapter 6 presents the complete spectrometer and its initial promising results. Chapter 7 gives a brief conclusion with a discussion of future directions.

Professor Alessandra Lanzara

Dissertation Committee Chair 
To my family and especially my wife. 


\section{Contents}

$\begin{array}{ll}\text { Abstract } & 1\end{array}$

Contents $\quad$ v

List of Figures $\quad$ vi

Acknowledgments $\quad$ ix

Curriculum vitae xiii

1 Introduction 1

1.1 Expansion of electron spectroscopy . . . . . . . . . . . . . . 1

1.2 Spin - the ups and downs of being an electron . . . . . . . . . . . . . . 3

1.2.1 Almost discovery - the Stern-Gerlach experiment . . . . . . . . 3

1.2.2 Discovery - Uhlenbeck and Goudsmit . . . . . . . . . . . 8

1.2 .3 Spin-orbit coupling . . . . . . . . . . . . . . . 11

1.2 .4 Symmetry of spin . . . . . . . . . . . . . . . . . . . . . 14

1.2 .5 Exchange coupling . . . . . . . . . . . . . . . . . 18

1.3 Probing spin in solids . . . . . . . . . . . . . . . . . 23

2 Photoemission Spectroscopy 26

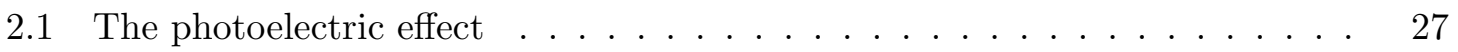

2.2 Energy resolution . . . . . . . . . . . . . . . . . . . . . 32

2.3 Angular resolution . . . . . . . . . . . . . . . . . 36

2.4 Single particle spectral function . . . . . . . . . . . . . 43

2.5 EDC and MDC analysis . . . . . . . . . . . . . 47

2.6 Dipole matrix elements . . . . . . . . . . . . . . . . . . 48 
2.7 State-of-the-art ARPES . . . . . . . . . . . . . . . . . 50

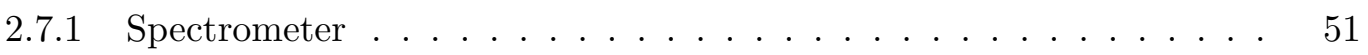

2.7 .2 Ultra-high vacuum system . . . . . . . . . . . . . . 61

2.7 .3 Sample manipulation . . . . . . . . . . . . . . . . . 65

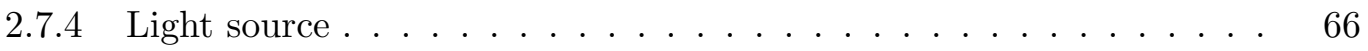

3 Spin-Resolved Photoemission Spectroscopy $\quad 69$

3.1 Three-step model revisited . . . . . . . . . . . . . . . . . . . . . 70

$3.1 .1 \quad$ Step one . . . . . . . . . . . . . . . . . 70

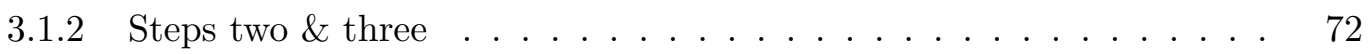

3.2 Spin resolved PES from non-magnetic samples _ . . . . . . . . . 74

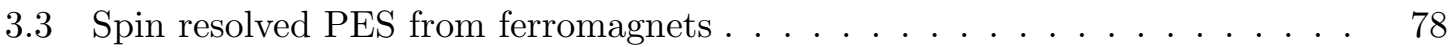

3.4 Current state-of-the-art technique . . . . . . . . . . . . . . . . 80

3.5 Novel approach . . . . . . . . . . . . . . . . . . . . . 85

4 Time of Flight Spectroscopy $\quad 88$

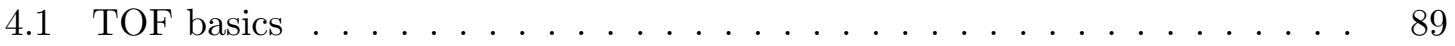

4.2 TOF advantages $\ldots \ldots \ldots \ldots \ldots \ldots \ldots \ldots \ldots \ldots$

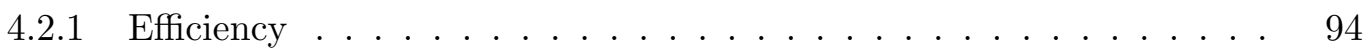

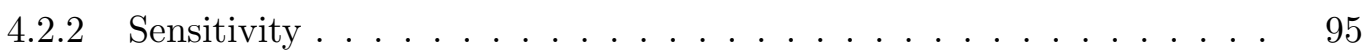

4.2 .3 Ultimate resolution . . . . . . . . . . . . . . . . . . 96

4.3 Previous uses of TOF $\ldots \ldots \ldots \ldots \ldots \ldots \ldots \ldots \ldots$

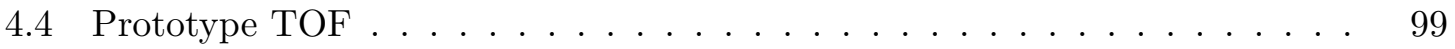

$4.4 .1 \quad \mathrm{MCP}$ detector . . . . . . . . . . . . . . . . . . . . 99

4.4 .2 Timing electronics $\ldots \ldots \ldots \ldots \ldots \ldots \ldots$

4.4 .3 Lens column . . . . . . . . . . . . . . . . . . . . . . . . . 109

4.4.4 Beamtime, data acquisition, and time-to-energy conversion . . . . 111

4.4.5 A few remarks on TOF overlap . . . . . . . . . . . . . . . 119

4.5 Electrostatic lens system . . . . . . . . . . . . . . . . . . 121

4.5 .1 General design considerations . . . . . . . . . . . . . . 121

4.5.2 Magnetic shielding . . . . . . . . . . . . . . . . . . . . . 124

4.5.3 Mechanical and material considerations . . . . . . . . . . . 128

4.5 .4 Assembly and inspection . . . . . . . . . . . . . . . . . . . 131

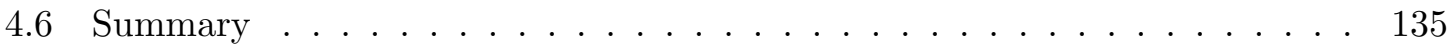


5 Spin Polarimetry $\quad 136$

5.1 Spin Polarization . . . . . . . . . . . . . . . . . . . 136

5.2 Spin-orbit polarimetry . . . . . . . . . . . . . . . . . . . . . 142

5.2 .1 Mott scattering . . . . . . . . . . . . . . . . . . . . . 142

5.2 .2 Polarimeter efficiency . . . . . . . . . . . . . . . . 146

5.2.3 Mott polarimeter design and usage . . . . . . . . . . . . . . . . 148

5.2 .4 Other techniques . . . . . . . . . . . . . . . 151

5.3 Exchange polarimetry . . . . . . . . . . . . . . . 154

5.3 .1 Exchange scattering . . . . . . . . . . . . . . 154

5.3.2 Qualitative understanding of exchange asymmetry . . . . . . . 159

5.3.3 More details: spin-orbit contributions . . . . . . . . . . . . . . . 160

5.3.4 More details: instrumental asymmetry . . . . . . . . . . . . . . . . 164

5.3.5 Previous exchange polarimeters . . . . . . . . . . . . 166

5.4 New exchange polarimeter . . . . . . . . . . . . . . . 167

5.4 .1 Target characterization . . . . . . . . . . . . 168

5.4 .2 Scattering geometry and detection . . . . . . . . . . . 174

5.4 .3 Full polarimeter layout . . . . . . . . . . . . . . . . . . 179

5.4.4 Target manipulation and magnetization . . . . . . . . . . . . . 181

5.5 Summary . . . . . . . . . . . . . . . . . . 185

6 SARPES with the spin-TOF spectrometer $\quad 186$

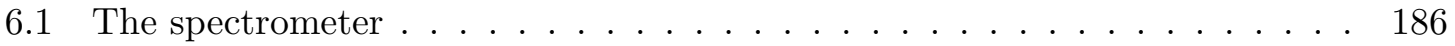

6.1.1 Combining the TOF lens system and exchange polarimeter . . . . 186

6.1 .2 Instrumental control . . . . . . . . . . . . . . . . . . . . 190

6.1.3 Experimental endstation . . . . . . . . . . . . . . . 193

6.1 .4 Bandpass filter details . . . . . . . . . . . . . . . 193

6.2 Spin-resolved ARPES _. . . . . . . . . . . . . . . 197

6.2 .1 Sample preparation $\ldots \ldots \ldots \ldots$. . . . . . . . . . 197

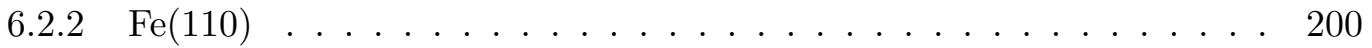

$6.2 .3 \mathrm{Au}(111)$ surface state $\ldots \ldots \ldots \ldots \ldots$

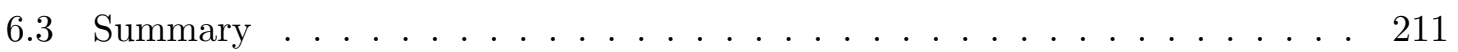

7 Outlook, applications, and conclusions $\quad 213$

7.1 Future outlook . . . . . . . . . . . . . . . . . . . . 213 
7.2 Exciting applications . . . . . . . . . . . . . . . . . . . . . 214

7.2 .1 CMR manganites . . . . . . . . . . . . . . . . . 215

7.2 .2 Rashba effect . . . . . . . . . . . . . . . . . . . . 219

7.2.3 Time-resolved spin dynamics and phase transitions . . . . . . . . . . 221

7.3 Conclusions . . . . . . . . . . . . . . . . . . . . . . . 222

$\begin{array}{lr}\text { Bibliography } & 224\end{array}$ 


\section{List of Figures}

1.1 Angular momentum and space quantization . . . . . . . . . . . 4

1.2 The Stern-Gerlach experiment . . . . . . . . . . . . . 6

1.3 SGE original results $\ldots \ldots \ldots \ldots \ldots \ldots \ldots$

1.4 Identical particles . . . . . . . . . . . . . . . . . . . . . 15

2.1 Hertz's photoelectric effect . . . . . . . . . . . . . . . 28

2.2 Early photoemission instruments of Lenard and Millikan . . . . . . . . . . 29

2.3 Early photoemission experiment f . . . . . . . . . . . . . 31

2.4 Energy diagram of photoemission spectroscopy $\ldots \ldots$. . . . . . . . . 33

2.5 Examples of Siegbahn's ESCA _ . . . . . . . . . . . . . . 35

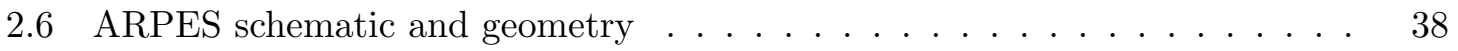

2.7 Universal curve of electron mean free paths . . . . . . . . . . . . . . 39

2.8 Example of ARPES bandmapping . . . . . . . . . . . . . 42

2.9 Spectral function picture of ARPES . . . . . . . . . . . 46

2.10 EDC and MDC ARPES analysis . . . . . . . . . . . . . . 48

2.11 Hemispherical analyzer schematic . . . . . . . . . . . . . . . 53

2.12 Operation of a hemispherical analyzer f . . . . . . . . . . . 57

2.13 Data acquisition in 3D phase space . . . . . . . . . . . 59

2.14 ARPES resolution improvements . . . . . . . . . . . . . . . 60

2.15 ARPES endstation layout . . . . . . . . . . . . . . 63

2.16 Endstation load-lock and sample storage carousels . . . . . . . . . . 64

2.17 Endstation sample goniometer and cryostat . . . . . . . . . . 65

2.18 Endstation photograph . . . . . . . . . . . . . . 68

3.1 Spin polarized photoemission from GaAs . . . . . . . . . . 75 
3.2 Spin-resolved resonant photoemission spectra from $\mathrm{Bi}_{2} \mathrm{Sr}_{2} \mathrm{CaCu}_{2} \mathrm{O}_{8+\delta} \quad \ldots \quad$. 77

3.3 Spin-resolved ARPES from $\mathrm{Fe}(100)$ and $\mathrm{Gd}(0001) \ldots \ldots$

3.4 Principles of current state-of-the-art approach to spin-ARPES . . . . . . . 82

3.5 High impact photoemission experiment due to moderate-high resolutions . . 84

4.1 Schematic of TOF technique . . . . . . . . . . . . . . 90

4.2 Dependence of TOF energy resolution on kinetic energy . . . . . . . . . . . 92

4.3 Multichannel plates (MCPs) . . . . . . . . . . . . . . . . . . . 100

4.4 MCP detector photographs . . . . . . . . . . . . . . 103

4.5 Signal from MCP detector . . . . . . . . . . . . . . . . . 104

4.6 Timing signal triggering techniques . . . . . . . . . . . . 106

4.7 Timing resolution measurement . . . . . . . . . . . . . . . 108

4.8 TOF prototype lens column photograph . . . . . . . . . . . . . . . 110

4.9 Beamspot photograph . . . . . . . . . . . . . . . . . . . . . 112

4.10 Schematic layout of TOF photoemission experiment f . . . . . . . . 113

4.11 TOF time spectrum of Au photoemission . . . . . . . . . . . . . 115

4.12 Time to energy conversion . . . . . . . . . . . . . . 116

4.13 TOF energy spectrum of Au photoemission . . . . . . . . . . . 116

4.14 TOF Au Fermi edge . . . . . . . . . . . . . . . . . 117

4.15 Sectioned view of TOF Lens System . . . . . . . . . . . . . 122

$4.16 \mu$-metal magnetic shielding . . . . . . . . . . . . . 125

4.17 Electron flight paths in a magnetic field . . . . . . . . . . . . . 126

4.18 Photograph of ruby ball electrode spacers . . . . . . . . . . . . . . . . . 129

4.19 Photographs of lens system assembly . . . . . . . . . . . . . . . 132

4.20 Photographs of BPF exit slit assembly . . . . . . . . . . . . . 133

4.21 Photograph of mostly assembled TOF lens system . . . . . . . . . . . . 134

5.1 Spin-orbit scattering potential and geometry f . . . . . . . . . . . 143

5.2 Mott polarimeter working principles . . . . . . . . . . . . . 145

5.3 Mott polarimeter diagram and usage schematic . . . . . . . . . . . . . 150

5.4 Diagram of LEED polarimeter spectrometer . . . . . . . . . . . . 153

5.5 Exchange scattering potential and geometry . . . . . . . . . . 155

5.6 Exchange polarimeter working principles . . . . . . . . . . 157

5.7 Spin asymmetry of Fe electron reflectivity . . . . . . . . . . . . 159 
5.8 Particularly useful scattering geometries . . . . . . . . . . . . . . . 161

5.9 SPLEEM diagram and reflectivity data . . . . . . . . . . . . . . 169

5.10 Quantum size effects in thin film reflectivity . . . . . . . . . . . 171

5.11 Spin asymmetry in reflectivity measured by SPLEEM . . . . . . . . . . . 172

5.12 Sherman functions and FOMs in 2D phase space . . . . . . . . . . . . . 174

5.13 Diagram of polarimeter scattering and detection design . . . . . . . . . 175

5.14 Photographs of polarimeter MCP detector assembly . . . . . . . . . 177

5.15 Signal and timing resolution from polarimeter detector . . . . . . . . . 178

5.16 Cross sectional diagram of spin polarimeter . . . . . . . . . . . . . 180

5.17 Photographs of polarimeter prep chamber components . . . . . . . . . 181

5.18 Photographs of target stage and magnetization coils . . . . . . . . . . 182

6.1 Cross sectional diagram of spin-TOF spectrometer $\ldots \ldots \ldots$. . . . . 187

6.2 Power supply software . . . . . . . . . . . . . . . . . . . . . . . 190

6.3 Spin-TOF wiring diagram . . . . . . . . . . . . . . . . . . . . . 192

6.4 Photograph of spin-TOF and endstation at beamline 12.0.1 . . . . . . . . 194

6.5 Electron gun investigation of bandpass filter . . . . . . . . . . . . . 196

6.6 Mode \#1 spectra of clean W surface . . . . . . . . . . . . . . . . . 198

6.7 Previous SARPES data from $\mathrm{Fe}(110) \ldots \ldots \ldots$. . . . . . . . . 201

6.8 Mode \#2 spectrum from $\mathrm{Fe}(110) \ldots \ldots$. . . . . . . . . . . . . 202

6.9 Mode \#2 spin-resolved spectrum from $\mathrm{Fe}(110) \ldots \ldots$. . . . . . . . . 203

6.10 More spin-resolved spectra of $\mathrm{Fe}(110) \ldots \ldots \ldots$

6.11 Spin-polarized $\mathrm{Au}(111)$ surface state . . . . . . . . . . . . . . . . . . 209

6.12 Spin-resolved spectra of $\mathrm{Au}(111)$, previous and with spin-TOF . . . . . . 210

7.1 New ARPES results from bilayer CMR manganites . . . . . . . . . . . . 216

7.2 Previous spin-resolved photoemission from CMR manganites . . . . . . . 218

7.3 Rashba spin-splitting in graphene? . . . . . . . . . . . . . . . . . . 220

7.4 Time-resolved experiments in ultra-fast CMR phase transitions . . . . . . . 221 


\section{Acknowledgments}

I have been very lucky to work on my Ph.D. surrounded by such a large group of talented and kind people. It is an understatement to say that the work presented in this thesis would not have been possible without them. I am not sure their help and contributions can be thanked enough.

First of all, I would like to thank my advisor, Alessandra Lanzara, for all that she does for our group. I consider myself very lucky to have joined her group where she has provided me a vast amount of trust and patience. She has allowed me to learn, make mistakes, and develop these projects as I saw fit. The long (and ongoing!) projects I have worked on have certainly required her patience and constant commitment to see them through, which she has always generously supplied. Somehow she is able to know when to step back and let things happen, and when to step in and give much appreciated guidance and encouragement. I'd like to thank Alessandra for the support, advice, and training I needed to complete this Ph.D., and additionally for always looking out for our best interests in the group, in science and beyond.

I owe many special thanks to Zahid Hussain. Leading the spin-TOF project, he has brought together a great team of experts to help make it happen and has always provided the help, direction, and funds required to push it forwards. This project is really due to his scientific creativity, energy, and leadership. I have been very lucky to get to work with him. Like Alessandra, he placed a large amount of trust in both the project and me, and I am indebted to him for both his patience and mentorship. Somehow he always makes time for my questions and updates (both good and bad), even when I track him down in his office late on Friday afternoons.

Andreas Schmid has been of particular help in many ways. Not only did he teach me an incredible amount of experimental science technique in my first years in his lab, he has continued to be a reliable source of advice and eerily correct intuition about almost anything. Leader of the SPLEEM lab, he is also the source of much of the spin-polarimeter 
design and other clever ideas found throughout the project. I don't know where we would be without our close collaborations with Andreas.

I don't think it is possible to go through a Ph.D. without having others to go through it with together. I have been very lucky to go through it with the other students in the Lanzara group. Dr. Jeff Graf and Dr. Shuyun Zhou (both soundly beat me to the finish line!) started with me as the first members of the group, and I am indebted to them for the majority of our Ph.D. memories. They both set the bar extremely high for our group, and yet were always willing to help and explain as they quickly learned. Jeff has played a major role in the development of the lab equipment and the spin-TOF project, which could not have been completed without his insights or willingness to spend the time to understand the problems at hand and point out where my mistakes were. I think we made a great team in attacking the constant problems of the lab. The amount of great science that Shuyun was able to accomplish was (and is) quite inspiring. She was a constant source of friendly help, and her leadership by example was greatly missed as soon as she left the group. It has also been a pleasure to work and learn alongside Daniel Garcia and David Siegel and see them progress so quickly. The group's postdocs have also been invaluable sources of help. Professor Gey-Hong Gweon taught me the fundamentals of photoemission experiments and the importance of attentive precision in both taking and interpreting data. The impact Professor Kyle McElroy had on our group in such a short stay speaks volumes about his skills as a scientist and person. Finally, Dr. Choonkyu Hwang has been great to work with and learn from.

The ALS community as a whole has been a wonderful place to study. I'd like to thank the entire community for providing so many opportunities and so much help. With such a large group of experts, one has to try really hard to not learn something simply by walking around the ring. I have learned so much from so many at the ALS, it is impossible to thank everyone. I certainly have spent the most time harassing everyone in Eli Rotenberg's group at beamline 7. In particular, Eli, Aaron Bostwick, and Jessica McChesney were great sources of help, borrowed equipment, and usually both. An absurd amount of what I learned in my time here came from Aaron; how he managed to learn everything I have no 
idea. Alexei Fedorov has also provided much valuable advice and wisdom, and has helped a great deal with the measurements taken at beamline 12.0.1.

The spin-TOF project has only been possible with the creativity and expertise of a wide range of people at the ALS. Nord Andresen produced most of the mechanical design, and he taught me quite a bit about mechanical design and CAD techniques. The electrostatic lens system was designed by Gennadi Lebedev, who has been a central figure in the project. Much help with the MCP detectors came from Ali Belkacem and Marc Hertlein. Yulin Chen has been a constant source of expertise and help, both at the beamline and with general advice, always making time even as he worked on developing his own spin-resolved system. And thanks to Professor Dung-Hai Lee for friendly and insightful theoretical input.

The ALS runs in large part because of its large base of skilled technicians, machinists, mechanics, and electricians. The large amount of instrumental development in my Ph.D. allowed me to work with and learn a great deal from a large number of them. First and foremost, John Pepper has been an integral part of all the equipment in our lab, including the spin-TOF. He has taught me an inordinate amount about design and machining and in general what makes things work and (more interestingly) not work. He and his group members, Scott DiMaggio and Adrian Williams, and the rest of the building 80 machine shop provided countless last second critical parts. The entire Vac-Tec group has been invaluable with wide range of vacuum and equipment issues. Much of the spin-TOF equipment was fabricated in building 77; I'd like to thank everyone there, including Ed Tully, Paul Knopp, Bob Conroy, and Kit Man Mui for their exceptional work.

Finally I thank my amazing wife, Erin, for the enormous support she has given over a very long time. Although it makes it hard to stay at the lab late when there is such great company at home, I wouldn't have made it without her. Thanks, Erin. I'd also like to thank my parents and the rest of my fantastic and growing family and friends for all their love and support - you guys know each other. Thanks for everything!

This work was supported by and performed at the Advanced Light Source, Lawrence Berkeley National Laboratory, which is supported by the Director, Office of Science, Office 
of Basic Energy Sciences, of the U.S. Department of Energy under Contract No. DE-AC0205CH11231. This work was also supported by the Director, Office of Science, Office of Basic Energy Sciences, Materials Sciences and Engineering Division, of the U.S. Department of Energy under Contract No. DE-AC02-05CH11231. Additional funding support was given by Professor Lanzara's start-up funds provided by the University of California, Berkeley.

Chris Jozwiak December 9, 2008 


\section{Curriculum Vitæ}

Christopher Matthew Jozwiak

\section{Education}

2008 University of California, Berkeley, CA, USA

Ph.D., Physics

2005 University of California, Berkeley, CA, USA

M.A., Physics

2001 Duke University, Durham, North Carolina, USA

B.S., Physics

\section{Honors and Awards}

2006 UC Berkeley Nano-science Research Fellowship

\section{Publications}

$1 \quad$ Bilayer splitting and c-axis coupling in CMR bilayer manganites.

C. Jozwiak, J. Graf, S.Y. Zhou, A. Bostwick, E. Rotenberg, H. Zheng, J.F. Mitchell, and A. Lanzara

In review, Phys. Rev. B

2 Core level and valence band study of $\mathrm{LaFeAsO}_{0.9} \mathrm{~F}_{0.1}$.

D.R. Garcia, C. Jozwiak, C.G. Hwang, A. Fedorov, S.M. Hanrahan, S.D. Wilson, C.R. Rotundu, B.K. Freelon, R.J. Birgeneau, E. Bourret-Courchesne, and A. Lanzara.

To be published Phys. Rev. B (2008)

3 TOF electron energy analyzer for spin and angular resolved photoemission spectroscopy.

G. Lebedev, C. Jozwiak, N. Andresen, A. Lanzara, and Z. Hussain Physics Procedia 1, 413 (2008). 
Bond stretching phonon softening and angle-resolved photoemission kinks in optimally doped $\mathrm{Bi}_{2} \mathrm{Sr}_{1.6} \mathrm{La}_{0.4} \mathrm{Cu}_{2} \mathrm{O}_{6+\delta}$.

J. Graf, M. d'Astuto, C. Jozwiak, D.R. Garcia, N.L. Saini, M. Krisch, K. Ikeuchi, A.Q.R. Baron, H. Eisaki, and A. Lanzara

Phys. Rev. Lett. 100, 227002 (2008).

$5 \quad$ High spatial and temporal resolution photon/electron counting detector for synchrotron radiation research.

A.S. Tremsin, G.V. Lebedev, O.H.W. Siegmund, J.V. Vallerga, J.S. Hull, J.B. McPhate, C. Jozwiak, Y. Chen, J.H. Guo, Z.X. Shen, and Z. Hussain

Nuc. Instr. Meth. Phys. Res. A 580, 853 (2007).

6 Revealing charge density wave formation in the $\mathrm{LaTe}_{2}$ system by angle-resolved photoemission spectroscopy.

D.R. Garcia, G.-H. Gweon, S.Y. Zhou, J. Graf, C.M. Jozwiak, M.H. Jung, Y.S. Kwon, and A. Lanzara

Phys. Rev. Lett. 98, 166403 (2007).

$7 \quad$ Universal high energy anomaly in the angle-resolved photoemission spectra of high temperature superconductors: Possible evidence of spinon and holon branches.

J. Graf, G.-H. Gweon, K. McElroy, S.Y. Zhou, C. Jozwiak, E. Rotenberg, A. Bill, T. Sasagawa, H. Eisaki, S. Uchida, H. Takagi, D.-H. Lee, and A. Lanzara

Phys. Rev. Lett. 98, 067004 (2007).

8 MERLIN - A meV resolution beamline at the ALS.

Ruben Reininger, John Bozek, Yi-De Chuang, Malcolm Howells, Nicholas Kelez, Soren Prestemon, Steve Marks, Tony Warwick, Chris Jozwiak, Alessandra Lanzara, M. Zahid Hasan, Zahid Hussain

Sync. Radiation. Instrum. : AIP Conf. Proc. 879, 509 (2007).

$9 \quad$ Mapping spin-dependent electron reflectivity in Fe and Co ferromagnetic thin films.

J. Graf, C. Jozwiak, A.K. Schmid, Z. Hussain and A. Lanzara

Phys. Rev. B 71, 144429 (2005). 
10 Perpendicular Magnetic Coupling in $\mathrm{Fe} / \mathrm{NiO} / \mathrm{Fe}(001)$ Trilayers Studied by Spin Polarized Low Energy Electron Microscopy.

M. Portalupi, G. H. Gweon, C. Jozwiak, J. Graf, A. K. Schmid and A. Lanzara

Journal of Microscopy and Microanalysis 10, 508 (2004).

\section{Invited talks and seminars}

1 "Development of a time-of-flight spin resolved photoemission spectrometer -and the need for a good acronym"

ALS-SSG technical meeting, Lawrence Berkeley National Lab, Feb. 2007

2 "Proposal of an ARPES endstation for the new meV resolution beamline"

ALS User's Meeting, "Frontiers in inelastic X-ray scattering and ultra-high resolution photoemission studies of strongly correlated electron systems" workshop, Lawrence Berkeley National Lab, Oct. 2003

\section{Contributed talks}

1 "Bilayer splitting and c-axis coupling: doping and temperature dependence" American Physical Society March Meeting, New Orleans, LA, March 2008 


\section{Chapter 1}

\section{Introduction}

No experiment is so dumb that it should not be tried.

- Edgar Meyer / Walther Gerlach ${ }^{1,2}$

\subsection{Expansion of electron spectroscopy}

Bound electrons are absolutely essential components of any condensed matter system. They are not only required for its existence due to the local bonding afforded by the electrons, but are also essential for the chemical, physical, vibrational, and transport properties of a material. Especially interesting phenomena, such as magnetism, emerge from the spin of the bound electrons. Measuring, scrutinizing, tweaking, and otherwise probing a system's electronic structure through a wide array of electron spectroscopies have proven fruitful in furthering our understanding of materials and their electrons. In the solid state, electron spectroscopies do not only aid in exploring bulk electronic behavior, but due to their inherent surface sensitivity are particularly well suited for studying surface physics. The current trend in material technology towards ever decreasing dimensions with advancements in, for example, thin films and nanoparticles, enhances the importance of surfaces. The popularity of electron spectroscopies has likewise surged. Interest in the magnetic properties and spin 
dependence of such systems, driven by the promise of advanced electronics and information technologies, has increased the need for spin-sensitive electron spectroscopies.

The technique of photoemission spectroscopy (PES), in which the electrons of interest are ejected out of a sample by impinging photons, is a particularly powerful tool for measuring electronic structure directly. The angle resolved technique (ARPES) is performed by measuring the distribution of photo-ejected electrons with respect to kinetic energy and angle about the sample surface normal. Because energy and momentum parallel to the surface is conserved through the emission process, the binding energy and momentum dependence of the electronic excitations within the sample is directly imaged. Performed with high energy and angular resolutions, ARPES is able to measure details of the electronic structure such as the Fermi surface and electron complex self-energies. ARPES is thus unique in its ability to probe the valence electrons and their energy and momentum dependent bandstructure and correlated behaviors. In this way, ARPES has played a fundamental role in critical avenues of progress in condensed matter physics.

Electron spin is largely conserved in the photoemission process such that resolving photoelectron spin polarization can yield additional information. An uneven distribution of spin in the collected photoelectrons can be reflective of an initial uneven distribution of spin in the material (in the case of ferromagnetic samples, the surfaces of some antiferromagnets, or electronic states split by spin-orbit coupling), or can result from spin-dependent photoemission selection rules when using circularly polarized light. In any case, spin resolved PES experiments offer key insights into a material's spin dependent electronic structure, have already provided great success stories, and give the promise of many more untold discoveries. Progress with all spin-resolved spectroscopies remains slow, however, largely due to instrumental difficulties limiting efficiencies and resolutions. Extension of ARPES with the addition of spin-resolution is an exciting direction to take in the current research climate, but requires advancements in instrumentation to deliver on its vast potential. This thesis covers the complete design, fabrication, and initial uses of a radically unique instrument called the spin time-of-flight, or spin-TOF, a spectrometer with vastly improved detection efficiency which can revolutionize the field of spin-ARPES. 


\subsection{Spin - the ups and downs of being an electron}

It is probably not possible to overestimate the breadth of consequences the electron spin, or intrinsic angular momentum, has on our daily lives. This is surprising as there are only ever two possibilities: an electron must be either "spin-up" or "spin-down", with no in-betweens. With only two possible states, electron spin is the simplest degree of freedom possible, and yet, along with certain rules which Nature follows with respect to spin, the electron's spin makes possible the structure of the Periodic Table. It is also a key component of magnetism in matter which allows a impressive array of daily technologies including electric motors, generators, the hard-drives which house all the backup copies of this thesis, and even the reliable refrigerator magnet. Therefore it is interesting and well worthwhile to review the basics of electron spin physics.

\subsubsection{Almost discovery - the Stern-Gerlach experiment}

The binary nature of electron spin is rooted in the basics of quantum mechanics, so it is not surprising that the history of its discovery is thoroughly entangled with the turbulent evolution of early quantum theory. One of the pillars of quantum physics is the quantization of a particle's possible angular momentum into discreet units, nowadays expressed as:

$$
|\mathbf{L}|=\hbar \sqrt{l(l+1)}
$$

where $l$ is constrained to be a non-negative integer. An additional rule referred to as "space quantization" requires the component of the angular momentum vector along any particular axis to fall in discreet units of the magnitude of Planck's constant, $\hbar=1.055 \times 10^{-34} \mathrm{~J} \cdot \mathrm{s}$, according to:

$$
L_{z}=m_{L} \hbar
$$

where $m_{L}$ can be an integer from $l$ to $-l$. This holds for any axis chosen; the $z$-axis is not particularly special, and equation 1.2 is just as valid written for $L_{x}$ or $L_{y}$. Often times the system under consideration provides a natural axis to reference direction to, such as an applied magnetic field vector, where the physical interaction is given by the component 
(a)

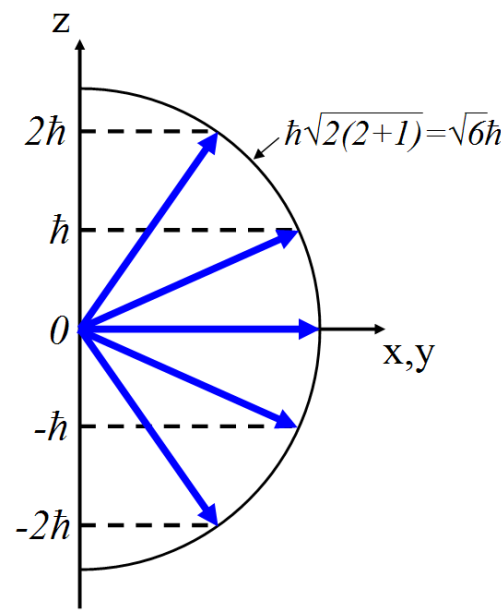

$l=2$ (b)

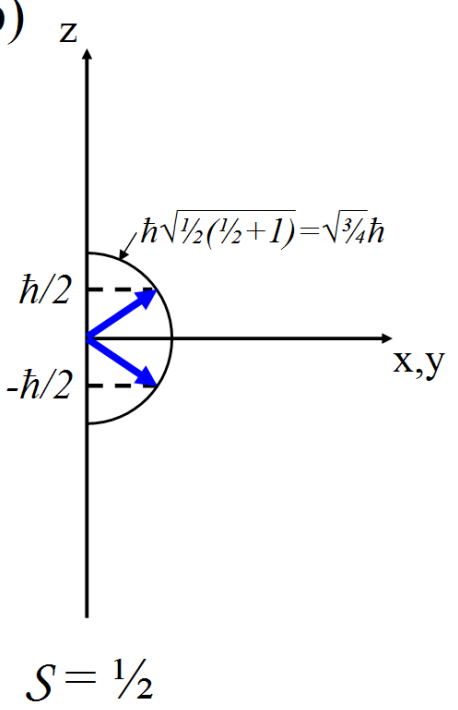

Figure 1.1. Schematic of angular momentum vector directions due to space quantization. (a) The $l=2$ case. (b) The $s=1 / 2$ case, an example of the same rules being applied to intrinsic angular momentum.

of angular momentum along that axis. In this case it is most useful to use equation 1.2 defined along that axis, and by convention it is usually labeled the $z$-axis. The result of these rules for a particle with the quantum number $l=2$ are diagrammed in Figure 1.1(a). Equation 1.1 requires the $l=2$ state to have an angular momentum vector, $\mathbf{L}$, to have a length of $\hbar \sqrt{6}$. Equation 1.2 requires this vector to be oriented in space such that its component along the given $z$-axis can only be $-2 \hbar,-\hbar, 0, \hbar$, or $2 \hbar$. With this picture we see that the term "space quantization" does not mean quantizing actual space in this case, but quantizing the directions $\mathbf{L}$ can have in space.

Quantizing angular momentum in a way similar to Equation 1.1 had much experimental support from agreement between the resulting Bohr model of the atom and studies of atomic emission spectra which formed discreet lines. Only allowing discreet orientations of the angular momenta was harder to believe, and many in the early 1920's did not. Most thought of it as a type of book-keeping which helped explain experiments but should not be taken literally. This thought is expressed in the autobiography of Max Born: "It took me quite a time before I took this idea seriously. I thought always that [space] quantization was a kind of symbolic expression for something which you didn't understand." ${ }^{3}$ In 1921 , 
Otto Stern proposed a method ${ }^{4}$ to experimentally determine whether space quantization was a reality of nature or not. The method relied on the interaction of an orbiting electron and a magnetic field.

Just as the current in a macroscopic loop of wire creates a magnetic dipole, the orbit of an electron also has a magnetic moment

$$
\boldsymbol{\mu}=\mu_{B}(\mathbf{L} / \hbar)
$$

with

$$
\mu_{B}=\frac{e \hbar}{2 m_{e} c}
$$

expressed in Gaussian cgs units, where $m_{e}$ is the electron mass, $c$ is the speed of light in vacuum, and $e$ is the charge of the electron (a negative number). The potential energy $U$ of a magnetic moment, $\boldsymbol{\mu}$, in an external magnetic field, $\mathbf{B}$ is

$$
U=-\boldsymbol{\mu} \cdot \mathbf{B}
$$

whereby the moment will experience a net force

$$
\mathbf{F}=-\nabla U
$$

If this magnetic moment is made to pass through an inhomogeneous magnetic field along the z-axis with a strong gradient along the z-axis, the moment will then feel a force along $\hat{\mathbf{z}}$ of

$$
F_{z} \simeq \mu_{z} \frac{\partial B_{z}}{\partial z}
$$

if we ignore the components of $\mathbf{B}$ along the other directions.

Now we can see Stern's idea. Due to the orbiting motion of an atoms electrons, an atom will posses a magnetic moment given by equation 1.3 with $\mathbf{L}$ being the total angular momentum of the electrons. A beam of such atoms may be setup to pass through an inhomogeneous magnetic field along the $z$-direction. If space quantization is valid, then this magnetic moment will have quantized $z$-components (eqn. 1.3 and eqn. 1.2) and will experience a quantized force in the $z$-direction (eqn. 1.7) while in the field, and then be split into separate, distinct beams based on the $m_{L}$ value of each atom. If space quantization is 
(a)

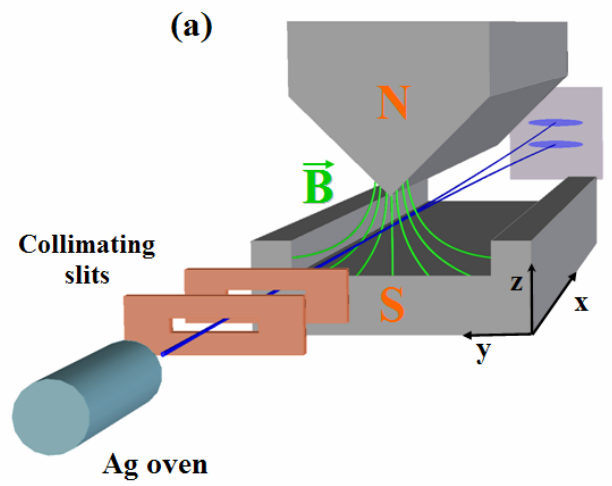

(b)

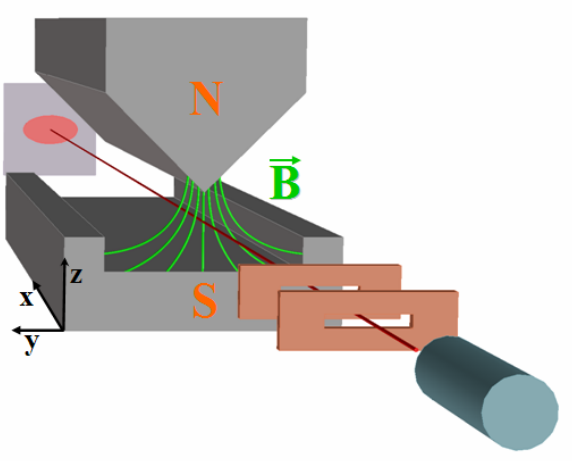

Figure 1.2. Layout of the Stern-Gerlach experiment. Atomic Ag beam is collimated before traveling down the length of a magnet with a strongly inhomogeneous field along the $\hat{\mathbf{z}}$. (a) If the spatial components of angular momentum are quantized, the beam will be split into distinct beams along $\hat{\mathbf{z}}$, resulting in separate spots at the rear detection plane. (b) If angular momentum is classically distributed in space, the beam will be evenly spread in $\hat{\mathbf{z}}$.

not valid, the orientation of the atom's total $\mathbf{L}$, and hence $\boldsymbol{\mu}$ will be randomly oriented in space, producing a smooth distribution of forces in the beam and a continuous spread of the beam along the $z$-axis. A schematic of the experiment and these two possible results are shown in Figure 1.2.

Stern was already an expert at creating atomic beams. Since he wanted as strongly an inhomogeneous field as possible, he recruited Walther Gerlach, who had experience making such magnets, to help. The original experiment they performed used atomic beams of Ag. At the time, the angular momentum quantization rules were not written exactly as in equations 1.1 and 1.2. The Sommerfeld-Bohr model predicted that the total angular momentum of a silver atom would be equivalent to an $l=1$ state, but only allowed for $m_{L}= \pm 1$ without a $m_{l}=0$ value. Thus, the then current theory of space quantization predicted that the orbital angular momentum would split a $\mathrm{Ag}$ beam into two, with a magnitude of splitting corresponding to a difference in magnetic moment along the z-axis of $2 \mu_{B}$.

Stern and Gerlach developed their experiment using Ag atoms expelled from an oven at $1000^{\circ} \mathrm{C}$ and collimated by two slits of $30 \mu \mathrm{m}$ width. The magnet used was $3.5 \mathrm{~cm}$ long and produced a field of approximately $0.1 \mathrm{~T}$ with a gradient of $10 \mathrm{~T} / \mathrm{cm}$. The experiment 


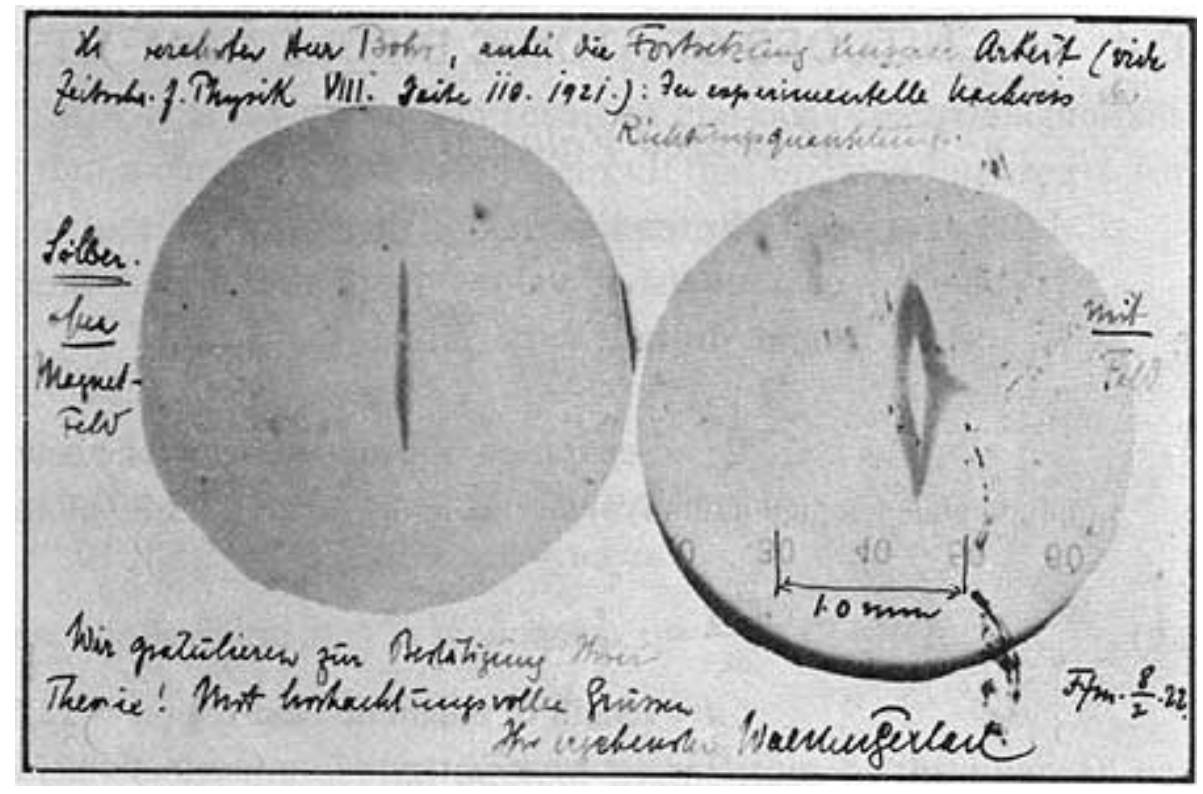

Figure 1.3. Original SGE results on a postcard to N. Bohr, dated Feb. 8, 1922. Image from Ref. 1, where the image was courtesy of AIP Emilio Segrè Visual Archives.

took almost a year to perform as the slits and magnet had to be aligned to within $10 \mu \mathrm{m}$, and the vacuum equipment would only function for a few hours at a time. According to legend $^{1}$, the resulting films of $\mathrm{Ag}$ on the recording plate was too thin to be visible, and was only noticed once it turned black after reacting with the sulfur in the cigars the two experimenters smoked while working in the lab (they soon switched to using a photographic development process to image the films). In 1922, they obtained their famous result: ${ }^{5}$ the beam was split into two by about $0.2 \mathrm{~mm}$. A postcard showing the resulting beam profiles with and without the magnet is shown in Figure 1.3.

The Stern-Gerlach experiment (SGE) results were interpreted as proving space quantization of the $\mathrm{Ag}$ atom angular momentum due to the orbiting electrons. The fact that the magnitude of the splitting corresponded to the predicted difference in magnetic moment of $2 \mu_{B}$ strengthened this interpretation. We now know that the Ag atom, with filled subshells (contributing zero net angular momentum) plus one 5 s electron, has $l=0$ in the ground state. The only possible value for the magnetic moment due to the orbiting motion of the electron is then 0 . Even if the $\mathrm{Ag}$ atoms were in an $l=1$ state, the possible values of $m_{L}$ would have been $+1,0$, and -1 , resulting in a beam split into three distinct beams, not two. 
While the initial interpretation of the results was correct in that it showed space quantization, it was incorrect about the source of the angular momentum. In fact, the splitting was due to an angular momentum (and magnetic moment) intrinsic to the electron itself, distinct from that due to its orbital dynamics, called spin. The beam splitting into exactly two parts clearly illustrates the binary nature of the electron spin. As one of the canonical experiments in quantum physics, the SGE is frequently cited for its fundamental significance as evidence of the electron spin as well as its simplicity and elegance. In fact, it is usually used as an introduction to quantum mechanics and a conceptual vehicle for assorted concepts in most quantum mechanics textbooks. In light of this, it is interesting to note that the SGE was originally interpreted without spin; spin was not "discovered" until 4 years later.

\subsubsection{Discovery - Uhlenbeck and Goudsmit}

Despite the seeming agreement between the Sommerfeld-Bohr model of angular momentum and the SGE, there were some inconsistencies with carefully scrutinized atomic emission/absorption spectra, including doublets, lines due to transitions forbidden in the Bohr model, and complex multiplet splittings with the application of a magnetic field (the "anomalous" Zeeman effect). A student of Paul Ehrenfest, Samuel Abraham Goudsmit spent much time working with Paschen learning the details of spectral analysis and the observed discrepancies. Intrigued by the forbidden lines, he recalled asking theoreticians about the observations, "But you know how theoreticians are ... then they say: 'Poor experiments.' " 6 He and a new student to the group, George Uhlenbeck, continued studying the spectrum, and worked with formula using half-integer quantum numbers to calculate the line locations (Lande's interval rule). When Goudsmit showed Uhlenbeck that he could give a simple interpretation of the recently published Pauli exclusion principle by introducing a new binary quantum number, Uhlenbeck recognized that it implied an extra degree of freedom intrinsic to the electron. ${ }^{\dagger}$ They interpreted the extra degree of freedom as a

\footnotetext{
${ }^{\dagger}$ In an amusing quote meant to capture the differences between the two physicists (differences which likely helped them make such an important breakthrough), Goudsmit describes, "In those days, all through the summer when I told Uhlenbeck about Landé and Heisenberg, for instance, or about Paschen, then he
} 
rotation which could take two values, giving the electron an intrinsic amount of angular momentum as well as a corresponding magnetic moment, separate from the angular momentum and magnetic moment due to its orbital motion. As the popular story goes ${ }^{6}$, after they gave their 1925 manuscript about the spinning electron to a very supportive Ehrenfest for publication in Naturwissenschaften, ${ }^{7}$ Uhlenbeck mentioned the idea of the spinning electron to Lorentz. Lorentz pointed out that it could not be true, as an electron spinning fast enough to give the necessary magnetic moment would require the surface of the "classical radius" of the electron to be moving faster than the speed of light. Uhlenbeck then asked for Ehrenfest to stop the submission, however he told them it was too late. Apparently, Kronig had made a similar proposal to Pauli a year earlier, but pushed it no further after the idea was ridiculed by Pauli.

Uhlenbeck and Goudsmit published a subsequent two page "Letter to the Editor" 8 in which they used the electron spin to explain the Zeeman splittings, the forbidden lines and the doublet fine structure of X-ray spectra including their width dependence on nuclear charge $\left(\propto Z^{4}\right)$ and given quantum numbers. They also managed to include mention that their spinning electron with two possible values allows the Pauli exclusion principle to function without assuming "an unmechanical 'duality' in the binding of electrons." 8 The article appeared in Nature followed by a seeming endorsement by Bohr himself: the electron spin was officially discovered.

Modern quantum mechanics now describes particles as having, in addition to their orbital angular momentum (given by equations 1.1 and 1.2), an intrinsic angular momentum, called spin, governed by similar quantization rules:

$$
\begin{gathered}
|\mathbf{S}|=\hbar \sqrt{s(s+1)} \text { and } \\
S_{z}=m_{s} \hbar .
\end{gathered}
$$

Similar to the rule for the z-component of angular momentum, $m_{s}$ can take on values from $-s$ to $s$ in integer sized steps. However, the rule for possible s values is different from those for $l$ values. The angular momentum commutation relations in quantum mechanics only

asked: 'Who is that?' He never heard of them, strange. And when he said: 'That means a fourth degree of freedom.' Then I asked him: 'What is a degree of freedom?' " 6 
restrict a general angular momentum to integer or half-integer quantum numbers. In the case of orbital angular momentum, the requirement for the particle's wavefunction to return to the same value after a full rotation through space eliminates the half-integer quantum numbers. Hence, the orbital quantum numbers, $l$ and $m_{L}$ must be integers. Spin, however, is not associated with the spatial part of a particle's wavefunction, and does not have this same restriction. The spin quantum numbers, $s$ and $m_{s}$, can be either integers or odd half-integers. The spin angular momentum is also slightly different from orbital angular momentum, in that the spin quantum number $s$ for a given particle is fixed. For example, particles called pions have $s=0$ and photons have $s=1$. Electrons (as well as proton and neutrons) have $s=1 / 2$ and are sometimes called spin-half particles. Thus, according to equation 1.9, electrons can have the component of their spin angular momentum along the z (or any other) axis equal to $+\hbar / 2$ or $-\hbar / 2$, spin-up or spin-down.

The sum of a particle's (or system of particles) angular momentum follows the same quantization rules as above. The letter $\mathrm{J}$ is usually used to signify the total angular momentum:

$$
\begin{aligned}
|\mathbf{J}| & =\hbar \sqrt{j(j+1)} \text { and } \\
J_{z} & =m_{j} \hbar
\end{aligned}
$$

The total momentum component along a spatial axis is the simple sum of the individual components. For a single electron, this means that the total angular momentum, $\mathbf{J}$, is given by the quantum numbers

$$
m_{j}=m_{L}+m_{s}
$$

from which it follows that

$$
|l-s| \leq j \leq l+s
$$

The total magnetic moment of an electron should then be given by equation 1.3 , but with the orbital angular momentum replaced by the total angular momentum as

$$
\boldsymbol{\mu}=\mu_{B}(\mathbf{J} / \hbar)=\frac{\mu_{B}}{\hbar}(\mathbf{L}+\mathbf{S}) .
$$

This expression is almost correct. In fact, the magnetic moment of the electron due to its spin is enhanced by almost exactly a factor two (the so-called electron spin gyromagnetic 
ratio, or ' $\mathrm{g}_{s}$-factor' is $\approx 2$ ), giving the correct expression for the total magnetic moment of an electron as

$$
\boldsymbol{\mu}=\mu_{B}(\mathbf{J} / \hbar)=\frac{\mu_{B}}{\hbar}(\mathbf{L}+2 \mathbf{S}) .
$$

As the spin of an electron is either $\pm \hbar / 2$, the intrinsic magnetic moment of an electron is given by

$$
\boldsymbol{\mu}=\frac{\mu_{B}}{\hbar} 2 \mathbf{S}= \pm \mu_{B}
$$

With electron spin in mind, we can now understand the SGE results. The filled subshells of the Ag atoms net zero orbital angular momentum. With each orbital filled with both a spin-up and -down electron, these shells net zero spin momentum as well. The final unpaired 5 s electron has $l=0$ and so the only contribution of angular momentum comes from the one electron spin. The two values of the spin component along the $\mathrm{z}$-axis is then the source of the two branches of the split beam. But what about the magnitude of the splitting? With $L=0$ and $S=1 / 2$, equation 1.15 gives the total magnetic moment of

the $\mathrm{Ag}$ atoms to be $\pm \mu_{B}$, in perfect agreement with Stern and Gerlach's results showing a splitting corresponding to a difference in magnetic moment of $2 \mu_{B}$. Although the results of the SGE were widely known, it is interesting to note that the discoverers of the spin of the electron made no mention of the SGE in relation to their discovery at the time.

It should also be noted that spin should not be interpreted as a classically rotating sphere. As Lorentz argued to Uhlenbeck, this was not possible. Spin is a purely quantum mechanical quality, following the quantum rules of angular momentum with quantum number $s=1 / 2$ with a magnetic moment of $\mu_{B}$. Both the spin and magnetic moment of the electron ( with $\mathrm{g}_{s} \approx 2$ ) are automatically contained in the relativistic Dirac description of electrons, but have no direct classical explanations.

\subsubsection{Spin-orbit coupling}

One of the many effects of the electron having an intrinsic spin angular momentum is the coupling between the spin and the electron's own orbital angular momentum. This is referred to as spin-orbit coupling and can be an important interaction in determining solid 
state electronic structures and can give rise to spin-polarization effects in photoemission experiments from non-magnetic systems. It is also the interaction at the heart of the most popular type of instrument for electron spin analysis.

The spin-orbit interaction falls naturally out of the Dirac equation without any extra assumptions. A more intuitive understanding can be gained by describing it as due to the interaction of the electron spin with an external electric field, E. At first glance, it is not obvious that the spin should interact with an electric field. However, if the electron is moving through $E$ in the lab reference frame with velocity $\mathbf{v}$, relativity requires that the electron experiences a magnetic field in its rest frame given by

$$
\mathbf{B}_{\mathrm{eff}}=-\frac{\mathbf{v}}{c} \times \mathbf{E}
$$

The electron's spin magnetic moment directly interacts with this magnetic field with the Hamiltonian

$$
H_{L S}=-\boldsymbol{\mu} \cdot \mathbf{B}_{\mathrm{eff}}
$$

Using equation 1.16 for $\boldsymbol{\mu}$ and substituting in equation 1.17 gives

$$
\begin{aligned}
H_{L S} & =\frac{2 \mu_{B}}{\hbar} \mathbf{S} \cdot\left[\frac{\mathbf{v}}{c} \times \mathbf{E}\right] \\
& =\frac{e}{m_{e}^{2} c^{2}} \mathbf{S} \cdot[\mathbf{p} \times \mathbf{E}]
\end{aligned}
$$

This expression is in fact not quite right, and results in atomic fine structure splittings a factor 2 too large. This was explained by Thomas in $1926^{9}$ as due to neglecting the fact that the electron's rest frame is not an inertial frame; when treated correctly, an extra factor $1 / 2$ is discovered, correcting the above expression to

$$
H_{L S}=\frac{e}{2 m_{e}^{2} c^{2}} \mathbf{S} \cdot[\mathbf{p} \times \mathbf{E}]
$$

If the electric field is that of a central potential, $V_{c}(r)$, as in the case of a hydrogen-like atom, the electric field can be expressed as

$$
\mathbf{E}=-\left(\frac{1}{e}\right) \nabla V_{c}(r)=-\left(\frac{1}{e}\right) \frac{\mathrm{d} V_{c}}{\mathrm{~d} r} \frac{\mathbf{x}}{r} .
$$


and inserted into equation 1.20 to give

$$
\begin{aligned}
H_{L S} & =\frac{1}{2 m_{e}^{2} c^{2}} \mathbf{S} \cdot\left[\frac{\mathrm{d} V_{c}}{\mathrm{~d} r} \frac{\mathbf{x}}{r} \times \mathbf{p}\right] \\
& =\frac{1}{2 m_{e}^{2} c^{2}} \frac{1}{r} \frac{\mathrm{d} V_{c}}{\mathrm{~d} r}(\mathbf{L} \cdot \mathbf{S}),
\end{aligned}
$$

where we have used the definition of orbital angular momentum, $\mathbf{L}=\mathbf{x} \times \mathbf{p}$. This last expression shows the origin of the name 'spin-orbit coupling' as the interaction depends on the term $\mathbf{L} \cdot \mathbf{S}$.

To proceed further, we define the total angular momentum of the electron to be $\mathbf{J}$ with

$$
\mathbf{J}=\mathbf{L}+\mathbf{S}
$$

Taking the dot product of equation 1.23 with itself gives

$$
\mathbf{J}^{2}=\mathbf{L}^{2}+\mathbf{S}^{2}+2(\mathbf{L} \cdot \mathbf{S})
$$

With this simple trick, equation 1.22 can be rewritten as

$$
H_{L S}=\frac{1}{2 m_{e}^{2} c^{2}} \frac{1}{r} \frac{\mathrm{d} V_{c}}{\mathrm{~d} r} \frac{1}{2}\left(\mathbf{J}^{2}-\mathbf{L}^{2}-\mathbf{S}^{2}\right)
$$

The spin-orbit Hamiltonian, $H_{L S}$, then splits atomic energy levels according to the total and orbital quantum numbers. Perturbation theory gives the first order energy shift of the levels with $j, l$, and the standard quantum number $n$ as

$$
\Delta_{n l j}=\frac{1}{2 m_{e}^{2} c^{2}}\left\langle\frac{1}{r} \frac{\mathrm{d} V_{c}}{\mathrm{~d} r}\right\rangle_{n l} \frac{\hbar^{2}}{2} \times\left\{\begin{array}{c}
l \\
-(l+1)
\end{array}\right\} \begin{aligned}
& j=l+1 / 2 \\
& j=l-1 / 2
\end{aligned} .
$$

This is Lande's interval rule.

It is important to remember that the spin-orbit interaction is a relativistic effect. As such, it is a large effect only when electron velocities are near the speed of light, clearly reflected in the $(\mathbf{v} / \mathrm{c})$ term in equation 1.17 . The spin-orbit interaction is thus important when dealing with free electrons with many keV of energy, or when dealing with electrons near high $\mathrm{Z}$ nuclei with large electric fields (large $d V_{c} / d r$ in equation 1.26). It should also be noted that the $\frac{1}{r} \frac{\mathrm{d} V_{c}}{\mathrm{~d} r}$ term in equation 1.22 and on show that in Coulomb potentials, the 
spin-orbit interaction falls off more rapidly with distance than the bare Coulomb interaction itself.

For hydrogenic atoms (i.e. ions with only one electron) where $V_{c}$ is of pure Coulomb form, we have

$$
\frac{\mathrm{d} V_{c}}{\mathrm{~d} r}=\frac{\mathrm{d}}{\mathrm{d} r}\left(\frac{Z e^{2}}{r}\right)=\frac{Z e^{2}}{r^{2}}
$$

and we then have

$$
\left\langle\frac{1}{r} \frac{\mathrm{d} V_{c}}{\mathrm{~d} r}\right\rangle_{n l}=\left(Z e^{2}\right)\left\langle\frac{1}{r^{3}}\right\rangle_{n l}=\frac{Z^{3}}{a_{0}^{3} n^{3} l(l+1 / 2)(l+1)}
$$

where $a_{0}$ is the Bohr radius. Equation 1.26 can then be expressed as

$$
\Delta_{n l j}=\frac{m c^{2}(Z \alpha)^{4}}{4 n^{3} l(l+1 / 2)(l+1)} \times\left\{\begin{array}{c}
l \\
-(l+1)
\end{array}\right\} \begin{aligned}
& j=l+1 / 2 \\
& j=l-1 / 2
\end{aligned}
$$

In this form (completely correct only if $V_{c}$ is a pure, unscreened Coulomb potential) we can see the $Z^{4}$ dependence mentioned by Uhlenbeck and Goudsmit in ref. 8 .

\subsubsection{Symmetry of spin}

The electron spin has other profound effects due to subtle, but fundamental, aspects of nature. One of these subtleties involves the fact that electrons, as well as other fundamental particles, are identical. An electron's only characteristics are its mass, charge, and spin, and since all electrons have the same values of these, electrons are said to be identical. This concept is perhaps best illustrated in Figure 1.4 which shows two electrons colliding. It is not possible to track each electron's path as that would disturb the system. Because the electrons are identical, a final measurement of the electrons' positions after the scattering event cannot distinguish, even in principle, whether the event took place as in panel (a) or (b). In fact they must both happen simultaneously as a quantum superposition.

For now let's consider just two electrons where electron 1 is in a state described by the ket $|\alpha\rangle$ and electron 2 is in a state described by the ket $|\beta\rangle$. The state for the system of two electrons could be well described by the ket $|\alpha\rangle|\beta\rangle$, but would be equally well described by $|\beta\rangle|\alpha\rangle$. These are two distinct state kets (and are in fact orthogonal assuming $\alpha \neq$ 
(a)

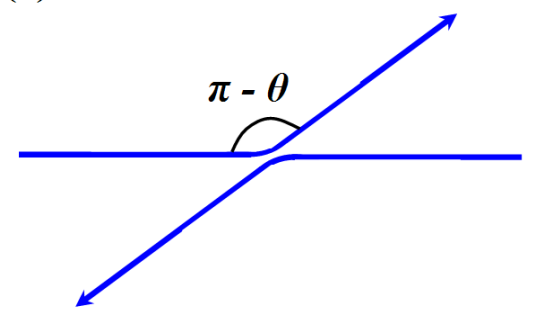

(b)

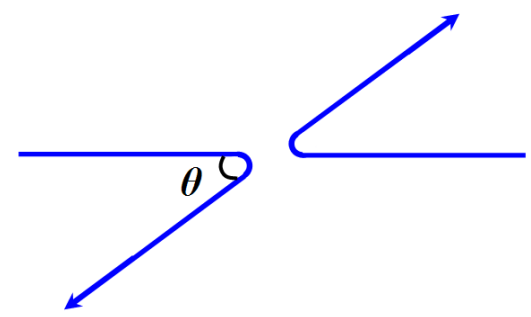

Figure 1.4. Indistinguishable scattering events of identical particles. It cannot be known, even in principle, which of (a) or (b) occurred.

$\beta$, and yet have the same exact set of eigenvalues for any possible measurement. This exchange degeneracy presents quantum mechanics with a tricky situation as a complete set of eigenvalues does not completely determine the state ket as it does for a single particle. The way nature apparently avoids this issue has fascinating consequences.

From the definition of what it means for a particle to be identical, it can be shown that any physical observable, including any physical Hamiltonian, must be invariant to the permutation of two of the identical particles, requiring the eigenstates of any observable to be simultaneous eigenstates of permutation. The permutation operator's, $P_{12}$, effect on a system can be written as

$$
P_{12}|\alpha\rangle|\beta\rangle=|\beta\rangle|\alpha\rangle
$$

or perhaps more clearly as

$$
P_{12}\left|\Psi\left(\mathbf{x}_{1}, \mathbf{x}_{2}\right)\right\rangle=\left|\Psi\left(\mathbf{x}_{1}, \mathbf{x}_{2}\right)\right\rangle .
$$

It should be clear that $P_{12}=P_{21}$, meaning its eigenvalues are \pm 1 . Two particle eigenstates of permutation are said to be symmetric with an eigenvalue of +1 and antisymmetric with an eigenvalue of -1 . For the two particle example above, the possible states are then the symmetric state

$$
\frac{1}{\sqrt{2}}(|\alpha\rangle|\beta\rangle+|\beta\rangle|\alpha\rangle)
$$

and the antisymmetric state

$$
\frac{1}{\sqrt{2}}(|\alpha\rangle|\beta\rangle-|\beta\rangle|\alpha\rangle) .
$$


Technically, with systems of more than two particles, a state can be constructed that is a symmetric eigenket of $P_{12}$ and and an antisymmetric eigenket of $P_{23}$, for example, and such a state is said to have mixed symmetry. Because the Hamiltonian must be invariant to permutation, a state's permutation symmetry is fixed for all time.

As far as we know, all systems of identical particles either completely symmetric or antisymmetric with respect to permutation - no mixed symmetry ever occurs. This is called the "Symmetrization Postulate" and is an empirical fact of nature, underivable from the fundamental postulates of quantum mechanics. ${ }^{\dagger}$ Bosons are particles which form systems that are completely symmetric and hence obey Bose-Einstein statistics. Fermions are particles which form systems that are completely antisymmetric and hence obey Fermi-Dirac statistics. A most amazing result is the connection between permutation symmetry and spin: integer spin particles are bosons and half-integer spin particles are fermions. This seemingly arbitrary rule is in fact derivable from relativistic quantum mechanics which proves that integer spin particles cannot be fermions and half-integer spin particles cannot be bosons. ${ }^{11}$ This connection is typically referred to as the "spin-statistics theorem" and is one of the most intriguing aspects of spin. Actually understanding the proof, however, is anything but elementary and attempts for more intuitive explanations have been revisited. ${ }^{12}$

Electrons, with spin $s=1 / 2$, are then fermions, and they must form states which are completely antisymmetric with respect to permutation. This has immediate far-reaching consequences as antisymmetric states cannot be formed if two particles are in the same state. For example, the required antisymmetric state of three electrons in $|\alpha\rangle,|\beta\rangle$, and $|\gamma\rangle$ is

$$
\left|\Psi_{\alpha \beta \gamma}\right\rangle=\frac{1}{\sqrt{6}}(|\alpha\rangle|\beta\rangle|\gamma\rangle-|\beta\rangle|\alpha\rangle|\gamma\rangle+|\gamma\rangle|\alpha\rangle|\beta\rangle-|\alpha\rangle|\gamma\rangle|\beta\rangle+|\beta\rangle|\gamma\rangle|\alpha\rangle-|\gamma\rangle|\beta\rangle|\alpha\rangle)
$$

Because $\left|\Psi_{\alpha \beta \gamma}\right\rangle=0$ if $|\alpha\rangle=|\beta\rangle$, it is clear that a system of many electrons, and other fermions, cannot exist with more than one electron in a given state with all equal quantum numbers (this result holds, of course, for system of arbitrary $N$ fermions). This is the cause

\footnotetext{
${ }^{\dagger}$ Many quantum physics textbooks suggest that this is derivable from quantum mechanics, but these treatments ignore the possibility of mixed symmetry states. An illuminating discussion is found in Ballentine's textbook. ${ }^{10}$
} 
of the Pauli exclusion principle, which needed Uhlenbeck and Goudsmit's fourth degree of freedom (section 1.2.2) to agree with experiment. The varying structure of the periodic table can be said to be due to the Pauli exclusion principle, giving reason to argue that the spin$1 / 2$ nature of the electron, along with the Symmetrization Postulate, is quite consequential indeed.

It is illuminating to investigate the example of a two-electron system. The antisymmetric requirement of a multi-electron system applies to the full wavefunction. Assuming the Hamiltonian of the system commutes with $\mathbf{S}_{\text {tot }}^{2}$, the energy eigenfunction can be split into a product of a spatially dependent wavefunction, and a spin function which is independent of spatial coordinates. The eigenfunction can be written as

$$
\psi=\phi\left(\mathbf{x}_{1}, \mathbf{x}_{2}\right) \chi\left(m_{s 1}, m_{s 2}\right)
$$

Each function must be either symmetric or antisymmetric with respect to particle exchange. The antisymmetric requirement of the full function requires that if $\phi\left(\mathbf{x}_{1}, \mathbf{x}_{2}\right)$ is symmetric, then $\chi$ must be antisymmetric, and vice versa.

In the following, spin states are written with arrows to signify the value of $m_{s}$ for each particle. For example, $|\uparrow \downarrow\rangle$ corresponds to the state where particle 1 has $m_{s}=1 / 2$ (spinup) and particle 2 has $m_{s}=-1 / 2$ (spin-down). The three possible symmetric forms of $\chi$ are

$$
\chi_{t}=\left\{\begin{array}{l}
|\uparrow \uparrow\rangle \\
\frac{1}{\sqrt{2}}(|\uparrow \downarrow\rangle+|\downarrow \uparrow\rangle) \\
|\downarrow \downarrow\rangle
\end{array}\right.
$$

and are called spin triplet states. The single possible antisymmetric form is

$$
\chi_{s}=\frac{1}{\sqrt{2}}(|\uparrow \downarrow\rangle-|\downarrow \uparrow\rangle)
$$

and is called a spin singlet state. With the total spin operator defined as $\mathbf{S}_{\text {tot }}=\mathbf{S}_{1}+\mathbf{S}_{2}$, the spin quantum number $s=1$ for the triplet states, with $m_{s}=m_{s 1}+m_{s 2}=1,0$ and 1, accordingly. For the singlet state, $s=0$ and of course $m_{s}=0$. Again, due to the antisymmetric requirement for the total wavefunction in equation 1.35, triplet spin states must have antisymmetric spatial wavefunctions, $\phi\left(\mathbf{x}_{1}, \mathbf{x}_{2}\right)$, and singlet spin states must have 
symmetric spatial wavefunctions. As we will see below, these consequences of electron spin and the Symmetrization Postulate allow spin dependence to sneak into systems where no explicit spin dependence is originally present.

\subsubsection{Exchange coupling}

Interactions and properties of many-electron systems which result from the requirement of antisymmetry in particle permutation are usually described by the term exchange. As we will see below, these exchange interactions can lead to important phenomena such as Hund's first rule and ferromagnetism. Throughout the discussion, it is important to remember that exchange coupling brings about effective spin dependent interactions even with Hamiltonians that contain no explicit spin dependence.

A ferromagnet is a material in which the atomic dipole moments become aligned parallel leading to a net spontaneous magnetization, even in the absence of an external applied magnetic field. Only Fe, Ni, Co, Gd, and Dy show elemental ferromagnetism. The interaction which aligns the atomic dipoles is often modeled as an effective internal magnetic field, historically referred to as a Weiss or molecular field. The thermal energy in a material will tend to randomize the dipole moments, and so if the temperature is raised high enough, eventually the thermal randomization will win out over the Weiss field and the material will become a paramagnet. This temperature is called the critical temperature, or $\mathrm{T}_{C}$. If each atom has a dipole moment of $1 \mu_{\mathrm{B}}$, then the above statement could be expressed as

$$
k_{\mathrm{B}} T_{\mathrm{C}} \approx \mu_{\mathrm{B}} H_{W}
$$

where $k_{B}$ is Boltzmann's constant and $H_{W}$ in the Weiss field. For a $\mathrm{T}_{C}$ of about $1000 \mathrm{~K}$ (close to the $\mathrm{T}_{C}$ of bulk Fe), this gives a Weiss field of about $10^{7}$ Gauss, which is a bit larger than the largest field produced in any laboratory! So the source of the Weiss field is no small effect.

It is tempting to think that direct dipolar interactions are behind it, where one atomic dipole interacts with the magnetic field of a neighboring dipole. We can investigate this possibility by writing the direct classical dipole interaction energy of dipoles $\mathbf{m}_{1}$ and $\mathbf{m}_{2}$ 
separated by the vector $\mathbf{r}$ as

$$
U_{d i p}=\frac{1}{r^{3}}\left[\mathbf{m}_{1} \cdot \mathbf{m}_{2}-3\left(\mathbf{m}_{1} \cdot \hat{\mathbf{r}}\right)\left(\mathbf{m}_{2} \cdot \hat{\mathbf{r}}\right)\right]
$$

Without being too precise, we can estimate that each dipole has a magnitude of about $\mu_{\mathrm{B}}$. Disregarding the angular dependence of the dot products, this gives us on the order of

$$
U_{d i p} \approx \frac{\mu_{\mathrm{B}}^{2}}{r^{3}} \approx \frac{1}{(137)^{2}}\left(\frac{a_{0}}{r}\right)^{3} R y
$$

where $a_{0}$ is the Bohr radius and 1 Ry is about $13.6 \mathrm{eV}$. With a dipole separation distance of about $2 \AA \sim 4 a_{0}$, this gives

$$
U_{d i p} \sim 10^{-5} \mathrm{eV}
$$

As only $1 \mathrm{~K} \sim 10^{-4} \mathrm{eV}$, thermal fluctuations will clearly outweigh any dipolar interactions well before the typical $\mathrm{T}_{C}$ of $1000 \mathrm{~K}$ used above. Looking at it in another way, the magnetic field of a $\mu_{\mathrm{B}}$ dipole at a distance of $2 \AA$ is only about $10^{3}$ Gauss, far smaller than the necessary Weiss field calculated above from equation 1.38. Therefore it is clear that direct dipole-dipole interactions are not the source of ferromagnetic ordering (although they play a key role in domain formation, well described in chapter 33 of ref. 13).

In 1928, Heisenberg showed the Weiss field originates from the exchange interaction. ${ }^{14}$ To illustrate the nature of the exchange interaction, it is useful to investigate examples of two-electron system. The simplest system is the case of two free non-interacting electrons, with Hamiltonian

$$
H=-\frac{\hbar^{2}}{2 m_{e}}\left(\nabla_{1}^{2}+\nabla_{2}^{2}\right)
$$

where the subscripts 1 and 2 refer to the two electrons. The eigenvalue equation $H \Psi\left(\mathbf{x}_{1}, \mathbf{x}_{2}\right)=E \Psi\left(\mathbf{x}_{1}, \mathbf{x}_{2}\right)$ is separable into a product of two single electron functions, and the solution can be written

$$
\Psi\left(\mathbf{x}_{1}, \mathbf{x}_{2}\right)=\psi_{a}\left(\mathbf{x}_{1}\right) \psi_{b}\left(\mathbf{x}_{2}\right)=e^{i \mathbf{k}_{a} \cdot \mathbf{x}_{1}} e^{i \mathbf{k}_{b} \cdot \mathbf{x}_{2}}
$$

Since the total state must be antisymmetric with respect to particle exchange (Symmetrization Postulate), the spatial part must be symmetrized/antisymmetrized versions 
of $\Psi\left(\mathbf{x}_{1}, \mathbf{x}_{2}\right)$ which can be written

$$
\begin{aligned}
\Psi_{ \pm}\left(\mathbf{x}_{1}, \mathbf{x}_{2}\right) & =\frac{1}{\sqrt{2}}\left[e^{i \mathbf{k}_{a} \cdot \mathbf{x}_{1}} e^{i \mathbf{k}_{b} \cdot \mathbf{x}_{2}} \pm e^{i \mathbf{k}_{a} \cdot \mathbf{x}_{2}} e^{i \mathbf{k}_{b} \cdot \mathbf{x}_{1}}\right] \\
& =\frac{1}{\sqrt{2}}\left(e^{i\left(\mathbf{k}_{a}+\mathbf{k}_{b}\right) \cdot \mathbf{R}}\right)\left[e^{i\left(\mathbf{k}_{a}-\mathbf{k}_{b}\right) \cdot \mathbf{r} / 2} \pm e^{-i\left(\mathbf{k}_{a}-\mathbf{k}_{b}\right)}\right]
\end{aligned}
$$

where $r$ is the separation of the two electrons $\mathbf{x}_{1}-\mathbf{x}_{2}$, and $\mathbf{R}$ is the center of mass $\left(\mathbf{x}_{1}+\right.$ $\left.(x)_{2}\right) / 2 . \Psi_{+}$is symmetric, and so must be matched with an antisymmetric spin function, the singlet state, $\chi_{s}$, from equation 1.37. $\Psi_{-}$is antisymmetric, and so must be matched with a symmetric spin function, one of the triplet states, $\chi_{t}$, from equation 1.36. The probability densities, $\left|\Psi_{ \pm}\left(\mathbf{x}_{1}, \mathbf{x}_{2}\right)\right|^{2}$, for the two choices become

$$
\begin{aligned}
& 2\left|\cos \left[\left(\mathbf{k}_{a}-\mathbf{k}_{b}\right) \cdot \mathbf{r} / 2\right]\right|^{2} \quad \text { for the singlet state, } \\
& 2\left|\sin \left[\left(\mathbf{k}_{a}-\mathbf{k}_{b}\right) \cdot \mathbf{r} / 2\right]\right|^{2} \quad \text { for the triplet states. }
\end{aligned}
$$

Somewhat surprisingly, this shows that despite $H$ being free of any interaction between the two electrons, their positions are in fact correlated, and on top of that, the correlation is spin dependent! The Pauli exclusion principle is again illustrated by the fact that the triplet states (which feature parallel alignment of the spins) have vanishing probability densities when $\mathbf{k}_{a}=\mathbf{k}_{b}$. Two electrons cannot occupy the same states with the same spin direction. Also, when $\mathbf{k}_{a} \neq \mathbf{k}_{b}$, we see that at $\mathbf{r}=0$, the singlet (triplet) state has maximum (vanishing) probability density. This suggests that the electrons in spin singlet states are somewhat "social" and find themselves spatially near each other, while those in triplet spin states are very "antisocial" and ensure they are never right next to each other. This exchange effect also holds when there are explicit electron-electron interactions and suggests that if there were a Coulomb repulsion between the two electrons, the triplet state would have a lower total energy. This concept is qualitatively at the root of Hund's first rule which prefers maximum parallel alignment of spins in an atomic subshell.

This approach can followed with slightly more complex two-electron problems such as the helium atom, hydrogen molecule, or similar systems. The Hamiltonian without direct electron-electron interaction is

$$
H_{0}=-\frac{\hbar^{2}}{2 m_{e}}\left(\nabla_{1}^{2}+\nabla_{2}^{2}\right)+V\left(q_{1}\right)+V\left(q_{2}\right)
$$


This Hamiltonian is separable, and so similar to equation 1.43, possible wavefunctions include (with clear shorthand notation)

$$
\Psi(1,2)=\phi_{a}(1) \phi_{b}(2)
$$

where $\phi_{a}(1)$ is the solution to

$$
\left[-\frac{\hbar^{2}}{2 m_{e}} \nabla_{1}^{2}+V\left(q_{1}\right)\right] \phi=E_{a} \phi
$$

and $\phi_{b}(2)$ is the solution to the equivalent solution for electron 2 with energy $E_{b}$. Again, just as above, the correct wavefunctions must be symmetrized/antisymmetrized and matched with the correct spin singlet/triplet states. The two total wavefunctions are then:

$$
\begin{array}{llr}
\Psi_{s} & =\frac{1}{\sqrt{2}}\left[\phi_{a}(1) \phi_{b}(2)+\phi_{a}(2) \phi_{b}(1)\right] \chi_{s} & \text { singlet } \\
\Psi_{t}=\frac{1}{\sqrt{2}}\left[\phi_{a}(1) \phi_{b}(2)-\phi_{a}(2) \phi_{b}(1)\right] \chi_{t} & \text { triplet. }
\end{array}
$$

It should be clear from the example of the free electrons that these wavefunctions will have position correlation. However, there is no energy difference between them yet; $E=$ $\int \Psi_{s}^{*} H \Psi_{s} \mathrm{~d} \mathbf{x}_{1} \mathrm{~d} \mathbf{x}_{2}=\int \Psi_{t}^{*} H \Psi_{t} \mathrm{~d} \mathbf{x}_{1} \mathrm{~d} \mathbf{x}_{2}$. Now let's introduce electron-electron interaction through a perturbation, $H_{12}$. This can be as simple as Coulomb repulsion between the two electrons as $H_{12}=\frac{e^{2}}{r_{12}}$ or something more complex, perhaps involving the additional interaction between electron 1 and nucleus 2 (and vice versa) and the interaction between the two nuclei in the case of the hydrogen molecule. The additional energies introduced by $H_{12}$ (assuming $\Psi_{\text {I\&II }}$ remain good zero-order wavefunctions) can be calculated by $E_{s, t}=$ $\int \Psi_{s, t}^{*} H_{12} \Psi_{s, t}$ giving:

$$
\begin{aligned}
& E_{s}=\left(K_{12}+J_{12}\right) \\
& E_{t}=\left(K_{12}-J_{12}\right)
\end{aligned}
$$

with

$$
K_{12}=\int \phi_{a}^{*}(1) \phi_{b}^{*}(2) H_{12} \phi_{a}(1) \phi_{b}(2) \mathrm{d} \mathbf{x}_{1} \mathrm{~d} \mathbf{x}_{2}
$$

and

$$
J_{12}=\int \phi_{a}^{*}(1) \phi_{b}^{*}(2) H_{12} \phi_{a}(2) \phi_{b}(1) \mathrm{d} \mathbf{x}_{1} \mathrm{~d} \mathbf{x}_{2}
$$


$K_{12}$ is simply the average interaction energy that would be present without antisymmetrization of the full wavefunctions. $J_{12}$, however, is referred to as the exchange integral, and results directly from the Symmetrization Postulate and the odd half-integer spin of the electrons.

Equations 1.53 and 1.54 can be written as one equation by inspecting the total spin operator in a similar manner to equations 1.23 and 1.24 :

$$
\begin{aligned}
\mathbf{S} & =\mathbf{S}_{1}+\mathbf{S}_{2} \\
\mathbf{S}^{2} & =\mathbf{S}_{1}^{2}+\mathbf{S}_{2}^{2}+2 \mathbf{S}_{1} \cdot \mathbf{S}_{2} .
\end{aligned}
$$

From equation 1.8 we have $\mathbf{S}_{1}^{2}=\mathbf{S}_{2}^{2}=3 / 4$, and from these equations and the previous section we know $\left(\mathbf{S}^{2}\right)_{s}=0$ and $\left(\mathbf{S}^{2}\right)_{t}=2$. This allows equation 1.58 to be written for the singlet and triplet states as

$$
\begin{aligned}
& {\left[2 \mathbf{S}_{1} \cdot \mathbf{S}_{2}\right]_{s}=-\frac{3}{2}} \\
& {\left[2 \mathbf{S}_{1} \cdot \mathbf{S}_{2}\right]_{t}=+\frac{1}{2}}
\end{aligned}
$$

Using these expressions, equations 1.53 and 1.54 can be simultaneously written as

$$
E=K_{12}-\frac{1}{2} J_{12}-2 J_{12} \mathbf{S}_{1} \cdot \mathbf{S}_{2}
$$

This is an amazing result! Clear spin dependence is found, even though the Hamiltonian has no explicit spin dependence. The exchange coupling, $J$, is not a new interaction, but a direct result of the Coulomb interaction between the electrons and the required asymmetry of the electronic states with respect to electron exchange. It is interesting that the spin coupling in equation 1.61 does not depend on their exact orientation with respect to any axis, but only on their relative orientation to each other, and is a consequence of the original Hamiltonian's spin-independence. ${ }^{13}$

The approach to obtaining equation 1.61 can be similarly followed for many-electron systems such as in the solid state. Often times, the spin dependence due to the exchange interaction of such systems can be modeled with the Heisenberg spin Hamiltonian

$$
H_{\text {spin }}=-2 \sum_{i, j} J_{i j} \mathbf{S}_{i} \cdot \mathbf{S}_{j}
$$


where the sum is over an appropriate set of nearest neighbors, $j$, of site $i$, as well as over all $i$ in the crystal. If all the $J_{i j}$ are positive, parallel alignment of all spins becomes energetically favorable, and hence the exchange interaction is qualitatively the source of the Weiss field and ferromagnetism. Note that although it appears simple enough, solving the Heisenberg Hamiltonian for real systems is an enormous challenge, and can only be done with additional approximations at extreme temperatures far from $\mathrm{T}_{C}$. It is interesting enough to again stress that magnetic effects such as ferromagnetism are not the result of direct magnetic interactions, but are actually the result of electrostatic interactions. Much of this and the previous section (sections 1.2.5 and 1.2.4) follow the arguments and mathematics of refs. $15,13,14,10$, which give a more in-depth review of the topic. What is presented here is sufficient for the scope of this thesis.

\subsection{Probing spin in solids}

Various effects and interactions dependent on the electron spin, some of which were outlined in section 1.2, can play key roles in the electronic structure of solids. In many cases, the exact role is far from understood. Even in what appears to be simple cases, theory is not yet complete. For example, although low temperature itinerant $3 \mathrm{~d}$ ferromagnetism in the transition metals is fairly well understood, an accurate description around $\mathrm{T}_{C}$ is still missing. ${ }^{16}$ The more complex systems which currently get an enormous amount of attention have even larger gaps in understanding of the role of spin. The high transition temperature (HTC) superconductors are based on antiferromagnetic parent compounds. The exact role of remaining antiferromagnetic interactions and fluctuations in the formation of Cooper pairs is fiercely debated. The colossal magnetoresistive (CMR) manganites feature simultaneous insulating-to-metallic and paramagnetic-to-ferromagnetic transitions, and feature a wide array of magnetic structures with small amounts of chemical doping. An understanding of this class of materials is incomplete without a full picture of the spin dependence of their electronic structures. The whole exploding field of advanced electronic 
devices utilizing the electron spin (spintronics ${ }^{17,18}$ ) explicitly relies on understanding and manipulating the spin interactions in a wide variety of materials.

Large amounts of progress in our understanding of these and other systems, including new systems possibly discovered in the near future, can be made by probing their electronic structure in a spin-sensitive manner. Spin and angle resolved photoemission spectroscopy (spin-ARPES) is the most direct and complete technique for achieving this. Performed on ferromagnets, spin-ARPES can directly reveal the exchange split minority and majority bands, and probe interesting aspects such as the temperature dependence of the spin distribution as $\mathrm{T}_{C}$ is approached and crossed. Applied to spintronics materials and devices, spin-ARPES can directly illuminate the spin character of the electronic structure for comparison with the desired or predicted behaviors. Spin-ARPES performed with circularly polarized light on nonmagnetic metallic and semiconducting surfaces can generate spin-polarized photoelectrons due to spin-orbit coupling, providing additional information (including direct observation of the symmetry of the probed states) not accessible without spin-resolution. There have even been examples of insights being obtained by performing spin-resolved photoemission on the HTC cuprates, ${ }^{19,20,21}$ despite their antiferromagnetic background.

Although spin-ARPES is an extremely powerful way to experimentally probe these systems, the technique has been somewhat ignored in comparison to "regular" spin-integrated ARPES. This is not to say that great spin-ARPES work has not been done. Fantastic reviews of the field are given in refs. 16,22,23 with more recent results reviewed in ref. 24. However, while ARPES has grown remarkably during the past few decades in both popularity and impact, spin resolved photoemission has not yet been able to achieve the same scale of growth in widespread acclaim. Much of the success of ARPES is due to giant leaps in instrumental capabilities and efficiencies, allowing data to be taken with angular and energy resolutions at and below the scale needed to observe the most interesting physics in today's correlated electron research. Instrumentation for spin-resolved experiments have not achieved the same gains in efficiency, forcing the technique to be performed with significantly compromised resolutions, and hence greatly slowing the advancement of 
spin-ARPES. As we will see, the main difficulty lies in the actual detection of spin polarization; measuring whether an electron is spin-up or -down is not at all as straightforward as it naively seems. For instance, current state-of-the-art spin polarimeters introduce an efficiency reduction of $\sim 10^{-4}$ to determine the spin polarization of a beam compared to spin-integrated electron counting. With the additional loss of parallel energy and angle detection of current ARPES instruments, spin-ARPES is a very inefficient process.

Motivated by the tremendous need for progress in spin-resolved instrumentation, I have dedicated my Ph.D. work to the design and development of a novel instrument: the spinTOF spectrometer. This spectrometer aims to revolutionize spin-ARPES by achieving a breakthrough in possible resolutions through a 1000 times improvement in overall efficiency, thereby expanding the frontiers of the field. This is accomplished by attacking the above problems in two ways. First, a novel spin-polarimeter has been developed which offers up to 100 times improved efficiency over the current state-of-the-art polarimeters. Second, parallel energy detection is re-introduced to the spin-resolved experiment with the development of a time-of-flight (TOF) based energy analyzer to work in concert with the spin-polarimeter, introducing an extra factor of 10 gain in overall efficiency.

The thesis is organized as follows. Chapter 2 summarizes the experimental and theoretical details of the ARPES technique and describes the development of an advanced in-lab ARPES setup. Chapter 3 discusses the extension of ARPES with spin-resolution, reviews details of its experimental difficulties, and introduces how the spin-TOF will attack these difficulties. Chapter 4 covers the development of the TOF electrostatic lens system and Chapter 5 covers the development of the new spin polarimeter, including the basics of spin-polarimetry and the physical principles of current instruments. Chapter 6 summarizes the complete instrument and initial accomplishments. Chapter 7 concludes the thesis with a summary of the work to come as well as a description of some of the exciting future prospects for spin-ARPES with the spin-TOF. 


\section{Chapter 2}

\section{Photoemission Spectroscopy}

$I$ realized that electron spectroscopy for atoms and solids could never become competitive with X-ray emission or absorption spectroscopy unless I was able to achieve such a high resolution that really well-defined electron lines were obtained with linewidths equal to or close to the inherent atomic levels themselves.

— Kai Siegbahn 25

Photoemission spectroscopy is a broad class of experimental techniques which can be most concisely described as "photon-in, electron-out" experiments. A sample of interest is illuminated by a source of photons, and the intensity of the resulting photoejected free electrons is measured. This intensity can be a function of many parameters including the emitted electron's kinetic energy, direction of emission, and spin polarization, as well as photon energy and polarization. These dependencies result from details of the sample's electronic structure, thus offering a way to directly probe it. Different types of photoemission spectroscopy are defined by which of these dependencies are resolved in the measurement. For example, general photoemission spectroscopy (PES) only requires measuring the intensity as a function of photoelectron kinetic energy and typically integrates the intensity from a wide emission cone. Angle resolved photoemission spectroscopy (ARPES) measures the intensity as a function of photoelectron kinetic energy and emission direction. Spin and an-

gle resolved photoemission spectroscopy (spin-ARPES) additionally measures the intensity as a function of spin polarization. Typically all of these techniques utilize a photon beam 
of well-defined energy and polarization, and measuring the photon energy and polarization dependencies can be an additional component to each technique. Because spin-ARPES, performed with a light source offering full photon energy and polarization control, resolves and controls so many of the possible photointensity dependencies, it is sometimes referred to as the "complete photoemission experiment".

Due to the enormous amount of success and popularity that ARPES has achieved, there are many textbooks ${ }^{26,27}$ and review articles ${ }^{28,29,30}$ covering the full complexity of the techniques with both keen insight and sometimes an intimidating amount of detail. Because this thesis revolves around the development of an instrument to expand the boundaries of ARPES, basic details of the technique will be reviewed to highlight the instrumental improvements which made the power of today's ARPES possible.

\subsection{The photoelectric effect}

The physical basis of PES is of course the celebrated photoelectric effect, and so PES shares an important historical part of the development of quantum physics. A vivid history of the photoelectric effect and its development into modern PES can be found in ref. 31. The first written observation of the photoelectric effect was provided by Heinrich Hertz in $1887^{32}$ when he described his landmark experiments diagrammed in Figure 2.1. The experiment consisted of two spark-gaps driven by large inductor coils. A large current would flow through the coils, so that when a switch suddenly opened the circuit, the energy in the inductors could drive a spark across the gaps if the gaps were small enough. Hertz found that the occurrence of a spark at the primary gap would increase the chance of a spark at the second gap, which could be precisely controlled by a micrometer. By placing various barriers in the line of sight between the two spark-gaps, he found that this influence could be removed, and subsequently concluded that UV light emitted from the primary spark helped initiate the secondary spark. Although Hertz never returned to photoelectric experiments, he "sparked" a furious investigation of the effect by others.

Among the many scientists who made valuable insights into the phenomena, one of the 


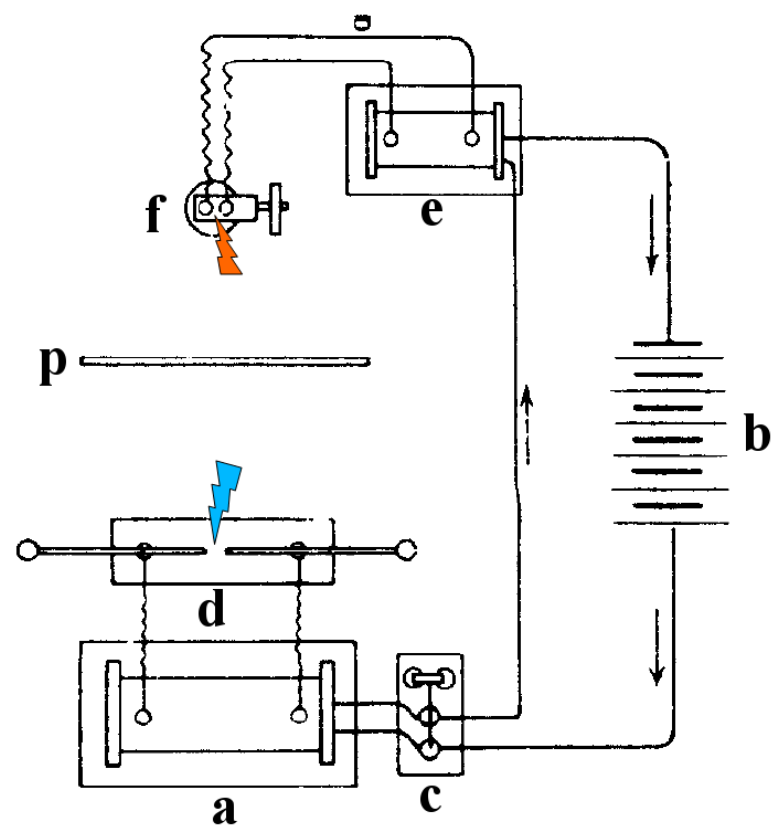

Figure 2.1. Schematic of Hertz's famous experiment demonstrating the photoelectric effect. Large inductors (a,e) powered by a battery (b) drive spark gaps (d,f). When switch (c) is open, primary spark at (d) was found to influence the possibility of spark at (f). Influence of primary spark could be shielded with a metal plate (p). Figure adapted from ref. 31.

most influential contributions was performed by Philipp Lenard, who began his career as an assistant to Hertz. Lenard began to investigate the velocity distribution of the photoelectrons, although at the time they were not understood to be electrons, but were referred to as cathode rays. He wondered if adsorbed gases on the cathode had an influence on his measurements, and he began doing his photoemission experiments in vacuum tubes, such as shown in Figure 2.2(a). With such an apparatus, he performed many experiments on the effect of external electric and magnetic fields on the cathode rays, and used retarding potentials at the anodes to study their velocity distributions. He came to three key conclusions in 1902 which had no immediate explanation. ${ }^{33}$ First he noted that the number of cathode rays depended on the light intensity. Second, he found that cathode ray velocity did not, however, depend on the light intensity. Finally, he found that the velocity was dependent on the light frequency. Lenard later received the 1905 Nobel prize for his work with cathode rays, which he described as "negatively charged quanta of electricity". It is interesting to 
(a)

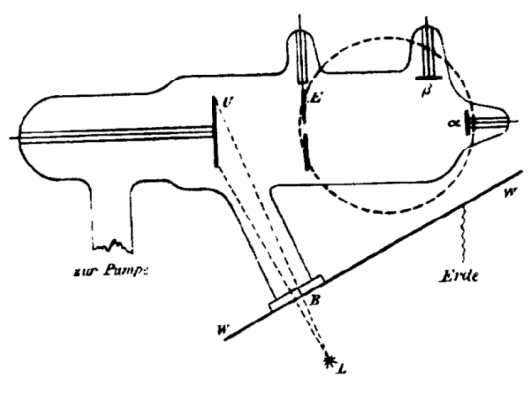

(b)

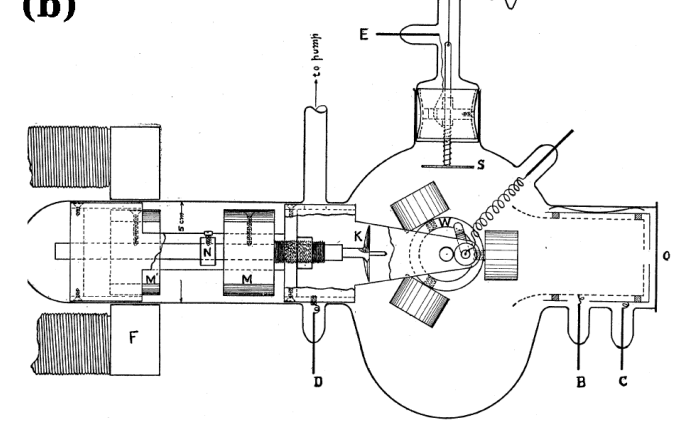

Figure 2.2. (a) Vacuum tube for Lenard's photoemission experiments. UV light from (L) excites the cathode $(\mathrm{U})$. Cathode rays enter experiment region passing through metal foil (E) and are measured at anodes $(\alpha, \beta)$. Figure taken from ref. 31. (b) Millikan's vacuum instrument used to verify Einstein's photoelectric effect equation. Na, Li, an K samples rotated around on central wheel $(\mathrm{w})$. Electromagnet $(\mathrm{F})$ could couple to in-situ magnet M' and $\mathrm{M}$ to manipulate knife blade $(\mathrm{K})$ to scrape a fresh surface of a sample. Fresh surface could then be rotated towards light input $(\mathrm{O})$ and double Faraday-cup detector controlled by electrodes (B) and (C). Plate $\mathrm{S}$ was used to accurately determine work function difference between sample face and Faraday-cup. Original text notes that fresh shavings of the samples would fall to the bottom of the central chamber and aid in removing residual oxygen from the chamber - a getter pump! Figure taken from ref. 34.

note that Lenard's work led him to conclude that cathode rays were not material particles with mass, but rather were aether waves.

Lenard's conclusions about the photoelectric effect (plus the observation that emission begins immediately at the start of irradiation with no delay) became understood to be incompatible within the classical description of light based on Maxwell's equation where the energy of light is proportional to its intensity (not frequency) and is delivered to the cathode continuously and evenly distributed in space. Lenard's work spurred Einstein's famous 1905 paper ${ }^{35}$ which explained these observations by quantizing light into distinct particles (photons) with discreet amounts of energy dependent on the light's frequency. His picture explained the photoelectric effect with the famous equation which we now write as

$$
E_{\max }=h \nu-\Phi
$$

where $E_{\max }$ is the maximum energy of the emitted electrons, $h$ is Planck's constant, and $\nu$ is the irradiating light frequency. $\Phi$ is called the work function of the cathode material and 
is the minimum amount of energy an electron must gain to leave the sample ( $\Phi$ is generally between 4 to $5 \mathrm{eV}$ for most materials). This paper (only one of his "miracle" four published that year) later earned Einstein the Nobel in 1921, as well as helped spur the quantum revolution.

Although Einstein's equation is readily accepted by students in introductory physics classes today, it was not the case at the time. Many worked extensively with various experimental setups in attempts to verify or disprove the theory. Experiments tended to suggest the theory was correct, but failed to rigorously prove the linear relationship between $E_{\max }$ and $\nu$ with a slope of $h$, mostly due to an experimental inability to reliably measure $E_{\max }{ }^{31}$. Robert Millikan proved up to the task, and built a custom vacuum chamber ${ }^{34}$ he called "a true machine shop in vacuo" to investigate Einstein's description of the photoelectric effect. The resulting instrument is shown in Figure 2.2(b). The figure, well described in the original text, has a remarkable similarity to many of the techniques we currently use for manipulation and coupling various motions into the vacuum system. In fact it is difficult, while reading it, to remember that it was written in 1916 ! With it, he was able to prepare very clean alkali surfaces in-situ and obtained clear high energy cutoffs in the photoelectron's energy distribution using a retarding-potential Faraday-cup detector. He ascribes having a light source with a well defined energy (no stray light of higher energy) being key to measuring the well defined cutoffs. ${ }^{34}$ With these high energy cutoffs defining $E_{\max }$ at varying light frequencies, Millikan found a precisely linear relation and obtained an amazingly accurate measurement of $h$ in the process. ${ }^{34}$ These great achievements were clearly directly related to the jump in instrumental expertise that Millikan introduced. Although he was very disbelieving of the photon concept, his work proving Einstein's equation (along with his determination of the electron's $e / m$ ratio in his oil-drop experiment) was awarded the 1923 Nobel prize. As a further aside to the historical development of photoemission instrumentation, Figure 2.3 shows two prominent scientists in the early field (Elster and Geitel), illustrating that early instrumentation allowed photoemission setups to be truly "table-top" experiments! 


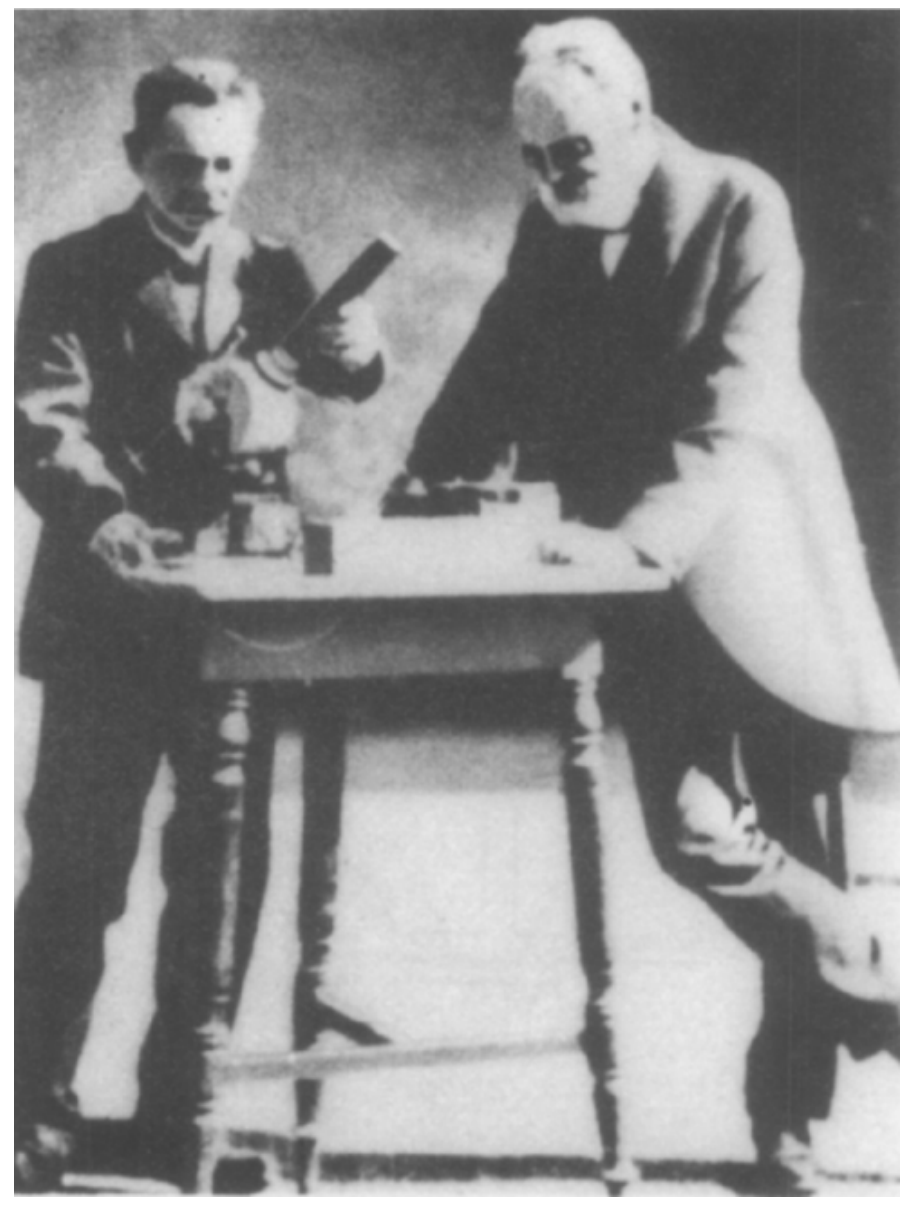

Figure 2.3. Julius Elster and Hans Geitel hard at work on their photoemission experiments. Figure from ref. 31. 


\subsection{Energy resolution}

The decades after the explanation of the photoelectric effect continued to see a huge amount of effort, both experimental and theoretical, aimed at understanding the photoelectric effect more thoroughly. Instrumental improvements in magnetic and electrostatic electron energy analyzers, various semi-monochromatic light sources, and improving vacuum pressures aided in the precision that photoelectron energies could be measured. Experimental effort was matched by the rapid progression in the theoretical understanding of atomic, molecular, and solid state structure with quantum theory. These advances allowed photoelectric experiments to evolve into a technique for studying the electronics of these structures.

Due to conservation of energy in the photoemission process, it was realized that measuring the kinetic energy of a photoemitted electron in vacuum allows the calculation of its binding energy in the solid if a well-monochromatized light source is used. This is expressed as

$$
E_{K}=h \nu-\Phi-\left|E_{B}\right|
$$

where $E_{K}$ is the kinetic energy of the photoelectron in vacuum, measured with respect to the vacuum level, $\Phi$ is the work function, $h \nu$ is the photon energy, and $E_{B}$ is the binding energy (a negative value measured with respect to the Fermi level, $\mathrm{E}_{F}$ ). If electrons emitted at all angles are collected together (an angle-integrated experiment), or equivalently a polycrystalline sample is used, the distribution of photoelectrons in kinetic energy can then effectively map the density of states (DOS) of the sample's electronic structure. This idea is shown schematically in Figure 2.4. A sample has a certain DOS $(N(E)$ in the figure) which includes the so-called "core levels" of distinct inner-shell electron energy states, as well as a valence band at $\mathrm{E}_{F}$ dictated by the energy bands formed by the delocalized valence electrons. An incident photon can promote an electron in this DOS upwards in the energy diagram by an amount $\hbar \omega$. Electrons promoted above the sample's vacuum level can physically escape the sample and can be detected by an energy resolving electron 


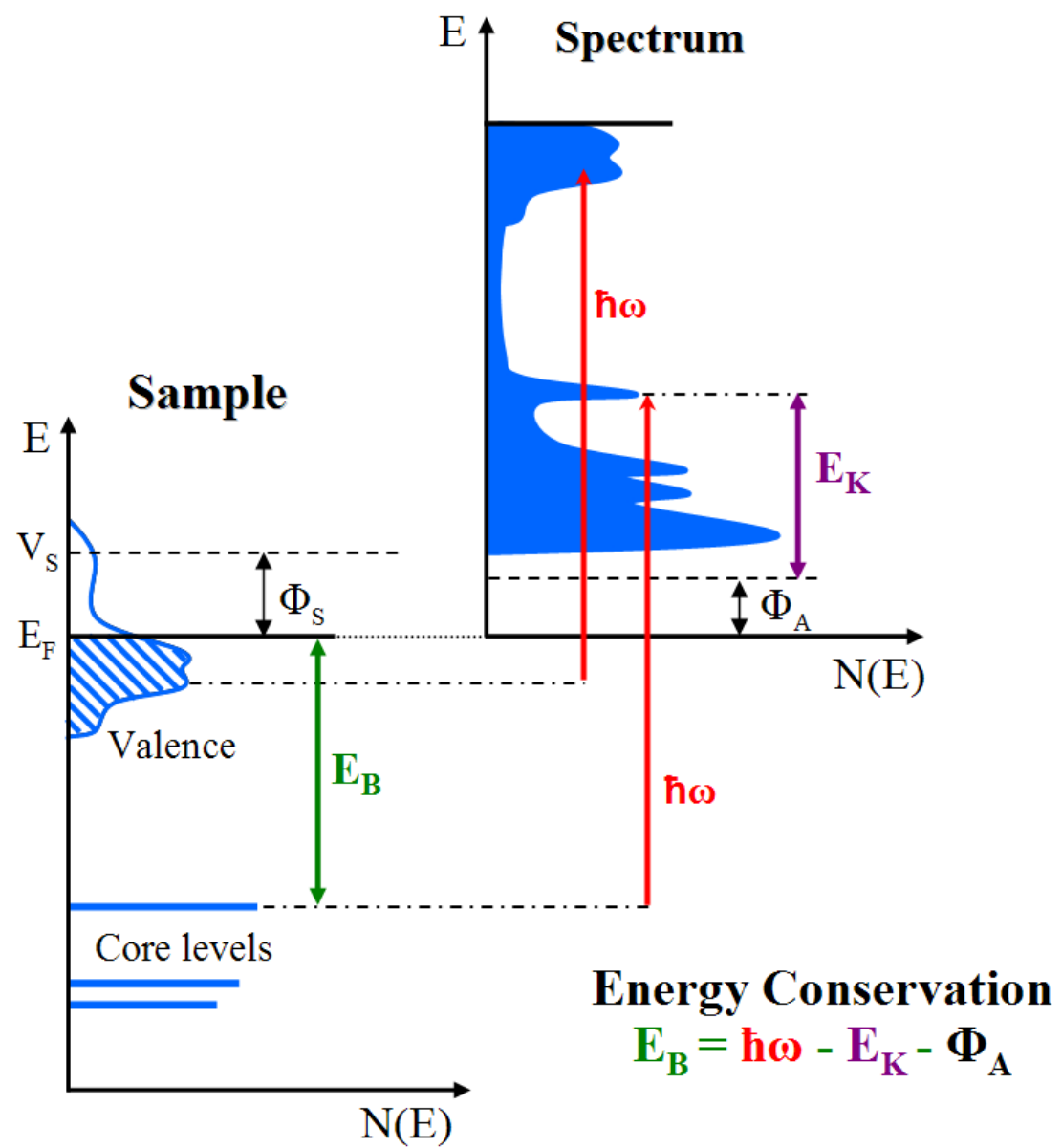

Figure 2.4. $\mathrm{N}(\mathrm{E})$ energy level diagram of a PES experiment. The $\mathrm{E}_{F}$ of the sample and analyzer are equal when electrically connected. $\Phi_{S(A)}$ and $V_{S(A)}$ are the work function and vacuum level of the sample (analyzer), respectively.

analyzer. Because of equation 2.2, the $N(E)$ inside the sample can be measured outside of the sample.

Although it is a relatively simple diagram, Figure 2.4 contains a number of interesting details. First, the electron occupation of the available DOS $\rightarrow 0$ following Fermi-Dirac statistics, and this will show up as a sharp cutoff in the measured spectrum. In other words, photoemission does not occur from states which are not actually occupied, meaning photoemission spectroscopy cannot readily access information about the electronic structure above $\mathrm{E}_{F}$. Experimentally this cutoff, referred to as the Fermi edge, provides a good 
reference to set the energy scale to. The width of the edge can also be used as a measure of the total energy resolution achieved in the experiment.

Second, the photoexcited electrons tend to have a short mean free path while still in the sample, and can suffer inelastic collisions before leaving through the sample surface. This can happen multiple times, with a random amount of energy lost each time, before an electron escapes into vacuum to be measured. Each collision can generate other excited electrons in the process, which themselves may suffer inelastic collisions before exiting the sample. This effect creates a cascade of "secondary" electrons, building in intensity towards lower kinetic energy in the spectrum. This indeed occurs, and causes the exponentially increasing secondary background in the measured spectrum as shown in the diagram. Note that a high enough photon energy is used so that the parts of the spectrum of interest are not clouded over by this background (although a common data analysis procedure can also be used to remove the "Shirley Background" from angle-integrated experiments ${ }^{36,37}$ ). In particular, the valence band which is of most interest to many in the solid-state community, is relatively free of this background as there are few electrons with higher energy.

Finally, the kinetic energy is measured with respect to some sort of analyzer, and so it is the work function of the analyzer, $\Phi_{A}$, which appears in the correct application of equation 2.2 , not the sample work function $\Phi_{S}$. The Fermi levels are forced to match when the sample and analyzer are electrically connected, and so the vacuum levels need not be the same. However, it is $\Phi_{S}$, which determines the "low-energy cutoff" where electrons can no longer escape the sample, coinciding with the vacuum level of the sample, $V_{S}$. Because $\Phi_{S}>\Phi_{A}$ in the particular instance of this diagram, the analyzer can directly measure this low-energy cutoff at a finite kinetic energy with respect to $V_{A}$. Note that the final "peak" in the measured spectrum is simply the low energy cutoff of secondary electrons.

Useful information can only be extracted from the PES experiment depicted in Figure 2.4 if it is done with a total energy resolution which allows observing the critical features, and it must be done with a high enough count rate such that the data can be recorded in a reasonable amount of time with good signal-to-noise ratios. This is where the work of Kai Siegbahn played an enormous role. Siegbahn began work in nuclear spectroscopy and 

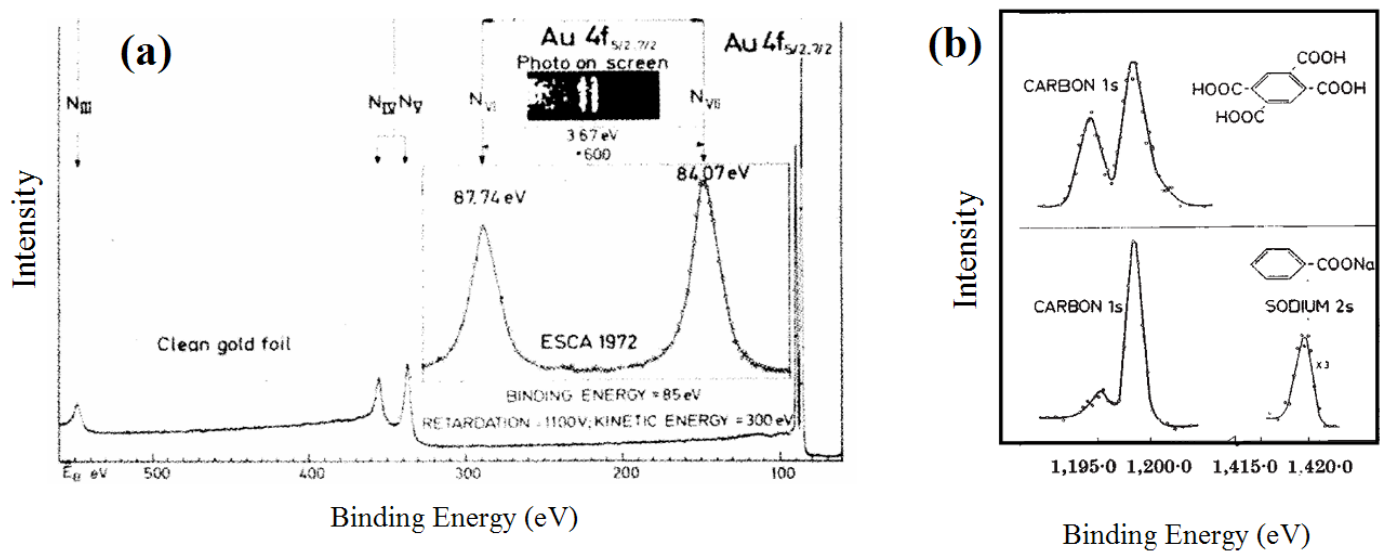

Figure 2.5. Examples of Siegbahn's revolutionary XPS work. (a) Au spectrum taken in Upsalla in 1972 with monochromatized AlK $\alpha$ excitation. Figure taken from ref. 25. (b) Benzenetetracarboxylic acid (upper) and sodium benzoate (lower) spectra showing well resolved chemical shifts of the $\mathrm{C}$ 1s peaks. Figure adapted from ref. 38 .

performed experiments in $\beta$-decay. In the process of his work, he needed to develop a high resolution, high efficiency analyzer, and ended up with a so-called "double-focusing" magnetic analyzer. His experiments had to suffer lengthy delays waiting for radioactive samples supplied by an unreliable synchrotron, and so began experimenting with simulating $\beta$-decay by photoemitting from lead foil with an X-ray tube. ${ }^{25}$ This aroused his interest in trying to measure the foil's binding energies with as high an energy resolution as possible. ${ }^{25}$ Similar work at the time was done with fairly poor resolution, such that the atomic core-levels were smeared out so much that they were not recognizable as individual atomic levels. Siegbahn set out to improve the energy resolution such that X-ray photoemission spectroscopy (XPS - PES with an X-ray light source) could give resolution of the atomic levels comparable to X-ray emission and absorption experiments. ${ }^{25}$

His adaptation of his successful $\beta$-decay analyzer led to an iron-free double-focus magnetic analyzer with a $30 \mathrm{~cm}$ radius that worked for the lower kinetic energies of XPS performed in the $5 \mathrm{keV}$ range. The analyzer achieved a resolving power $\left(\frac{\Delta E}{E}\right)$ of better than $10^{-4}$ with a count rate of about 1000 electrons per minute. ${ }^{25}$ With this instrument, the first high-resolution XPS spectra were recorded, resolving sharp atomic core levels of $\mathrm{Cu} .{ }^{39}$ Figure 2.5(a) shows an XPS spectrum taken by Siegbahn on Au, illustrating the fully resolved 
spin-orbit split 4f 5/2 and 7/2 core levels. Remarkably, Siegbahn's work was also able to resolve the core-levels well enough to observe their shifts in binding energy depending on the chemical environment of of the originating atom. ${ }^{40}$ These so-called "chemical shifts" are are nicely illustrated in Figure 2.5(b), one example of the many works to be based on this technique. The upper panel shows the $\mathrm{C}$ 1s peak split into two peaks corresponding to carbon atoms with the different bonding geometries of the carboxylic acid molecule being measured. Note that the peak ratios match the numerical ratios of the different carbon configurations in the molecule. This same concept is illustrated again in the lower panel as well for sodium benzoate. Siegbahn's introduction of vastly improved electron energy analyzers and his group's pioneering work led to the use of XPS as a quantitative chemical analysis technique which they dubbed Electron Spectroscopy for Chemical Analysis (ESCA). A vast amount of the group's related research is summarized in two books used by many as standard references. ${ }^{41,42}$ His work revolutionizing the field of PES was rewarded with the Nobel prize in 1981.

\subsection{Angular resolution}

The photoemission-based techniques used by Siegbahn to investigate materials' core levels can also be used to study the valence band, as suggested in Figure 2.4. This was first shown by Berglund and Spicer in 1964 when they identified the 3d DOS in Ag and $\mathrm{Cu} .{ }^{43,44,45}$ The electronic states in dispersive valence bands are well defined in both energy and momentum. Because momentum is conserved in optical excitation during the photoemission process, one can consider attempting to measure both the binding energy and momentum, or $\mathbf{k}$ vector, of the electronic states in the valence bands. This is exactly what is done in an Angle-Resolved Photoemission Spectroscopy (ARPES) experiment, which allows studying an electronic structure's energy and momentum dependencies.

Although simple in concept, the actual photoemission process is extremely complicated, and simplifications and assumptions must be made to interpret the data for the experiment to be worthwhile. The good news is that in a vast number of situations, these simplifications 
and assumptions work quite well and allow us direct access to pertinent information about a material's electronic structure. To be as realistic as possible, the processes of electron photoexcitation, travel to and through the sample surface, and travel to ultimate detection in an analyzer should all be treated as a single coherent process. This type of model is called the "one-step model" and can be quite difficult to work with. Instead, the ARPES experiment is frequently discussed in the popular "three-step model", which can often be accurate enough for many situations and is far more tractable. The three steps are:

\section{Photoexcitation of electrons in the solid}

2. Transport of the electron through the solid to the surface

3. Escape of the electron through the surface into vacuum.

The total measured intensity will then be proportional to the product of the individual probabilities of each step.

The first step is the most interesting as it contains all the information about the intrinsic electronic structure that we are after. The momentum of the photons in the energy ranges typically used for ARPES (20 - several hundred eV) is negligible, and so the photoexcitation is an optical, or direct, transition. These transitions are also called "vertical" transitions, as a plot of energy versus momentum has the electron moving vertically up the plot while conserving momentum. A photon of energy $h \nu$ is incident on the sample and has a finite probability of being absorbed by an electron. The photon may travel some finite distance before being absorbed. Once it is absorbed, the electron is optically excited into a state with $h \nu$ more energy, while maintaining its initial momentum. This process is shown schematically in Figure 2.6(a). Note that the total energy and momentum are conserved. We will refer to the electronic state before excitation as the initial state, and the state right after excitation as the final state. If the initial state is characterized by energy $\left|E_{B}\right|$ and momentum $\mathbf{k}_{i}$, and the final state is characterized by $E_{f}$ (referenced to the vacuum level) 

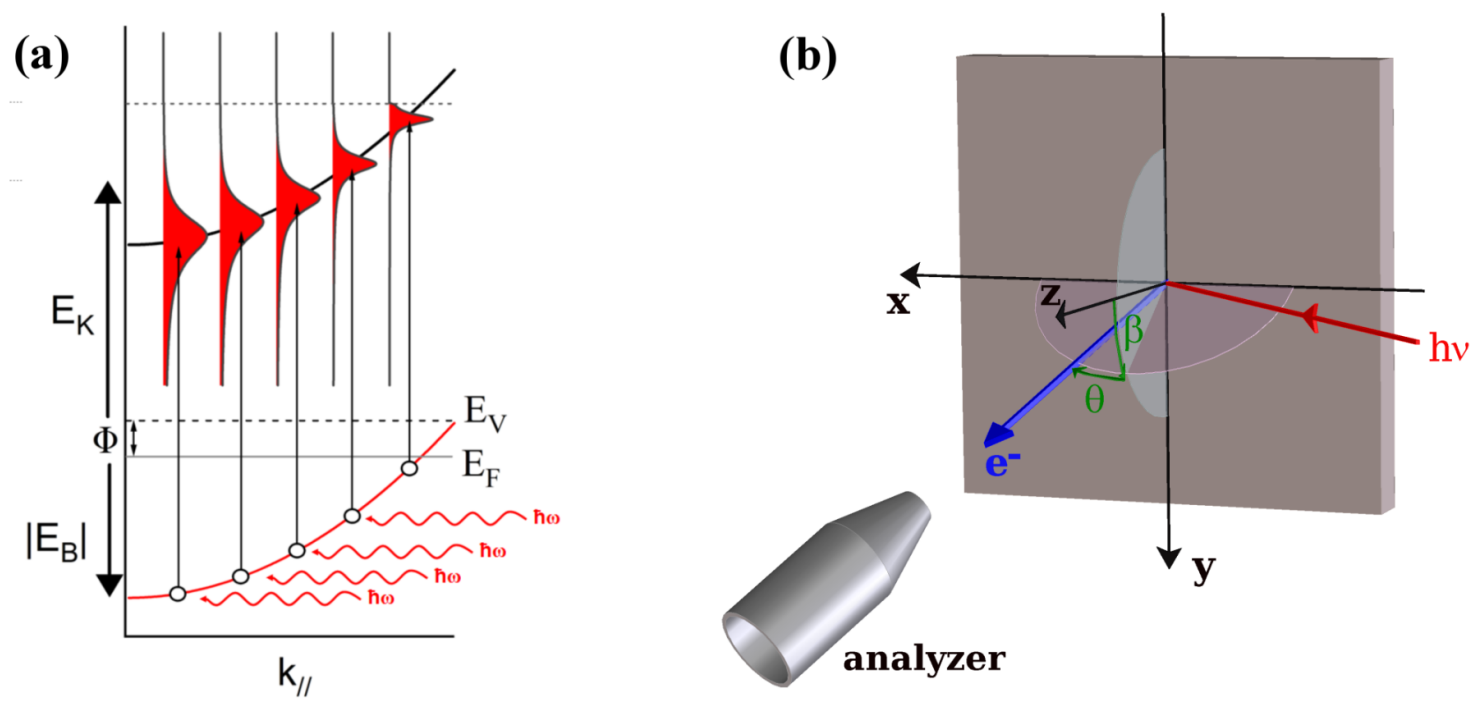

Figure 2.6. (a) Optical transitions from a band in the sample to vacuum. Energy and in-plane momentum are conserved. (b) Geometry of an ARPES experiment. Angles $\theta$ and $\beta$ are defined to correspond to the rotational axes of sample manipulator in our laboratory.

and $\mathbf{k}_{f}$, then we can write

$$
\begin{aligned}
& E_{f}=h \nu-\left|E_{B}\right|-\Phi \\
& \mathbf{k}_{f}=\mathbf{k}_{i}
\end{aligned}
$$

and expand the last line to

$$
\begin{aligned}
k_{x, f} & =k_{x, i} \\
k_{y, f} & =k_{y, i} \\
k_{z, f} & =k_{z, i}
\end{aligned}
$$

In the second step, the excited final-state electrons travel to the surface. Of course, nothing is special about the surface direction, and the final-state electrons may travel away from the surface as well; the only electrons which we have a chance to measure are the fraction which travel to the surface. During transport, the final-state electrons may scatter elastically or inelastically one or multiple times before making it out of the surface. The mean free path of final-state electrons is often quite short, and is dependent on their kinetic energy. It happens that the energy dependent mean free paths for a wide variety of materials 


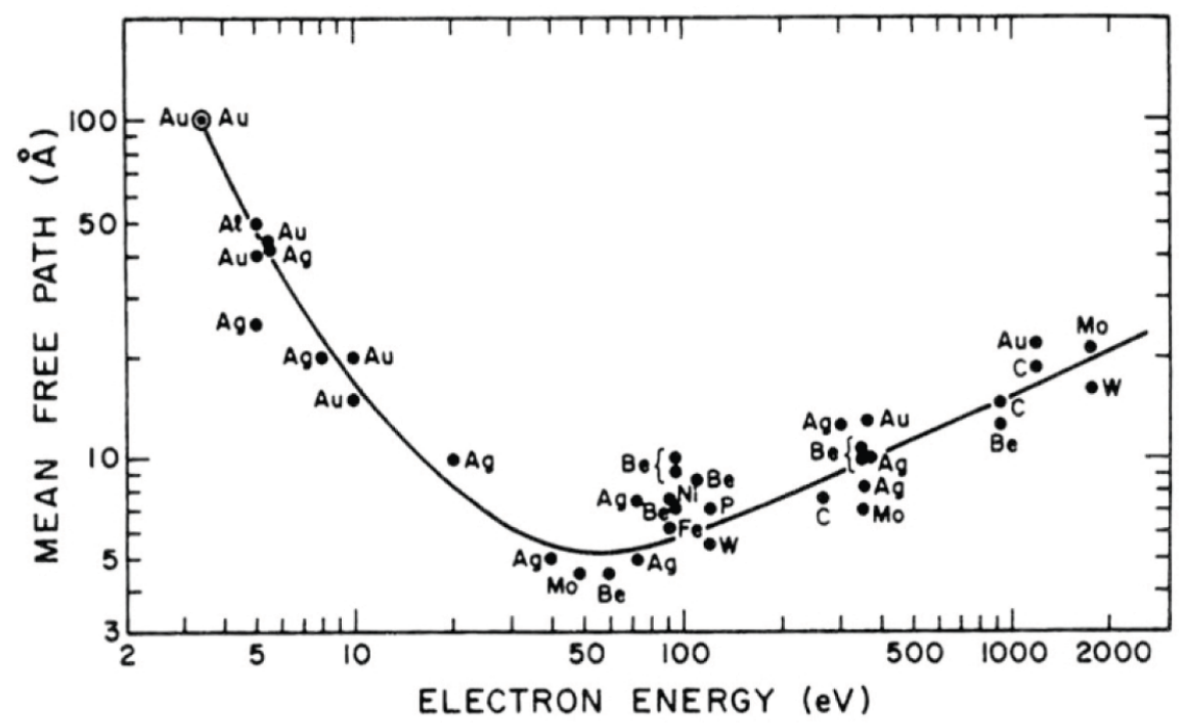

Figure 2.7. "Universal Curve" of inelastic mean free paths of electrons in various materials versus kinetic energy. Figure taken from Ref 26.

follows a similar trend forming the so-called "universal curve" shown in Figure 2.7. Note that there is a minimum of under $5 \AA$ at kinetic energies around $50 \mathrm{eV}$. Therefore, the valence band electrons of interest are going to be near this minimum with the photon energy range of typical ARPES experiments. Because the final-state electrons on average can only travel a couple unit cells without suffering an inelastic collision, ARPES is a very surface sensitive technique - the vast majority of electrons that make it out of the sample without losing energy come from the top one or two atomic layers. This makes the technique very powerful for studying surface physics. Even though the technique is sampling electrons only from the surface, their initial state wavefunctions may extend through the bulk, allowing ARPES to sample bulk electronic structure as well. Deciding whether a certain property in the acquired data is a bulk or surface effect is often challenging, and comparing data with theory can be useful.

In the third step, the final-state electrons must pass through a potential step at the surface. Due to the translational symmetry being maintained along the in-plane directions at the surface, $\mathbf{k}_{\|}$is conserved. Momentum is not maintained along the $\mathbf{z}$-direction, however, because of the potential step in that direction. Once out of the sample and in vacuum, the 
electron will travel to the detector as a well-approximated free-electron plane wave. If we label the kinetic energy of this plane wave $E_{K}$ and its momentum $\mathbf{k}_{\text {out }}$, then we have for this third step

$$
\begin{aligned}
E_{K} & =E_{f} \\
k_{x, \text { out }} & =k_{x, f} \\
k_{y, \text { out }} & =k_{y, f} \\
k_{z, \text { out }} & \neq k_{z, f} .
\end{aligned}
$$

Because the electron is now in a free-electron plane wave on its way to the detector, we also have

$$
E_{K}=\frac{\hbar^{2}}{2 m_{e}}\left(\mathbf{k}_{\text {out }}^{2}\right)=\frac{\hbar^{2}}{2 m_{e}}\left(k_{x, \text { out }}^{2}+k_{y, \text { out }}^{2}+k_{z, \text { out }}^{2}\right) .
$$

By measuring the photoelectron's energy and angles of emission, we can work out the individual components of $\mathbf{k}_{\text {out }}$. The geometry of an Angle-resolved Photoemission Spectroscopy (ARPES) experiment is illustrated in Figure 2.6(b). The slightly unconventional angles (not typical spherical coordinates!) are used to match the rotational axes of the sample manipulator in our laboratory. From equation 2.6 we have

$$
\begin{aligned}
k_{x, \text { out }} & =\frac{1}{\hbar} \sqrt{2 m_{e} E_{K}} \sin \theta \\
k_{y, \text { out }} & =\frac{1}{\hbar} \sqrt{2 m_{e} E_{K}} \cos \theta \sin \beta \\
k_{z, \text { out }} & =\frac{1}{\hbar} \sqrt{2 m_{e} E_{K}} \cos \theta \cos \beta .
\end{aligned}
$$

Combined with equations 2.3-2.5, we can then write for the initial states

$$
\begin{aligned}
\left|E_{B}\right| & =h \nu-\Phi-E_{K} \\
k_{x, i} & =0.512 \sqrt{E_{K}} \sin \theta \\
k_{y, i} & =0.512 \sqrt{E_{K}} \cos \theta \sin \beta
\end{aligned}
$$

where $k_{x, y, i}$ are in units of $\AA^{-1}, E_{K}$ is in units of $\mathrm{eV}$, and $\frac{\sqrt{2 m_{e}}}{\hbar}$ is approximated as 0.512 . So we see that by resolving the kinetic energies and angles of emission of the photoelectrons, they can be mapped back to the initial states' binding energy and in-plane momentum. For 
2-dimensional systems such as surface states or quasi-2D materials, this gives a complete determination of the (spin-integrated) electronic states. This makes such 2-dimensional systems ideal for ARPES experiments.

Full 3-dimensional systems are more complicated, in part because of the loss of $k_{z}$ conservation through the surface in step 3 . Unfortunately, this means that $k_{z}$ cannot be directly extracted from the measurements of $E_{K}, \theta$, and $\beta$ alone, and proper determination requires complete knowledge of the exact "final states" (the unoccupied bandstructure above $\mathrm{E}_{F}$ ) the electrons are photoexcited to before exiting the sample. Often times, the final state dispersions are approximated as parabolic free-electrons. This technique becomes more appropriate at high kinetic energies (thus high photon energies) where effects of the crystal potential are weaker. In the free-electron approximation, the states are assumed to be free but in an extra potential, $V_{i n}$, called the inner potential which can be thought of as the potential step the electrons must pass through to exit the sample. Thus, going back the first step we have

$$
E_{f}=\frac{\hbar^{2}}{2 m_{e}}\left(k_{x, f}^{2}+k_{y, f}^{2}+k_{z, f}^{2}\right)-V_{i n}
$$

Solving for $k_{z, f}$ and using equations 2.3 and 2.4, the initial momentum along $\mathrm{z}$ is

$$
k_{z, i}=\sqrt{\frac{2 m_{e}}{\hbar^{2}}\left(h \nu-\Phi-\left|E_{B}\right|+V_{i n}\right)-k_{x, i}^{2}-k_{y, i}^{2}} .
$$

Experimentally, $V_{i n}$ can be determined by looking at normal emission $\left(\theta=\beta=0=k_{x, y, i}\right)$ and varying the photon energy, $\hbar \omega$. $V_{i n}$ can then be solved for by fitting the periodicity to the known Brillouin zone size.

The $k_{z}$ component causes other complications for ARPES experiments. As mentioned earlier, the final-state electrons have short inelastic mean free paths as they travel through the solid. This concept can also be described by a finite photoelectron lifetime. A way to express this lifetime is through an imaginary part to the momentum, implying a finite broadening to $\mathbf{k}_{f}$. Again, due to the surface discontinuity in the z-direction, only $k_{z}$ can have an imaginary component, giving a broadening of

$$
\Delta k_{z, f}=\frac{1}{\tau_{e} v_{e, z}}
$$




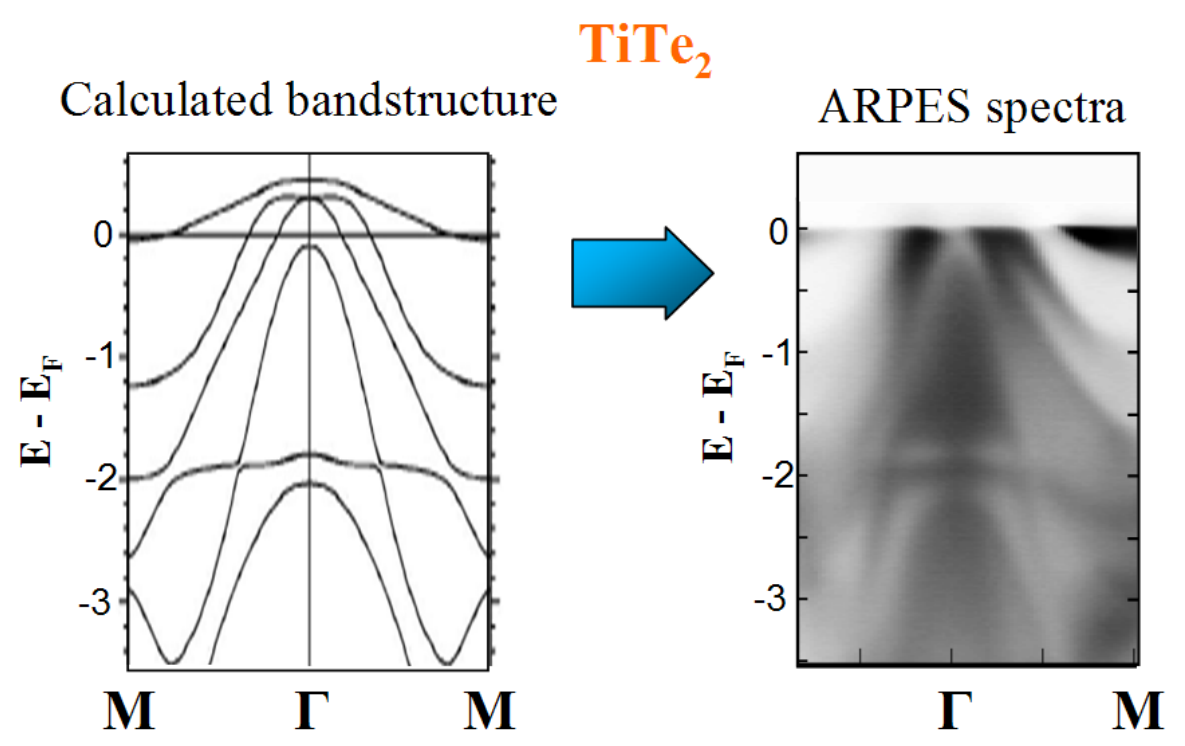

Figure 2.8. Example of ARPES bandmapping of the quasi-2D layered $\mathrm{TiTe}_{2}$. Bandstructure calculations along the $\Gamma \mathrm{M}$ direction, adapted from ref. 47, are show on the left. False-color plot (maximum intensity is dark) of ARPES intensity along same direction is shown on the right.

where $\tau_{e}$ is the photoelectron lifetime and $v_{e, z}$ is the photoelectron, or final-state, band velocity along z. This "wiggle-room" in the required vertical transition of the optical excitation implies an unavoidable integration over $k_{z, i}$; i.e. $\Delta k_{z, f}=\Delta k_{z, i}=\Delta k_{z}$. If there is not much dispersion along $k_{z, i}$ in the neighborhood of the Brillouin zone being probed, this is not as severe a problem. Viewed in another way, the $k_{z}$ broadening implies an energy broadening in the measured spectrum of

$$
\Delta E_{K} \approx v_{h, z} \Delta k_{z}=\frac{v_{h, z}}{v_{e, z}} \frac{1}{\tau_{e}}
$$

where $v_{h, z}$ is the "hole", or initial state, band velocity along z. Again, this shows that if $v_{h, z}$ is small, such as with surface states and quasi-2D materials, this will be a small effect. An excellent discussion of $k_{z}$-broadening of ARPES spectra is found in ref. 46 .

Thus we find that by resolving both energy and angle in an ARPES experiment, effective bandmapping can be done (even in the $3 \mathrm{D}$ case, although it is more difficult) by tracking the local ARPES intensity maxima in $E_{B}$ as a function of emission angles and equating these to the bandstructure $E_{n}(\mathbf{k})$. Additionally, by mapping the ARPES intensity maxima at $\mathrm{E}_{F}$ as a function of $\mathbf{k}$, detailed information about the Fermi surface topology can be directly 
accessed. These two concepts make ARPES an extremely powerful tool. An example of bandmapping is shown for the layered material, $\mathrm{TiTe}_{2}$, in Figure 2.8. The ARPES intensity map on the right shows strong agreement with the calculated bandstructure on the left, illustrating the direct bandmapping ability of ARPES. This concept was introduced in 1964 by Kane, ${ }^{48}$ but was initially received negatively. In 1974, however, ARPES bandmapping was first performed and directly compared to calculated bandstructures in a number of great works by Smith, Traum, and DiSalvo. ${ }^{49,50,51}$. Already understanding the simplifications of performing ARPES on quasi-2D systems, these initial works were done on the layered $\mathrm{TaS}_{2}$ and $\mathrm{TaSe}_{2}$. With additional improvements in instrumental resolutions and efficiencies, ARPES has since become an extremely powerful tool for directly probing a material's bandstructure.

\subsection{Single particle spectral function}

In the previous section, it was shown that because of the conservation of energy and momentum, ARPES is able to map the basic bandstructure of solids. It should be noted that the photoemission process starts with a system of $N$ electrons and removes one of them, leaving an excited $N-1$ system. With a more rigorous investigation of the photoexcitation step (step-1 of the three-step model, where the information about the intrinsic electronic structure is contained), it can be shown that there is a wealth of details about the manybody response of the $N-1$ system in an ARPES experiment. Other sources give detailed reviews of this analysis, ${ }^{30,26}$ so only a brief outline need be given here.

The transition rate of step- 1 from an initial N-electron state, $\left|\Psi_{i}^{N}\right\rangle$, to a final N-electron state, $\left|\Psi_{f}^{N}\right\rangle$, can be given by Fermi's Golden Rule as

$$
w_{f i}=\frac{2 \pi}{\hbar}\left|\left\langle\Psi_{f}^{N}\left|H_{r a d}\right| \Psi_{i}^{N}\right\rangle\right|^{2} \delta\left(E_{f}^{N}-E_{i}^{N}-h \nu\right)
$$

where $H_{\text {rad }}$ is the interaction Hamiltonian of the incident radiation field, the energy of the

initial state is $E_{i}^{N}$, and the energy of the final state is given by $E_{f}^{N}=E_{f}^{N-1}+E_{K}$. Here we define $E_{f}^{N-1}$ to be the energy of the remaining $N-1$ state with $E_{K}$ still the energy of the photoemitted electron. 
To investigate the properties of the $N-1$ excited state, we can begin by decomposing $\Psi_{f}^{N}$ into a product of $\Psi_{f}^{N-1}$ and the wavefunction of the outgoing photoelectron with wavevector $\mathbf{k}, \phi_{f}^{\mathbf{k}}$

$$
\Psi_{f}^{N}=\mathcal{A} \phi_{f}^{\mathbf{k}} \Psi_{f}^{N-1}
$$

where $\mathcal{A}$ is the antisymmetrization operator enforcing the fermion Symmetrization Postulate (section 1.2.4). This decomposition is only valid assuming the ejected photoelectron leaves the system quickly enough so that it and the remaining $N-1$ system do not have a chance to interact. This is known as the "sudden approximation", and as it is our first step here, it is clearly important for the present model of understanding ARPES. It is normally assumed to be valid for photoelectrons with $E_{K} \gtrsim 20 \mathrm{eV}$. ARPES spectra taken with photon energies less than this are then subject to concern regarding the sudden approximation, although it seems that at least in the HTC cuprate systems it remains valid to very low photon energies $(6 \mathrm{eV}) .{ }^{52}$ The final state wavefunction, $\Psi_{f}^{N-1}$ is taken to be an eigenfunction of the excited state, $\Psi_{m}^{N-1}$, with energy $E_{m}^{N-1}$, so that equation 2.14 can be written as

$$
\Psi_{f}^{N}=\mathcal{A} \phi_{f}^{\mathbf{k}} \Psi_{m}^{N-1}
$$

Next, a similar approach can be taken to decompose the initial state, $\Psi_{i}^{N}$, into a product of $\Psi_{i}^{N-1}$ and a one electron orbital, $\phi_{i}^{\mathbf{k}}$, of the electron to be photoemitted. This can be written

$$
\Psi_{i}^{N}=\mathcal{A} \phi_{i}^{\mathbf{k}} \Psi_{i}^{N-1}
$$

The $\Psi_{i}^{N-1}$ should be understood to be simply the result of removing one electron from the $N$-electron wavefunction, which can be expressed as

$$
\Psi_{i}^{N-1}=c_{\mathbf{k}} \Psi_{i}^{N}
$$

where $c_{\mathbf{k}}$ is the annihilation operator of an electron at $\mathbf{k}$. This stresses that $\Psi_{i}^{N-1}$ is not in general an eigenfunction of the $N-1$ system.

Combining equations 2.15 and 2.16, we can write the matrix element in equation 2.13 as

$$
\left\langle\Psi_{f}^{N}\left|H_{\text {rad }}\right| \Psi_{i}^{N}\right\rangle=\left\langle\phi_{f}^{\mathbf{k}}\left|H_{\text {rad }}\right| \phi_{i}^{\mathbf{k}}\right\rangle\left\langle\Psi_{m}^{N-1} \mid \Psi_{i}^{N-1}\right\rangle
$$


The $\left\langle\phi_{f}^{\mathbf{k}}\left|H_{\text {rad }}\right| \phi_{i}^{\mathbf{k}}\right\rangle$ term is simply the one-electron dipole matrix element and can be represented as $\mathcal{M}_{f, i}^{\mathbf{k}}$. The total photoemission current, $I\left(\mathbf{k}, E_{K}\right)$ will be proportional to the sum of possible transition rates, $\sum_{f, i} w_{f i}$, where $w_{f i}$ must also be summed over all possible excited eigenstates, $m$. Combining equations 2.13 and 2.18 thus gives us

$$
I\left(\mathbf{k}, E_{K}\right) \propto \sum_{f, i}\left|\mathcal{M}_{f, i}^{\mathbf{k}}\right|^{2} \sum_{m}\left|c_{m, i}\right|^{2} \delta\left(E_{K}+E_{m}^{N-1}-E_{i}^{N}-h \nu\right) .
$$

In the above, $\left|c_{m, i}\right|^{2}=\left.\left\langle\left|\Psi_{m}^{N-1}\right| \Psi_{i}^{N-1}\right\rangle\right|^{2}$ represents the total probability that removing the electron from the $N$-electron state $i$, will result in the excited eigenstate $m$ of the $N-1$ electron system. Note that the binding energy of the electron is the difference in energies of the $N-1$ and $N$-electron systems, $\left|E_{B}\right|=E_{m}^{N-1}-E_{i}^{N}$. Thus the Dirac delta function in equation 2.19 enforces the energy conservation used in previous sections (the work function is not written here as this analysis only involves the first step of the three step model, and references $E_{K}$ to the Fermi level instead of the vacuum level). If the $N$ electrons of the system are independent and non-interacting, $\Psi_{i}^{N-1}$ is in fact an eigenfunction of the $N-1$ excited state, and thus $c_{m, i}=1$ for one value of $m$ and 0 for all others. Assuming the $\mathcal{M}_{f, i}^{\mathbf{k}} \neq 0$, this means the resulting ARPES spectra will be a series of delta functions at $\left|E_{B}^{\mathbf{k}}\right|=\epsilon_{\mathbf{k}}$, the Hartree-Fock energies, as depicted in the left panel of Figure 2.9. If there are many-body interactions present, however, many $c_{m, i}$ will have non-zero contributions, resulting in ARPES spectra consisting of a main line and a spread of satellite excitations, as depicted in the right panel of Figure 2.9.

When studying the physics of interacting many-body problems, a popular approach is to use the Green's function formalism ${ }^{53}$ where the one-electron propagation in the many-body system can be described by the time-ordered Green's function, $\mathcal{G}\left(t-t^{\prime}\right)$. We can define the single particle spectral function, $A(\mathbf{k}, \omega)$, as the imaginary part of the Fourier transformed Green's function as

$$
A(\mathbf{k}, \omega)=-\frac{1}{\pi} \operatorname{Im} G(\mathbf{k}, \omega)
$$

where we also have the one electron removal single particle spectral function given by

$$
A(\mathbf{k}, \omega)=\sum_{m}\left|\left\langle\Psi_{m}^{N-1}\left|c_{\mathbf{k}}\right| \Psi_{i}^{N}\right\rangle\right|^{2} \delta\left(\omega-E_{m}^{N-1}+E_{i}^{N}\right)
$$




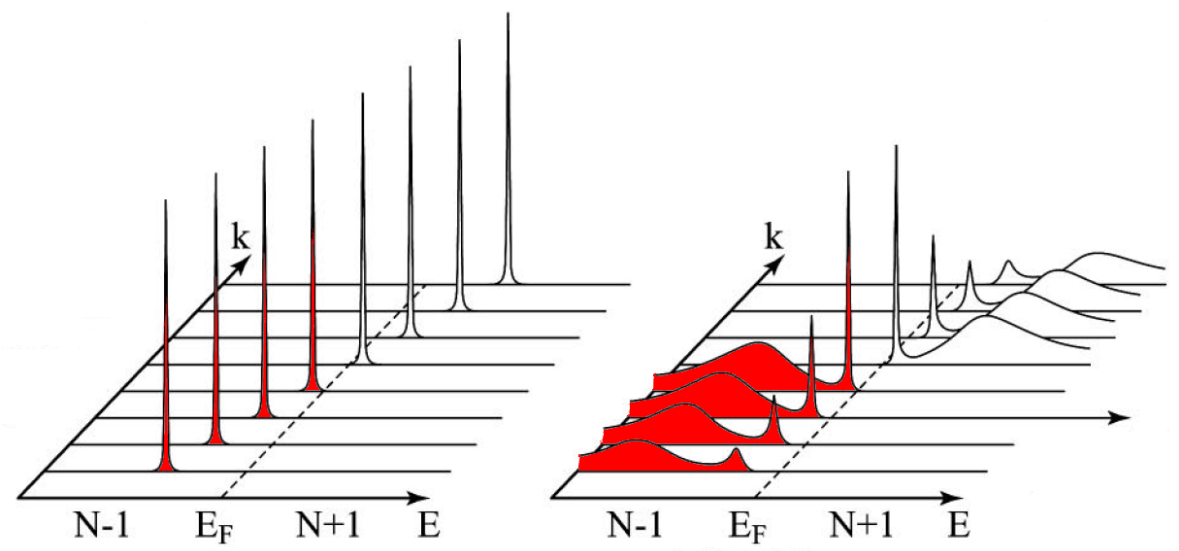

Figure 2.9. Example one-electron removal and addition spectra for a non-interacting (left) and an interacting (right) system consisting of a single band crossing $\mathrm{E}_{F}$. ARPES spectra correspond to the one-electron removal spectra and only access the occupied part, highlighted in red. Figure adapted from ref. 30.

This of course is mainly just the $\left|c_{m, i}\right|$ part of equation 2.19, which we can then re-write as

$$
I(\mathbf{k}, \omega) \propto \sum_{f, i}\left|\mathcal{M}_{f, i}^{\mathbf{k}}\right|^{2} f(\omega) A(\mathbf{k}, \omega) \delta\left(\omega+E_{K}-h \nu\right)
$$

where we have also added the Fermi function, $f(\omega)$ to restrict the measured intensity to the occupied states extended to finite temperature.

For a non-interacting system, the Green's function is given by

$$
G(\mathbf{k}, \omega)=\frac{1}{\omega-\epsilon_{\mathbf{k}}-i \eta}
$$

where $\eta \rightarrow 0$. Equation 2.20 then gives

$$
A(\mathbf{k}, \omega)=-\frac{1}{\pi} \delta\left(\omega-\epsilon_{\mathbf{k}}\right) .
$$

Plugging this back into equation 2.22 reaffirms the early statement about the ARPES spectra being delta functions as in the left side of Figure 2.9. For an interacting system, the interactions can be formally introduced as a complex electron self-energy usually written as $\Sigma(\mathbf{k}, \omega)=\Sigma^{\prime}(\mathbf{k}, \omega)+i \Sigma^{\prime \prime}(\mathbf{k}, \omega)$. In this case, the Green's function is

$$
G(\mathbf{k}, \omega)=\frac{1}{\omega-\epsilon_{\mathbf{k}}-\Sigma(\mathbf{k}, \omega)}
$$

with the spectral function then given by

$$
A(\mathbf{k}, \omega)=-\frac{1}{\pi} \frac{\Sigma^{\prime \prime}(\mathbf{k}, \omega)}{\left(\omega-\epsilon_{\mathbf{k}}-\Sigma^{\prime}(\mathbf{k}, \omega)\right)^{2}+\left(\Sigma^{\prime \prime}(\mathbf{k}, \omega)\right)^{2}} .
$$


Thus, high resolution ARPES data does not only function as effective bandmapping, but also allows direct measurement of many-body effects such as the real and imaginary selfenergies in the spectral function.

\subsection{EDC and MDC analysis}

For an ARPES experiment, a dataset typically consists of a multi-dimensional map of photoemission intensity as a function of $\mathbf{k}$ (i.e. $k_{x}, k_{y}$, and/or $k_{z}$ ) and binding energy, $\left|E_{B}\right|$. Usually, the minimal dataset is a 2-dimensional map as a function of $|\mathbf{k}|$ along some desired direction in $k$-space and binding energy so that the band dispersion along that direction can be mapped and its single particle spectral function along that direction can be analyzed. There are two standard ways of analyzing such a dataset. One is to plot the intensity as a function of energy at fixed $|\mathbf{k}|$ values, and study the resulting "energy distribution curves" (EDCs) at numerous values of momentum. The other is to plot the intensity as a function of momentum at fixed $\left|E_{B}\right|$ values, and study the resulting "momentum distribution curves" (MDCs) at numerous values of binding energy. An example of an EDC and an MDC from such a 2D ARPES dataset is shown in Figure 2.10(a), with EDC and MDC analyses shown in (b) and (c) respectively. The band dispersion can be extracted from the peak positions (green circles) in either method, although MDCs are generally more accurate with steeper dispersions, and EDCs are generally more accurate near the bottom and tops of band edges.

The EDC is the more traditional analysis technique and gives a global view of the spectral function's energy dependence. It is useful as it can establish the existence of important features such as quasiparticle peaks ${ }^{54}$ and gaps. Information about the particular manybody interactions present can be found in rigorous analysis of the EDC lineshape. However, EDCs are often difficult to work with because they tend to have complex lineshapes. Near $\mathrm{E}_{F}$, where most of the interest is anyway, the EDC lineshape is additionally complicated by the modulation by the Fermi function, $f(\omega)$. The inelastic and secondary background can also add complication as its proper removal is not straightforward with no general theoretical model to use. Note that the disturbance due to $f(\omega)$ is clearly seen as the Fermi 

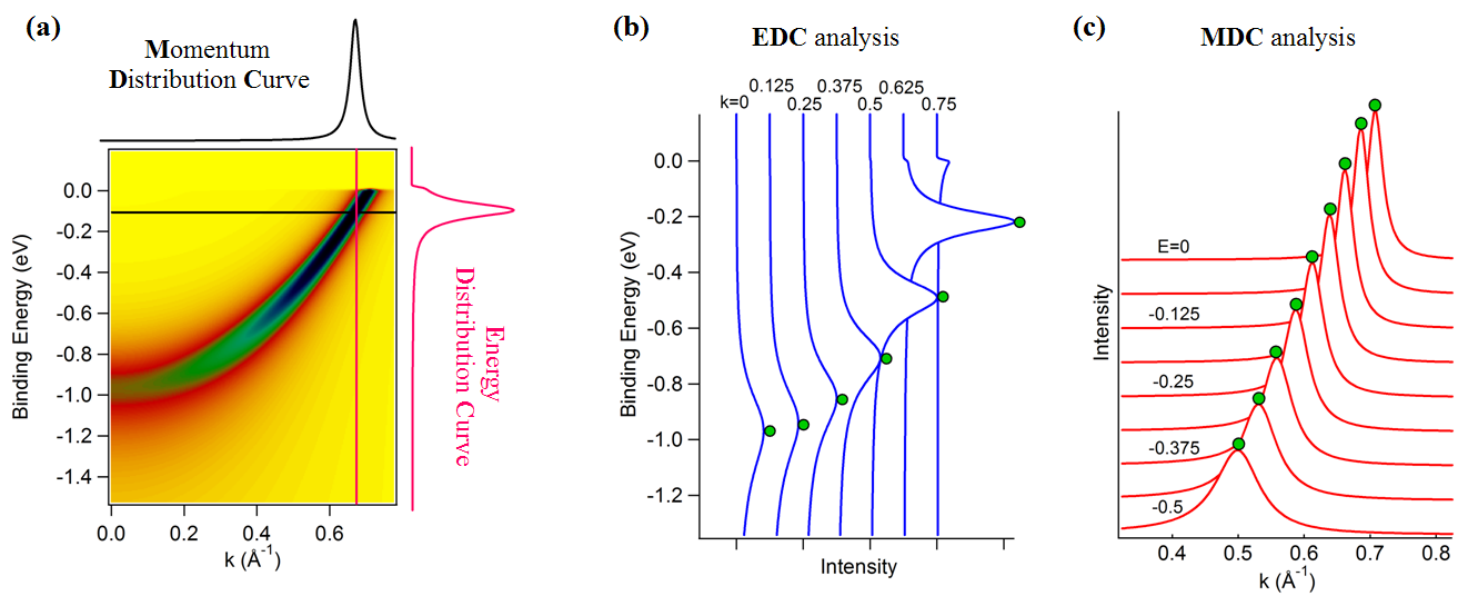

Figure 2.10. (a) False-color plot of simulated ARPES intensity map of a parabolic Fermi liquid band. MDC and EDC concept is illustrated. (b) EDC analysis of a stack of EDCs. (c) MDC analysis of a stack of MDCs.

edge in the last couple EDC's of Figure 2.10(b), but the disturbance due to the usual strong inelastic background is not present in this simulation.

The MDC offers a number of simplifications. At constant binding energy, the Fermi function and the inelastic background are no longer issues as they are both momentum independent. This tends to make MDC lineshapes easier to work with and are often straightforwardly fit as Lorentzians. Extracting the band dispersion from an MDC analysis (as in Figure $2.10(\mathrm{c})$ ) is usually not difficult; the resulting dispersion, $\omega(\mathbf{k})$, can be compared to some non-interacting bare band model $\epsilon(\mathbf{k})$ to extract $\Sigma^{\prime}\left(\mathbf{k}, \omega_{\mathbf{k}}\right)$ as well as information about the lifetime. MDC analysis has become standard for investigating many-body interactions in detail. ${ }^{55,56} \mathrm{~A}$ much more detailed review of the two methods and their direct relations to the single particle spectral function are given in ref. 57 .

\subsection{Dipole matrix elements}

In the analysis of section 2.4 we gave no details about the actual photoemitting Hamiltonian in equation 2.13. Ignoring electron spin, the Hamiltonian in the radiation field can 
be given by

$$
H=\frac{1}{2 m_{e}}\left(\mathbf{p}-\frac{e}{c} \mathbf{A}\right)^{2}+e \phi
$$

where $\mathbf{A}$ is the vector potential and $\phi$ is the scalar potential, which by proper choice of gauge can be made zero. This then reduces to the perturbation Hamiltonian to be used in equation $2.13, H_{r a d}$, given by

$$
\begin{aligned}
H_{r a d} & =-\frac{e}{2 m_{e} c}(\mathbf{A} \cdot \mathbf{p}+\mathbf{p} \cdot \mathbf{A}) \\
& \approx-\frac{e}{m_{e} c} \mathbf{A} \cdot \mathbf{p}
\end{aligned}
$$

where the term quadratic in $\mathbf{A}$ is ignored, the commutator $[\mathbf{p}, \mathbf{A}]=-i \hbar \nabla \cdot \mathbf{A}$ is used, and the dipole approximation is made in which $\nabla \cdot \mathbf{A}=0$ (valid in the UV range, except for near the surface where possibly strong spatial variations in the field may result in indirect transitions as well). Hence, the matrix elements, $\mathcal{M}_{f, i}^{\mathbf{k}}$ in equations 2.19 and 2.22 are

$$
\mathcal{M}_{f, i}^{\mathbf{k}} \propto\left\langle\phi_{f}^{\mathbf{k}}|\mathbf{A} \cdot \mathbf{p}| \phi_{i}^{\mathbf{k}}\right\rangle
$$

This expression can be further simplified by expressing the radiation field, $\mathbf{A}$, as a plane wave with $\mathbf{A} \sim A_{0} \hat{\boldsymbol{\epsilon}} e^{i(\omega / c) \hat{\mathbf{n}} \cdot \mathbf{x}}$, where $\hat{\boldsymbol{\epsilon}}$ is a unit vector along the direction of the light polarization, and $\hat{\mathbf{n}}$ is the unit vector along the light propogation direction. The exponential term can be expanded in a power series; in the standard electric dipole (E1) approximation only the first term, 1, is kept. This gives $\mathbf{A} \sim A_{0} \hat{\boldsymbol{\epsilon}}$. By making use of the commutation relation of the full Hamiltonian, $[\mathbf{x}, H]=i \hbar \mathbf{p} / m_{e}$, equation 2.30 can be reduced to

$$
\mathcal{M}_{f, i}^{\mathbf{k}} \propto\left\langle\phi_{f}^{\mathbf{k}}|\hat{\boldsymbol{\epsilon}} \cdot \mathbf{x}| \phi_{i}^{\mathbf{k}}\right\rangle .
$$

This is the standard dipole transition and so $\mathcal{M}_{f, i}^{\mathbf{k}}$ becomes subject to the standard dipole selection rules (noting that $\mathbf{x}$ is a spherical tensor of rank 1).

In some situations, a simple symmetry analysis shows strong "matrix element effects" where $\mathcal{M}_{f, i}^{\mathbf{k}}$ strongly modulates the ARPES intensity. ${ }^{30}$ For instance, consider even/odd symmetry with respect to the plane formed by the incident light vector and outgoing photoelectron vector. The $\hat{\boldsymbol{\epsilon}} \cdot \mathbf{x}$ will be even $(+)$ with respect to reflection about this plane for p-polarized light and odd (-) for s-polarized light. Detectable photoelectrons $\left(\phi_{f}^{\mathbf{k}}\right)$ must 
have even $(+)$ wavefunctions with respect to the plane (or would have nodes, 0 probability amplitude, at the detector). For p-polarized light, the matrix element then goes as $\langle+|+|$ ? $\rangle$. This matrix element will then be maximum for initial states of even spatial symmetry with respect to the defined plane, and will in fact be zero for initial states of odd symmetry. For s-polarized light, the situation is reversed as the matrix element will go as $\langle+|-|$ ? $\rangle$.

If we take the photoelectron wavefunction, $\phi_{f}^{\mathbf{k}}$, to be a plane wave, $e^{i \mathbf{k} \cdot \mathbf{x}}$, the matrix element from equation 2.30 can be expressed as

$$
\begin{aligned}
\left|\mathcal{M}_{f, i}^{\mathbf{k}}\right|^{2} & \propto\left|\left\langle e^{i \mathbf{k} \cdot \mathbf{x}}\left|A_{0} \hat{\boldsymbol{\epsilon}} \cdot \mathbf{p}\right| \phi_{i}^{\mathbf{k}}\right\rangle\right|^{2} \\
& \propto\left|(\hat{\boldsymbol{\epsilon}} \cdot \mathbf{k})\left\langle e^{i \mathbf{k} \cdot \mathbf{x}} \mid \phi_{i}^{\mathbf{k}}\right\rangle\right|^{2} .
\end{aligned}
$$

This illustrates that the matrix element can be dependent on the initial state wavefuntion as well as the outgoing k-vector, which can be effected by the experiment's geometry. This also demonstrates ARPES photon energy dependence: a change in $h \nu$ will change $E_{K}$ as well as the magnitude of $\mathbf{k}$. The overlap of the outgoing plane wave and the initial state, $\left\langle e^{i \mathbf{k} \cdot \mathbf{x}} \mid \phi_{i}^{\mathbf{k}}\right\rangle$, depends on both details of the initial state wavefunction as well as the outgoing k-vector, thus causing modulations in the intensity with photon energy (not necessarily monotonic). ${ }^{30}$ A great example of photon energy dependent matrix elements in the double layer HTC cuprates is given in ref. 58. One must always remember that ARPES data may be influenced by difficult to predict matrix elements. Sometimes, these matrix element effects can be taken advantage of. In some cases, signal from some bands can be enhanced with respect to others due to different matrix element dependencies on photon energy, ${ }^{58,59,60,61,56}$ photon polarization, ${ }^{62,63}$ or polarization geometry. ${ }^{54}$ Matrix element effects can also be used for aiding in identifying initial state characteristics such as orbital symmetry character. ${ }^{64}$

\subsection{State-of-the-art ARPES}

The ARPES technique has undergone enormous growth in the past decade. Much of the current strength and popularity of ARPES is related to its symbiotic relationship with the field of HTC cuprates. From initial observations of d-wave gap symmetry, ${ }^{65}$ to fine 
kinks in the electronic dispersions, ${ }^{55}$ to anomalous isotope effects, ${ }^{66}$ ARPES has been at the forefront of HTC discoveries. ${ }^{30}$ The intrinsic high interest in the HTCs encouraged a constant push for advancements in ARPES instrumentation and technique, which allowed subsequent discoveries that would not have been otherwise possible. This investment in the development of the technique is now paying enormous dividends by allowing fundamental insights in other material systems as well. ${ }^{67,54,68,69,70}$

With a state-of-the-art system, it is now possible to take a 5-dimensional data set of intensity as a function of $\left|E_{B}\right|, k_{x}, k_{y}, k_{z}$ (or $h \nu$ ), and sample temperature through the entire Brillouin zone within a 24-hour shift, with energy resolutions below $10 \mathrm{meV}$ and momentum resolutions below $1 \%$ of typical Brillouin zones. As part of the work to similarly develop spin-ARPES that is covered in this thesis, a state-of-the-art ARPES system was designed and constructed. Technical details of the ARPES technique will be exposed through a brief summary of this system.

\subsubsection{Spectrometer}

The heart of any photoemission experiment is the instrumentation used to actually measure the energy (and/or angular and/or spin) distribution of the photoelectron intensity. We shall refer to instrumentation which measures the photoelectron intensity as a function of one or more of the above degrees of freedom as an electron spectrometer. An electron spectrometer must include an electron analyzer and an electron detector. The analyzer provides the energy (and/or angular and/or spin) analysis, separating the photoemitted electrons according to kinetic energy (and/or angle of emission and/or spin), while the detector actually records the final presence of a photoelectron. In the case of Millikan's early photoemission experiments ${ }^{34}$ (section 2.1), the spectrometer was a retarding field Faradaycup (detector) which resolved in energy by counting only photoelectrons with energy greater than that needed to cross an adjustable electrostatic retardation field (energy analyzer). In the case of Siegbahn's original $\beta$-decay spectrometer (section 2.2), the energy analyzer was a dispersive magnetic field and the detector was a Geiger-Müller counter. ${ }^{25}$ 
Electron energy analyzers can typically utilize one of three techniques: passing a potential barrier, measuring the time of flight across a given distance, and the deflection in a dispersive field. The potential barrier method is the basis for Millikan's analyzer and is very straightforward, however has a very low signal to noise ratio and cannot be used for high resolution instruments (it is still used in LEED optics to provide rough Auger electron spectroscopy capabilities). The time-of-flight (TOF) method has historically been quite popular with ion mass spectrometers and neutron spectrometers, but not so with electron spectrometers, likely due to the success of the final category. We will return to the TOF technique in Chapter 4. The dispersive field method sends the electrons to be analyzed through a field which spatially deflects the electrons differing amounts dependent on their kinetic energy. A measurement of where the electrons are deflected to constitutes a measurement of their energy. Analyzers of this type built with magnetic fields (such as Siegbahn's $\beta$-decay spectrometer) offer the possibility of high precision with well engineered magnetic fields. However, due to the difficulty of producing and repeatably controlling the magnetic fields, analyzers utilizing purely electrostatic fields have since been the most prevalent.

Electrostatic dispersive energy analyzers have seen the most developments and refinements, and include a wide range of designs. Roy and Tremblay ${ }^{71}$ and Smith and Kevan ${ }^{72}$ give excellent reviews of many general designs and provide a quantitative comparison of effectiveness in terms of energy resolution and transmission efficiency. Here we will only concentrate on the most successful and widely used design, which is historically referred to in the literature as a Spherical Deflector Analyzer (SDA). For a variety of reasons, the SDA makes for a highly effective electron spectrometer for many spectroscopic techniques, and its effectiveness for ARPES is a large part of the technique's success.

The general design of an SDA is depicted in Figure 2.11. The SDA consists of two nested hemispherical electrodes of radius $r_{i n}$ and $r_{\text {out }}$, colored brown and blue, respectively, in the figure. Appropriately applied voltages to the hemispheres will deflect photoelectrons entering through the entrance slit $\left(S_{1}\right)$ on orbits between the hemispheres. The key is that electrons of different kinetic energy within the analyzer take different orbits. Electrons entering the hemispheres with kinetic energy equal to $E_{p}$ will have a circular orbit with radius 


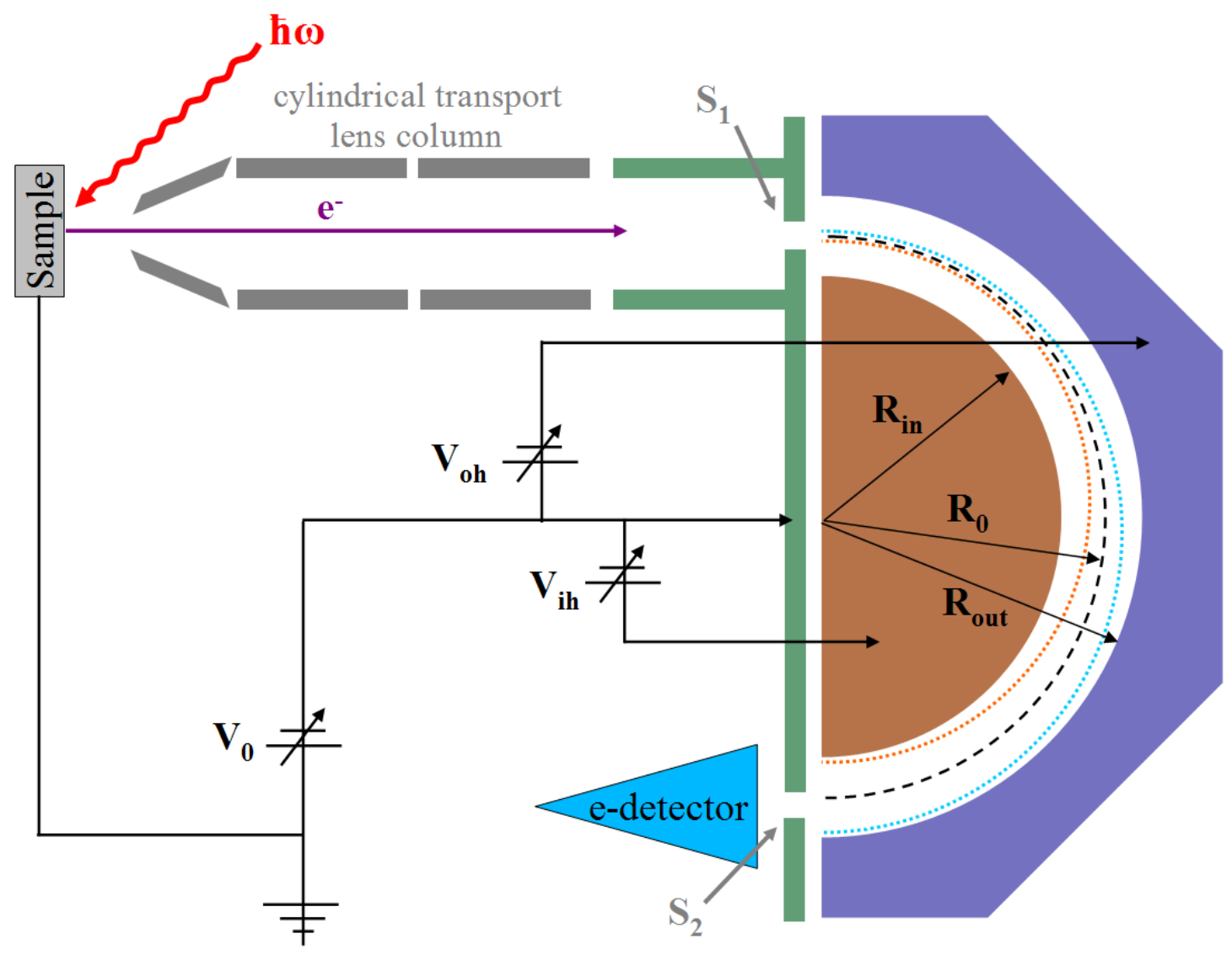

Figure 2.11. Schematic of a SDA, or hemispherical analyzer. Photoelectrons are transported from the sample to the SDA via a cylindrical electrostatic lens column. Electrons with kinetic energy equal to the pass energy $\left(E_{p}\right)$ are deflected on a circular orbit marked by the black dotted line. Electrons with kinetic energy above (below) the pass energy are deflected on larger (smaller) orbits marked by the blue (orange) dotted lines. $S_{1}$ and $S_{2}$ mark the analyzer entrance and exit slits, respectively. 
equal to the central radius through the hemispheres, $r_{0}$ (the black dotted line). Electrons entering the hemispheres with larger or smaller kinetic energies will have orbits of larger or smaller radii, respectively (blue and orange dotted lines). As shown in the figure, only electrons with energy equal to approximately $E_{p}$ will be counted by the electron detector placed after the exit slit, $S_{2}$. Energy analysis is performed through spatial dispersion of the electron beam.

Using the labels of Figure 2.11, the pass energy is given by

$$
E_{p}=e\left(\frac{R_{\text {in }} R_{\text {out }}}{2 R_{0}\left(R_{\text {out }}-R_{\text {in }}\right)}\right) \Delta V
$$

where $\Delta V$ is the potential difference between the inner and outer hemispheres, given by $V_{\text {in }}-V_{\text {out }}$, which is also equal to the power supplies $V_{i h}-V_{\text {oh }}$ in the figure. The "ground plate" (green electrode in Figure 2.11) is set to $V_{0}$, the potential in between the hemispheres at radius $r=r_{0}$, and is the main retardation/acceleration voltage of the spectrometer. The specific voltages applied to the inner and outer hemispheres $\left(V_{\text {in,out }}\right)$ are then

$$
V_{\text {in }, \text { out }}=V_{0}+V_{\text {ih, oh }}=V_{0}+2 \frac{E_{p}}{(-e)}\left(\frac{R_{0}}{R_{\text {in }, \text { out }}}-1\right)
$$

remembering that $e$ is a negative number in this thesis. Electrons entering the spectrometer are retarded or accelerated by $V_{0}$ so that electrons of any initial kinetic energy can be measured with a desired pass energy. Electrons which will make it around the central orbit of the hemispheres will have initial kinetic energy $E_{K}$ given by

$$
E_{K}=E_{p}+e V_{0}
$$

A full spectrum of intensity as a function of $E_{K}$ can be taken in a number of ways. In the most basic mode, the retardation/acceleration voltage, $V_{0}$, is fixed, and the hemisphere voltages can be swept to scan $E_{p}$ and thus $E_{K}$. A slightly more sophisticated mode, called 'Fixed Retardign Ratio', scans the analyzer votages while keeping the retardation ratio (defined as $R=E_{K} / E_{p}$ ) constant. One can also acquire a full spectrum in the so-called 'Fixed Analyzer Transmission' mode which uses a fixed $E_{p}$ (power supplies $V_{i h}$ and $V_{\text {oh }}$ held constant) and scans $E_{K}$ by sweeping $V_{0}$. 
For reference, the electric field and potential between the hemispheres as a function of radial position, $r$, are given by

$$
\begin{aligned}
\mathbf{E}(r) & =\left(\frac{R_{\text {out }} R_{\text {in }}}{R_{\text {out }}-R_{\text {in }}}\right) \frac{\Delta V}{r^{2}} \quad \text { and } \\
V(r) & =V_{0}-\left(\frac{R_{\text {out }} R_{\text {in }}}{R_{\text {out }}-R_{\text {in }}}\right) \frac{\Delta V}{R_{0}}\left(1-\frac{R_{0}}{r}\right) .
\end{aligned}
$$

This expression for the electric field is reassuring as it can easily be seen that a dynamical equilibrium exists for electrons of energy $E_{p}=1 / 2 m_{e} v_{p}^{2}$ to stay on the central orbit with radius $r=R_{0}$ by expressing the force on the electron as $F=e \mathbf{E}\left(R_{0}\right)$ and setting it equal to $m_{e} v_{p}^{2} / R_{0}$.

A key characteristic of an energy analyzer is its energy resolution, $\Delta E$, which is often discussed as a dimensionless fraction of the pass energy referred to as the resolving power. For the SDA, the theoretical resolving power is

$$
\frac{\Delta E}{E_{p}}=\frac{S}{2 r_{0}}+\frac{\alpha^{2}}{4}
$$

where $\mathrm{S}$ is the average slit width of the hemisphere entrance and exit slits, $S=\left(S_{1}+\right.$ $\left.S_{2}\right) / 2$, and $\alpha$ is the accepted half-angle of electrons through the entrance slits in the energy dispersion direction. Disregarding the second term on the right, we can see that there are two ways of improving the resolving power of an SDA - decreasing the entrance/exit slit widths and increasing $r_{0}$ (the size of the instrument). With these fixed physical dimensions, the energy resolution can be improved by working at lower pass energy. Equation 2.38 also shows that the 'Fixed Analyzer Transmission' mode for scanning $E_{K}$ (fixed $E_{p}$, swept $V_{0}$ ) operates with fixed energy resolution throughout the spectrum. Because of this, this is the method typically used for high resolution experiments.

It can also be shown for an SDA that the measured intensity, $I$, at the electron detector is proportional to the pass energy as

$$
I \sim \frac{E_{p}^{2}}{E_{K}}
$$

Therefore we see that improving the energy resolution by reducing $E_{p}$ (equation 2.38) comes at the price of strongly reduced intensity or count rate at the detector, requiring a longer 
acquisition time for a certain level of statistics. As mentioned above, resolution is also improved by reducing the entrance/exit slits widths, but this also of reduces the count rate. As an aside, equation 2.39 shows that operated in the 'Fixed Retardation Ratio' mode, an SDA gives an intensity transmission proportional to $I \sim E_{K}$, while in 'Fixed Analyzer Transmission' mode gives $I \sim 1 / E_{K}$.

As discussed in refs. 72,73, the SDA is a particularly good choice of energy analyzer for an electron spectrometer because of the straightforward use of a position sensitive detector (PSD) to multiplex data acquisition. The SDA is well suited for use with a PSD because the focal surface is a flat plane at the exit of the ground plate. Also, the radial position, $r$, of electrons at the exit of the ground plate is linearly proportional (to first approximation) to its kinetic energy inside the hemispheres, $E=E_{K}-e V_{0}$, as

$$
\frac{r-R_{0}}{R_{0}}=2 \frac{E-E_{p}}{E_{p}}
$$

or

$$
r=2 R_{0} \frac{E}{E_{p}}-R_{0}
$$

A multichannel PSD placed at the rear of the hemispheres instead of an exit slit can then take measurements of intensity at multiple equally spaced $E$ simultaneously. By recording many measurements in parallel, the overall efficiency is improved by a factor of the number of distinct channels used. The increased efficiency can then be used to take a given spectrum with higher resolution by reducing $E_{p}$ or $S_{1}$.

The SDA also focuses in the direction out of the plane of Figure 2.11, meaning arrival positions along an axis out of the figure plane at the final detector are proportional to the position along the out-of-plane direction at the hemisphere entrance slit. Suitable electrostatic cylindrical transport lens columns are capable of directly imaging photoelectron emission angle to out-of-plane position along the analyzer entrance slit. If a 2 dimensional multichannel PSD is placed at the exit of the hemispheres, electron arrival at a channel at position $\mathrm{x}$ and $\mathrm{y}$ on the PSD can be mapped to emission angle $\mathrm{X}$ and kinetic energy $\mathrm{Y}$. This 2D multiplexing is an additional radical improvement in spectrometer efficiency for angle resolved measurements as it multiplexes simultaneous angle measurements, as well. This 


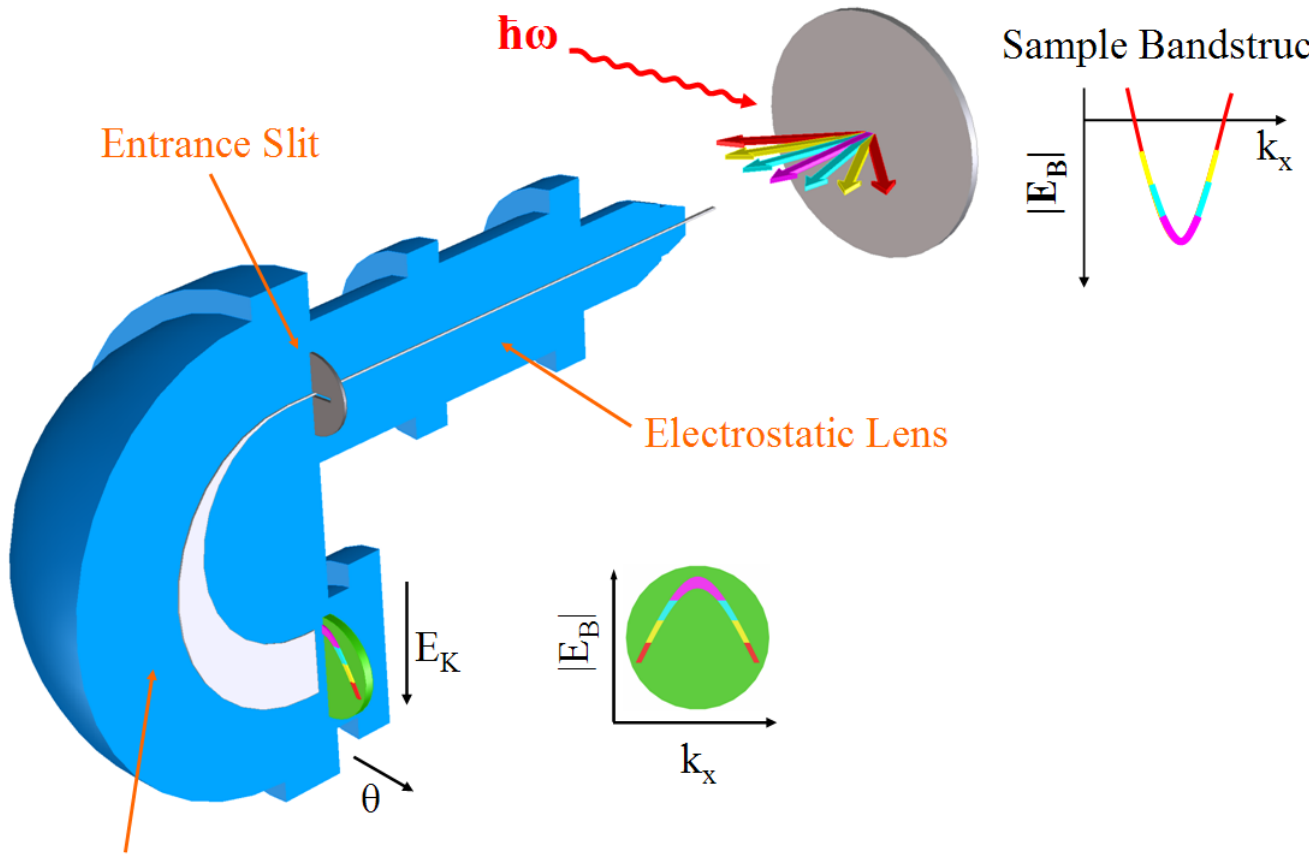

Dispersive Hemisphere

Figure 2.12. A hemispherical analyzer, shown with a 2D imaging detector. Bandstructure along one k-direction can be directly imaged all at once. The 2D detector image corresponds to intensity as a function of $E_{K}$ and angle of emission along the entrance slit direction, $\theta$. This can be converted to an image of intensity as a function of $\left|E_{B}\right|$ and $k$ along the slit direction according to equations 2.8 . 
type of spectrometer can effectively image a window of the $E(k)$ bandstructure along one direction all at once.

The operation of such a spectrometer is shown diagrammed in Figure 2.12. Electrons are photoemitted from the sample distributed in angle and kinetic energy according to the sample's bandstructure. The electrostatic lens column transports the photoelectrons towards the hemispheres retarding/accelerating them to near the pass energy, and images the angles of emission onto the entrance slit. The entrance slit horizontal width represents the analyzers angular acceptance window along the sample's horizontal direction, while the vertical width controls the energy resolution $\left(S_{1}\right.$ in equation 2.38$)$. The electrons then disperse horizontally through the hemisphere according to entrance angle, and vertically/radially according to energy. A circular 2D PSD would record the simultaneous spectrum as shown in the figure. With easily achievable channel densities on the PSD (greater than $100 \times 100$ channels), this type of spectrometer can simultaneously (efficiently) record hundreds of EDCs (and so hundreds of MDCs, as well) at much higher angular and energy resolutions than would be otherwise practical. This type of spectrometer was originally commercialized by the Swedish company Scienta (originally formed from Kai Siegbahn's group) as the "SES-200" 74 and has undergone a number of improved designs with slightly higher performances. Scienta spectrometers since became the ubiquitous spectrometer for photoemission experiments. The spectrometer used with our in-lab system is the Phoibos 150 model from the competing company, SPECS, and offers comparable performance with a slightly smaller size $\left(r_{0}=150 \mathrm{~mm}\right)$.

In practice, ARPES data can be taken filling a 3D phase space volume of $k_{x}$ vs. $k_{y}$ vs. $\left|E_{B}\right|$ very efficiently. As described above, the 2D multichannel detector image corresponds to 2 D planes of data of $\theta$ vs. $E_{K}$ at $\beta$ ( $\theta$ defined as the emission angle along the direction of the analyzer entrance slit, and $\beta$ the angle along the direction perpendicular to the entrance slit in Figure 2.12, and as illustrated if Figure 2.6). After acquiring enough statistics, $\beta$ can be rotated slightly via a sample manipulator, and another data plane of $\theta$ vs. $E_{K}$ can be acquired. This can be continued, as shown in Figure 2.13, until the entire 3D phase space is filled through the desired window. Data in the $\theta$ vs. $\beta$ vs. $E_{K}$ phase space is easily 


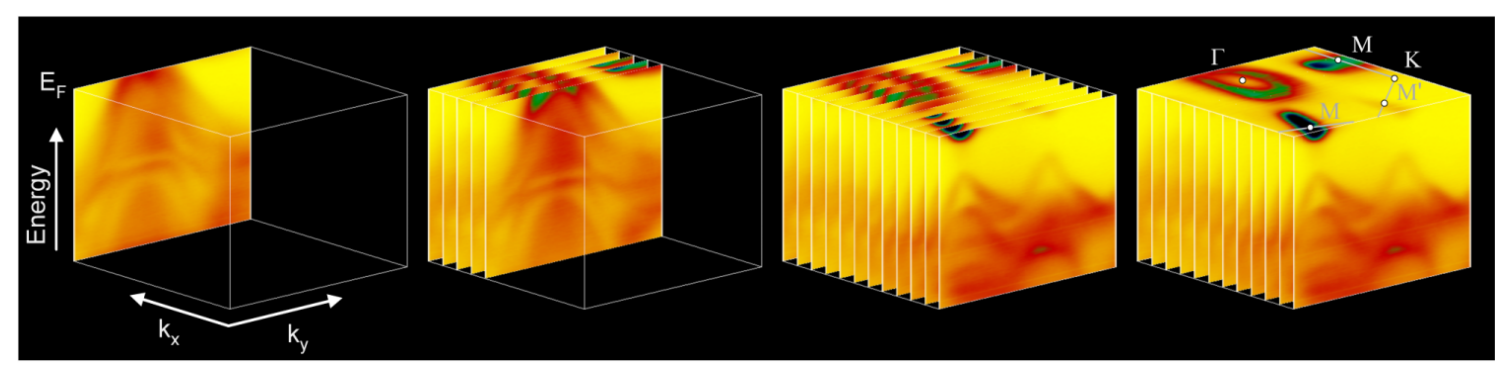

Figure 2.13. ARPES data acquisition in the 3D phase space $k_{x}$ vs. $k_{y}$ vs. energy with a 2D multiplexing SDA, experiment progressing from left to right. The far left shows a single false color plot of ARPES intensity in the 2D plane of $k_{y}$ vs. energy. The entire image is taken simultaneously. Additional 2D planes at different values of $k_{x}$ are taken by rotating the sample orientation with respect to the analyzer entrance slit. This can progress throughout the entire Brillouin zone (or multiple zones even). The far right shows that once the data is taken, it can also be viewed as $2 \mathrm{D}$ planes of $k_{x}$ vs. $k_{y}$ at a given energy. Here the plane at $\mathrm{E}_{F}$, the FS topology, is shown.

converted into $k_{x}$ vs. $k_{y}$ vs. $\left|E_{B}\right|$ following equations 2.8. Viewing the acquired data in $2 \mathrm{D}$ slices of intensity of $k_{x}$ vs. $k_{y}$ at fixed $\left|E_{B}\right|$ illustrates constant energy surfaces; at $\left|E_{B}\right|=0$, the Fermi surface (FS) topology is mapped, as in the far right of Figure 2.13. The high efficiency of these 2D spectrometers allows these FS maps to be taken with unprecedented high energy and angular resolutions, making ARPES a fantastic direct probe of a material's FS, a characteristic central to the understanding of a material's charge dynamics. The data of Figure 2.13 was taken from the layered compound $\mathrm{TiTe}_{2}$ (similar to the first ARPES experiments by Smith, Traum, and DiSalvo ${ }^{49,50,51}$ ) with the in-lab ARPES system using $21.22 \mathrm{eV}$ photons (He lamp) and the Phoibos 150. As this 3 dimensional data set was taken in just over an hour, it is clear that higher dimensional data sets are possible, probing the electronic structure more completely as a function of photon energy, temperature, chemical doping, etc.

The impact that the enormous efficiency (and hence practical resolutions) of the 2D multichannel SDA based spectrometer on the field of ARPES cannot be overstated. One only has to glance at the huge improvement in data quality as the spectrometers advanced to see how entirely new physics is uncovered with high resolution ARPES. Figure 2.14(a) shows an EDC of the $L$-gap $\operatorname{Ag}(111)$ surface state measured at different times during the rapid development of ARPES and spectrometers. The huge efficiency improvements made 
(a) $\mathrm{Ag}(111) \mathrm{L}-\mathrm{Gap}$ Surface State by PES

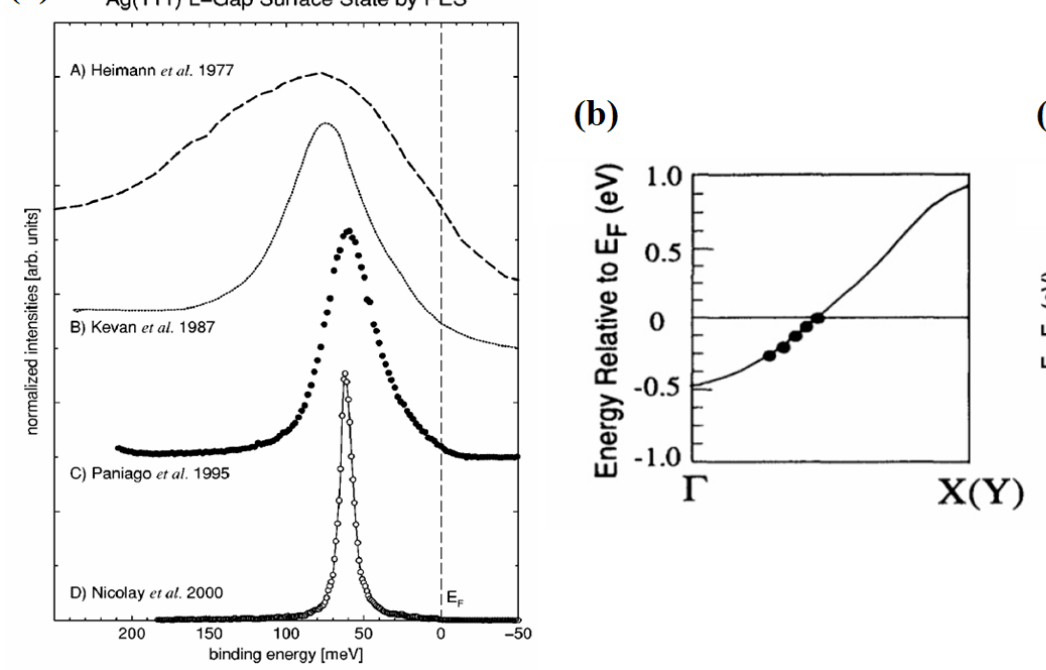

(c)

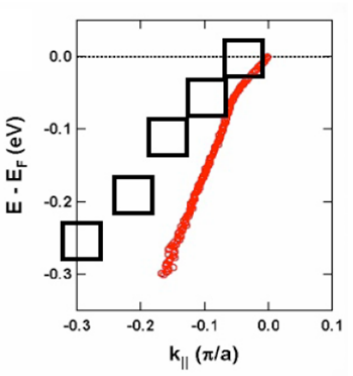

Figure 2.14. (a)ARPES EDCs of the $\operatorname{Ag}(111) L$-gap surface state from several prominent publications over the years. Figure taken from ref. 75. (b)ARPES derived band dispersion of $\mathrm{Bi}_{2} \mathrm{Sr}_{2} \mathrm{CaCu}_{2} \mathrm{O}_{8+\delta}$ taken in 1993 in ref. 76. (c)ARPES derived dispersion taken in 2000 in ref. 77 with a 2D SES-200 spectrometer showing fine features which are not visible in (b). Data points from (b) are overlaid as squares for direct comparison in (c). Panels (b) and (c) taken from ref. 78 .

increasingly higher energy and angular resolutions possible, both of which are required to measure the extremely sharp EDC at the bottom (the bottom spectrum was taken with energy and angular resolutions of $3.5 \mathrm{meV}$ and $\pm 0.15^{\circ}$, respectively). Actually achieving this sharp a feature allows studying physics at a scale that is otherwise impossible. As an example of this, Figure 2.14(b) shows the ARPES EDC derived dispersion from a HTC superconductor taken in 1993 with energy and angular resolutions of $45 \mathrm{meV}$ and $\pm 1^{\circ}$, respectively. Due to low efficiency, only 5 data points along this direction were included. Panel (c) shows a similar data set taken in 2000 using a high efficiency SDA with a 2D multichannel PSD. The increased efficiency allows hundreds of data points to be simultaneously taken along the dispersion with energy and angular resolutions of $14 \mathrm{meV}$ and $\pm 0.1^{\circ}$, respectively. The famous "kink" in the dispersion, and all its related physics, ${ }^{30}$ would clearly never have been observed without these gains in efficiency and resolution. Much of what is presently possible with the ARPES technique is due to the high efficiency of the SDA with 2D multichannel PSD spectrometers. 


\subsubsection{Ultra-high vacuum system}

One of the major requirements of a high-quality photoemission experiment is vacuum. There are three reasons that photoemission experiments must, in general, be performed in a vacuum chamber. The first reason is because of the macroscopic distances $(\approx 1 \mathrm{~m})$ that photoelectrons must travel from the sample to the ultimate detector. The mean free path of free electrons in atmosphere is only about $150 \mathrm{~nm}$, so they will be scattered by gas molecules long before reaching the detector. At pressures around $1 \times 10^{-6}$ torr, the mean free path of the photoelectrons increases to over $100 \mathrm{~m}$, so the vast majority of the emitted electrons will reach the detector without any trouble. The second reason involves modern electron detection devices which are typically "channeltron" or "multichannel plate" (MCP) based detectors. Coincidentally, these detectors often can only be operated in vacuum of around $1 \times 10^{-6}$ torr or better, as well. The third vacuum requirement is by far the most stringent and involves the sample surface.

We saw in section 2.3 that the low inelastic mean free paths of the photoelectrons within most materials is so low that ARPES has a very shallow probing depth. This translates to requiring the sample surface to be high quality with minimal foreign overlayers. If the sample is held in a vacuum of $1 \times 10^{-6}$ torr of gas molecules which have a sticking coefficient of 1 on the sample surface (every molecule which strikes the surface adsorbs, without any subsequent desorption), a full monolayer of the gas will form in only 1 second. After this 1 second, the majority of the ARPES signal will already be from the overlayer rather than the sample itself. Therefore, it is best to have the sample exposed to as low a residual gas pressure as possible, typically well into the ultra-high vacuum range (UHV) with pressures

in the low $10^{-11}$ torr region. This typically requires the entire experiment (sample, sample manipulator, analyzer, excitation source, etc) to be contained within a vacuum chamber system capable of these pressures. In fact, meeting the strict vacuum requirements can form the bulk of the practical work of an ARPES experiment.

Although it is easy to write, UHV is not always so easy to achieve. A pressure of $10^{-11}$ torr is almost 14 orders of magnitude lower pressure than atmosphere, bringing the density 
of gas molecules from $3 \times 10^{19}$ per $\mathrm{cm}^{3}$ down to only $4 \times 10^{5}$. These pressures require the use of high quality stainless steel chambers with all-metal seals/flanges (usually copper gaskets and Conflat flanges). Materials for instrumentation internal to the chamber, or anything else exposed to the vacuum, must be carefully selected to be stable and have minimal vapor pressures both at room temperature and at standard UHV bakeout temperatures (100$200^{\circ} \mathrm{C}$ ). Adequate pumping must also be included and often makes up a large part of the size and expense of an ARPES system. As it can take up to a week to bring a particular chamber from atmospheric pressure down to UHV, it is also extremely beneficial to have an "air-lock" for putting samples into and out of the main vacuum without having to "vent" the entire system. Reliable techniques for moving samples around inside the vacuum system to the analysis position is also a critical dimension to any ARPES experiment.

Just achieving UHV pressures around the sample to be studied is not enough. As the sample was likely exposed to atmosphere prior to insertion into the vacuum system, a clean surface must be somehow freshly prepared in-situ in order to give ARPES data. Different samples can be cleaned with a wide array of recipes. Many surfaces can be prepared with high temperature annealing, or a combination of ion-sputtering and annealing. Other types of crystals can give better surfaces by cleaving the sample in vacuum to expose an entirely fresh surface. Naturally layered materials, such as the HTC cuprates and CMR manganites, have natural cleavage planes which allow this technique to work excellently. Still other types of samples can be "grown" via evaporative techniques, such as molecular-beam epitaxy (MBE), in-situ to give a clean surface for study. In any case, it is often beneficial to have dedicated chambers which can be valved off from the main vacuum for surface cleaning and preparation. Surface characterization tools such as low-energy electron diffraction (LEED) and Auger electron spectroscopy (AES) can often be helpful (in addition to ARPES itself) for determining sample quality and inspecting preparation techniques.

The UHV vacuum system for the lab ARPES station was carefully designed and constructed in order to meet these requirements. Schematics of the system from the 3D modeling software used for design (CoCreate Onespace) are shown in Figure 2.15. The ARPES experiment is performed in the large central chamber. Pumping is provided from the large 


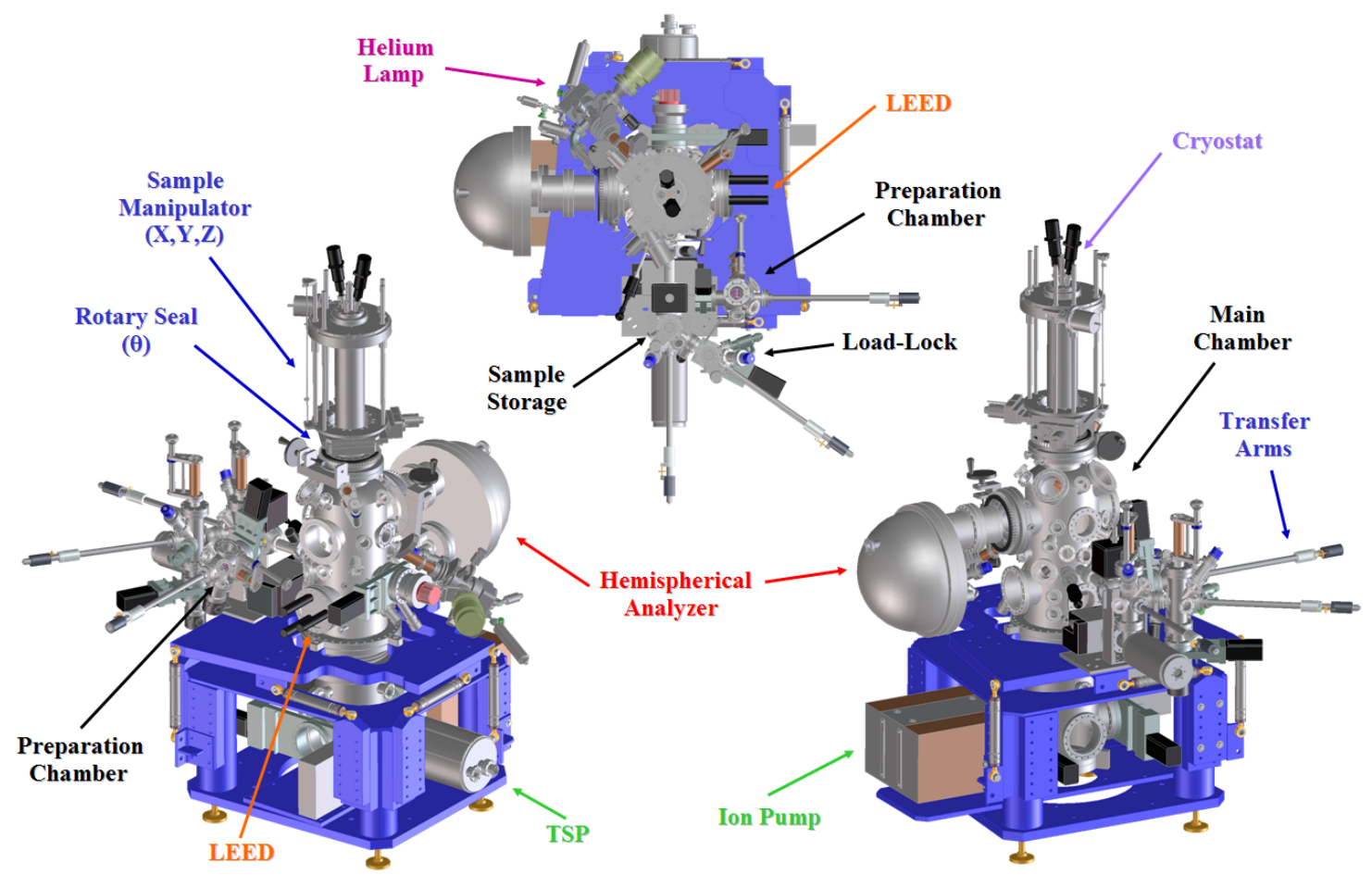

Figure 2.15. 3D schematics of the lab ARPES station. Two side views and a top view.

pumping-tree at the bottom with a large $500 \ell / \mathrm{s}$ ion pump, a $500 \ell / \mathrm{s}$ turbomolecular pump, and a 10" flange titanium sublimation pump (TSP). After a good bakeout, the main chamber has reached as low as $3 \times 10^{-11}$ torr and below $2 \times 10^{-11}$ torr with the additional cryo-pumping when the cryostat is brought to liquid He temperatures. Inserting and removing samples from vacuum is done through a dual-stage air-lock. Up to 12 samples can be inserted/removed directly from atmosphere into the compact "Load-Lock" which can be pumped via a $70 \ell / s$ turbo pump to $\sim 2 \times 10^{-7}$ torr within a half-hour and to $\sim 2 \times 10^{-8}$ torr in a couple hours. Samples can then be individually transfered between this chamber and a sample-storage chamber that is held at $\sim 2 \times 10^{-10}$ torr by an ion pump and a small TSP with the use of a magnetically coupled transfer arm (very similar in concept to that used by Millikan! $\left.{ }^{34}\right)$. Samples are manipulated in vacuum by first affixing them to 1" copper or molybdenum "pucks" which can be and transfered around in the vacuum system in a variety of ways. Sample pucks are held on compact carousels in the load-lock and storage chambers, with the pucks held in place by two angled stiff phosphor bronze wires which 


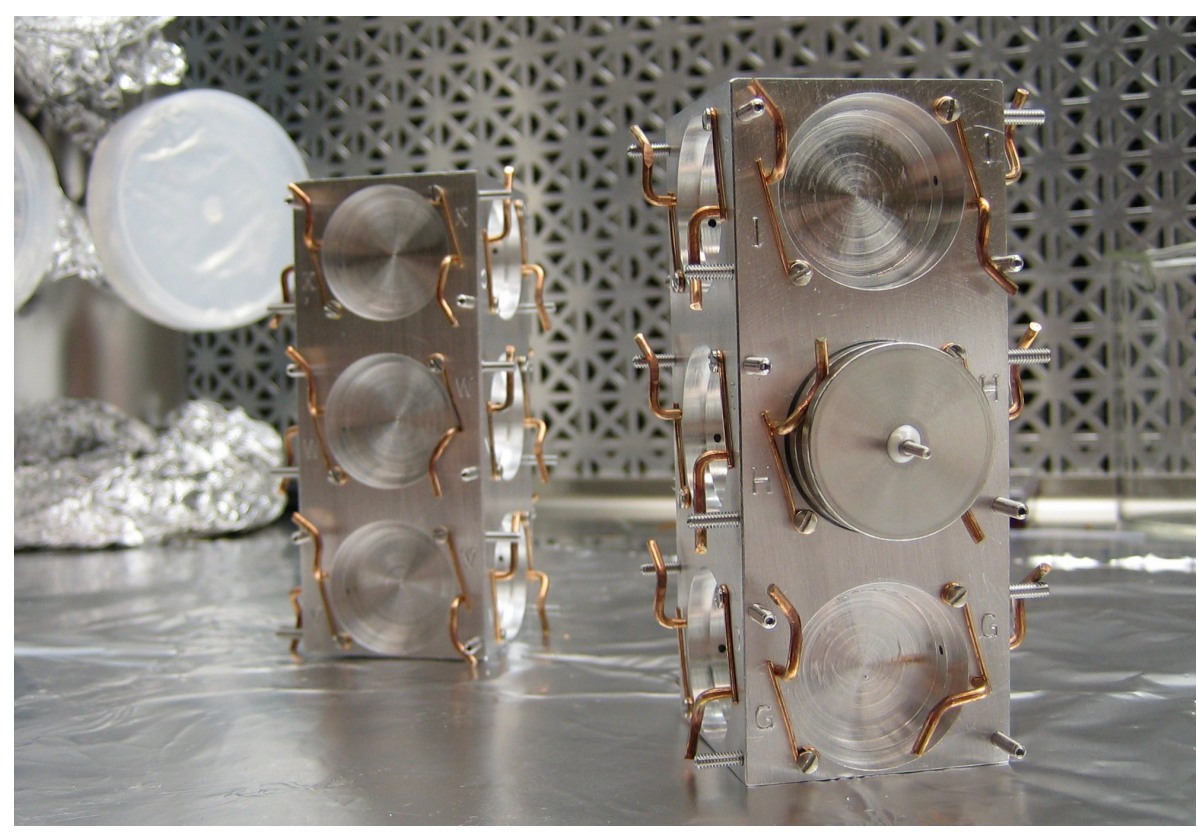

Figure 2.16. Load-lock and sample storage carousels. A stainless steel test puck is shown mounted in a "parking spot", held in place by the two stiff clips. A screw is glued to the surface of this puck where a sample would normally be mounted instead.

clip onto a radial ridge around the edge of the pucks. The carousels before final assembly with a sample puck are shown in Figure 2.16. Samples can be transfered from the storage chamber onto the sample goniometer in the main chamber where ARPES can be taken in addition to AES and LEED. Alternately, samples can be transferred to/from the storage chamber from/to a small chamber dedicated to sample preparation, featuring a custom designed high temperature e-beam sample heating stage and various e-beam evaporators and leak-valves for specific gas species introduction. Due to space constraints which limited the chamber's size, only a small $70 \mathrm{\ell} / \mathrm{s}$ pump is presently used, which achieves a vacuum of only $6 \times 10^{-10}$ torr at best, however, it has still proven to be a useful chamber for sample preparation. For cleaveable samples, a small bellows mounted "wobble-stick" is installed in the main chamber for cleaving samples at low temperature right before the experiment. 


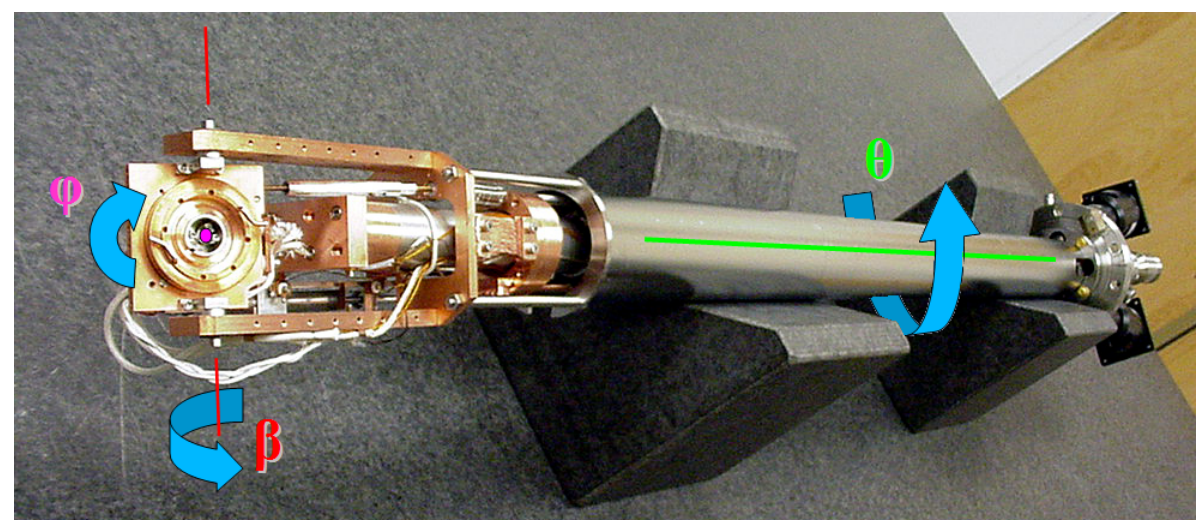

Figure 2.17. Main chamber sample goniometer and cryostat. Goniometer itself provides $\beta$ and $\phi$ rotations. The entire cryostat assembly is mounted on a rotary seal to provide the $\theta$ rotation. Sample stage is connected to tip of cryostat by flexible copper braiding.

\subsubsection{Sample manipulation}

During ARPES or LEED measurement, a sample(puck) is mounted on a 2-axis goniometer at the end of a cryostat designed, built, and maintained by John Pepper and his group at the ALS. Sample pucks are loaded into the goniometer and locked into place in a similar way to scheme used in the Load-Lock and sample storage chambers (Figure 2.16). The goniometer has its own integrated e-beam heater assembly for flash heating samples in the main chamber. It also has two integrated rotational motions: a tilt stage to rotate about the red axis ( $\beta$ in the discussion in section 2.3 and figure 2.6), and a rotational stage to spin about the pink dot $(\phi)$ for azimuthal alignment of the sample's crystallographic axes. The $\theta$ rotation (section 2.3 and figure 2.6) is provided by rotating the entire cryostat/goniometer assembly on a dual-stage differentially pumped rotary seal. The goniometer is held at the end of a vertically mounted liquid He cryostat and thermally connected to the cryotip through a flexible copper braid. This scheme can bring the sample puck to as low as $\sim 12 \mathrm{~K}$. A resistive heater is mounted near the cryotip for regulating temperatures above this base temperature up to room temperature. The copper braid is thermally connected to the cryotip through a sapphire plate which provides good thermal conductivity and electrical isolation such that the sample puck can be floated to high voltage for e-beam heating as well as allowing a measurement of the magnitude of the photoemission current during an 
experiment. In addition to the $\theta$ rotary seal, the cryostat is mounted to the main chamber on a X-Y-Z large bore single-bellows manipulator (see Figure 2.15) enabling large sample translations (to travel the long height differences between the analyzer, LEED, and sample storage locations) in addition to fine sample positioning and alignment during measurement.

\subsubsection{Light source}

Critical to any photoemission experiment is a high quality light source which must meet several requirements. First, the light source must provide photons of energy greater than the sample's work function, or else no electrons can escape into vacuum. Secondly, the bandwidth of the light source is a contributing factor to the total energy resolution of the experiment, so a highly monochromatized source is desired. Third, the experiment count rate will be proportional to the source intensity, and so ARPES greatly benefits from intense sources. Finally, the beamspot should be as small or smaller than the size of the sample or domain of good sample surface to ensure that the measured signal is primarily from the actual sample of interest. Also, the angular resolution of typical analyzers can be adversely affected by large beamspots. The last three requirements taken all together can be described as requiring a source of high "brightness". Due to matrix element effects (section 2.6), it can be very useful to have a tunable source which can have its energy varied over a wide range of energy.

A helium discharge UV lamp (the SPECS UVS300) is installed on the lab system (see Figure 2.15) which can provide both He I and He II lines (21.22 eV and $40.81 \mathrm{eV})$ with good bandwidths of only $\sim 1 \mathrm{meV}$. It is also a fairly intense source and is easy to operate. Unfortunately, however, there are no focusing optics currently. The beam is coupled from the He plasma to the main chamber via a quartz capillary which acts to guide the photons

to the sample as well as limit the amount of helium getting into the main vacuum. The beam is divergent out of the capillary, so with the capillary only a couple cm away from the sample the beamspot is already a few $\mathrm{mm}$ in diameter. This limits its usefulness for small samples, as well as may adversely effect the angular resolution of the electron analyzer. Besides choosing from the two He lines, the source is not additionally tunable (although the 
lamp can be used with other noble gases give a few other photon energy choices). For initial experiments on new types of samples, however, the helium lamp can be very convenient.

The lab chamber system is also capable of being installed on a beamline of synchrotron light sources such as the Advanced Light Source (ALS). Third generation synchrotrons provide extremely bright beams: high intensity $\left(\sim 10^{11}\right.$ photons per second), narrow bandwidth $(\lesssim 10 \mathrm{meV})$, and small spot sizes ( $\lesssim 100$ microns). The most important advantage of a synchrotron undulator beamline for ARPES, however, is the easy continuous tunability of the photon energy, allowing scanning the Brillouin zone along $k_{z}$ for $3 \mathrm{D}$ samples as well as allowing manipulation of matrix elements (section 2.6). The use of modern elliptically polarized undulators (EPU) also allow continuous tuning of the photon polarization, aiding in matrix element adjustment, as well.

An additional characteristic of a light source which is becoming increasingly important is its timing structure. Experimenters are increasingly moving towards performing time resolved experiments ${ }^{79,80,81,82,83,84}$ to probe the dynamics of a sample's electronic structure. A time resolved photoemission experiment could be of a pump-and-probe variety where the light probes the sample at distinct times, adjustably delayed from some repeated pump. This clearly requires a pulsed light source with the length of the probe pulse (as well as the pump) defining the time resolution of the experiment. Energy resolving techniques such as "Time-of-Flight" (TOF) require a pulsed light source, with the achievable energy resolution directly related to the pulse width of the source. The TOF technique is central to the work of this thesis and will be discussed in detail in the following chapter. A helium discharge lamp is continuous and so is not an option for these techniques. Sychrotrons such as the ALS usually operate in "quasi-continuous" mode in which the light does arrive in well defined pulses, but with such a high repetition rate that they cannot be used for timing experiments. However, regularly scheduled alternate operating modes lower the repetition rate such that timing experiments become feasible. Laser sources (with harmonic generation to increase photon energy to usable levels) have become particularly attractive for photoemission experiments due to their intrinsic short pulses. Towards these ends, a 


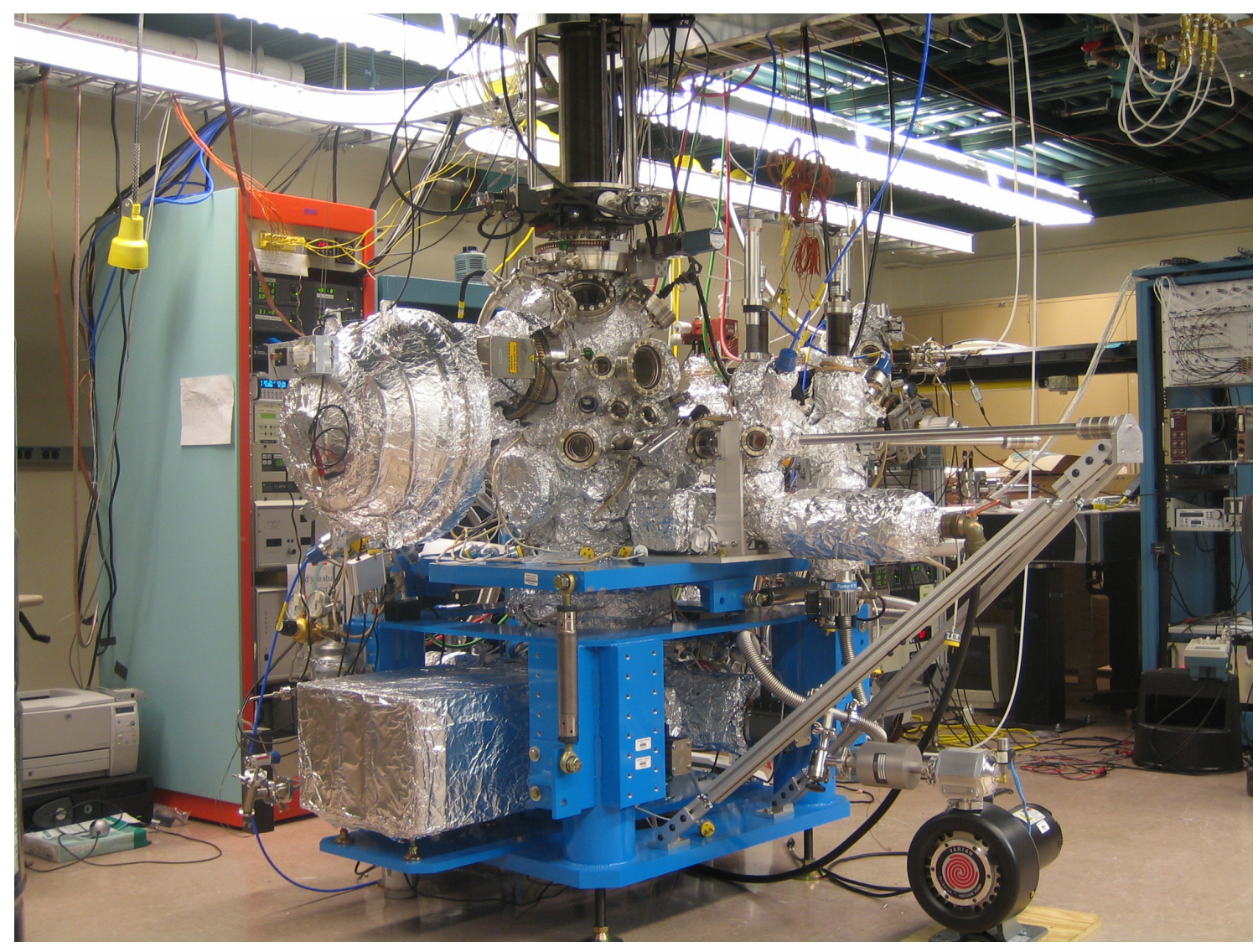

Figure 2.18. Photograph of the fully constructed and operational ARPES system. The analyzer is on the left-hand side.

fourth-harmonic $6 \mathrm{eV}$ laser light source was developed in the lab for use with conventional photoemission analyzers, but has also become a key component of the spin-TOF project.

A photo of the custom designed and fully operational in-lab ARPES station is shown in Figure 2.18. 


\section{Chapter 3}

\section{Spin-Resolved Photoemission Spectroscopy}

Further and unique insight into the electronic structure of solids and into the photoemission process can be obtained by analyzing the spin orientation of the emitted electrons

- R. Feder ${ }^{85}$

The previous chapter gave a brief look into the power of ARPES for probing the electronic structure of solids. It was shown that by resolving both photoelectron energy and momentum (emission angle), high-resolution ARPES can not only perform bandmapping and Fermi surface topology mapping, but can also provide information regarding many-body interactions through direct access to the single particle spectral function. This chapter will detail how resolving the spin orientation of the photoelectrons can provide additional information about a material's electronic structure as well as insights into the photoemission process itself. Spin polarization (degree of imbalance between spin-up and -down along some axis) of photoelectrons is certainly expected from ferromagnetic systems in which the density of states is polarized, but photoemission from non-magnetic samples may also be spin polarized due to the spin-orbit interaction and the use of circularly polarized light. Either way, additional information can be obtained with measurement of the photoelectron 
spin. These cases will be explored and the current state-of-the-art methods for performing spin-resolved photoemission will be reviewed. Further details are covered in numerous spin-resolved photoemission reviews. ${ }^{16,22,23,24}$

\subsection{Three-step model revisited}

As discussed in section 2.3, the most realistic formulation of photoemission treats it as a single coherent one-step process. A spin-dependent treatment is no different in this respect, and a general one-step approach should be considered for maximum accuracy in

numerical calculations. ${ }^{85}$ However, just as in the spin-independent case, use of the simpler three-step model provides a more intuitive framework for understanding and interpretation. In the spin-dependent case, the three-step model also provides better insight to the various sources of spin polarization in the photoemitted electrons.

\subsubsection{Step one}

The first step is the actual photoexcitation process in the solid and contains the information intrinsic to the electronic structure being probed. The rate of these direct transitions can be approximately expressed as a "Fermi's Golden rule" as in equation 2.13, which was reduced to equation 2.22 in section 2.4 to highlight the relevance of the single particle spectral function. We can copy this equation here, explicitly noting the spin-dependence which was earlier ignored, as

$$
I(\mathbf{k}, \omega, \sigma) \propto \sum_{f, i, \sigma_{i}}\left|\left\langle\phi_{f}^{\mathbf{k}, \sigma}\left|H_{r a d}\right| \phi_{i}^{\mathbf{k}, \sigma_{i}}\right\rangle\right|^{2} f(\omega) A(\mathbf{k}, \omega, \sigma) \delta\left(\omega+E_{K}-h \nu\right)
$$

where $\sigma$ is the spin direction (i.e. spin-up or -down along some axis) of the photoelectron, $\sigma_{i}$ is the spin direction of the initial state, and we have written out the matrix elements, $\mathcal{M}_{f, i}^{\mathbf{k}}$. We can now see several reasons to expect spin-dependence of the photoelectron intensity. We expect that ferromagnetic materials will have a strongly spin-dependent electronic structure; spin-dependence of $\left|\phi_{i}^{\mathbf{k}, \sigma_{i}}\right\rangle$ will be passed on to the photoemitted electrons through the matrix element term. We would also expect the spin dependent electronic 
structures of ferromagnets to have a spectral response to the removal of an electron dependent on its spin. This is expressed as the spin-dependence of the spectral function term, $A(\mathbf{k}, \omega, \sigma)=\sum_{m}\left|\left\langle\Psi_{m}^{N-1}\left|c_{\mathbf{k}, \sigma}\right| \Psi_{i}^{N}\right\rangle\right|^{2} \delta\left(\omega-E_{m}^{N-1}+E_{i}^{N}\right)$. Spin-polarization can also arise in non-ferromagnetic systems from the matrix element term in equation 3.1 through dipole selection rules.

The matrix elements were investigated using a spinless Hamiltonian of Schrödinger form in section 2.6. Here we begin with a proper Dirac form which takes the electron spin into account: ${ }^{24}$

$$
H=\frac{1}{2 m_{e}}\left(\mathbf{p}-\frac{e}{c} \mathbf{A}\right)^{2}+e \phi-\frac{e \hbar}{2 m_{e} c} \boldsymbol{\sigma} \nabla \times \mathbf{A}+\frac{i e \hbar}{4 m_{e}^{2} c^{2}} \mathbf{E} \cdot \mathbf{p}-\frac{e \hbar}{4 m_{e}^{2} c^{2}} \boldsymbol{\sigma}(\mathbf{E} \times \mathbf{p})
$$

where $\boldsymbol{\sigma}$ is the vector of Pauli matrices used to represent the electron spin (using the notation from section 1.2 we have $\left.\mathbf{S}=\frac{\hbar}{2} \boldsymbol{\sigma}\right)$. The first two terms were used previously in section 2.6, and the fourth term is a relativistic energy correction, which we can ignore here. The first two terms can be again reduced to the electric dipole $\mathbf{A} \cdot \mathbf{p}$ perturbation term as in section 2.6. Electric dipole transitions are spin-conserving; $\left\langle\phi_{f}^{\mathbf{k}, \sigma}|\mathbf{A} \cdot \mathbf{p}| \phi_{i}^{\mathbf{k}, \sigma_{i}}\right\rangle \neq 0$ only when $\sigma=\sigma_{i}$.

The third term, which can be written as $-\frac{e \hbar}{2 m_{e} c} \boldsymbol{\sigma} \cdot \mathbf{B}$, represents the direct coupling between the spin and the magnetic field. Photoexcitation through this term (where $\mathbf{B}$ is the magnetic field of the incident radiation) leads to spin-flip transitions where the electron spin is switched. In fact, with circularly polarized light of positive helicity, only initial state electrons with spin-down (along the light propagation direction) can be excited and get flipped to spin-up final state photoelectrons, and vice versa for light of negative helicity. ${ }^{85}$ This seems to lead to $100 \%$ spin polarized photoemission even from non-magnetic samples. The cross section for these magnetic transitions to occur, however, need to be compared with the other terms of equation 3.2. An early calculation ${ }^{86}$ estimates that the ratio of spin-flip $\boldsymbol{\sigma} \cdot \mathbf{B}$ transitions to spin-conserving $\mathbf{A} \cdot \mathbf{p}$ transitions go as $\approx h \nu /\left(m_{e} c^{2}\right)$. At $h \nu=100 \mathrm{eV}$, this means spin-flip transitions will be only $0.02 \%$ as likely as spin-conserving ones. This ratio can increase in geometries where the $\mathbf{A} \cdot \mathbf{p}$ matrix elements vanish (section 2.6), however the $\boldsymbol{\sigma} \cdot \mathbf{B}$ matrix elements will still be quite small resulting in little total photoemission to practically measure. A somewhat intuitive explanation for the relatively small probability 
of $\boldsymbol{\sigma} \cdot \mathbf{B}$ transitions can be found by multipole expansion: in the absence of strong spin-orbit splitting, the magnetic dipole (M1) excitations are forbidden (due to parity), and so the strongest contribution will be from only quadrapole (M2) or higher order terms. ${ }^{85}$ Due to the domination of the $\mathbf{A} \cdot \mathbf{p}$ over the $\boldsymbol{\sigma} \cdot \mathbf{B}$ term, we can treat the photoexcitation step as a spin-conserving process.

The last term in equation 3.2 represents spin-orbit coupling, introduced in section 1.2.3, and can have considerable effects in determining the initial and final states. These can lead to measurable and even large spin-polarization effects in photoelectrons through the dominant electric dipole transition term. The mechanism which can make this occur is better explained in section 3.2 .

We have found, then, that the matrix element in equation 3.1 can be be well approximated as

$$
\left\langle\phi_{f}^{\mathbf{k}, \sigma}\left|H_{r a d}\right| \phi_{i}^{\mathbf{k}, \sigma_{i}}\right\rangle \propto\left\langle\phi_{f}^{\mathbf{k}, \sigma}|\mathbf{A} \cdot \mathbf{p}| \phi_{i}^{\mathbf{k}, \sigma_{i}}\right\rangle
$$

which can be reduced to the same electric dipole matrix element as arrived at in section 2.6, except with explicit spin dependence included in the initial and final states. As discussed above, these transitions are spin-conserving; non-zero matrix elements can only occur for $\sigma=\sigma_{i}$, so we can drop the $i$ notation and remove the sum over $\sigma_{i}$ in equation 3.1. So after all our work, we find that the spin-dependence of the photoexcitation intensity is just

$$
I(\mathbf{k}, \omega, \sigma) \propto \sum_{f, i}\left|\left\langle\phi_{f}^{\mathbf{k}, \sigma}|\mathbf{A} \cdot \mathbf{p}| \phi_{i}^{\mathbf{k}, \sigma}\right\rangle\right|^{2} f(\omega) A(\mathbf{k}, \omega, \sigma) \delta\left(\omega+E_{K}-h \nu\right) .
$$

\subsubsection{Steps two \& three}

Photoexcited electrons from step one must still travel through the solid and transmit through the surface before we are able to measure them. A natural concern is the degree to which spin polarization of the photoelectrons is maintained through steps two and three.

For non-magnetic materials, the mean free paths of electrons tend not to be spin dependent, and so if photoelectrons from step one are not spin-polarized they will not gain spin polarization through scattering in the bulk. In certain non-magnetic semiconductors, like GaAs, the conduction band can be populated with spin-polarized carriers from excitation 
by circularly polarized light, and thermal diffusion of these charge carriers becomes a dominant transport mechanism. A spin-lifetime, which is the average time before a spin-flip scattering event occurs, can be introduced. It can be shown that if the spin-lifetime is much shorter than the regular photoelectron lifetime, depolarization of these carriers can occur. ${ }^{85}$

For magnetic materials, spin-dependent scattering in step two can lead to interesting effects. It is generally found that the low kinetic energy secondary electrons (large increasing tail of measured spectrum in Figure 2.4) can have considerable polarization, even greater than that of the valence band. ${ }^{87}$ This is generally explained qualitatively with a basic phase space arguments. ${ }^{22}$ For a photoelectron to flip its spin by scattering via the Coulomb interaction (total spin-conserving), an electron from an occupied state must also flip its spin and scatter to an available state above $\mathrm{E}_{F}$. The ferromagnetism of the sample typically means there is a larger number of minority spin states above $\mathrm{E}_{F}$ than majority states. So for spin flip scatterings, it is easier for an occupied majority electron to find an available minority state above $\mathrm{E}_{F}$, making it more likely for a spin minority photoelectron to flip to majority than vice versa. This leads to an interesting situation where the highest spin polarization of the photoemitted spectrum is at the kinetic energies where there is maximum intensity (note the height of the secondary peak in Figure 2.4). This effect has been taken advantage of in making polarized secondary electron scanning microscopes (or scanning electron microscope with polarization analysis, SEMPA) for high resolution magnetic domain microscopy. ${ }^{88}$

Despite the various possibilities for loss of spin-information in transport and transmission through the surface, it has been experimentally found in many cases that spin polarization of primary photoelectrons is not significantly altered in the emission process, allowing straightforward interpretation of the experiment. In other cases things can be less clear, although even in these cases, the loss of spin-polarization can be used for understanding the energy and spin dependent scattering of excited electrons. ${ }^{89}$ 


\subsection{Spin resolved PES from non-magnetic samples}

Using circularly polarized light to photoemit electrons with preferential spin orientation from a non-polarized source was first suggested in 1969 by Fano with unpolarized Cs atoms ${ }^{90}$ and experimentally verified in 1970 by Kessler and Lorenz. ${ }^{91}$ The polarizing effect is due to spin-orbit coupling in the initial and/or final states and the selection rules enforced in the dipole matrix element in equation 3.4. A simple rationale for this can be understood as follows: the angular momentum of the photon is linked to the electron initial and final state (photoelectron) orbital quantum numbers $\left(l, m_{l}\right)$ through the dipole selection rules of the matrix element in equation 3.4, and these angular momenta are in turn coupled to the electron spin through the spin-orbit coupling in equation 1.22. Circularly polarized photons have absolute angular momentum defined along their propagation direction, and thus information regarding the orbital momentum of the probed states can become encoded in the photoelectron spin polarization along this axis. This concept is perhaps best illustrated in an example of great significance in the field of spin physics as it has become a popular standard source for spin polarized electron beams for various experiments: the GaAs photocathode. ${ }^{92,93,94}$

GaAs is a direct-gap semiconductor with its bandstructure around $\Gamma$ sketched at the far left of Figure 3.1. At the high symmetry points at $\Gamma$, the states' symmetries can be closely approximated by those of atomic orbitals. The bottom of the conduction band at $\Gamma_{6}$ can be represented as a $s_{1 / 2}$ state. The valence band can be approximated by a $p$ level, which by strong spin-orbit coupling (section 1.2.3) is split by $\Delta$ into two $p_{3 / 2}$ states (degenerate at $\Gamma$ ) and a lower $p_{1 / 2}$ state. GaAs is not magnetic, and so each band shown is additionally doubly spin-degenerate.

The right side of Figure 3.1 is a level diagram of each state in the bands at $\Gamma$. Each state is labeled on top by the $m_{j}$ value according to the total angular momentum $\left|j, m_{j}, l, s\right\rangle$ eigenbasis. Each $\left|j, m_{j}\right\rangle$ state is also written out below in terms of the $\left|l, m_{l}, s, m_{s}\right\rangle$ eigenbasis, here abbreviated as $\left|m_{l}, m_{s}\right\rangle$ kets. Excitation by circularly polarized light must follow the regular selection rules of $\Delta l= \pm 1, \Delta j= \pm 1,0$, and $\Delta m_{j}= \pm 1\left(m_{j}\right.$ directed 

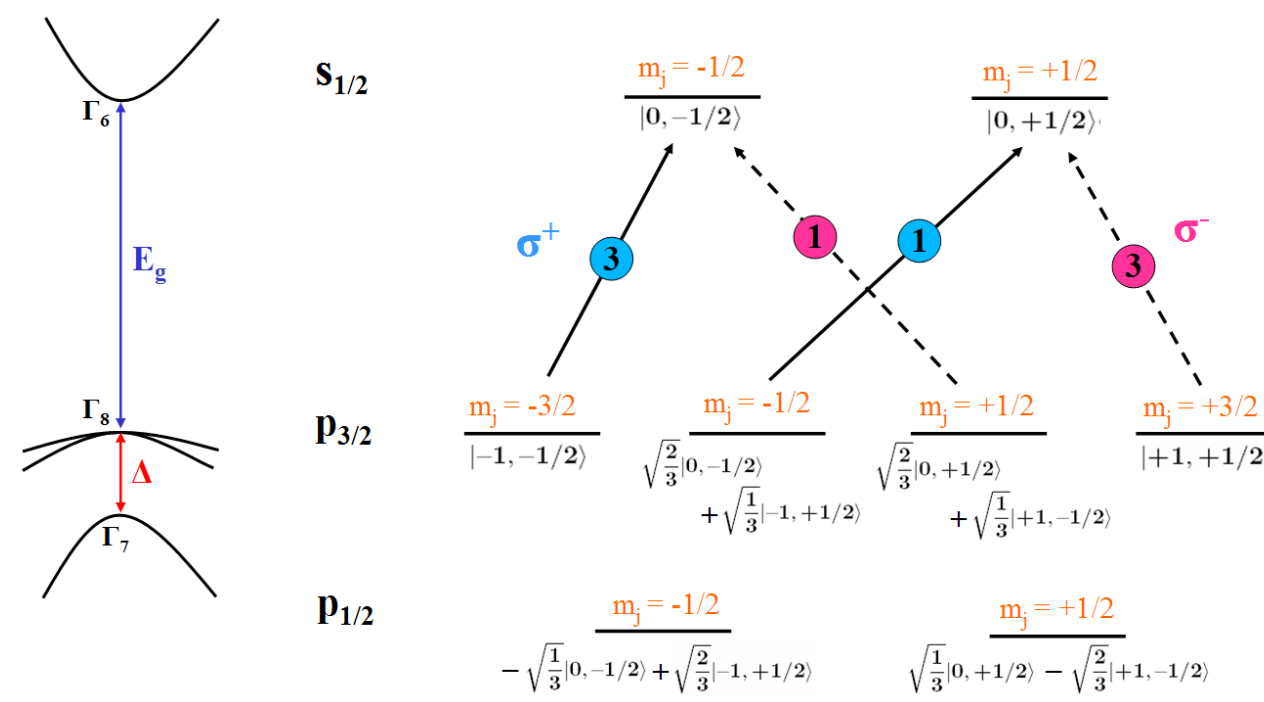

Figure 3.1. Diagram of optical excitation of GaAs at $\Gamma$ with circularly polarized light. Schematic bandstructure at $\Gamma$ is shown on far left, labeling high symmetry points. Atomiclike spectral notation for the three levels at $\Gamma$ are given. Complete level diagram of each $\left|j, m_{j}\right\rangle$ level, expanded into explicit $\left|m_{l}, m_{s}\right\rangle$ basis kets is on the far right. Right circularly polarized light of $h \nu=E_{g}$ can only make transitions labeled by the solid arrows. The labels ' 3 ' and ' 1 ' correspond to the transitions' relative strengths.

along the photon propagation direction). Illuminated by right circularly polarized light of $h \nu=E_{g} \sim 1.5 \mathrm{eV}$, transitions are then restricted by the last selection rule to only the two marked by the solid arrows in the figure. The relative transition rates can be worked out by inspecting the dipole matrix elements $\left(\left|\left\langle m_{j}^{\prime}|\mathbf{A} \cdot \mathbf{p}| m_{j}\right\rangle\right|^{2}\right)$ in terms of the $\left|m_{l}, m_{s}\right\rangle$ basis. As the dipole operator conserves spin, it is clear that the relative transition rate of the $m_{j}=-3 / 2 \longrightarrow m_{j}=-1 / 2$ transition to the $m_{j}=-1 / 2 \longrightarrow m_{j}=+1 / 2$ transition is 3 to 1 . It is interesting to note how the transition rates are ruled by the Clebsch-Gordon coefficients. Because of this imbalance in transition rates, the photocurrent obtains an imbalance of spin-up and -down electrons. To be precise, the final state polarization (along the photon propagation direction) can be expressed as

$$
P=\frac{N_{\uparrow}-N_{\downarrow}}{N_{\uparrow}+N_{\downarrow}}=\frac{1-3}{1+3}=-50 \%
$$

Note that illumination by left circularly polarized light limits the transitions to the two marked by the dashed arrows in the figure, which will then give the exact opposite spin polarization of $+50 \%$. 
This example well illustrates that the spin-orbit splitting of the valence band is critical for the effect. If there were no spin-orbit splitting, the $p_{1 / 2}$ levels at $\Gamma_{7}$ would also be able to participate in overlapping transitions. A similar analysis to above shows that with right circularly polarized light, the additional $m_{j}=-1 / 2 \rightarrow m_{j}=+1 / 2$ transition would occur at a rate of 2 relative to the others. This would result in a total excited spin ratio of 3 to 3 ; in other words, no spin polarization. Equally critical is the use of circularly polarized light. Inspection can show that linearly polarized light, with its different selection rules, will not result in spin polarization (this is perhaps easiest to see by thinking of the linear polarized light as a superposition of both left and right circular light). This is actually a firm rule for bulk transitions: excitation by linear polarization will not create spin polarization, but circular/elliptical may and reversal of the helicity reverses the polarization effect. ${ }^{85}$ It should be noted that this example and rule explicitly involves only photoemission normal to the surface; in general, at emission angles away from normal, spin-orbit splitting can lead to polarized emission even with linear or unpolarized light. ${ }^{95}$ We will see an example of this in section 6.2.3.

The present effect with circularly polarized light is sometimes referred to as "optical spin orientation" and is more thoroughly described in ref. 89. More exhaustive group theoretical approaches to the symmetry of the bands, including away from the $\Gamma$ point, can describe the effect more completely. By measuring the spin polarization of photoemission from samples with strong spin-orbit coupling, one can thus work backwards to experimentally map the symmetries of the probed bandstructure. Examples of this, in particular the resulting symmetry-resolved bandmapping of Pt, are well reviewed in ref. 96. Other good examples of using this technique to investigate spin-orbit splitting in the valence bands of non-magnetic $3 \mathrm{~d}$ transition metals can be found in ref. 97.

An extremely interesting, although difficult to interpret, spin-resolved photoemission experiment has also been performed on the HTC superconducting cuprates. ${ }^{21}$ These HTC's are of course not ferromagnetic - in fact they have an antiferromagnetic background. Averaged over the many unit cells within a reasonable illumination spot, there will be no spin polarization in the initial states. However, again through the use of circularly polarized light 


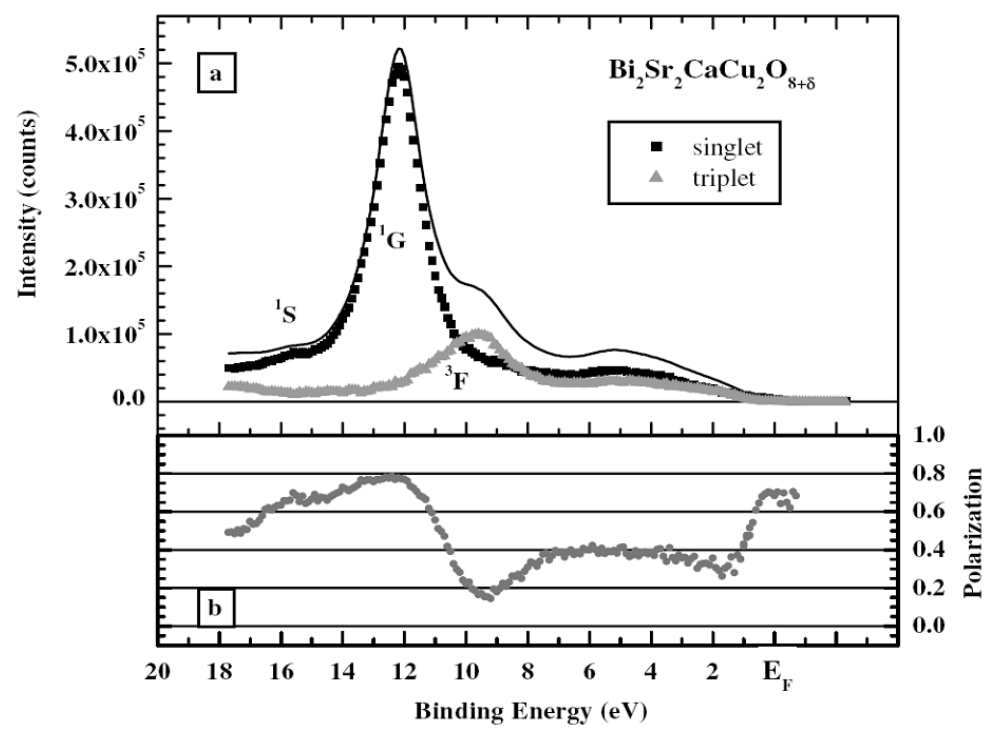

Figure 3.2. Spin-resolved resonant photoemission spectra from $\mathrm{Bi}_{2} \mathrm{Sr}_{2} \mathrm{CaCu}_{2} \mathrm{O}_{8+\delta}$ at photon energy $h \nu=931.1 \mathrm{eV}$, the $\mathrm{Cu} 2 \mathrm{p}_{3 / 2}$ absorption edge. (a)The total intensity spectrum is the solid line, and the spectrum broken up into spin-triplet and -singlet components are the square and triangle markers, respectively. (b) The measured spin polarization of the resonant photoemission, used for the analysis of the singlet and triplet components. Figure taken from ref. 21.

and a fairly large spin-orbit splitting of the $\mathrm{Cu} 2 \mathrm{p}$ core levels, spin polarized photoemission is achieved. Analysis of the spin polarization then offers insight into the spin structure of the initial state, in this case the spin-singlet and -triplet character of the spectrum, which has impact on the typical starting point for theoretical models of the cuprate electronic structure. Full interpretation involves a thorough analysis of the resonant photoemission used to take advantage of the large spin-orbit splitting of the $2 \mathrm{p}$ levels, which is beyond the scope of the thesis. ${ }^{98,99}$ A complete description of the experiment can be found in the literature. ${ }^{19,20,21}$

A brief look at the experimental results, however, is instructive and they are presented in Figure 3.2. The total spin integrated EDC spectrum is shown by the solid line in panel (a). The measured spin polarization resulting from the use of circularly polarized light and the spin-orbit split core level is shown in panel (b). Through a fairly complicated interpretation and analysis, ${ }^{20}$ this polarization measurement is used to break down the solid line spectrum in (a) into spin-singlet and -triplet components, shown by the square and triangle markers. 
It should be stressed how interesting it is that spin information is being obtained from an unpolarized source. An important aspect of the data to notice is the resolution: only one EDC was taken and it was taken with essentially no angular resolution (averaged over $40^{\circ}$ ) and only $750 \mathrm{meV}$ energy resolution. Although it is quite an important work, the resolutions are quite a bit worse than the ultra-high resolution regular ARPES is capable of (section 2.7.1) and is providing no access to the momentum dependence which is critical for complete understanding of the solid. We will investigate why this is so in section 3.4.

\subsection{Spin resolved PES from ferromagnets}

Spin-resolved photoemission can also be applied to great use on ferromagnetic materials. Ferromagnetism is due to an imbalance of the distribution of spin-up and -down electrons in a material's electronic structure, with the magnetization vector providing the natural reference axis for spin orientation. This imbalance can be directly probed via spin-resolved photoemission as the polarization of the measured photoelectrons is directly related to the polarization before excitation with unpolarized or linearly polarized light, as detailed in section 3.1. Initial work was hampered by requiring the sample to be placed in a constant applied magnetic field to ensure alignment of ferromagnetic domains, but working with samples which can be remnantly magnetized (and shaped to have little stray field to disrupt the photoelectrons) allows ARPES experiments to be properly performed. ${ }^{22}$ In this way, spin-ARPES can directly map the majority/minority spin-resolved bandstructure and Fermi surface topologies of bulk and surface states of ferromagnets. Thus spin-ARPES can be a key tool in engineering advanced spintronics-type materials, interfaces, and devices, as well as provide excellent opportunities for comparison with advanced theories of ferromagnetism in general. Specifically, near $\mathrm{T}_{C}$ models of ferromagnetism are still in doubt, and temperature dependent spin-ARPES is a direct way to probe the ferromagnetic transition. ${ }^{22}$ Detailed spin-ARPES experiments also allow access to the spin-dependent single-particle spectral functions which offers a way to measure the spin-dependent self- energies and correlation effects. 

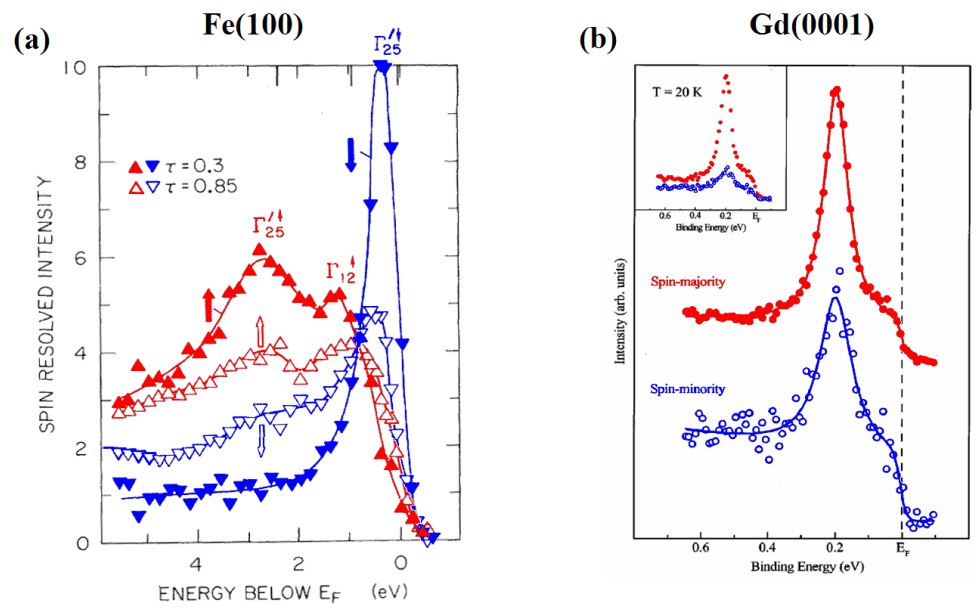

Figure 3.3. (a) Spin-resolved ARPES normal emission EDC's from Fe(100) taken with spolarized photons with $h \nu=60 \mathrm{eV}$. Up (red) and down (blue) filled arrows are majority and minority signal, respectively, at $\mathrm{T}=0.3 \mathrm{~T}_{C}$. Open triangles are at $\mathrm{T}=0.85 \mathrm{~T}_{C}$. Figure adapted from ref. 100. (b) Spin-resolved ARPES from Gd(0001)/Mo(110) at 20K with photon energy $h \nu=21.2 \mathrm{eV}$. Solid (red) and open (blue) markers are spin majority and minority, respectively. Solid lines are Lorentzian fits. Inset shows the relative intensity of the two signals. Figure adapted from ref. 101.

One example of such work is shown in Figure 3.3(a) where normal emission spin-resolved spectra from $\mathrm{Fe}(100)$ are shown. ${ }^{100}$ The spectra taken well below $\mathrm{T}_{C}$ (solid markers) match theory quite well. The spin majority EDC shows two clear peaks corresponding to the $\Gamma_{25}$ and $\Gamma_{12}$ majority bands. The spin minority EDC shows a sharp peak near $\mathrm{E}_{F}$ corresponding to the $\Gamma_{25}$ minority band: the difference between the two $\Gamma_{25}$ peaks is, of course, the exchange splitting. Well above $\mathrm{T}_{C}$ when the $\mathrm{Fe}$ is no longer ferromagnetic, one would expect there to be no spin-polarization, and that the majority and minority EDC's would be identical. The interesting thing to study is how the below $\mathrm{T}_{C}$ spectra evolves into the above $\mathrm{T}_{C}$ spectra as $\mathrm{T}$ is increased. The open markers display the majority and minority bands at $\mathrm{T}=0.85 \mathrm{~T}_{C}$, and quite a lot of things can be noted. First of all, although there is a definite decrease in overall intensity in both spin channels, the peaks present in the far below $\mathrm{T}_{C}$ data have not shifted in energy very much in the high temperature data. The Stoner model of ferromagnetism would predict that the exchange splitting should collapse as $\mathrm{T}_{C}$ is approached and the overall magnetization decreases, and this is certainly not occurring here. Second, a new peak appears in the minority EDC, very near the energy of 
the majority $\Gamma_{25}$ peak. This is interpreted as an "extraordinary peak" and is evidence of regions of the sample where the magnetization direction spontaneously fluctuates, effectively mixing the signal in the two spin channels. A more thorough review can be found in ref. 22. This data represents great progress in the understanding of ferromagnetism near $\mathrm{T}_{C}$ in Fe. However, again we should take note of the resolutions used in the experiment: $300 \mathrm{meV}$ and $\pm 3^{\circ}$ energy and angular resolution, respectively. For this particular system where the high temperatures likely do not allow benefit of higher resolutions, it is exciting to think what other details could be lurking at finer scales for other ferromagnetic systems near $\mathrm{T}_{C}$ (for an example, see section 7.2.1).

A second example is shown in Figure 3.3(b) which displays spin-ARPES spectra from the $\operatorname{Gd}(0001)$ surface. ${ }^{101}$ A relatively sharp peak is found in both the majority and minority EDC's, although the minority peak is much less intense, as shown in the inset. This experiment was performed with $50 \mathrm{meV}$ and $\pm 3^{\circ}$ energy and angular resolutions. As the band being probed has very slow dispersion, the angular integration does not increase the peak widths very much. These resolutions represent about the best spin-ARPES resolutions in the present literature. Due to the higher resolutions, the peaks can be fairly well fit by Lorentzians, as shown in the figure, and widths can be accurately extracted. In this case the spin minority peak is found to be significantly wider than the majority, and the dependence of these widths on temperature aid in studying the many-body effects in the sample. ${ }^{101}$ This is quite an accomplishment for spin-ARPES, however the resolutions still are not as good as those obtained with regular ARPES (such as in Figure 2.14) and was partly able to resolve the peaks so well only because good angular resolution was not required for the flat band being probed.

\subsection{Current state-of-the-art technique}

Spin-resolved photoemission experiments have been performed for almost as long as spin-integrated experiments. Spin-polarization was first detected in photoemission in 1969 as a "total-yield" experiment ${ }^{102}$ (i.e. all electrons measured, no angle or energy resolution). 
The first spin, angle, and energy resolved experiments were published in 1981 in both magnetic ${ }^{103}$ and nonmagnetic ${ }^{104}$ systems. Despite the early start, it is clear that spinARPES experiments have not experienced the same explosion of advances which regular ARPES has enjoyed. We saw in section 2.7.1 that much of the reason for the enormous ARPES growth is due to the significant advances in instrumental efficiency. We shall show here that spin-ARPES is severely handcuffed by significantly lower efficiencies, hampering the development of instrumentation to keep up with the field of spin-integrated ARPES.

The primary problem can be summarized in a single sentence: measuring electron spin orientation is extremely difficult! Light polarization analysis is straightforward; optical polarization filters which pass close to $100 \%$ of a given polarization while rejecting close to $100 \%$ of the orthogonal polarization are commonplace. Unfortunately, no such device exists for electron spin. All detectors so far developed for electron spin polarization analysis, or electron polarimeters, are based on some sort of spin-dependent electron scattering method, which always turn out to be quite inefficient compared to the optical case. The state-of-theart polarimeters can achieve under good conditions an effective efficiency of $1 \times 10^{-4}$ ! Worse yet, they are only single-channel detectors.

The net result of these two critical points are summarized in Figure 3.4. Because of their familiarity and enormous effectiveness, SDA's are now usually used to perform the energy and angle analysis in a spin-ARPES instrument. ${ }^{105,106,107,108}$ As shown in the figure and discussed in section 2.7.1, a multichannel PSD can be used in regular ARPES to detect a given count rate in over $10^{4}$ separate channels simultaneously. This allows ARPES data to be taken as $2 \mathrm{D}$ slices as depicted to the right of the figure. In a spin-ARPES instrument, the spin-integrating PSD is replaced by a single-channel spin polarimeter. Not only is this single channel on the order of $10^{-4}$ times less efficient than each channel of the PSD, but only one channel is taken at a time. Energy and angular resolution is defined by an aperture that is placed between the exit of the SDA and the entrance of the spin polarimeter - all electrons which fall in an energy and angular range defined by this aperture are counted as a single channel. This means that spin-ARPES data is then forced to be taken point- 

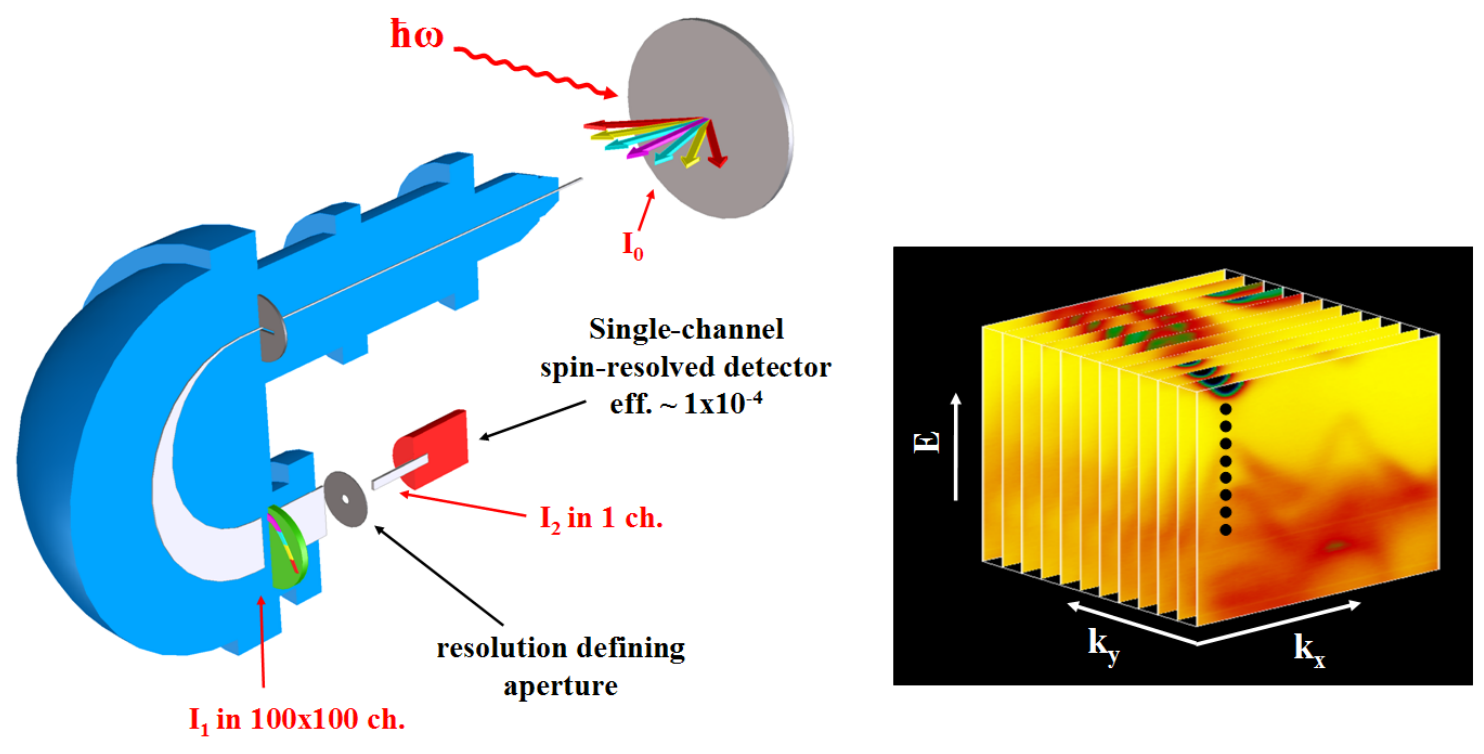

Figure 3.4. Principles of current state-of-the-art approach to spin-ARPES. The familiar and trustworthy SDA is used for energy/angle analysis. A standard multichannel PSD can take data at a count rate of $\mathrm{I}_{1}$ (at near unit efficiency) in $\sim 100 \mathrm{x} 100$ distinct channels, taking the data shown to the right in 2D slices. Spin detection is done with a single channel detector in place of the PSD, with resolutions defined by the size of an aperture at the exit of the SDA. Electrons enter the spin polarimeter at a rate of $\mathrm{I}_{2}$ as a single channel, and are detected with an effective efficiency of $1 \times 10^{-4}$. Data on the right must be taken as 0D points (black dots) one-at-a-time. 
by-point (black dots in Figure 3.4) in the multidimensional $k$ vs. $E$ phase space instead of plane-by-plane.

For illustrative purposes, let's say that we want to perform a spin-ARPES experiment with such an instrument, and we want to take as many data points with as high an energy and angular resolutions and same quality statistics as the spin-integrated version. The spinintegrated version with the PSD can take $\sim 10,000$ channels at a rate of $\mathrm{I}_{1}$ in each channel, with near $100 \%$ efficiency, for a total data accumulation rate of $10,000 I_{1}$. To achieve the same resolutions in the spin-resolved experiment, we insert an aperture that is the same size as one channel of the PSD, and install the spin polarimeter behind it. Electrons then enter the detector at a rate of $I_{2}=I_{1}$ and are detected with an effective efficiency of $10^{-4}$ for a total data rate of $10^{-4} I_{1}$. Thus, to accumulate the same data (12D slice), the spin resolved experiment will take $\sim 10^{8}$ times longer! The enormity of this difference is a large part of what has held the field of spin-ARPES back, despite the intrinsic benefits of spin-dependent measurements.

Of course the spin-ARPES experiments that have been performed did not take so long to complete. To complete a spin-resolved measurement in a reasonable amount of time (normally sample surface lifetime requires experiments to be performed in $\lesssim 1$ day), other constraints must be relaxed. Decreasing the resolutions used increases count rate as well as reduces the number of data points needed within the given data range. For instance, diminishing the angular resolution by a factor 10 can be traded for a factor 10 increase in the count rate entering the polarimeter $\left(I_{2}=10 I_{1}\right)$ by increasing the size of the aperture along the angular direction. Additionally, 10 fewer EDC's must be taken to cover the same range of angles, so the total gain is a factor 100 in regards to experiment time. Diminishing the angular resolution by a total factor of 100 and taking only a single EDC (as in the example experiments discussed in the previous section), and similarly diminishing the energy resolution by a factor 10 and taking 10 times fewer energy data points, thus gives a total gain of $10^{6}$, returning the total experiment time to near-practical levels. Further reduction in total experiment time can be made along the $k_{y}$ direction in Figure 3.4: $I_{2}$ is 


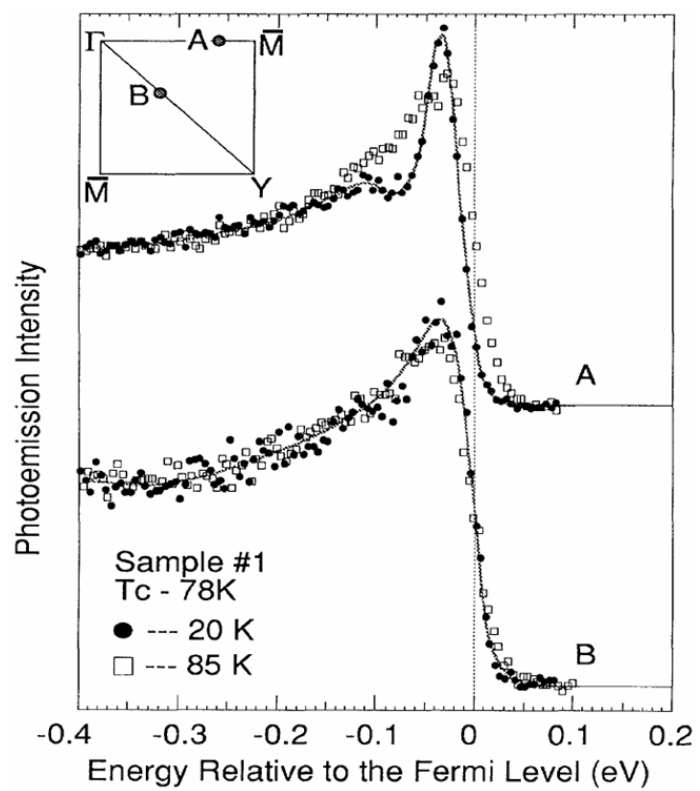

Figure 3.5. Angle-resolved EDC's at two locations in momentum space. Filled circles mark data taken below $\mathrm{T}_{C}$, while open squares mark data taken above $\mathrm{T}_{C}$. While the spectra at momentum point 'B' look very similar at the two temperatures, the spectra at 'A' show the clear opening of the superconducting gap. These measurements are a direct measurement of the anisotropy of the HTC gap. This discovery required both momentum dependence and high energy resolution to observe the changes.

further improved at the cost of decreasing $k_{y}$ resolution by increasing the vertical size of the SDA entrance slit, and fewer data steps are taken along $k_{y}$.

So we see that with current instrumental techniques, spin resolution is extremely expensive in terms of the amount of information acquired along the momentum and energy axes. Even with state-of-the-art systems, the addition of spin resolution forces the loss of the energy and momentum resolutions required to probe the interactions of central interest in current solid state physics. As a striking example, Figure 3.5 presents ARPES data from a HTC superconductor taken a decade and a half ago. ${ }^{65}$ By current ARPES standards, the resolutions were somewhat modest, but the $\sim 3^{\circ}$ and $30 \mathrm{meV}$ resolutions achieved were enough to discover the fine momentum dependence of the superconducting gap, offering strong evidence of the d-wave symmetry of HTC superconductivity. This profound discovery helped launch the enormous growth of both HTC ARPES research and the field of ARPES itself, ${ }^{30}$ and these are the energy and momentum scales that spin-ARPES must 
strive for in order to achieve similar impact. Who knows what may be discovered with additional spin resolution at similar energy and momentum scales? In order to find out, new techniques must be introduced to make definitive steps forward.

\subsection{Novel approach}

In order to make significant advancements in the field of spin-ARPES, a radically new spectrometer has been designed and fabricated from the ground up. The resulting "spinTOF" spectrometer is capable of well over 100 times higher efficiency over the current top-of-the-line systems. This is achieved through a drastic departure from the standard approaches and the introduction of entirely different working principles in two major ways.

First, energy analysis is not performed with the ubiquitous SDA. Although the SDA has been the clear choice for ARPES in the past decades due to the natural integration of $2 \mathrm{D}$ multichannel PSD's, the SDA is no longer the natural choice when forced to work with spin polarimeters that are inherently single-channel. An important concept realized in our new spectrometer, is that while current spin-polarimeter technology is necessarily single-channel along spatial dimensions, they can be made to work as multichannel detectors along the temporal dimension. The new spectrometer performs energy analysis with the time-of-flight (TOF) technique, where multi-chromatic photoelectrons disperse in time, rather than space as in deflective analyzers like the SDA. Energy analysis is performed by measuring the time the electrons take to travel from the instant of emission at the sample surface across some set distance to the ultimate electron detector. The higher the kinetic energy an electron leaves the sample with, the faster it will arrive at the detector. The key concept is that all the electrons arrive at the same spatial channel, but can be energy resolved by their differing transit times. With the TOF technique, spectrometers with single-spatial-channel detectors such as spin-polarimeters are able to acquire data along the energy dimension in parallel, becoming 1D detectors. Returning to Figure 3.4, instead of taking data in $\mathbf{k}-E$ space as single dots, the spin-TOF spectrometer can take data as vertical lines, and the number of effective channels along the time/energy dimension represents a gain in efficiency 
of well over two orders of magnitude. By moving to TOF, the new spectrometer brings the enormous efficiency of multichannel detection to the field of spin resolved photoemission.

TOF does, however, intrinsically require a pulsed light source with appropriate timing structure necessary for high resolution of the "start" times of the photoelectron's flight to the detector. This means that continuous wave (CW) sources such as helium lamps (section 2.7.4) are unacceptable. In other cases, such as synchrotron light sources like the ALS, use is restricted to operating modes which result in an overall reduction in flux of about a factor 10. Even in this case, there is still a substantial gain of more than a factor 10 in efficiency by utilizing TOF. In other cases, such as in the growing field of laserbased $\mathrm{ARPES}^{52}$ and related pump-probe time-resolved ARPES, ${ }^{79,80,81,82}$ the excitation source already has the required timing structure, and the full gain in efficiency with a TOF analyzer is realized. More details of the technique and a complete description of the custom-developed TOF analyzer is covered in the next chapter.

A second large departure from the normal spin-ARPES approach is taken with the actual spin polarimeter. The most popular type of polarimeter utilizes an asymmetry in the reflection angles from $\mathrm{Au}$ foils of photoelectrons accelerated to relativistic energies (20-100 keV). This type of polarimeter is typically called a "Mott" or also a "mini-Mott" polarimeter. Although quite common and reliable, they suffer large inefficiencies as the vast majority of the high energy electrons are not scattered by the Au foil towards the detectors or even scattered at all. The polarimeter of the spin-TOF spectrometer takes a different tack by utilizing asymmetry in the total reflectivity of low energy $(\leqslant 10 \mathrm{eV})$ photoelectrons from ferromagnetic surfaces. The low kinetic energy ensures significantly higher reflected count rates of about a factor 100 . The low kinetic energies are also very well matched to the low kinetic energies used in the TOF analyzer and produced by the UV photon energies usually used for ARPES experiments. The polarimeter design, construction, and operation is simplified by not requiring the high-voltages present in Mott-type polarimeters. Details of electron spin polarimetry and the development of the new polarimeter are covered in Chapter 5.

The combined features of the two advances in the spin-TOF spectrometer suggest total 
gains in efficiency of at least 100 over current state-of-the-art spin-ARPES methods. In experiments which already use pulsed light sources, the gain can be well over a factor 1000 . With these large gains, the spin-TOF spectrometer aims to boost the field of spin-ARPES to achieving the energy and momentum resolutions required to study the current major issues in solid state physics. The full design and initial operating experiences are reviewed in Chapter 6. 


\section{Chapter 4}

\section{Time of Flight Spectroscopy}

The advantages of TOF detection of photoemitted electrons (as well as ions) lie in the collection of the entire kinetic-energy spectrum at one time, decreasing the data collection time by as much as a factor of $10^{3}$

- V. Rehn ${ }^{109}$

Time-of-flight (TOF) is a highly sensitive, well established, and widely used spectroscopic technique based on measuring particle velocity distributions. Particles of differing average velocities are distinguished by transit, or 'flight', times through a given distance. TOF is very popular for ion mass spectroscopy and neutron scattering spectroscopies in addition to various electron spectroscopies. TOF became an attractive choice for photoemission spectroscopy at early synchrotron radiation sources to exploit the natural time structure of the synchrotrons. ${ }^{110,109,111}$ As dedicated synchrotron sources were developed with quasi-CW high repetition rates for maximum intensities for photon-hungry science, opportunities for TOF experiments decreased. The simultaneous development and success of electrostatic deflection analyzers eventually led to a period of domination by the SDA for photoemission spectroscopy (section 2.7.1). The TOF technique, however, remains very beneficial in several situations, and a number of TOF photoemission spectrometers have been developed to take advantage of certain running modes of synchrotron sources. The growing emphasis on ultrafast dynamics and time-resolved science, which require pulsed light sources with timing structures ideal for TOF methods, make TOF an especially ef- 
fective technique and has lead to increasing popularity. Spin-ARPES is a field which can particularly benefit from TOF energy analysis, and in the advanced field of time-resolved ARPES it gains enormous advantage over other techniques. Its use in the present spin-TOF analyzer can increase efficiency of spin-ARPES experiments by a factor of 10 to almost 1000 over conventional methods.

Although TOF is, and has been, well utilized in a number of photoemission spectroscopies, to the best of the author's knowledge, the spin-TOF is a unique implementation of TOF with a high precision and flexible multi-use electrostatic lens system capable of simultaneous high collection efficiency and adjustable drift velocity for high energy resolution ARPES. As we will see, this allows the high resolution to be obtained through a wide range of photon energies. Two run-time selectable flight paths allows for the choice of analysis with two detectors for differing techniques. A standard high-speed spin-integrating electron detector after a straight path can be used for fast, full spectrum ARPES to be taken, and an efficient spin-polarimeter with high-speed detection after a $90^{\circ}$ bandpass filter can be used for higher resolution, spin-resolved ARPES.

After a brief description of its working principles, the central advantages of the TOF technique are reviewed, and situations in which they are well utilized are described. A detailed account of the design, construction, and commissioning of the present TOF system is given. Key aspects of the hardware, electron detection, and timing electronics are included. The unique strengths of this particular TOF analyzer are highlighted throughout.

\subsection{TOF basics}

The TOF technique is relatively straightforward in principle, and a basic schematic of the photoemission experiment is shown in Figure 4.1. In addition to the sample and a pulsed excitation source, all that is required is a drift space followed by a high-speed electron detector. Energy resolution is provided by precise measurement of the time between an excitation pulse $\left(t_{\text {start }}\right)$ and ultimate electron detection $\left(t_{\text {stop }}\right)$. Angular resolution is provided by the system's angular acceptance, defined by an explicit aperture along the 

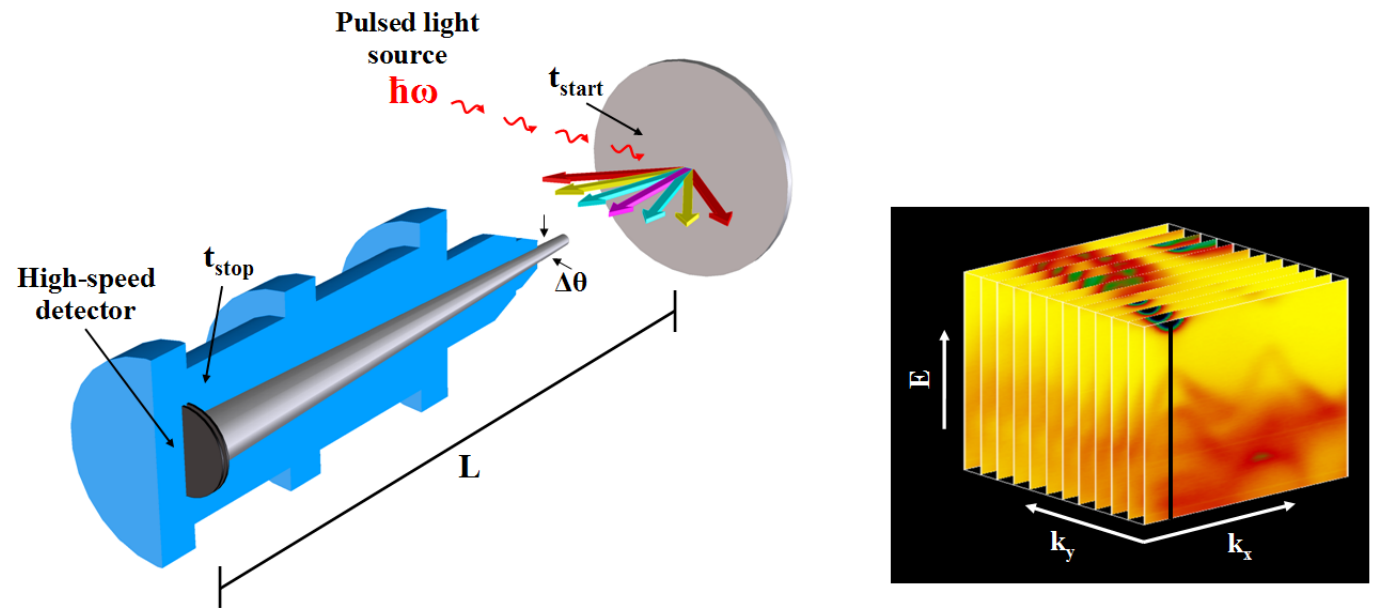

Figure 4.1. Schematic of the TOF technique, including the pulsed source, the high-speed electron detector, and the flight paths of length $\sim L$ from sample to detector. The measured time-of-flight can be converted into electron velocity and hence kinetic energy. TOF can acquire full energy spectra simultaneously as a $1 \mathrm{D}$ multichannel detector. Data in $\mathbf{k}-E$ space is taken as vertical lines on the right.

flight path or simply by the size of the detector. The kinematic relationship between electron kinetic energy and flight time is given by

$$
E_{K}=\frac{1}{2} m_{e} v^{2}=\frac{1}{2} m_{e} \frac{L^{2}}{t^{2}}
$$

where $t$ is the flight time given by $t_{\text {stop }}-t_{\text {start }}$ and $L$ is the length of the electron flight path. The distribution of photoelectron kinetic energies is measured as a distribution of flight times. Incidentally, in TOF ion mass spectroscopy, ions are accelerated by a fixed voltage $(V)$ to kinetic energy, $E_{K}=q V$, and their resulting flight times are directly related to their charge to mass ratio, $q / M$, through equation 4.1 .

TOF performance can be expressed by differentiating equation 4.1 to give

$$
\begin{aligned}
\partial E_{K} & =2 \frac{1}{2} m_{e} \frac{L^{2}}{t^{2}} \frac{\partial L}{L}-2 \frac{1}{2} m_{e} \frac{L^{2}}{t^{2}} \frac{\partial t}{t} \\
& =2 E_{K} \frac{\partial L}{L}-2 E_{K} \frac{\partial t}{t} .
\end{aligned}
$$

Assuming that the uncertainty in the measured flight times $(\partial t)$ and flight paths $(\partial L)$ are statistically independent, we see that the resolving power of a TOF photoemission 
experiment can be written as

$$
\frac{\Delta E}{E}=\sqrt{\left(2 \frac{\Delta L}{L}\right)^{2}+\left(2 \frac{\Delta t}{t}\right)^{2}}
$$

where $\Delta t$ represents the total timing resolution of the experiment, $\Delta L$ represents the path length differences of the counted electrons, and we have dropped the $K$ subscripts from the kinetic energy. Thus we see that the resolving power of the TOF experiment is limited by two main experimental parameters, timing resolution and path length.

Let us first focus on the second term on the right of equation 4.3. The total timing uncertainty of the experiment can involve a number of sources, including the light source pulse width $\left(\Delta t_{l s}\right)$, the timing resolution of the high-speed detector $\left(\Delta t_{d}\right)$, and any other timing jitter introduced by data acquisition electronics used to process the detector signal $\left(\Delta t_{d a q}\right)$. Again, assuming each of these contributions to timing uncertainty are statistically independent, we can write

$$
(\Delta t)^{2}=\left(\Delta t_{l s}\right)^{2}+\left(\Delta t_{d}\right)^{2}+\left(\Delta t_{d a q}\right)^{2}
$$

Typically, the detector speed will be the limiting factor in the total timing resolution. To ensure optimal total timing resolution, it is thus best to use a light source with pulse widths at least equal to or less than the detector speed $\left(\Delta t_{l s} \leq \Delta t_{d}\right)$. Using pulse widths much less than the detector resolution does not result in significant gain (and of course vice versa). As we will see later, in practice it is quite feasible to achieve detector resolutions of $\sim 100 \mathrm{ps}$, which conveniently is nicely matched to typical pulse widths at synchrotron lights sources.

Although elegant and concise, the $2 \Delta t / t$ expression of the timing contribution to the resolving power as written in equation 4.3 doesn't well expose the power of the TOF technique. The flight time, $t$, of a photoelectron is related to its kinetic energy as well as the path length. Ignoring the first term on the right (path length difference contribution) for now, we can rewrite equation 4.3 using equation 4.1 as

$$
\frac{\Delta E}{E}=2 \sqrt{\frac{2}{m_{e}}} \frac{1}{L} \sqrt{E} \Delta t
$$

This is an important result, as it shows that the TOF resolving power is dependent on the kinetic energy of the photoelectron being analyzed - the resolving power improves for lower 

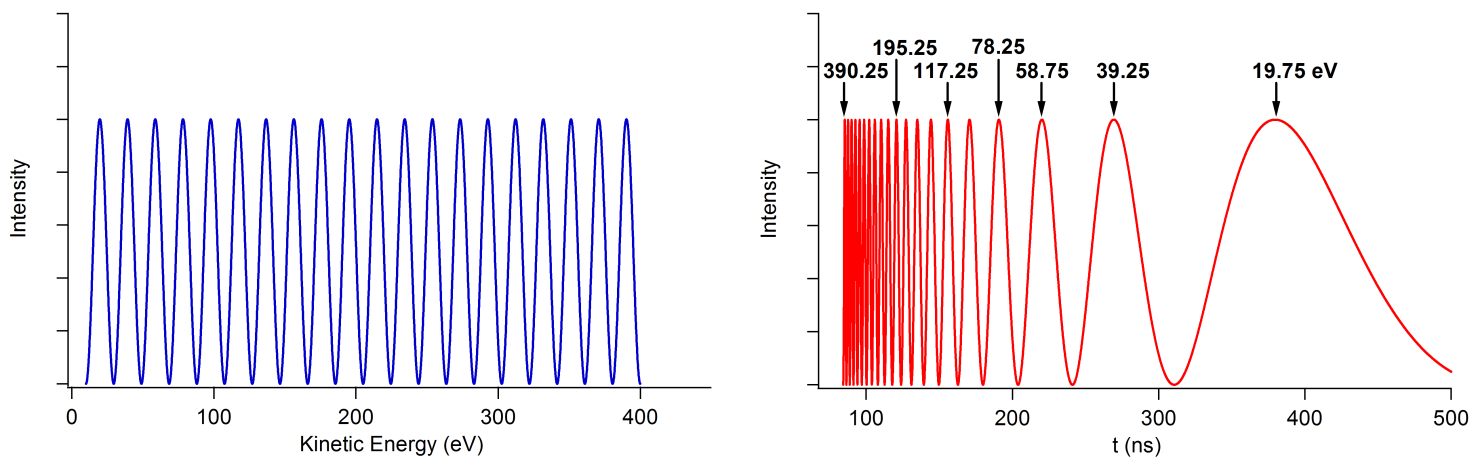

Figure 4.2. Example spectrum as a function of kinetic energy on the left and as a function of flight time on the right (with a path length of 1 meter). The spectrum is a simple $\cos ^{2}$ function in energy with fixed peak widths and separations. Several peaks in time are labeled by their corresponding energies.

kinetic energy. This can be conveniently written as an expression for energy resolution as

$$
\Delta E=1.185 \times 10^{-3} \frac{1}{L}\left(E^{3 / 2}\right) \Delta t
$$

where $E$ is in units of $\mathrm{eV}, L$ is in meters, and $\Delta t$ is in ns. The dependence of resolving power on energy is due to the fact that energy is not a linear scale in time (equation 4.1).

These concepts are illustrated in Figure 4.2. An example spectrum of photoelectrons of intensity $I(E)=\sin ^{2}(20 \pi[E-10])$ is shown as a function of kinetic energy on the left, and the same spectrum shown as a function of flight time, $t$, on the right. The spectral peaks have equal widths and separation in energy, but this is clearly not true in time, as the spectrum 'stretches out' in time as energy decreases. This has a clear effect on resolution. As an example, a system with total timing resolution of $\Delta t=10$ ns could certainly resolve the peak at $19.75 \mathrm{eV}$, but could not resolve any of the peaks above $100 \mathrm{eV}$. If resolution of these peaks at higher kinetic energy is desired, the photoelectrons can be retarded by a set potential, either by floating the sample at some positive voltage with respect to a grounded drift tube enclosing the drift space, or by setting the voltage of the drift tube and detector at some negative voltage with respect to the grounded sample. By setting the drift tube and detector to $V=-50 \mathrm{~V}$, for instance, the entire spectrum is effectively lowered in kinetic energy during the TOF analysis. Photoelectrons initially at $100 \mathrm{eV}$, are then TOF analyzed at $\sim 50 \mathrm{eV}$ average kinetic energy, thus much improving the energy resolution of 
this part of the spectrum accordingly. We see that due to equations 4.5 and 4.6 , it is often advantageous to work with low kinetic energy photoelectrons, either by using low photon energy for excitation or by retarding the photoelectrons electrostatically, for optimal energy resolution.

An important caveat relates to the repetition period of the light source. If the width of the collected time spectrum is greater that the period between excitation pulses, complications due to spectrum overlap will occur. In other words, the slowest electrons resulting from one light pulse will arrive at the detector after the fastest electrons from the next pulse, which may result in severely distorted spectra. From the arguments above, retarding a spectra will 'stretch' it out in time, increasing the effective energy resolution. However, this cannot be done without limit, as it can be stretched to widths greater than the available time window between light pulses and cause overlapping. We will return to this issue later when discussing specifics of our TOF design in section 4.4.5.

Now let us return to equation 4.3 to focus on the first term on the right hand side. This term is due to the fact that perfectly monochromatic photoelectrons will arrive at the detector at different times if the total length of their flight paths are different. Path length differences can have several contributions. One is due to finite detector size. In Figure $4.1 \mathrm{a}$ cone of electrons is eventually detected; paths along the outside of the cone are slightly longer than those along the center, introducing a path length difference. This can be minimized by decreasing the size of the detector or otherwise minimizing the acceptance angle $(\theta$ in the figure). This will also improve the effective angular resolution of the experiment, but will come at the price of decreased count rate. Due to equation 4.3, total experimental energy resolution is not greatly improved by attempting to decrease the magnitude of the 'path length' term much below the 'timing resolution' term, and so an optimal acceptance angle for good resolution and count rate can be determined for a set timing resolution. Of course, desired angular resolution may require further restriction of the acceptance angle.

Photon beam spot size on the sample also contributes to the total $\Delta L$, and its contribution greatly depends on the experimental geometry. For instance, at normal emission, the finite size of the photon beam spot does not cause much path length variation to the 
same spot of the detector if the total path length is long compared to the spot size. If $L \gg$ spot size, to a good approximation the contribution of the spot size to $\Delta L$ can be given by the component of the spot size along the central path. Thus the beam spot can become significant as the sample surface is rotated away from the normal emission geometry. As the effective illuminated spot size on the sample can also change as the sample is rotated, a careful analysis of the geometry involved should be done be sure of a small $\Delta L$.

Improved energy resolving power, $\Delta E / E$, can be improved through both terms in equation 4.3 by increasing the length, $L$. This effectively determines the physical size of the analyzer, however, and may not be practical to have an instrument above some size limit. A length of about 1 meter is typically reasonable, which limits path variation contribution to $\Delta E / E$ to below 0.001 with a total acceptance angle of $\theta=4^{\circ}$ as long as the beam spot size is also kept below 500 micron (usually not a difficult spot size to achieve).

\subsection{TOF advantages}

\subsubsection{Efficiency}

In many situations, the primary advantage of a TOF energy analyzer over deflective analyzers such as the SDA (section 2.7.1) is total efficiency. This is most obvious in cases when the deflective analyzer is utilized with a single channel detector: a single channel deflective analyzer must take 0D data point-by-point as illustrated by the black dots in Figure 3.4, whereas a TOF analyzer, even with a single spatial-channel detector, can take 1D simultaneous data line-by-line as illustrated by the black lines in Figure 4.1. This is an enormous gain in efficiency, which can be loosely quantified as the number of effective timing channels in the entire spectrum (up to 1000). Even compared to SDA's with multichannel PSD detectors, TOF offers some unique benefits. The multichannel SDA can only take simultaneous data within a certain range of energy determined by the size of the detector (and the distance between the two hemispheres) and the pass energy. The width of the energy window is typically $\sim 10 \%$ of the pass energy. Because the energy resolution is directly related to the pass energy through equation 2.38 , improved energy resolution 
requires reduced pass energy and therefore a reduced energy window. This window must then be swept through a larger range if a wider spectrum is desired, making the spectral integrity susceptible to beam or other experimental fluctuations over time. The TOF technique, however, is capable of taking the entire energy spectrum simultaneously, without the need for any 'window scanning'. This can be of great benefit for experiments which require taking spectra over large energy ranges such as those concerned with different core levels dispersed through a wide range of binding energies.

These gains may come at the expense of reduced photon flux due to the timing structure necessary for TOF analysis. Not only must the light source be pulsed, it must have a long enough period between pulses to allow the spectrum to be collected at desired resolutions. In the particular case of the ALS as the light source, this translates to a reduction of photon flux by about a factor of 10, reducing of the efficiency gains noted above. In cases such as lab-based laser sources and pump-probe based time-resolved experiments, the necessary timing structure is already required, allowing the full efficiency advantage of TOF to be gained.

\subsubsection{Sensitivity}

The TOF technique inherently relies on a delayed-coincidence measurement. Experimental noise from sources such as detector 'dark-counts' or accidental triggers are evenly spaced throughout the time range simplifying data analysis and greatly reducing the noise

contribution to each time channel. Also, this time window can be very large compared to the spectrum of interest, thus giving an even lower noise contribution to the spectrum. The detectors in TOF analyzers are true pulse-counting detectors, functioning with single-electron detection, as opposed to the intensity amplitude based measurements in most 2D SDA's. The resulting tolerable signal-to-noise ratios allow practical functioning at extremely low count rates. These characteristics as well as the total efficiency make TOF a highly sensitive technique useful for any low-count rate experiment. 


\subsubsection{Ultimate resolution}

Under certain conditions, the TOF technique can yield enhanced energy resolution compared to the SDA. From equation 2.38 we saw that the resolving power was proportional to the size of the entrance slit and inversely proportional to the mean radius of the hemispheres as

$$
\frac{\Delta E}{E} \propto \frac{S}{r_{0}}
$$

Slit size is practically limited to around 100 micron as edge effects begin disturbing too much of the passed electron beam with smaller slits. This means that improved resolving power can only come from increasing $r_{0}$, the size of the instrument. To some extent this has already been done as large $200 \mathrm{~mm}$ radius SDA's abound, but as these are already quite

large, it is not easy to envision increasing the size any further by a significant factor. In contrast, from equation 4.5 we saw that resolving power is inversely proportional to the size of the instrument, but also proportional to the square root of the kinetic, or 'pass' energy as

$$
\frac{\Delta E}{E} \propto \frac{\sqrt{E}}{L}
$$

Thus, even with a fixed instrument size, resolving power for critical features in a spectrum can be enhanced by retarding photoelectrons to lower and lower kinetic energies. With high quality electrostatic lensing, this can be performed with minimized reduction in count rate. Application of equation 4.6 using $L=1 \mathrm{~m}, \Delta t=0.1 \mathrm{~ns}$, and $E=1 \mathrm{eV}$, gives a theoretical energy resolution of almost $100 \mu \mathrm{eV}$.

\subsection{Previous uses of TOF}

TOF spectrometers first became developed to take advantage of the ideal time structure of the early synchrotron light sources such as the Stanford Positron Electron Accelerator Ring (SPEAR I) at the Stanford Linear Accelerator Center (SLAC). With a pulse width of $1.5 \mathrm{~ns}$ and a repetition period of $780 \mathrm{~ns}$, TOF provided a great option for high efficiency spectroscopy over other techniques, especially before the 2D SDA existed. Bachrach et. 
al. ${ }^{112}$ developed a straightforward TOF analyzer for solid-state ARPES composed of only a high-speed detector and a magnetically-shielded drift tube 6 inches long with a retarding entrance grid. The design parameters predicted energy resolutions on the order of $100 \mathrm{meV}$. A particular advantage of TOF in this type of setup is extreme mechanical simplicity. Due to its size and simplicity, the entire spectrometer was held inside the main vacuum chamber and was able to rotate in-situ about the sample. Early data taken with this setup was not surprisingly taken from the layered $\mathrm{TaSe}_{2},{ }^{113}$ just like the first ARPES data taken by Smith, Traum, and Di Salvo ${ }^{49,50,51}$ (section 2.3).

A similar analyzer for synchrotron-based gas phase photoemission was developed by White et. al. ${ }^{114}$ achieving almost $40 \mathrm{meV}$ energy resolution at kinetic energies around 10 eV. This design was later improved upon by Becker et. al. ${ }^{115}$ and was used because of the need to efficiently acquire data through a large energy window. Recognizing the 1D parallel detection advantage of TOF for low-count rate single-spatial-channel spin-resolved measurements, a Mott-type spin polarimeter with high speed detectors was added to their TOF design. ${ }^{116}$ This design achieved relatively good resolving powers from $0.025^{116}$ to $0.001^{117}$ with a path length of $33 \mathrm{~cm}$ and a single retardation stage. It was used for numerous experiments ${ }^{117,118}$ studying the spin-polarized Auger emission from gas phase samples using circularly polarized light (section 3.2) at European synchrotrons, before being used at the Advanced Light Source (ALS) during '2-bunch' operation for continued experiments. ${ }^{119,120,121}$ The same group at the ALS also developed a TOF experiment with four TOF systems at various angles capable of acquiring coincidence data between the analyzers. ${ }^{122}$ This instrument also capitalized on the simple mechanical nature of basic TOF analyzers by incorporating small $12.5 \mathrm{~cm}$ analyzers, all rotatable in-situ, and maximizing resolving power to $\sim 5 \%$ by utilizing a 2 -stage retarding potential.

An additional TOF system, well described by Hemmers et. al., ${ }^{123}$ has been used for gas-phase studies at the ALS. This system also utilizes four separate TOF analyzers for the enhanced efficiency of collecting multiple angles simultaneously, and each incorporates well designed cylindrical electrostatic optics for high retardation ratios while maintaining relatively high collection efficiency. 
Each of the above designs was meant for synchrotron light sources, operating in modes with relatively low repetition rates, allowing a large enough time window for the collection of the full emitted spectra. As discussed above, these operating modes involve about a factor 10 decrease in average flux compared to the higher rep-rate modes, however the larger gain in overall efficiency still makes TOF worthwhile. Pump-probe based experiments in the field of time-resolved photoemission already require a pulsed source with rep-rates which do not require further reduction for the TOF technique. For the ultra-short light pulses needed for high time resolution of the sample's dynamics, lab-based laser systems have been typically used. The ultra-low photon energies in these and other so-called 'twophoton photoemission' experiments results in very low kinetic energy photoelectrons which do not require retardation for high resolution, and allow extremely simple and short drift tube designs to be used. The high sensitivity of the TOF technique also make it an ideal choice for these extremely low count rate experiments. One particular early setup utilized a simple drift tube of $23 \mathrm{~cm}$ length to achieve energy resolutions of $100 \mathrm{meV}$ in timeresolved experiments of electron dynamics in $\mathrm{InP},{ }^{124,125} \mathrm{Si},{ }^{126}$ and other semiconducting surfaces. ${ }^{127}$ An updated system described in ref. 128 uses a $34 \mathrm{~cm}$ drift tube and a highspeed 64-channel PSD for multi-angle parallel data acquisition (effectively creating a 3D spectrometer) for energy resolutions down to $33 \mathrm{meV}$ at $7 \mathrm{eV}$ kinetic energy. Another TOF system has been developed for similar time-resolved ARPES measurements and is extremely well described by Karlsson et. al. ${ }^{129}$ with quite a bit of worthwhile details about the TOF technique in general. Yet another group has also utilized the TOF technique with $10 \mathrm{meV}$ resolution for time-resolved photoemission studying quasiparticle lifetimes in graphite. ${ }^{130}$ Straightforward TOF systems have additionally been developed for two-photon photoemission experiments studying image-potential states of metal surfaces with very high resolution of up to $5 \mathrm{meV}$. ${ }^{131,132,133}$ 


\subsection{Prototype TOF}

To get a quick start on such a big project, it was decided to begin by constructing a fairly simple prototype TOF system to allow a multitude of aspects to be tested in parallel with the designing of the larger full system. The heart of any TOF energy analysis scheme is the actual particle detector and its associated timing electronics, and the prototype system allowed these components to be developed and worked with early in the project. Before the complex spin-polarimeter was developed, we were able to get started with the TOF design by simply utilizing spin-integrating detector assemblies. An overview of the prototype system is then an ideal way to introduce these fundamental components and concepts. The prototype also involved an electrostatic lens column whose main design principles were carried over to the full system.

\subsubsection{MCP detector}

The detector technology chosen for the current TOF analyzer is based on the popular Multi-Channel Plate (MCP) electron multiplier scheme. ${ }^{134} \mathrm{~A}$ single MCP is a thin glass wafer made of a honeycomb of parallel microscopic capillaries or channels, as shown in Figure 4.3(a). The leaded-glass channels are often treated to enhance secondary electron emission. The treatment also makes the channels semiconducting; the resistivity is such that a high bias voltage can easily be held across the front and rear surfaces, but conductive enough that charge can be fairly quickly replenished from external power supplies. Thus each channel can be considered a self-contained continuous dynode. An electron incident on the front of the MCP can have enough energy to emit a large number of secondary electrons from near the front of a particular channel. These electrons will feel the strong applied potential and be accelerated along the channel, each one likely striking the sides of the channel emitting its own bunch of secondaries. This cascade continues, multiplying the number of electrons involved, until a cloud of electrons is emitted from the rear of the channel, as depicted in Figure 4.3(b). This cloud of electrons can have vastly larger total charge than the lone incident electron, and can easily be detected. 

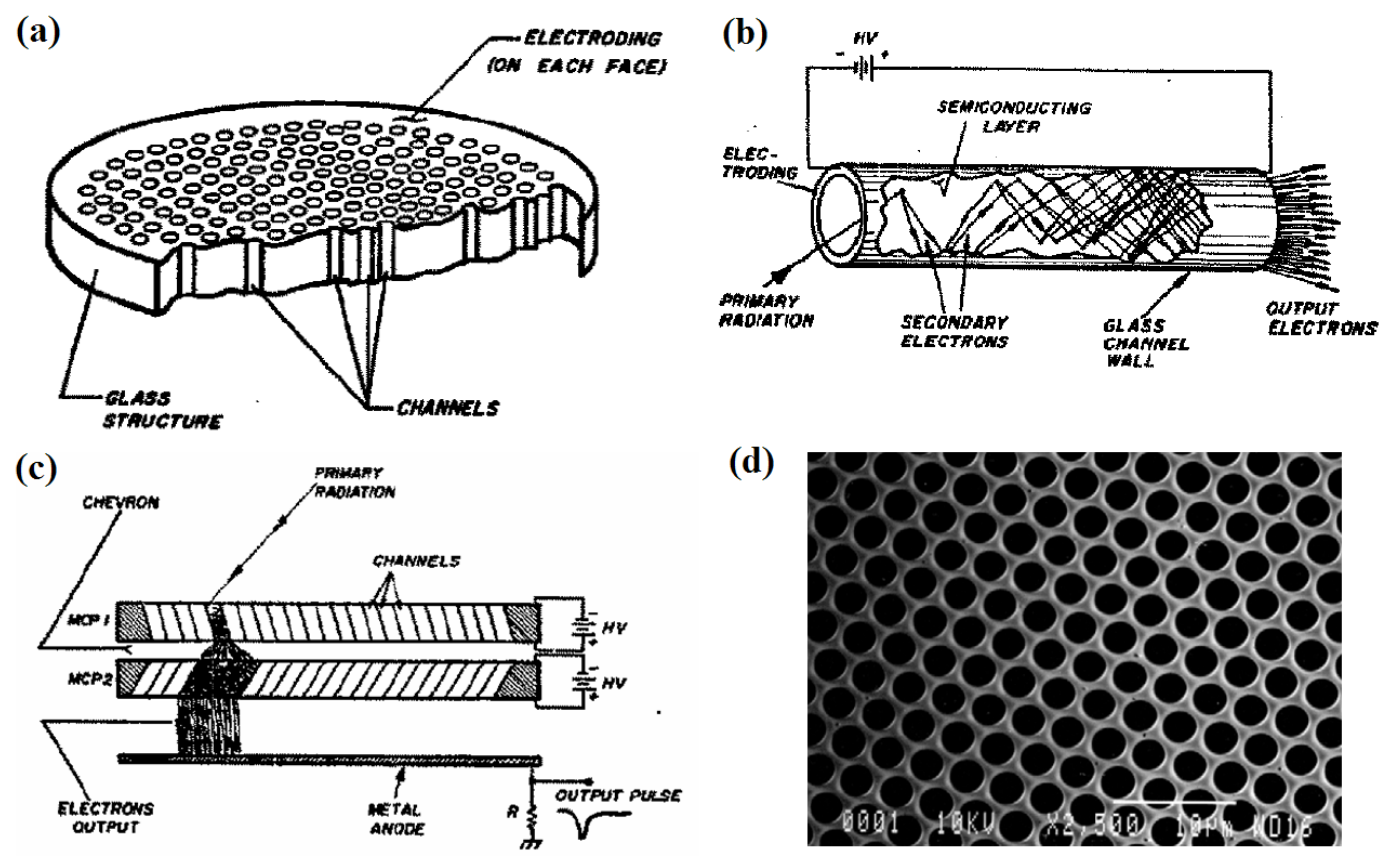

(d)

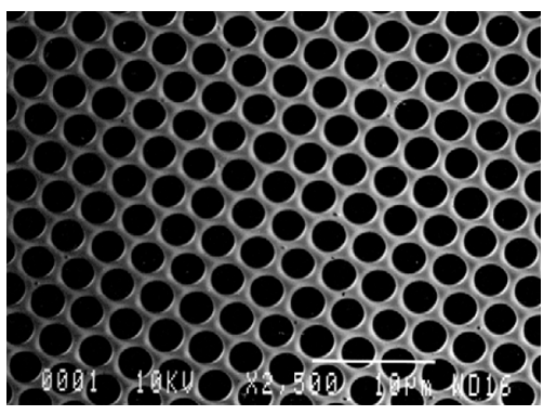

Figure 4.3. Details of multichannel plate electron amplifiers. (a) Cutaway schematic of a single MCP. (b) Cutaway schematic of an incident electron entering a single channel and the ensuing electron cascade. (c) Schematic of an MCP detector utilizing a chevron pair. The figure shows the two MCP's are electrically biased individually. Current manufacturing processes can give sets of MCP's as 'matched pairs' where the front-to-back resistivity of each is closely matched so that they can be directly touching with a single voltage placed across the entire stack. Figures (a),(b), and (c) taken from ref. 134. (d) SEM view of a $2 \mu \mathrm{m}$ pore diameter MCP produced by the Burle company. Photo taken from Burle catalog. ${ }^{135}$ 
Ideally, the channels are operated in a state of saturation where space charge effects begin to suppress the total charge output of a detection event. This allows stable operation in which detected count rates are relatively independent of slight bias voltages changes. ${ }^{134}$ Thus it is important to be able to operate the MCP at or above this saturation voltage and gain. The total gain of an MCP channel, however, can be limited on the upper end by ion feedback. Ion feedback occurs when the cascading electrons ionize residual gas molecules in the channel, or adsorbed gases along the walls, and the ions are accelerated backwards through the channel. Ions reaching the front of the channel can start a new electron avalanche, resulting in possible positive feedback scenarios that will ultimately damage the MCP.

Typically, MCPs are cut from longer 'boules' at a slight angle to the direction of the glass channels. This is referred to as the "bias" angle and helps ensure that incident electrons must strike the sides of a channel without being able to go right through. It is also common to stack two MCPs on top of each other, with the bias angle $180^{\circ}$ reversed so that there is absolutely no direct line of sight through the two. This is referred to as a "chevron stack" and helps ensure a maximum number of amplifying collisions before the cascade exits the rear, as well as helping limit possible ion feedback so that saturation can be safely obtained. Sometimes a stack of three MCPs are used, with the bias angle reversed in each to form a "z-stack". A chevron MCP detector configuration is shown in Figure 4.3(c); typically $\sim 1600 \mathrm{~V}$ is applied across the double MCP stack to reach saturation with a gain of $\sim 10^{6}$, and an additional few hundreds volts is applied between the MCP exit and the anode to direct the output electron cloud to the collection anode.

MCPs offer many appealing characteristics. First, they are fairly versatile in the type of particles they can detect. Incident electron, UV and X-ray photons, and ions can all initiate an electron avalanche in an MCP, allowing effective detection of all of the above. Second, they allow a multitude of detection output schemes. The simplest is the single metallic anode readout, as shown in Figure 4.3(c). The output electron cloud is accelerated through a potential to the anode disk resulting in a measurable discreet current pulse. Because the output electron cloud is localized to the activated channel's diameter, position sensitive 
readouts can give spatially resolved images of the incident beams. This is can be done for example by placing a phosphor screen behind the MCP and having the resulting images recorded by a camera. Because the cloud is localized to the individual channel diameter, the ultimate spatial resolutions of such setups are only limited by the channel diameters, which can now be as low as $2 \mu \mathrm{m}$. A third advantage of the MCP is that its channel characteristics such as total gain are not a function of channel diameter $(d)$ or length $(L)$ individually, but as a function of the 'aspect ratio' $L / d$, which gives maximum performance in the range of 40-60. ${ }^{134}$ This suggests that MCP's can have their overall size arbitrarily reduced as limited by mechanical fabrication techniques to create fast electron amplification. Resolution of entry time is determined by jitter in the channel transit time and the resulting pulse width which is found to be proportional to the channel length. ${ }^{134}$ Fast detectors with good timing resolution can then be made with the use of ultra short/thin channels which are quite standard in current MCPs.

Current technology can produce large MCPs with channel diameters ranging from 2 to a more standard $12 \mu \mathrm{m}$. For an aspect ratio of 40, this translates to channel lengths of less than $0.5 \mathrm{~mm}$ in the larger case. For high detector efficiency, the open area ratio (ratio of active open channel area to inter-channel wall area) must be as large as possible, and so channel density is maximized. Keeping in mind that these are thin glass structures of very thin walled tubes, they are fairly fragile. They also happen to be hygroscopic - when exposed to atmospheric moisture, they tend to soak up water and expand. Unfortunately, the stiff material usually forming a rim around the MCP for handling, installation, and overall structural stability does not expand. An MCP exposed to atmosphere for too long will warp and eventually crack. There have been so-called 'rimless' MCPs produced, but the open channels around the edges can often lead to signal ringing and are usually more fragile to the touch, and so regular rimmed MCPs are often preferred and care is taken to store them in a dry atmosphere or vacuum.

The spin-polarimeter (see Chapter 5) must ultimately rely on a single anode detector assembly, and so for testing the TOF concept in the prototype, a commercial single-anode high-speed MCP assembly was purchased from Hamamatsu, model number F4655-12. As 

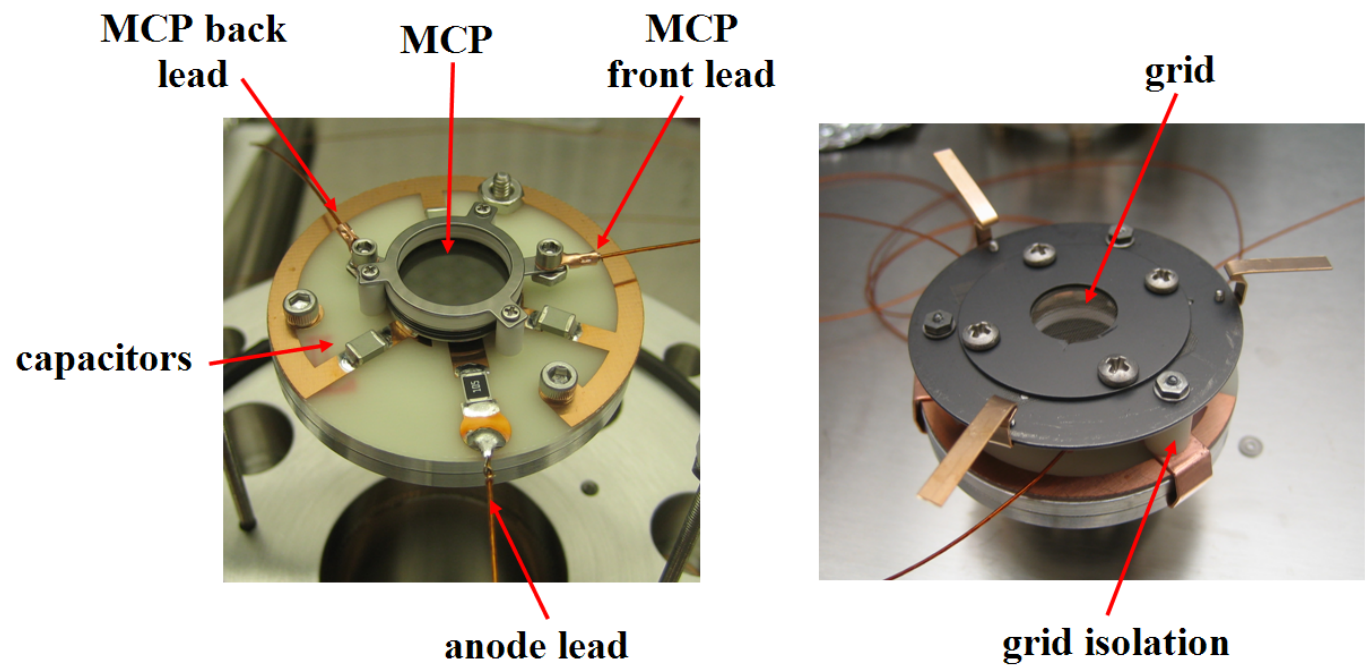

Figure 4.4. Photos of the Hamamatsu F4655-12 high speed MCP detector assembly used as a spin-integrating TOF detector. The left photo shows the base assembly with $14.5 \mathrm{~mm}$ active diameter chevron MCP stack, electrical leads for the front and back of the stack, a soldered lead for biasing the anode, and on board capacitive coupling of the anode for noise reduction. The signal out is a BNC connector on the rear of the assembly. The right photo shows the added grid assembly.

we will see, our TOF design requires the detector assembly anode to be floated to high positive voltage, but for simplicity of the external electronics, we opted to have the signal decoupled from the high voltage at the detector assembly itself. In this way, the signal is taken out of the vacuum chamber referenced to ground, and no external decoupling is required. Also, faster signals can be obtained by performing the decoupling as physically close to the source as possible. The Hamamatsu F4655-12 offers such decoupling with simple circuitry integrated into its assembly, simplifying the additional work required. The MCPs installed in the package have a $12 \mu \mathrm{m}$ pore size arranged as a chevron stack with an active diameter of $14.5 \mathrm{~mm}$. Photos of the MCP detector assembly are shown in Figure 4.4. Unfortunately, the on-board circuitry uses a plastic based substrate, visible in the photo, which should not be baked at high temperature in vacuum. Hamamatsu representatives advised to keep bakeout temperatures at the MCP to under $80^{\circ} \mathrm{C}$; all system bakeouts have been carefully monitored to keep the area of the chamber around the Hamamatsu detector at or below this temperature. Future plans may involve trying to find a different 


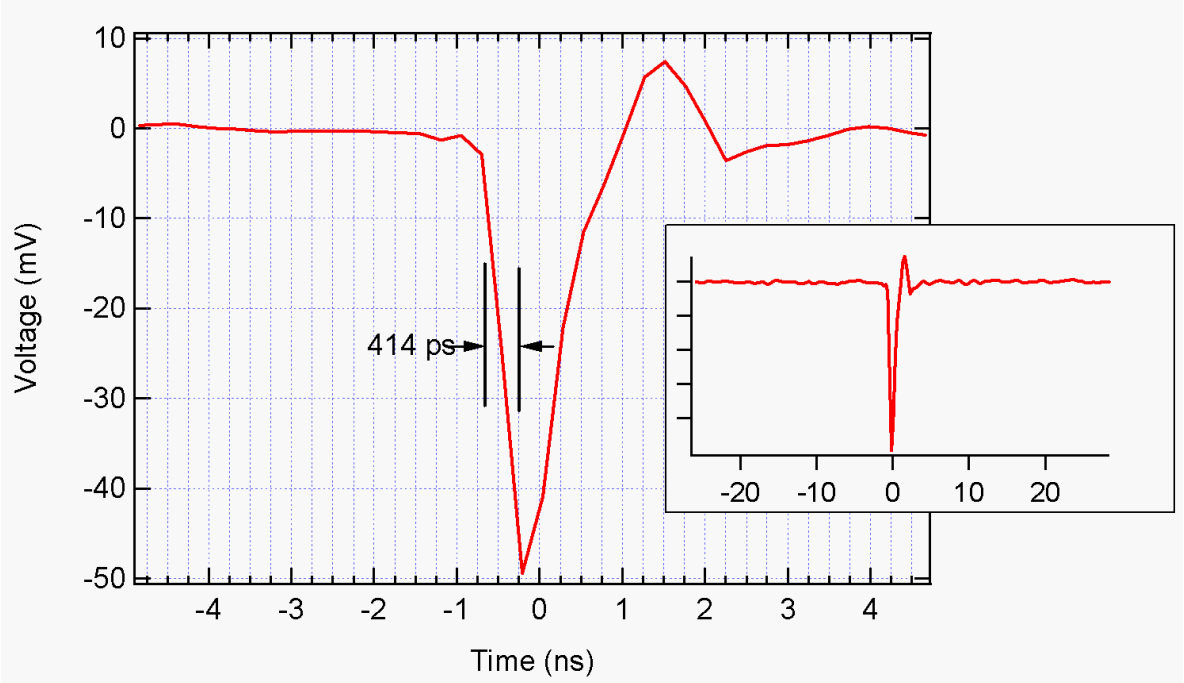

Figure 4.5. Count signal from the Hamamatsu F4655-12, measured with a $2.25 \mathrm{GHz}$ digital storage oscilloscope set at $5 \mathrm{Gs} / \mathrm{s}$. The rise time is measured as 10\%-90\% amplitude. Inset shows a wider view of the pulse, showing the very low amount of ringing after the pulse.

commercially available detector assembly which is not limited in this way, or constructing a custom one.

The Hamamatsu spec sheet claims the assembly is capable of high speed output signals with 370 ps rise time and 720 ps FWHM. Before use in the TOF system, the assembly was mounted to a simple vacuum flange for testing. Once in adequate vacuum $\left(1 \times 10^{-8}\right.$ torr is plenty), the detector could be powered on to observe the signal quality. At high enough voltages (slightly over 1600V), so-called "dark-counts" (where an electron from the front of the plate randomly field emits and causes an avalanche) can occur and can be used to monitor signal quality. Their frequency of $<1 \mathrm{~Hz}$ does not interfere with real incident electron counts during an experiment. When an electron cascade is triggered, the cloud of $\sim 10^{6}$ electrons are accelerated into the anode causing a fast current spike which is able to pass through the capacitor and onto the signal output. This signal is carried through an in-vacuum coaxial cable to a BNC vacuum feedthru, and a regular BNC coaxial cable can directly carry the signal to an oscilloscope to monitor the signal pulses, or counts. A representative signal count is shown in Figure 4.5, showing a pulse amplitude of $\sim-50 \mathrm{meV}$ 
with a rise times of $\sim 400 \mathrm{ps}$, fairly close to the specifications claimed by Hamamatsu, and are plenty fast enough for the timing necessary.

\subsubsection{Timing electronics}

The signal from the MCP detector marks the time of electron arrival at the detector; it forms the 'Stop' signal in the TOF analysis. To do this as precisely as possible, the signal must be put through some basic signal processing. First, the signal is sent through a 'preamplifier', converting the current pulse to a strong voltage pulse for further processing. It is better practice to send the signal directly from the chamber vacuum feedthru into the preamp with a short a cable, so that the pulses can be as clean and noise free as possible before being enhanced in the preamp.

A precise and reproducible arrival 'time-stamp' must then be extracted from this signal. A time pick-off circuit is used to output a digital pulse which is consistently related to the beginning of each input signal in time. Because the signal pulses from the MCP can have a wide range of amplitudes from count to count, and can vary in rise times and shape, the time-stamping should be done in a way which is as insensitive to these variables as possible. How this is handled can have a large impact on the system timing resolution. For example, the most straightforward approach would be leading-edge threshold triggering, where the pulse from the preamp triggers a digital output signal when a certain threshold voltage is reached. As illustrated in Figure 4.6(a), output triggering in this method is a function of amplitude and rise-time, varying the digital output time relative to the actual detection time. This is referred to as 'walk'. Threshold triggering also suffers from 'jitter', or time uncertainty due to noise in the signal input which can alter when the signal crosses the set threshold.

An alternate method is illustrated in Figure 4.6(b) where the triggering occurs at the same exact time for both signals, achieved by triggering at a certain fraction of the total pulse height. This can be done with an accurately named Constant Fraction Discriminator (CFD). A CFD works as illustrated in Figure 4.6(c): the input pulse (red) is duplicated, 

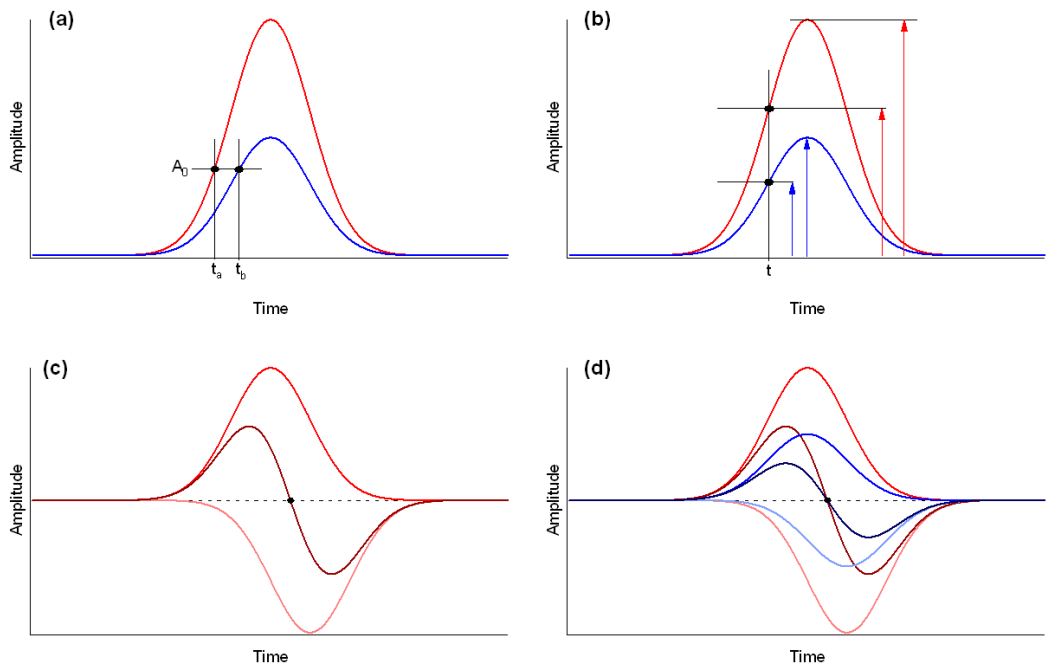

Figure 4.6. (a) Leading edge threshold triggering. The time difference between $t_{a}$ and $t_{b}$ is the walk. (b) Triggering at a constant fraction of pulse amplitude, giving zero walk for pulses of different pulse heights, but equal rise-times and widths. (c) Operation of a CFD. The input pulse (red) is duplicated, inverted, attenuated, and delayed (pink), and the sum of the two (dark red) has a well defined zero-crossing point for triggering. (d) CFD applied to the two pulses of (a) and (b). The zero-crossing points are identical, giving a triggering time independent of input pulse amplitude.

slightly attenuated, inverted, and delayed (pink), and the two are then added together (dark red). A digital output signal is triggered at the resulting zero-crossing point. This process is shown for two different input pulse amplitudes in Figure 4.6(d), illustrating that the CFD triggers at the same relative time independent of input pulse amplitude. Output walk from amplitude as well as rise-time variations can be well minimized by tuning the delay, and jitter can be minimized by tuning the attenuation. A CFD commonly features a leading edge armed discriminator requiring the input pulse to reach a tunable threshold to allow any output signal. This provides selection of a minimum acceptable pulse height and keeps the sensitive zero-crossing circuit from triggering on noise. To keep noise at a minimum, it is best to perform CFD very close to the preamp. A more complete review of time-pickoff techniques can be found in ref. 136.

The current TOF project utilizes a very convenient and effective commercial package (the Ortec 9327) which combines a fast $1-\mathrm{GHz}$ preamplifier and a high quality CFD in a single small unit. The small size and portability of the 9327 allows it to be placed right 
next to the MCP output vacuum feedthru so that the signal processing and digitization can be performed extremely close to the signal source. The digital output (a fast 'NIM' pulse with amplitude $\sim-800 \mathrm{mV}$ ) time-stamp can then be safely transmitted over much longer cables. The preamp in the 9327 provides two course gain settings for input amplitudes of either 0-30 $\mathrm{mV}$ or $0-150 \mathrm{mV}$, and both have been needed for the spin-TOF detectors. Gain can additionally be fine-tuned over a range of $2: 1$. If input pulses are too large and saturate the preamp, an overload indicator lights, and the gain can be turned down appropriately. Adjustment of the discriminator threshold can be performed by blocking all sources of real electron events and turning up the threshold to slightly beyond when all output pulses stop, ensuring that noise is not triggering outputs. The 9327 CFD keeps walk to less than 40 ps and jitter to less than 20 ps.

In timing experiments, the digital CFD output pulse is sent to a time-to-amplitude converter (TAC), which outputs a pulse whose amplitude is a linear function of the time between some input 'start' signal and the input 'stop' signal from the CFD. The TAC output signal with the precise time between 'start' and 'stop' encoded in its amplitude is then sent to a single channel analyzer (SCA) or an array of them called a multi-channel analyzer (MCA). These devices keep track of the number of incoming pulses whose amplitude is within some range defined for each channel. Again, the current TOF project uses an extremely convenient package from Ortec which combines the functionality of a high-precision TAC and MCA into a single unit, the '9308-PCI picosecond Time Analyzer'. The 9308 accepts input NIM logic pulses for both 'start' and 'stop' signals. The unit is capable of multiplestop recording with 16 -bit resolution over time windows ranging from 80 ns to $325 \mu \mathrm{s}$. The 'start' signal, corresponding to the incident photon excitation pulse, defines $\mathrm{T}=0$ and starts a timing scan; the 9308 then records an incoming 'stop' signal as an event in one of the 65,536 channels, or bins, corresponding to its time of arrival at T=t. Many series of 'start' and possible 'stop' signals can be processed sequentially, adding the number of events in each bin, forming a histogram of photoelectron flight times. This repetitive sampling can be continued until the required statistical precision is obtained.

The 'start' signal must be a fast NIM logic pulse which is precisely related in time to the 


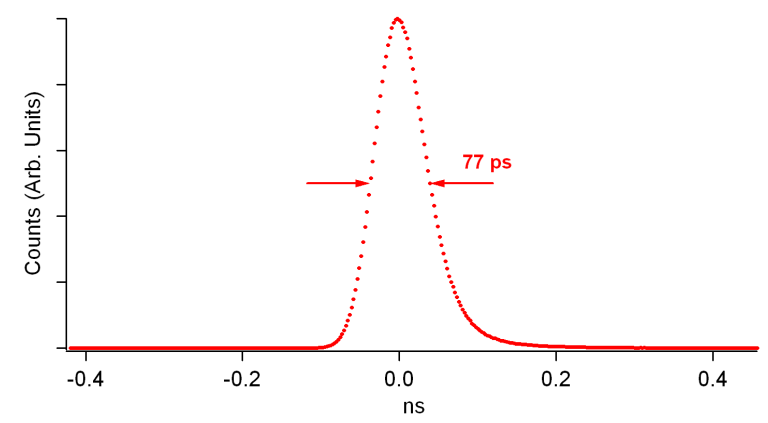

Figure 4.7. Time spectrum of the $\sim 200$ fs mode-locked laser. The width of the spectrum represents the total timing resolution of the detector and electronics.

excitation light source pulses. In the case of synchrotron light sources, timing electronics in the storage ring can provide a reliable reference. For laser-based sources, a fraction of the beam can be used to excite a fast photodiode to be used as reference for a 'start' signal. Once a scan is started, the 9308 will only record 'stop' signals and ignore any other 'start' signals until the set time window, or time span, is passed. After a slight deadtime after the end of the time span, the 9308 will ignore all 'stop' signals until another 'start' signal starts the process again. The time span can be set for as small as $80 \mathrm{~ns}$; in this case the 16-bit precision gives a bin width (digital resolution) of 1.221 ps. The time span (and hence bin width) can be increased by factors of 2 to a maximum of $325 \mu$ s. The 9308 can also be configured to delay the beginning of the time span by a selectable offset for the 'start' time. Resulting histograms are sent from the 9308 unit to a personal computer through the provided PCI card. User commands and configurations are also performed through the computer interface.

The high performance of the 9327 preamplifier and CFD and 9308 time analyzer means that the MCP detector itself, or possibly the light source pulse width, is the dominant source of timing uncertainty and is the limitation in timing resolution. The 9327 and 9308 combination perform their duties with only a few BNC cable connections, making the setup remarkably simple despite its high performance. The ease-of-use of these devices makes TOF-based experiments much easier than they once were. The layout of the TOF photoemission experiment based on these electronics components is illustrated in Figure 4.10. 
The total timing resolution of the MCP detector and signal processing electronics can be conveniently measured by simply exciting the MCP with a pulsed laser source and measuring a spectrum with the above electronics. The $6 \mathrm{eV}$ th harmonic generated photons are easily detected by the MCP, and the $\sim 200$ fs pulses effectively remove any source contribution to the measured peak width. The mode-locked laser has a fast-photodiode to provide a reliable 'start' signal. The resulting spectrum is shown in Figure 4.7. Because the light pulses are 300 times shorter in time, the measured $77 \mathrm{ps}$ pulse width is accurately representative of the timing resolution achieved by the detector and processing electronics. As noted above, this is likely mostly dominated by the MCP detector itself. Timing uncertainty due to the MCP detector has contributions from jitter in the electron avalanche transit time through the channels, as well as uncertainty in the location of the signal on the detector. The signal is only taken from the anode disk in one point - the signal pulse absorbed closer to the edge of the anode will have to travel a longer distance to the signal output than one absorbed closer to the output, introducing a timing spread of $\sim \Delta d / v$, where $\Delta d$ is the difference in possible distances from detection point of the anode to the signal pickup lead, and $v$ is the signal velocity along the anode. In a somewhat similar situation, Vredenborg et. al. ${ }^{137}$ estimated the signal velocity to be $\sim 0.7$ times the speed of light. For the present case, with the signal lead in the center of the $14 \mathrm{~mm}$ diameter detector, we then have $\Delta d / v \approx 0.007 /(0.7 c) \approx 33$ ps. Thus, this is likely a less important contribution than the MCP transit time to the total timing resolution. This contribution could be decreased by using smaller channel diameter MCPs, resulting in thinner plates, although these are then even more fragile and prone to physical damage. The current TOF system, however, is meant primarily for use with the ALS which has 70 ps width light pulses, so even with improved timing from the detector, the overall timing resolution will not be significantly enhanced.

\subsubsection{Lens column}

We saw in section 4.1 that TOF energy resolution is greatly improved towards lower electron drift energies. This is automatically achieved when using low photon energies such as with laser-based sources, where the maximum photoelectron kinetic energies are only a 


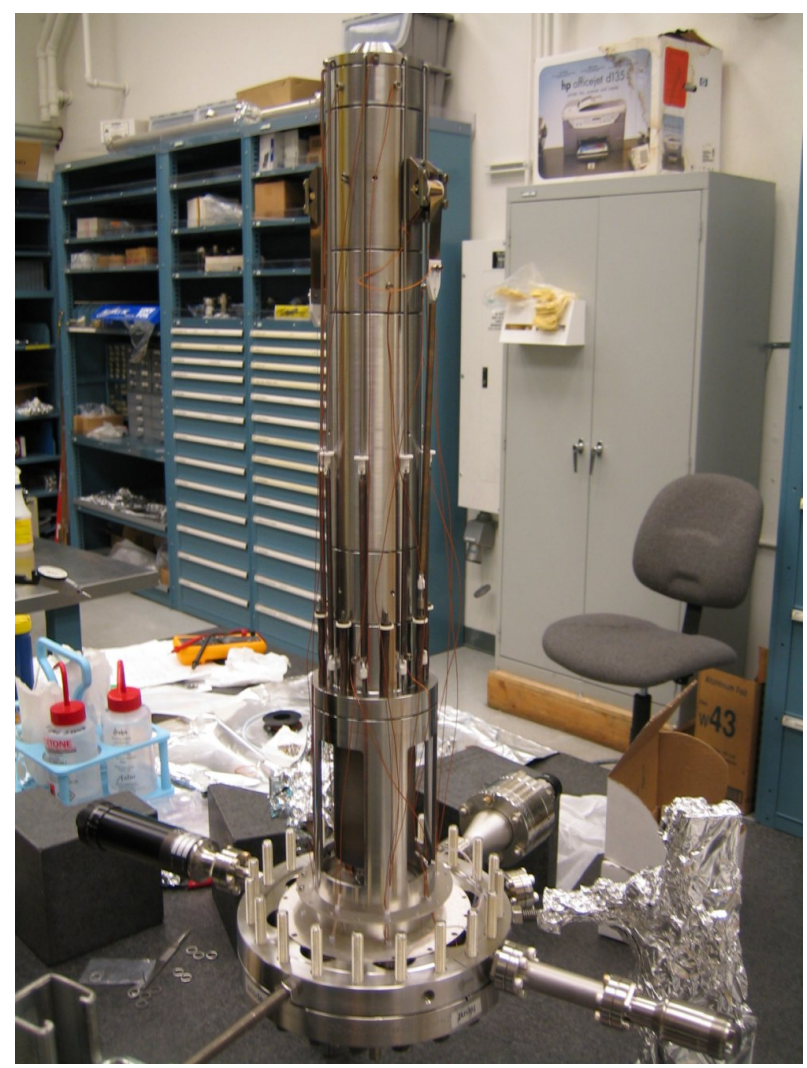

Figure 4.8. Photograph of the assembled prototype TOF lens column.

couple eV to begin with. However, using higher energy sources such as synchrotron light offer increased Brillouin zone range, a different set of matrix elements, and wide tunability, all of which can be crucial depending on the science being investigated. Higher photon energies means higher photoelectron kinetic energies, which translate to decreased TOF energy resolution. Electrostatic retardation of the photoelectron spectrum can be applied to reduce the effective electron drift energy. Simple one-step retardation schemes such as in previous TOF analyzers (see section 4.3) can greatly reduce efficiency as the accepted photoelectron beam can be defocused. The goal of the present TOF project is to provide a high-quality electrostatic lens system capable of tuning the effective drift energies for the desired energy resolution while maintaining good focus for maximum collection efficiency. To begin understanding basic lens system capabilities and experiment with the general construction design to be used, a prototype lens column was designed for use with initial testing of the Hamamatsu MCP detector assembly (section 4.4.1). 
The prototype lens column is a stack of 7 electrically isolated coaxial cylindrical electrodes. Each lens was originally isolated by six small ceramic cylinders placed between each electrode at their radii. The stacked column of electrodes and ceramics are then compressed together by eight axial spokes tensioned by springs to form a mechanically rigid single column. Many details of the prototype concept and design were kept in the final TOF lens system, and these will be detailed in section 4.5. Figure 4.8 shows a photograph of the assembled prototype lens column before its insertion into magnetic shielding and its vacuum chamber. Photoelectrons enter the lens column at the top of the photo, and the MCP detector is installed from the bottom.

\subsubsection{Beamtime, data acquisition, and time-to-energy conversion}

As mentioned briefly in section 4.1, the TOF technique is best done when the entire time spectrum from fastest photoelectron to slowest recordable photoelectron can fit within the period between light pulses. The ALS storage ring normally operates in 'multi-bunch' mode, where there are $\sim 328$ distinct electron bunches orbiting the ring 2 ns apart. Each bunch is $\sim 70$ ps wide. When each bunch passes through a given insertion device or bend magnet, a corresponding pulse of radiation is sent down the beamline. Thus, a beamline provides radiation in 70 ps pulses at $500 \mathrm{MHz}$ during multi-bunch mode. The small $2 \mathrm{~ns}$ window between pulses is not enough to perform a reasonable TOF experiment without severe overlapping. For example, a $50 \mathrm{eV}$ electron takes 238.616 ns to travel a distance of $1 \mathrm{~m}$. A $10 \mathrm{eV}$ electron takes $294.945 \mathrm{~ns}$ longer. Therefore, for a straight TOF analyzer $1 \mathrm{~m}$ long, there must be at least $295 \mathrm{~ns}$ between light pulses to take a spectrum of photoelectrons with kinetic energies ranging from 50-10 eV. Clearly, the TOF technique is not very well suited for multi-bunch operation at the ALS or other high rep-rate light sources.

The ALS also operates in '2-bunch' mode, where there are only 2 bunches orbiting the storage ring, separated by $328 \mathrm{~ns}$. This provides a $328 \mathrm{~ns}$ window in which to perform TOF

and other time-related experiments which require smaller rep rates. Reducing the number of bunches from 328 to 2 suggests a 164 times reduction in overall photon flux. However the ALS inserts more charge into each bunch during 2-bunch mode so that the reduction 


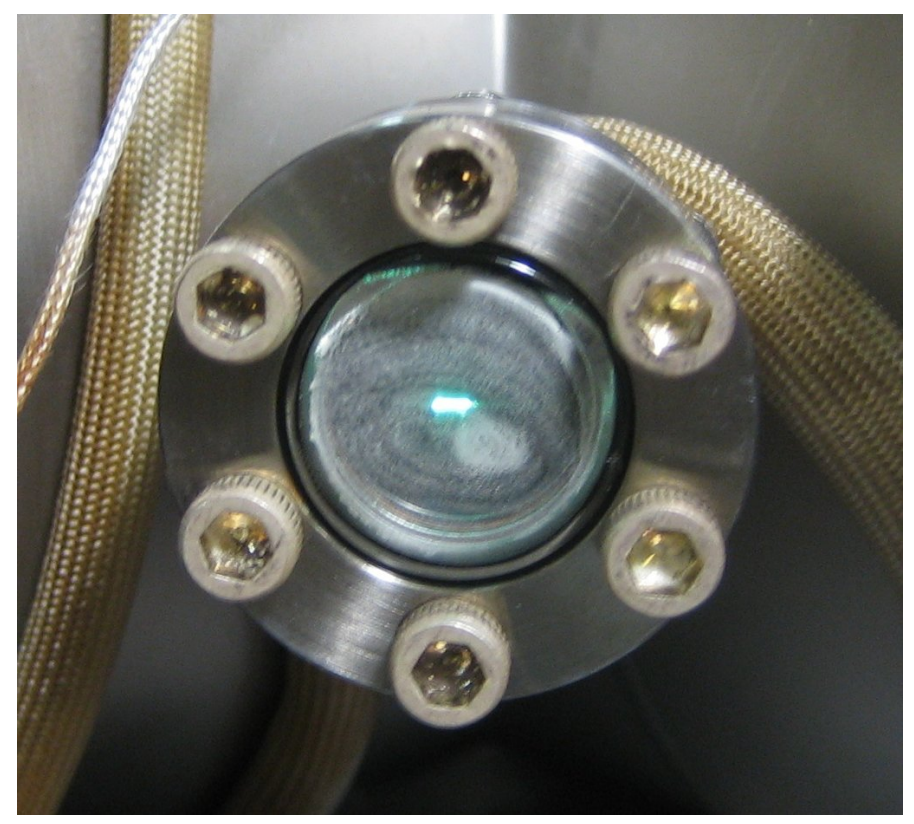

Figure 4.9. Photograph of the phosphor coated mini-viewport on the analysis chamber illuminated by the beam, illustrating the beamspot.

in total flux is only about a factor 10. Unfortunately, the larger total charge in each bunch decreases its lifetime in the storage ring, so the photon flux decreases fairly rapidly and storage ring refills must occur every 2 hours. Over the two hours, the flux decreases by about a factor 5 .

The ALS typically operates in 2-bunch mode for 2 weeks, twice a year. A number of opportunities were available to install the TOF prototype on a small UHV analysis chamber downstream of the ARPES endstation (run by Alexei Fedorov) at beamline 12.0.1. This undulator beamline features a monochromator with a 300 lines/mm grating that can produce a range of usable photons from about $30 \mathrm{eV}$ to $90 \mathrm{eV}$, with maximum flux around $60 \mathrm{eV}$. Although other gratings are available, this is the one which was used for TOF experiments. Unfortunately, the last horizontal mirror is mechanically unable to flex to the radius needed to give a sharp focus in the downstream position, leading to a very unfocused beamspot at the sample position in the horizontal direction. The TOF analysis chamber has a 'mini' sized viewport directly opposite the beam input port, coated on the inside with a thin, uneven film of phosphor which shows the beamspot at that position when the sample is removed from the beam. A photograph of the viewport illuminated by the beam 


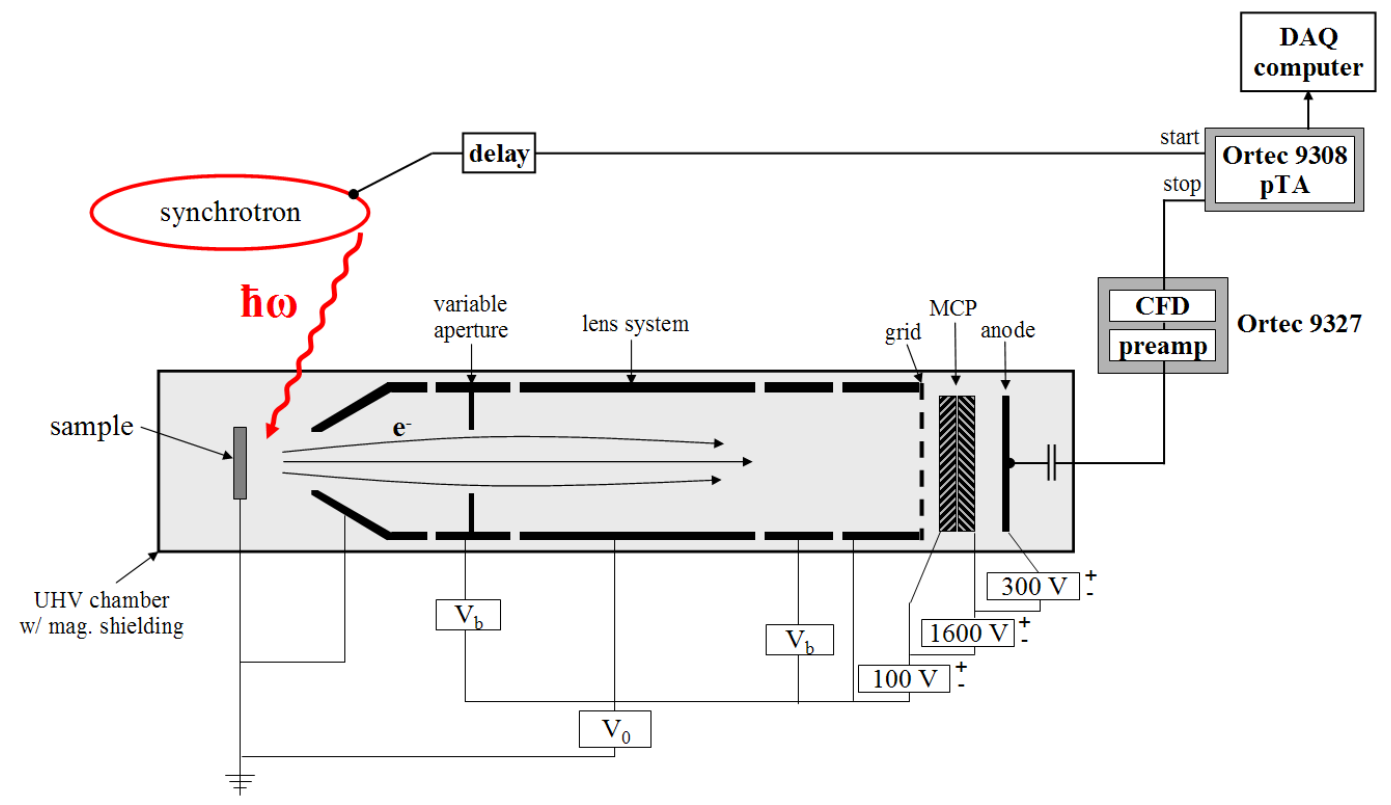

Figure 4.10. Schematic layout of TOF photoemission experiment.

is shown in Figure 4.9, showing a beamspot of just under $3 \mathrm{~mm}$ horizontally, and just under $1 \mathrm{~mm}$ vertically. The horizontal size represents the best obtained, while the vertical was usually a smaller fraction of $1 \mathrm{~mm}$, closer to a couple hundred micron.

A schematic of the experimental layout is shown in Figure 4.10, including the sample, a simplified diagram of the lens column, the MCP detector, and signal processing electronics discussed in section 4.4.1 and 4.4.2. The beamline is equipped with a 'User Timing System User Control Chassis' which provides a fast NIM digital pulse at times referenced to when electron bunches pass a certain point in the storage ring. These pulses can be delayed by selectable amounts, although the exact setting is unimportant as it is only a reference time marker, and does not represent an absolute time of the photon pulse anyway. This signal output on a beamline electronics rack is simply connected via a long BNC cable to the 'start' input of the Ortec 9308, as shown in Figure 4.10. The only detector related cabling is as follows. A short ( 6 inch) coaxial cable connects the BNC feedthru of the MCP signal output to the SMA input of the Ortec 9327 which is mounted directly to the vacuum chamber. The NIM output of the 9327 is then connected via BNC coaxial cable 
to the input of the 9308 , and a long ribbon cable assembly connects the 9308 to the data acquisition computer.

The sample in the initial experiments was simply a Au film, freshly evaporated in-situ in vacuum of less than $1 \times 10^{-10}$ torr directly onto a copper block bolted to the end of a liquid helium cryostat. This gives a clean polycrystalline Au surface with minimum difficulty which can be lowered to $\sim 20 \mathrm{~K}$.

The simplest way to take data is to leave the entire lens column grounded, producing a simple field-free drift length from the sample to the detector. With the beamline set to $h \nu=60 \mathrm{eV}$ the spectrum in Figure 4.11 can be quickly taken. A few things can be immediately observed in the spectra. First, the tallest peaks qualitatively look like the expected two peak structure of the strong $\mathrm{Au}$ valence band, and this is strengthened by what appears to be a sharp edge on the shorter time side, corresponding to the Fermi edge. Second, there is an extremely sharp single peak on the far left of the spectrum, which is due to photons scattering from the sample and triggering the MCP detector. This interpretation is supported by the following observations. The lens column can easily be set to very high negative voltages such that all photoelectrons are repelled and none can reach the detector. When this is done, all counts except for this sharp peak disappear from the spectrum, which is what is expected for a peak whose origin is photons. Also, the peak width is extremely sharp $(<100 \mathrm{ps})$ corresponding well to the width of the bunches in the storage ring producing the light pulses. Finally, if the sample is rotated to an angle such that specular reflection is directed towards the detector, this peak intensity grows quite large. The fact that the photons result in a nice sharp peak is extremely convenient, as we can use this peak to calibrate the time scale such that this peak is set to $T=0+L / c$, where the second term corrects for the finite amount of time it takes the light to travel the length of the analyzer.

Of course the real goal is to collect a spectrum in energy, not in time, and so a conversion must be performed. Because of the field-free drift space, this conversion can be done according to $E_{K}=\frac{1}{2} m_{e} L^{2} / t^{2}$ (equation 4.1). A slight complication arises due to the fact that this is not a linear conversion. As introduced in section 4.1, an energy spectrum 


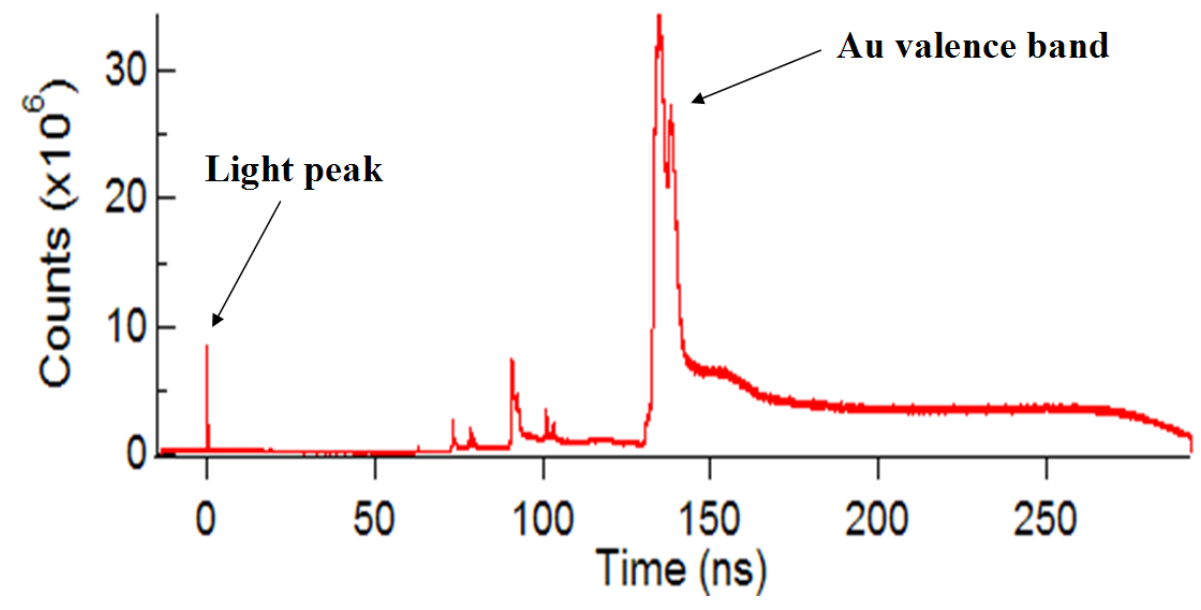

Figure 4.11. Au time spectrum taken with $h \nu=60 \mathrm{eV}$ and the lens column grounded.

stretches out in time at lower kinetic energies (Figure 4.2), effectively improving the energy resolution at lower kinetic energy. Bin widths experience the same effect: low time (high energy) bins span a larger range of kinetic energy compared to large time (low energy) bins which span a smaller range of kinetic energy. This means that count totals must be normalized to account for the energy bin density increasing towards lower energy (larger time). Figure 4.12 helps illustrate this point. The left side of the figure presents a time spectra consisting of equally spaced, equal width and height square waves. Correct conversion to an energy axis requires normalization to maintain constant 'peak' areas, giving the energy spectrum shown on the right. Normalization is achieved by multiplying by the Jacobian of the $E=\frac{1}{2} m_{e} L^{2} / t^{2}$ conversion $^{138}$ given by

$$
\Delta E=\frac{2 E}{t} \Delta t
$$

In this way, the intensity of the energy spectrum is normalized according to

$$
I\left(E_{\tau}\right)=C(\tau) \times \frac{\tau}{2 E_{\tau}} \frac{1}{\Delta \tau}
$$

where $I\left(E_{\tau}\right)$ is the correct intensity of the energy spectrum at energy $E_{\tau}$, corresponding to a flight time of $t=\tau$. $C(\tau)$ is the total number of measured counts in the time bin at $t=\tau$, and $\Delta \tau$ is the bin width. This can also be expressed as

$$
I\left(E_{\tau}\right)=\frac{C(\tau)}{E(\tau)-E(\tau+1)}
$$



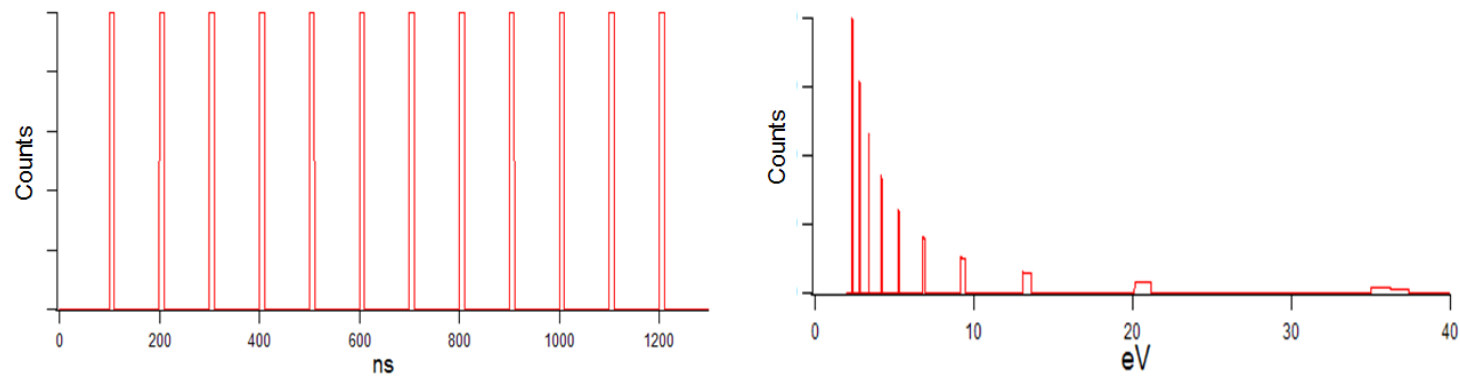

Figure 4.12. Converting the time spectra on the left to the energy spectra on the right, correcting for non-linearity of bin density.
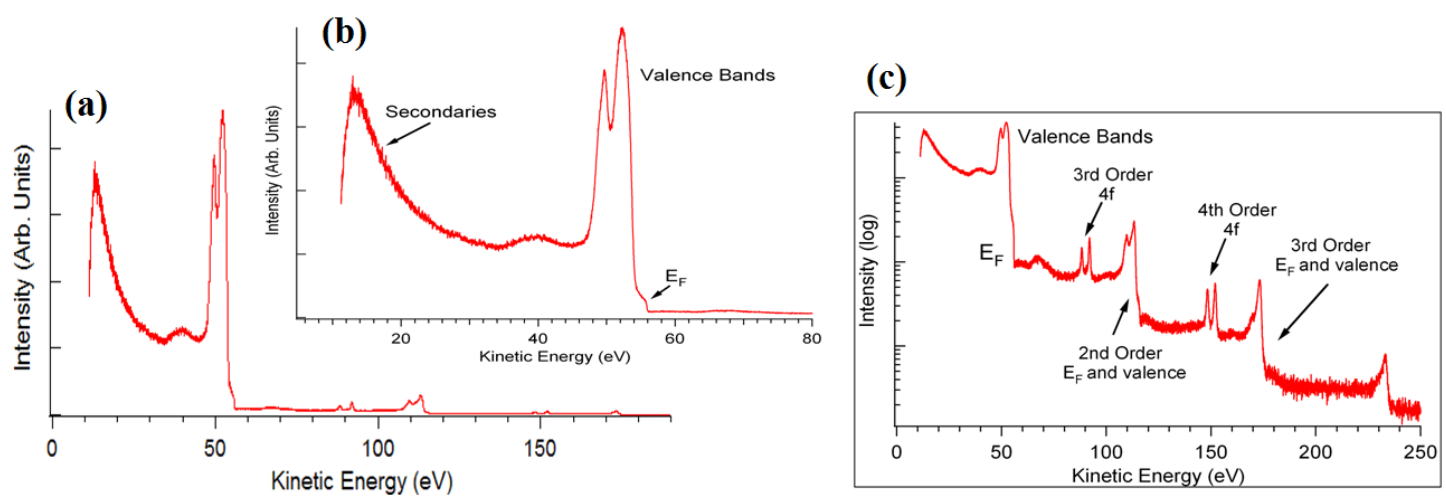

Figure 4.13. The Au energy spectrum, converted from the time spectrum in Figure 4.11. (a) Full spectrum. (b) Spectrum from 0-80 eV. (c) Log scale plot of full spectrum.

where $E(\tau+1)$ is the energy corresponding to the flight time of the bin directly adjacent to $t=\tau{ }^{123}$ The effect on widths and heights is clearly seen in Figure 4.12.

Applying this conversion process to the time spectrum in Figure 4.11 results in the energy spectrum in Figure 4.13(a). This gives much reassurance that everything is as it should be. A zoomed in view of the spectrum below $60 \mathrm{eV}$ is shown in panel (b). The valence band looks just as one would expect, with a nice sharp Fermi edge at around $55 \mathrm{eV}$ where it should be $(h \nu-\Phi)$. The bin width correction shows that the low energy spectra in fact increases quite rapidly as one expects from the increasing secondary background.

Panel (c) shows a log plot of the entire energy spectrum such that the smaller peaks at higher kinetic energy stand out more. Naively, one would not expect any counts above $\mathrm{E}_{F}$, however one must remember that the beamline monochromator can pass multiple harmonics 


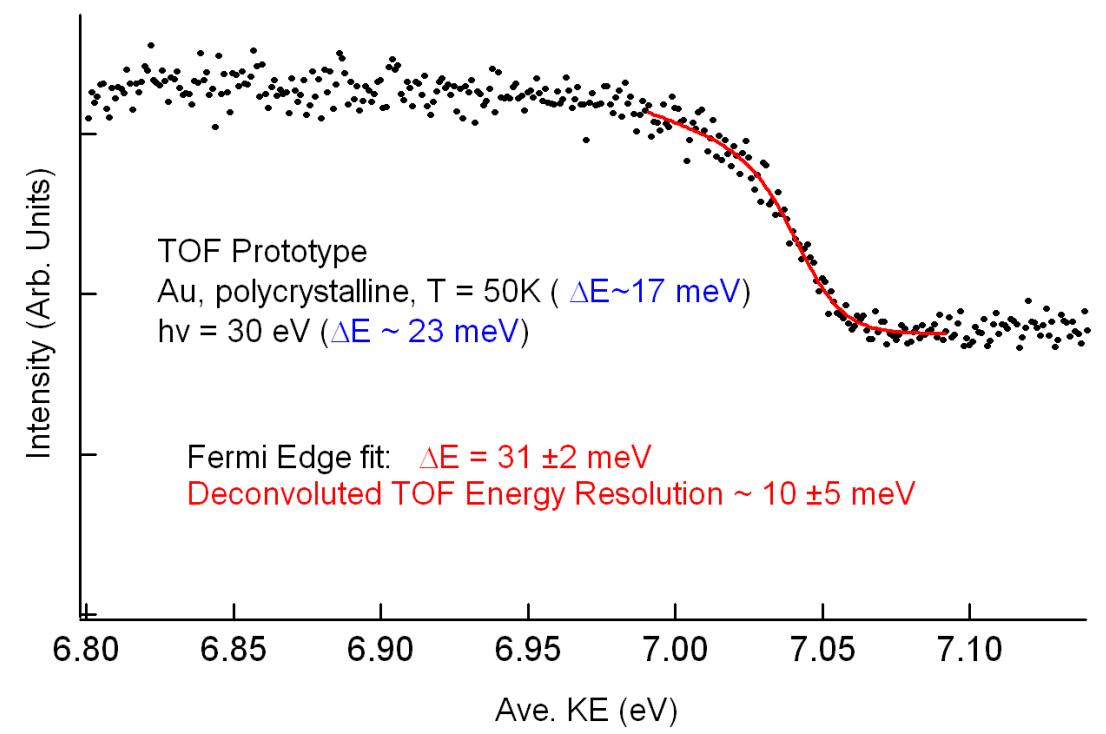

Figure 4.14. Au Fermi edge spectrum with $h \nu=30 \mathrm{eV}$, retarding $\mathrm{E}_{F}$ to about $7 \mathrm{eV}$ kinetic energy.

of the desired energy. While the majority of photons incident on the sample are $60 \mathrm{eV}$, there are also some at $120 \mathrm{eV}, 180 \mathrm{eV}$ and so on. This causes the simultaneous spectra at these higher photon energies as well, with the Fermi edge of each showing up at $55 \mathrm{eV}, 115 \mathrm{eV}, 175$ $\mathrm{eV}$, and so on. This is exactly what is seen in the spectra. In fact, this is quite convenient as precise conversion relies on knowledge of the exact flight path length, L. The value of $\mathrm{L}$ can be adjusted in the conversion process to make each Fermi edge show up exactly $h \nu$ apart. The fact that they can all be correctly adjusted with a single value of $\mathrm{L}$ confirms that the process is a good one. Additionally, these higher photon energies are enough to reach the well known $\mathrm{Au}$ if core doublets, labeled in the figure. These are well studied and their splitting is well known, giving an additional way to verify that the energy scale has been converted correctly.

According to equation 4.6, assuming a total timing resolution of $\sim 100$ ps (verifiable through the width of the measured 'photon peak' in Figure 4.11), the energy resolution at the $55 \mathrm{eV}$ kinetic energy of the first order Fermi level (with the total flight path length of $0.586 \mathrm{~m}$ ) is only $82 \mathrm{meV}$ at best. To achieve better energy resolution of the Fermi edge, the photoelectrons must be retarded with the electrostatic lens column. Once voltages are 
applied however, one can no longer use equation 4.1 for the time to energy conversion. However, it still holds that $E_{K} \propto 1 / t^{2} .{ }^{123}$ In a sense, by applying voltages to the lens column electrodes, the photoelectrons acquire an effective mass, $m^{*} \neq m_{e}$, to be used in the time to energy conversion. We already saw how the Au spectra contains multiple points of known energy separation, allowing one to easily fit for the correct effective mass. In the absence of these features, one can take many spectra tracking a single feature while scanning photon energy to properly fit the effective mass.

By setting the photon energy down to $h \nu=30 \mathrm{eV}$, and applying a set of voltages (or a 'lens table') to the lens column to give an average flight energy of about $7 \mathrm{eV}$, the spectrum of Figure 4.14 was acquired and converted to energy. The measured width of the resulting Fermi edge is $\sim 31 \mathrm{meV}$. This represents a convolution of a number of sources of energy broadening, including sample temperature, photon energy resolution, and the resolution of the actual TOF analyzer, where

$$
\Delta E_{\text {Total Measured }}=\sqrt{\Delta E_{\text {photon }}^{2}+\Delta E_{\text {temp }}^{2}+\Delta E_{\mathrm{TOF}}^{2}} \cdot
$$

The temperature of the Au had drifted up to about $50 \mathrm{~K}$ making $\Delta E_{\mathrm{temp}}=17 \mathrm{meV}$, and the beamline settings gave $\Delta E_{\text {photon }}=23 \mathrm{meV}$. Using these values, an effective TOF energy resolution of about $10 \pm 5 \mathrm{meV}$ can be extracted. Achieving lower sample temperature and better beamline resolutions would provide a more precise way of determining the TOF contribution. Although this resolution is excellent for a spin-resolved experiment, it is worse than the theoretical resolution of $<1 \mathrm{meV}$ based on the path length, timing resolution, and flight energy. The degraded energy resolution achieved in this experiment is likely caused by the large beamspot size.

In any case, the results of this section gave confidence that the basic experimental layout of Figure 4.10, including the detector scheme, timing electronics, and lens column plan, provide a good basis for a high-quality TOF photoelectron analyzer. The next step would be to develop a more complete electrostatic lens system. 


\subsubsection{A few remarks on TOF overlap}

Now that we have seen some actual TOF spectra, we can look a little more closely at the concept of spectral overlap (introduced in section 4.1), where fast electrons from a given light pulse overlap with slower electrons from earlier light pulses. We will also consider the resulting restrictions on excitation source rep-rates and photoelectron flight energies. We can use the present example of the above Au spectrum taken with $h \nu=60 \mathrm{eV}$, a $0.586 \mathrm{~m}$ flight path, and $328 \mathrm{~ns}$ between light pulses. At first one may think that this problem wouldn't allow any photoelectron spectrum to ever be successful, even without any retardation voltages. Ignoring the higher harmonic light, the fastest photoelectrons will be those from $\mathrm{E}_{F}$ with a kinetic energy of about $55 \mathrm{eV}$, but there will be photoemission all the way down to extremely low kinetic energy, essentially to zero. In fact, due to the secondaries piling up at low kinetic energy, we expect there to be quite a lot of photoelectrons at these low energies. These ultra-low kinetic energy electrons will take a very long time to reach the detector; taken to the extreme, a $0 \mathrm{eV}$ photoelectron will take infinite time to reach the detector. Clearly, there must be TOF spectral overlap no matter what, unless we reduce the light source rep-rate to 0 . With closer inspection, it turns out that the non-linear bin widths (which leads to the need for normalization through equation 4.10) saves us, and allows time spectra such as Figure 4.11 to be clear and not a hopelessly cluttered tangle.

Let us illustrate this through the specific example. From equation 4.1, the fastest photoelectrons $(55 \mathrm{eV})$ have a flight time of $133.322 \mathrm{~ns}$. This time bin will collect counts from $55 \mathrm{eV}$ electrons, but also from electrons which take $133.322+328$ ns to reach the detector (from the previous pulse). Again, from equation 4.1 we see that these electrons will have a kinetic energy of $\sim 4.59 \mathrm{eV}$. Since we know that there will be photoemission at $4.59 \mathrm{eV}$ (and below), there must in fact be spectral overlap. However, let us invert equation 4.10 to see how a spectrum in energy is measured in time bins. This way we have

$$
C(\tau)=I\left(E_{\tau}\right) \times \frac{2 E_{\tau}}{\tau} \Delta \tau
$$

Let's simplify things and assume that the energy spectrum emitted from the sample is constant, $I\left(E_{\tau}\right)=I$, for all $E<55 \mathrm{eV}$. The number of counts in the $\mathrm{t}=133.322 \mathrm{~ns}$ channel 
of the time spectrum will be a sum of counts due to photoelectrons arriving at $133.322 \mathrm{~ns}$ $(55 \mathrm{eV}), 133.322+328 \mathrm{~ns}(4.59 \mathrm{eV}), 133.322+2 \times 328 \mathrm{~ns}(1.57 \mathrm{eV})$, etc. The number of counts due to the $55 \mathrm{eV}$ photoelectrons is

$$
C(133.322)=I \times \frac{2 \times 55}{133.322} 0.004883 \approx 4.03 \times 10^{-3} \times I
$$

where we have used the fact that each time bin is $0.004883 \mathrm{~ns}$ wide. The number of counts due to the $4.59 \mathrm{eV}$ electrons is

$$
C(461.322)=I \times \frac{2 \times 4.59}{461.322} 0.004883 \approx 9.72 \times 10^{-5} \times I .
$$

Clearly, the contribution from the $1.57 \mathrm{eV}$ electrons will be even less, and so forth. Thus the counts in this time channel is in fact dominated by the actual $55 \mathrm{eV}$ signal, with only insignificant contributions from overlapping electrons. In general, the ratio of counts in a given time bin, $\tau$, due to electrons of energy $E_{\tau}$ to those due to electrons of energy $E_{\tau+T}$, where $T$ is the time period of the excitation source, is given by

$$
R=\frac{C(\tau)}{C(\tau+T)}=\frac{I\left(E_{\tau}\right)}{I\left(E_{\tau+T}\right)} \times\left(\frac{E_{\tau}}{E_{\tau+T}}\right)^{3 / 2}
$$

where we have used equations 4.13 and 4.1. This ratio should be much greater than 1 if the spectrum is needed to be 'clean' and not distorted due to the overlapping low energy electrons. For the numbers in the above experiment, we have $R \approx 41$, suggesting a fairly clean spectrum of the near $\mathrm{E}_{F}$ electrons.

While we are here, we can inspect what would happen if the same experiment as above was performed during multi-bunch mode, where $T$ is only 2 ns. The shorter period now means that the $55 \mathrm{eV}$ electrons at $\mathrm{E}_{F}$ are overlapped with electrons of $53.39 \mathrm{eV}$ kinetic energy. From equation 4.16, we have a corresponding count ratio of $R \approx 1$ suggesting a very 'unclean' spectrum where the spectral weight of the $55 \mathrm{eV}$ electrons is hopelessly mixed with that of lower energy electrons. A meaningful energy spectrum cannot be extracted from the resulting time spectrum.

We can also inspect what would happen if we decided (during 2-bunch mode) to retard the photoelectrons at $\mathrm{E}_{F}$ from $55 \mathrm{eV}$ all the way down to $4 \mathrm{eV}$ to greatly increase the energy resolution. Now, the $\mathrm{E}_{F}$ photoelectrons at $4 \mathrm{eV}$ arrive at the detector after 494.37 
$\mathrm{ns}$, and are overlapped by electrons of $1.45 \mathrm{eV}$. Equation 4.16 gives $R \approx 4.6$. Although the true spectrum at $4 \mathrm{eV}$ may be qualitatively recognizable, it will be difficult to extract meaningful quantitative data about the photoelectrons at $\mathrm{E}_{F}$ due to this overlap. A longer time window between light pulses would be required to quantitatively study photoelectrons at these flight energies.

In fact, significant contributions from overlapping slower electrons is already evident at $7 \mathrm{eV}$ flight energies, as can be seen in the Fermi edge spectrum shown in Figure 4.14. The bottom of the y-axis is the true zero of the plot; it is clear that the observed Fermi edge is on top of a substantial background as the edge does not drop down to zero as expected. This background level is larger than the background visible above the Fermi edge in the higher energy spectrum in Figure 4.13(b), which is due to the higher harmonic light. This increased background is due to contributions of slower electrons from earlier pulses. While this does not effect the ability to qualitatively and pseudo-quantitatively interpret the spectrum in this case (the shape of the overlapping contribution is a fairly level background), it illustrates that overlapping effects are already beginning to degrade the accuracy of the spectrum at these flight energies.

These examples help to illustrate the restrictions placed on both the light pulse period and the range of photoelectron flight energies present, and the inter-dependence of these restrictions. These are important concepts and considerations for the design of any TOF experiment.

\subsection{Electrostatic lens system}

\subsubsection{General design considerations}

The previous sections have illustrated a number of important concepts related to the TOF technique which guided the design of the current TOF analyzer. The goal is to produce a system capable of providing simultaneous high collection efficiency and high energy resolution for spin-ARPES experiments, compatible with a wide range of possible light 


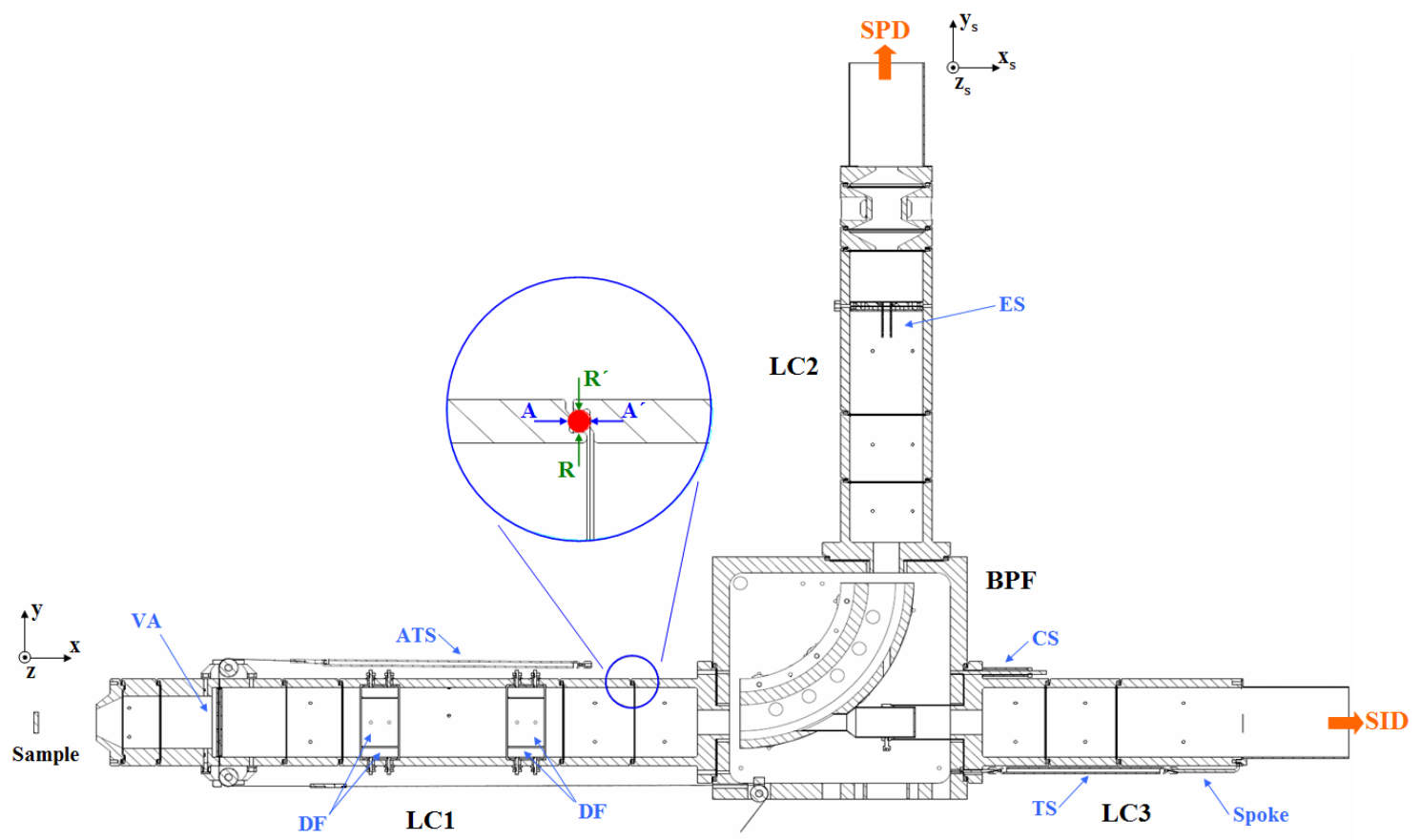

Figure 4.15. Sectioned view of the TOF Lens System schematic showing the basic design concepts. The three lens columns are labeled LC1, LC2, and LC3. BPF: bandpass filter; VA: variable aperture; ES: exit slit; ATS: aperture tension spring; TS: tension spring; CS: compression spring; DF: deflectors; SID: spin integrated detector; SPD: spin polarimeter detector. Details in text.

sources and corresponding photon energies. Achieving this goal requires a well designed, multi-element lens system for flexible retardation ratios and focal conditions. A sectioned view of the final design schematics for accomplishing these goals is shown in Figure 4.15. Specifics of the electron-optical properties of the lens system were designed by Gennadi Lebedev of the ALS, and a brief description has been published in ref. 139.

The TOF lens system is comprised of three lens columns, LC1, LC2, and LC3, enabling two main modes of operation. In one mode, photoelectrons from the sample are accepted into the front of LC1 and continue through into LC3 and then into a spin integrating detector (SID). Currently, the SID is the same Hamamatsu F4655-12 MCP detector assembly as used in the prototype TOF analyzer. In the second mode, photoelectrons passing through LC1 are deflected around a $90^{\circ}$ bandpass filter (BPF), down LC2 and into a spin polarimeter detector (SPD) which provides high efficiency spin-resolution. The SPD is described in the next chapter. 
Although other light sources are envisioned, the primary intended source is the ALS 2bunch mode, and so the system must meet the restrictions discussed above in regards to time spectrum overlap. For the highest resolutions, we saw in section 4.4.5 that difficulties due to overlap will occur. For the spin-ARPES experiments of primary concern, the electronic structure near $\mathrm{E}_{F}$ is the most interesting - the lower kinetic energy (higher binding energy) electrons are of little interest, and ideally could be removed from the spectrum to prevent their overlapping with the near $\mathrm{E}_{F}$ photoelectrons. The electrostatic BPF was introduced into the design to be able to remove the unwanted portions of the emitted spectrum to allow the most interesting electrons to be studied with high resolution. As illustrated by the SDA discussed in section 2.7.1, nested hemispherical electrodes spatially disperse an electron beam as a function of kinetic energy. Therefore, a suitably designed section of spherical electrodes, followed by an exit slit (ES), removes unwanted portions of the spectrum, allowing only those within the desired range to pass. This, in turn, allows spectra to be taken at lower flight energies for high energy resolution.

The $90^{\circ}$ BPF brings additional advantages for spin-ARPES. Typically, a spinpolarimeter can only analyze the spin polarization with respect to one or two axes fixed by the polarimeter's physical orientation with respect to the sample being studied. In the case of our $\mathrm{SPD}$, spin can be resolved along directions in the plane formed by the $x_{s}$ and $z_{s}$ axes of the SPD, as illustrated in Figure 4.15. Because the BPF is electrostatic, photoelectron spin direction is constant around the BPF; in other words, a spin initially oriented along the sample's $x$ axis stays in that direction around the BPF and is measured as oriented along the SPD's $x_{s}$ axis. The SPD is sensitive to spin in the $x_{s}$ and $z_{s}$ directions, and so as drawn in the figure, the SPD can analyze spin along the sample's $x$ and $z$ axes, but not the $y$ axis. If the entire TOF spectrometer is rotated $90^{\circ}$ into the page around the optical (axial) axis of LC1/LC3, the spin sensitive $x_{s}$ and $z_{s}$ axes become aligned with the sample's $x$ and $y$ axes, respectively, allowing spin resolution along the sample's $y$ axis. Exciting spin-ARPES experiments will involve a range of geometries where spin-resolution may be desired along any (or all three) axes. The $90^{\circ} \mathrm{BPF}$ gives the experimenter the flexibility to perform the 
experiment in the most suitable geometry by providing spin resolution along all 3 spatial axes.

The addition of the BPF also allows the easy addition of a second detector at the end of LC3 that can be used simply by removing the voltages from the BPF electrodes; in this case the electrons pass straight through a $1 / 2^{\prime \prime}$ diameter hole in the outer spherical electrode.. Although the unique design of the SPD provides two orders of magnitude greater efficiency than other spin polarimeters, it is still less efficient than a spin-integrated detector by about a factor of 100. Therefore, a SID attached to the end of LC3 provides the ability for fast spin-integrated measurements. It is envisioned that a spin-ARPES experiment could first begin by taking fast spin-integrated ARPES to aid in characterizing and aligning the sample. Because LC3 forms a straight path, the entire spectrum is taken at once. This is good as the data will automatically include any core levels which may be helpful in sample surface characterization. However, due to spectrum overlap issues (section 4.4.5), the data will need to be taken at higher flight energies for somewhat worse energy resolutions than possible with the BPF path. Once the surface has been characterized and aligned, and areas in k-space are identified where spin-resolution is most desired, the experimenter can easily turn on the BPF and switch to high resolution spin-ARPES. This design feature adds another level of flexibility to the spectrometer and the experiments performed with it.

\subsubsection{Magnetic shielding}

Precise control and interpretation of electron flight times through the electrostatic analyzer requires minimal residual magnetic fields. The simple expression for the cyclotron radius of an electron in a constant magnetic field, $r_{c}=\frac{m_{e} v}{e B}=\sqrt{2 m_{e} E} / q B$, shows that electron flight paths will be more sharply distorted for high magnetic fields and lower kinetic energies. The low flight energies desired for high energy resolution means that the residual magnetic fields must be kept quite low. Magnetic shielding, made of sheets of ' $\mu$-metal' surrounds the entire spectrometer to create a space of low magnetic field, free of the Earth's field as well as other laboratory sources. $\mu$-metal is an $80 \% \mathrm{Ni}$ and $20 \% \mathrm{Fe}$ soft-ferromagnetic alloy with extremely high magnetic permeabilities (therefore the $\mu$-metal 


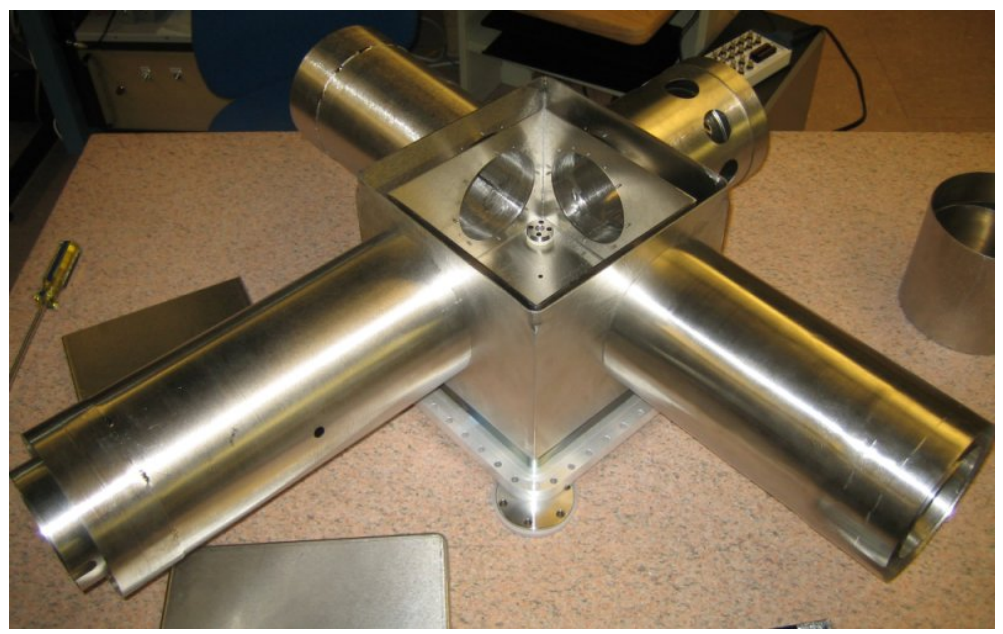

Figure 4.16. Assembled double layer $\mu$-metal shield (without 'lids' on box section for better view). Shield was assembled on its own to allow measurement of residual fields before final assembly around actual lens system.

name) that 'soak-up' magnetic fields lines like a sponge, diverting them around areas which it encloses. Two $0.040^{\prime \prime}$ thick layers separated by $3 / 4^{\prime \prime}$ surround the entire spectrometer for enhanced shielding with as little weight as possible.

The magnetic shielding introduces added complexity to the entire design. The strange physical shape of the TOF analyzer means strangely shaped $\mu$-metal which must be made from separate pieces, and then joined at different steps of analyzer assembly. All penetrations through the shielding, for electrical leads, motion feedthrus, etc, must be kept to a minimum and be as small as possible. The joints from section to section must be well designed for rigid, strong mechanical contact so that the joints don't 'leak' field lines. The shielding also must mate with shielding surrounding the analysis chamber to form a continuous shield around the sample, as well. Magnetic shielding can only protect the analyzer from external fields; it can do nothing about fields from magnetized components inside the shielding. Therefore, all analyzer components inside the shielding must be fabricated from non-magnetic parts to avoid the possibility entirely.

A test assembly of the double-layered shielding is shown in Figure 4.16, with the two 'lids' of the shielding which surround the central 'box' electrode removed. A clever slotlocking design for rigidly bolting the column shields to the box shield allows strong connec- 


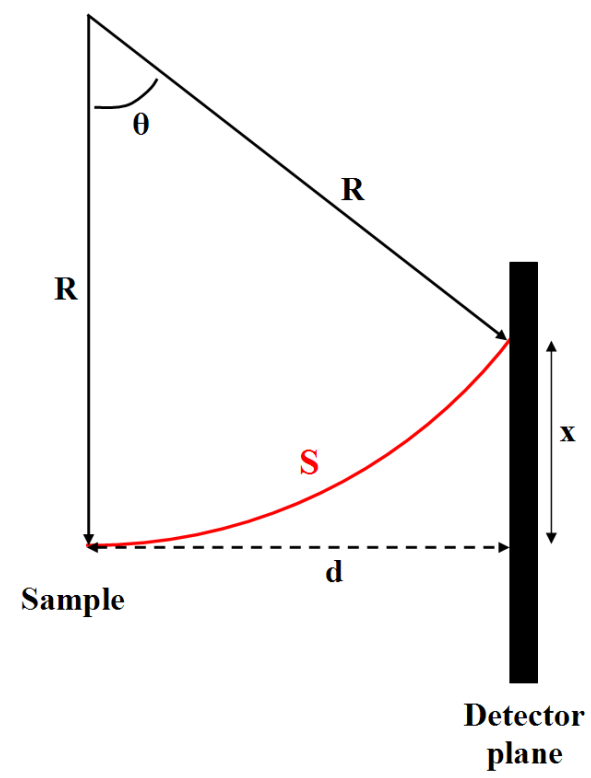

Figure 4.17. Path of photoelectron from sample to detector along arc $S$. See text for details.

tions with easy installation, even when inside the vacuum chamber. Testing the magnetic fields within the shielding at this stage showed residual fields everywhere below $1 \mathrm{mG}$, and mostly around $0.1 \mathrm{mG}$. The cyclotron radius of a $1 \mathrm{eV}$ electron in a field of $0.1 \mathrm{mG}$ is over $337 \mathrm{~m}$, and so can be considered a negligible path length change over a $1 \mathrm{~m}$ flight path.

A more thorough analysis can be taken as follows. Figure 4.17 illustrates the path of a photoelectron from the sample to the detector plane in the presence of a constant $\mathbf{B}$-field directed out of the plane. Instead of taking the direct path of distance $d$, the photoelectron will take the path of arc length $S$. The radius of curvature is the cyclotron radius given by

$$
R=\frac{m v}{e B}=\frac{\sqrt{2 m E}}{e B} .
$$

It is straightforward to calculate the distance, $x$, that an electron is displaced along the detector. For a given detector distance, $d$, and residual magnetic field, $B$, it is clear that larger displacements will result for lower kinetic energies. Solving in the case of extremely low kinetic energy, for a residual magnetic field of $0.1 \mathrm{mG}\left(10^{-8} \mathrm{~T}\right)$, a distance of $d=1 \mathrm{~m}$ and electron kinetic energy of $1 \mathrm{eV}$, we have $x \approx 1.5 \mathrm{~mm}$. This is not a very large shift, and usual drift energies will be significantly higher than $1 \mathrm{eV}$.

Perhaps more interesting is investigating the effect of residual fields on our ability to 
correctly measure the flight energies. Again we will use Figure 4.17 for reference. An effect seems plausible as the total path length, $S$, depends on the cyclotron radius, $R$, which is in turn a function of flight energy. We know from section 4.1 that the measured flight energy is effected by path length differences, and so here we must inspect the effect of energy dependent path lengths! We can start by noting that

$$
S=R \cdot \theta=R \cdot \arcsin \left(\frac{d}{R}\right)
$$

We are interested in the change in path length with respect to energy, so we can take the derivative of equation 4.18 as

$$
\frac{\partial S}{\partial E}=\frac{\partial S}{\partial R} \frac{\partial R}{\partial E}
$$

Starting with the first term we have (with some patience)

$$
\frac{\partial S}{\partial R}=\arcsin \left(\frac{d}{R}\right)-\frac{d}{\sqrt{R^{2}-d^{2}}}
$$

For the fields and energies used above, we had $R \approx 337 \mathrm{~m}$, so we already know $R \gg d$. Therefore we can make the following approximations,

$$
\begin{array}{r}
\arcsin \left(\frac{d}{R}\right) \approx \frac{d}{R} \quad \text { and } \\
\frac{d}{\sqrt{R^{2}-d^{2}}}=\frac{d}{R} \frac{1}{\sqrt{1-d^{2} / R^{2}}} \approx \frac{d}{R}\left(1+\frac{d^{2}}{2 R^{2}}\right)
\end{array}
$$

to substitute into equation 4.20 to give

$$
\frac{\partial S}{\partial R} \approx-\frac{d^{3}}{2 R^{3}}
$$

Now from equation 4.17 we have

$$
\frac{\partial R}{\partial E}=\frac{\sqrt{m}}{e B \sqrt{2 E}}=\frac{R}{2 E} .
$$

Substituting equations 4.21 and 4.22 into equation 4.19 , we finally have

$$
\frac{\partial S}{\partial E} \approx-\frac{d^{3}}{4 E R^{2}} .
$$

Using equation 4.17 to substitute for $R$ in the term outside the parenthesis gives

$$
\frac{\partial S}{\partial E} \approx-\frac{e^{2} B^{2} d^{3}}{8 m E^{2}}
$$


Equation 4.24 shows that the path length dependence on energy scales quadratically with field strength and inversely proportional to the square of the flight energy. So it seems the tolerable residual magnetic field decreases quickly with decreasing flight energies. Another interesting result from equation 4.24 is that $\partial S / \partial E$ scales as $d^{3}$ : although the longer flight distances improves ideal TOF energy resolutions, it apparently greatly increases magnetic field sensitivity. Achieving high resolution TOF analysis using low flight energies and long flight paths requires high quality magnetic shielding! In the case of $1 \mathrm{eV}$ electrons in a field strength of $0.1 \mathrm{mG}$, we have

$$
\frac{\partial S}{\partial E} \approx 2.2 \mu \mathrm{m} / \mathrm{eV}
$$

In the absence of magnetic field, we use equation 4.1 (with an effective mass in the case of applied electrostatic fields) to convert the time spectrum to an energy spectrum. The resulting error, $\Delta E$, in the measured energy separation of nearby points (separated by $\delta E$ ) due to energy dependent path length differences is given by

$$
\frac{\Delta E}{E}=\frac{2 \Delta S}{S} \approx \frac{2}{S} \frac{\partial S}{\partial E} \delta E
$$

Again, for the difficult region of $1 \mathrm{eV}$ with a residual field of $0.1 \mathrm{mG}$ and a total average path length of $1 \mathrm{~m}$, using the result of equation 4.25 gives

$$
\frac{\Delta E}{E} \approx-4.4 \times 10^{-6} \delta E / e V
$$

expressing the relative error in the converted energy scaling. This suggests that using equation 4.1 for time to energy conversion remains quite accurate even at low kinetic energies if the magnetic field is kept as low as $0.1 \mathrm{mG}$.

\subsubsection{Mechanical and material considerations}

Accurate electrostatic control of the photoelectrons depends on the precision to which the electrodes of the lens system are aligned to each other. Much effort was invested in the mechanical design and fabrication of the TOF lens system to achieve as strict alignment tolerances as reasonably possible. The design developed by Nord Andresen of the Engineering Division at LBL relied on developing three separate lens columns cantilevered 


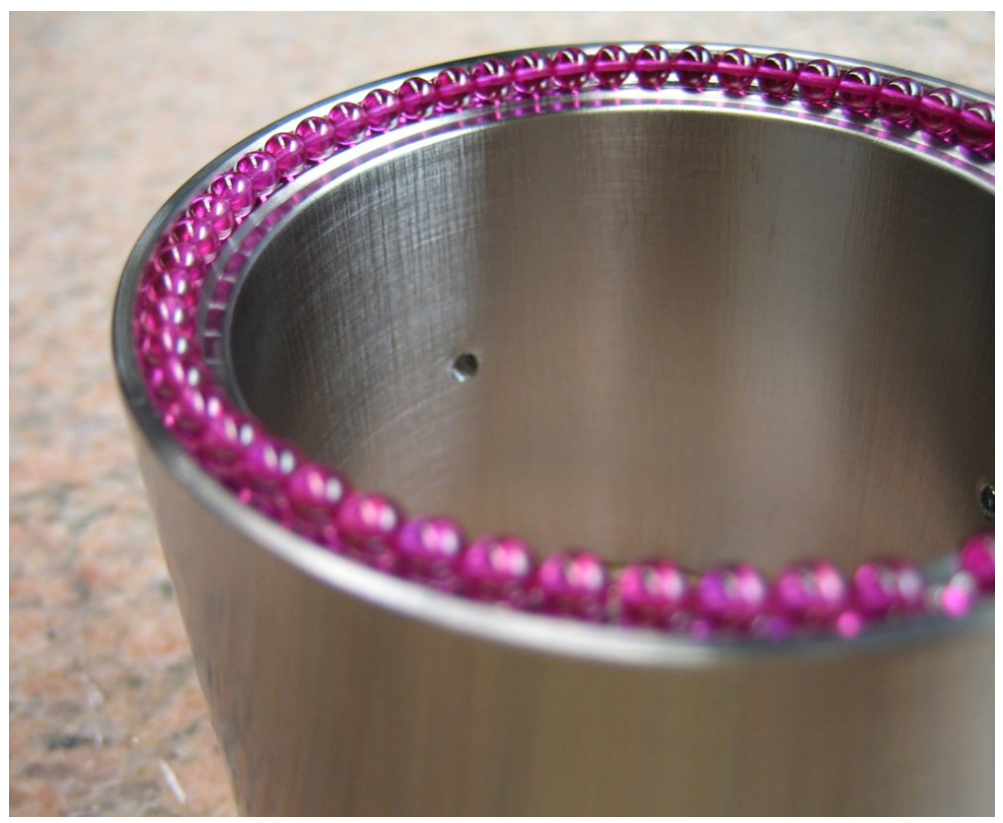

Figure 4.18. One end of a cylindrical electrode, with its bracelet of 56 ruby balls in place, contacting surfaces $A^{\prime}$ and $R^{\prime}$, as labeled in Figure 4.15.

from a central 'box' electrode which houses the $90^{\circ} \mathrm{BPF}$ electrodes. In the case of LC1, the column is quite long $(428 \mathrm{~mm})$, and the stresses on the column had to be carefully managed. Rotating the entire lens system as discussed in the previous section for accessing different spin-sensitivity directions alters these gravitation forces, requiring a very rigid construction which does not 'sag' when held in different orientations.

To achieve these goals, the design consists of isolating each cylindrical electrode in a given column from each other by a ring of 56 individual $1 / 8^{\prime \prime}$ grade 5 ruby/sapphire balls (Grade 5 balls have a diameter tolerance of 0.13 micron). To simplify assembly, each ball has a small hole drilled through the center so that they could be threaded onto a nichrome wire to form a bracelet for each electrode junction, as shown in Figure 4.18. The ruby balls not only electrically isolate each electrode, the also align each electrode to be coaxial. This can be seen in the expanded view of the first electrode joint in LC1 shown in Figure 4.15. The cross section of the rim of each electrode is designed such that they 'nest' inside each other, separated by rigid contact with the ruby ball (shown in red) on four sides. The contact along surfaces $A$ and $A^{\prime}$ align the axis of each electrode to be parallel, and the 
contact along surfaces $R$ and $R^{\prime}$ align each axis to be collinear. For high tolerances of the assembled column, the final machining on each of these surfaces were diamond turned on fully temperature controlled lathes at Lawrence Livermore National Lab. In particular, this achieved uniform diameters of the $R$ and $R^{\prime}$ surfaces to within 5 micron around their 53.975 $\mathrm{mm}$ and $60.325 \mathrm{~mm}$ respective diameters. To ensure column rigidity, the $R$ and $R^{\prime}$ surfaces were cut to diameters to ensure a slight interference fit so that there is no radial play.

Holding each lens column together is performed by 8 commercial titanium bicycle spokes, equally spaced around the column diameter, attached directly to the free end electrode of each column and attached to the fixed end electrode via stiff springs which were preloaded to 7 lbs tension each (spoke and spring shown for only one location along LC3 in Figure 4.15, rotated $22.5^{\circ}$ from true position so that it would show in the drawing). This loads each ball to $1 \mathrm{lb}$. This amount of total tension keeps the entire electrode stack rigid and straight even when cantilevered horizontally. Each lens column is mated to the central 'box' electrode via similar mating surfaces separated by a ring of 81 ruby balls. The columns are each held in place against the box with 8 threaded rods and compression springs preloaded to $10 \mathrm{lbs}$. each (again, $1 \mathrm{lb}$. per ball). Both tension and compression springs were custom wound from 'Elgiloy MP35N', a nearly non-magnetic Ni-Co-Cr-Mo alloy with high tensile strength.

The total compression forces between the ruby balls and each electrode surfaces $A$ and $A^{\prime}$, due to the required preloading plus gravitational loading, must be dealt with to avoid damaging the carefully machined electrode surfaces. To lessen the large pressures from point-contact between the balls and a flat surface, surfaces $A$ and $A^{\prime}$ are actually machined as toroidal surfaces to increase the contact area with the ruby balls. Even so, the electrodes must be made out of a very strong material able to handle large pressures without yielding. Titanium was chosen as the electrode material due to its high strength, its light weight to reduce the required preloading and total pressures, and due to its non-magnetic properties. For the same reasons of it being light weight and non-magnetic, and the desire to have all electron-optical surfaces made of the same material to avoid contact potential issues, all electrode pieces are titanium, as are the vast majority of the remaining pieces, as well. While titanium provides ideal properties, its use made the total lens system quite expensive. 
Despite the large efforts for tight machining tolerances, one parameter ended up being the most critical. The opposing axial mating surfaces on each given electrode were slightly non-parallel so that as they were stacked to form a column, there was a tiny 'kink' at each joint. For the longer columns, LC1 and LC2, a tiny kink at the base leads to a larger displacement of the electrodes at the end. Careful measurements of the slight angle between the axial surfaces of each electrode allowed finding the best rotational alignment of each cylinder with respect to the others such that each kink mostly opposed the last. This resulted in minimal misalignment of the total column. Each completed column was then vertically mounted on a high precision Bridgeport vertical milling machine spindle with a custom adjustable mounting block. The column could be adjusted to have zero run-out at the two ends of the column as the spindle rotated, and then a careful measurement could be made to find the maximum run-out along the length of the column in between. In other words, each completed column was still slightly 'banana' shaped (as is any realistic column). The banana was attached to the spindle so that it would rotate along the axis connecting the two ends of the banana, and then the middle was measured to inspect how 'bowed' it was. For LC1, LC2, and LC3, the resulting maximum displacements from the central axis were $32 \mu \mathrm{m}, 8 \mu \mathrm{m}$, and $12 \mu \mathrm{m}$, respectively. After the 'correct' rotational alignment was decided, the electrodes were marked, disassembled, and UHV cleaned for final assembly.

\subsubsection{Assembly and inspection}

To reduce secondary electron emission and minimize work function irregularities, the electron-optical surfaces of every electrode were coated with a fine colloidal graphite layer before final assembly, applied with an air brush. The cylindrical electrodes were then vacuum baked to help ensure the graphite layer adhered to the surfaces and aid in complete

degassing of any remaining volatile solvent. The resulting finish was high-quality by eye, as shown in the photograph of the last electrode of LC2 on the left of Figure 4.19. After coating, the cylindrical electrodes were stacked to form the three lens columns once again. Each was assembled in a special stand so that a counter weight could be attached to the springs from below to allow constant preloading of each spring. The spherical BPF electrodes were 

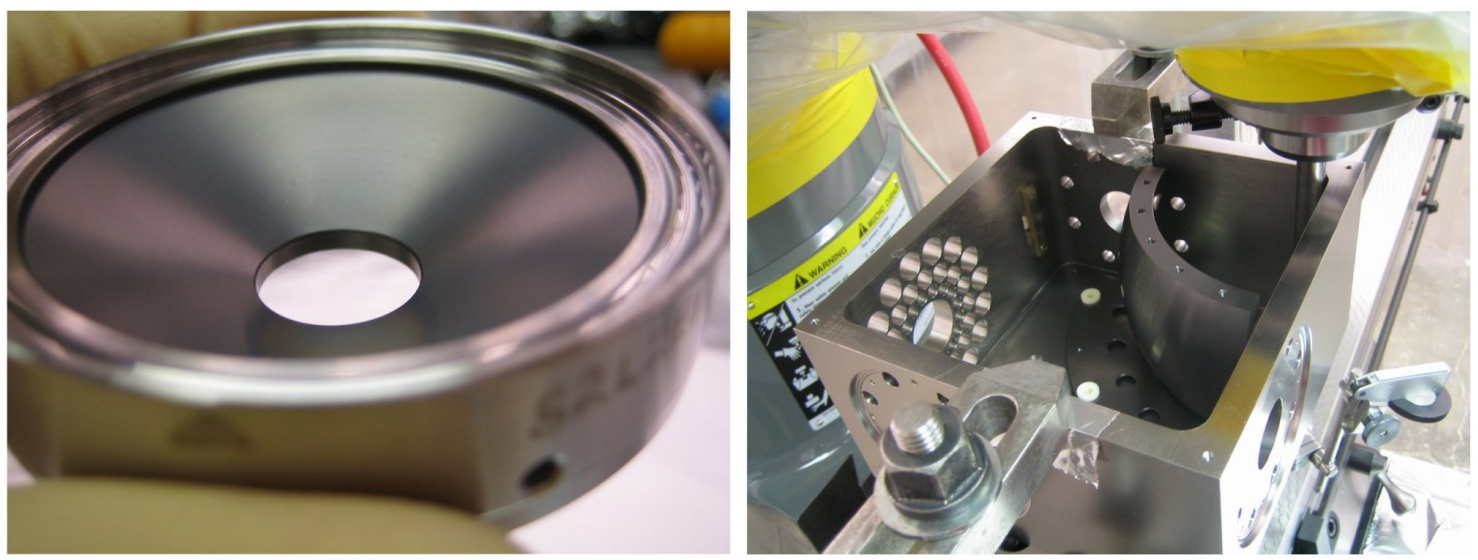

Figure 4.19. Left: Graphite coated inner surface of last cylindrical electrode of LC2. Right: Installation of inner spherical electrode into 'box' electrode. Box is bolted to the table of a clean milling machine and a circular reference surface in box is aligned to the spindle. Circular reference surfaces on spherical electrodes are then aligned to the spindle.

also coated, baked, and assembled into the 'box' electrode. Precise concentric alignment of the electrodes required again using the mill spindle to ensure each electrode was concentric with a circular reference surface machined into the box. A photograph of the inner spherical electrode being installed is shown on the right of Figure 4.19.

The $\mathrm{BPF}$ requires an exit slit in LC2 (as shown in Figure 4.15) to define the range of energies passed. For added flexibility, a variable exit slit was included to allow run-time setting of optimum slit size for resolution and count rate. The exit slit is two 'paddles', $1^{\prime \prime}$ long along the optical axis of LC2, with 29 saw-tooth ridges for minimizing the transmission of reflected electrons. These paddles are mounted on a high-precision flexure mount for parallel opening and closing, spring loaded with a drive screw for control, shown in the photo on the left of Figure 4.20. The drive screw terminates in the Al-Si-bronze 'star-wheel' visible in the photo. A rotary motion feedthru with a mating attachment opens/closes the slit by turning the star-wheel. The junction between the rotary feedthru and the star-wheel gives slight free 'play' so that after setting the size of the slit, the feedthru can be turned back a couple degrees releasing any physical contact between the feedthru and the star-wheel. This ensures that the feedthru does not place any external stresses on the column during actual measurement A photo of the completed unit before installation into its cylindrical electrode is shown in the right of Figure 4.20. 

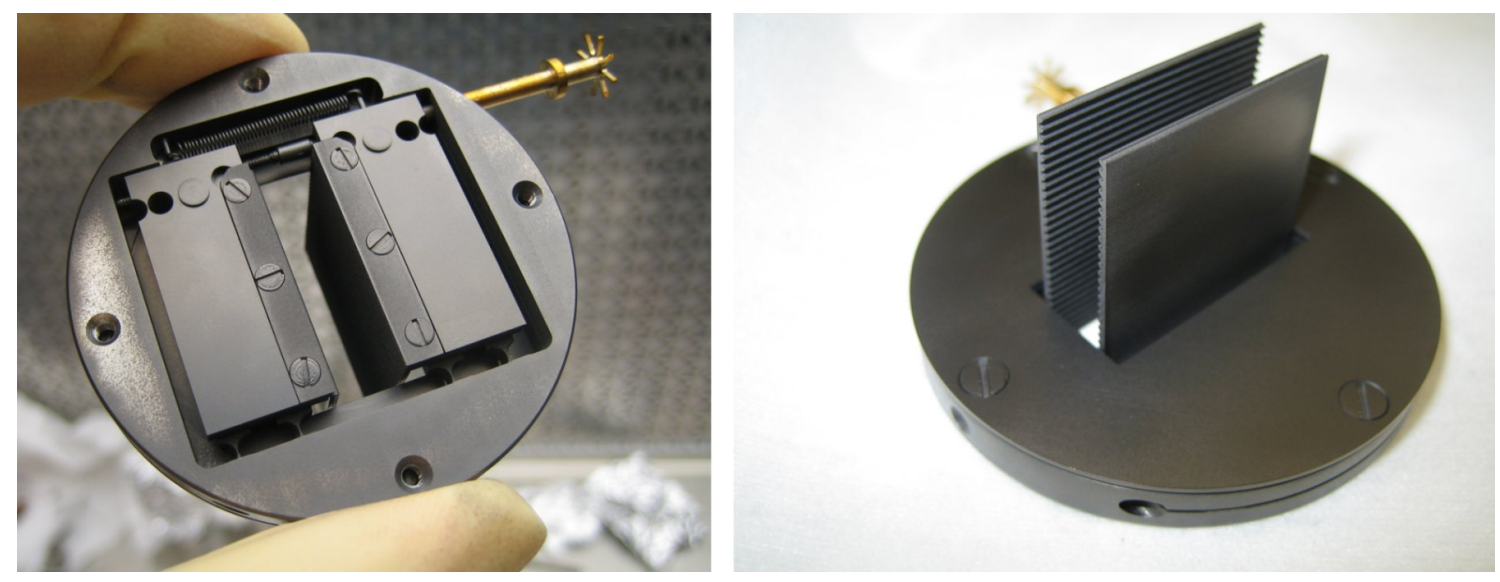

Figure 4.20. The BPF exit slit in LC2. Left: inner view of flexure mounting of the slit paddles. Right: upstream view of the completed unit. Ridges along paddles help keep transmission of electrons scattering off the paddles low.

The variable aperture near the entrance of LC1 is formed by a long metal foil band with a series of 4 holes of increasing diameter. This band passes through one of the front electrodes of LC1, and around two exterior pulleys, as shown in Figure 4.15. The band is spring loaded on one side and can be pulled with a translational feedthru on the other side to move the desired size aperture into place like a clothesline. The same design was used in the prototype, and it can be viewed in the photos of both the prototype and full TOF analyzers in Figures 4.8 and 4.21, respectively. The run-time selectable aperture size gives the flexibility of deciding on an optimal compromise between count rate and angular resolution for the experiment at hand.

After the BPF was assembled into its magnetic shielding and vacuum housing, this subassembly was mounted to a large rotary table that allowed positioning the central assembly in any orientation. This was very helpful, as each completed lens column is best installed onto the box electrode while vertical. After each lens column was installed, the assembly could be rotated to allow the next column to be installed vertically.

This rotary mounting also allowed convenient measurement of the resulting sag of each column. The position of a given column with respect to the center of the BPF could be accurately measured when the column was horizontal. The assembly was then rotated $180^{\circ}$ so that the column was horizontal in the opposite direction, and the position measurement 


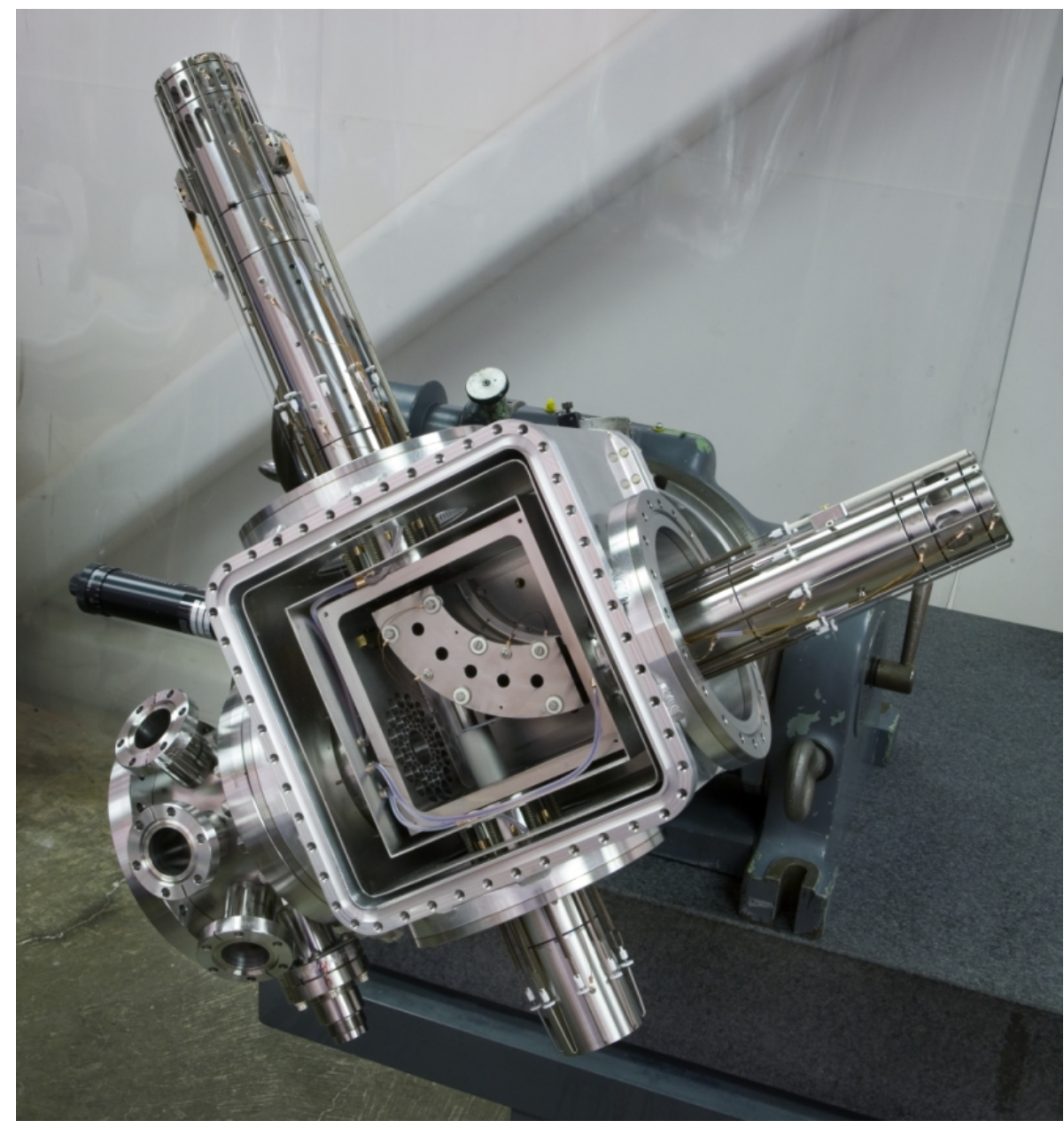

Figure 4.21. TOF lens system assembly. Parts of vacuum chamber, magnetic shielding, etc, removed for better view.

was made again. The difference in the two measurements would then represent twice the 'sag' from a vertical orientation to a horizontal orientation. The measured amounts of sag for $\mathrm{LC} 1, \mathrm{LC} 2$, and $\mathrm{LC} 3$ were $34 \mu \mathrm{m}, 9.5 \mu \mathrm{m}$, and $3 \mu$, respectively.

The assembled TOF lens system is shown in Figure 4.21. It is held by the rotary table behind it. The exterior of the variable aperture band can be seen near the top of LC1. Electrical leads can also be seen attached to each electrode. Each lead travels down the column, around the central 'box' electrode, and to a pin on one of three electrical feedthrus attached to the vacuum housing directly opposite LC2 (lower left of photo, with the multiple 2-3/4" flanges). This chamber section provides multiple flanges for the electrical feedthru's, additional pumping, and other unforeseen future requirements. To complete the assembly, 
a plate is attached to close off the central box electrode, and then the two magnetic shield lids are installed to complete the shielding around the BPF. Two $\mu$-metal cylinders are then installed around each lens column and rigidly bolted to the central box shielding. The main vacuum flange capping the central vacuum chamber is then bolted on, and vacuum spool pieces are installed around each lens column section to complete assembly.

This complete lens system is thus independent of detector assemblies. Detectors with independent electrical feedthrus, $\mu$-metal, and vacuum housings can be bolted onto the TOF lens systems in a 'plug-and-play' fashion. The primary plan calls for the simple Hamamatsu F4655-12 MCP detector assembly to be the detector behind LC3, and the custom SPD to be the detector behind LC2. However, if desired, the arrangement can be easily reversed, or completely different detectors can be used instead. Initial results with the primary detector arrangement will be discussed in Chapter 6 .

\subsection{Summary}

The SDA with multichannel detection has dominated the field of ARPES due to its reliability and efficiency. Coupling a spin-polarimeter or other single channel detector to an SDA does not allow the advantage of parallel detection, adding inefficiency to an already difficult experiment. The TOF technique is a well established and highly efficient scheme allowing parallel energy detection with a single channel detector. The custom TOF lens system developed here for the spin-TOF spectrometer will aid in enabling more efficient

and higher resolution spin-resolved ARPES experiments to be performed than is possible with the standard SDA. 


\section{Chapter 5}

\section{Spin Polarimetry}

The status of the polarization detectors, however, is far from being satisfactory. Though some important improvements have been made, and some are foreseeable, [...] the detectors are still the weak point in the field. The polarization sensitivity is not better than $30 \%$ and we still lose many hundreds of electrons for each one detected. Some new ideas shall be welcome...

- J. Kirschner ${ }^{16}$

\subsection{Spin Polarization}

The concept of spin polarization is straightforward. A 'beam' or ensemble of electrons is polarized if there are unequal numbers in the two possible spin states ('up' or 'down') with respect to some spatial direction. If every electron in the ensemble is in one or the other, the electrons are totally polarized, or have a polarization (absolute value) of 1 . If there are exactly the same number of both, they are unpolarized, or have a polarization of 0 along that direction. It is quite possible to have both spin states somewhat populated, but have more of one than the other; in this case the electrons are partially polarized with a polarization between 0 and 1. It was shown in Chapter 3 that measuring the polarization of the ensemble of photoelectrons as a function of energy and momentum can give powerful information about the sample's electronic structure. Unfortunately, although the concept of spin polarization is straightforward, we will see that measuring it is not. 
The classic texts of Kessler ${ }^{140}$ and Kirschner ${ }^{95}$ are excellent references of spin polarization. A brief summary of their general treatments of polarization formalism in this section will be worthwhile for understanding the various polarimetry techniques in the following sections.

In Chapter 1 the spin operator, $\mathbf{S}$, was introduced. From the spin angular momentum commutation relations, we can represent the vector spin operator in matrix form with the vector of Pauli matrices, $\sigma$, as

$$
\mathbf{S}=\frac{\hbar}{2} \boldsymbol{\sigma}
$$

It is customary to use the $S_{z}$ basis such that the z-component of the Pauli matrices is diagonalized, giving

$$
\sigma_{z}=\left(\begin{array}{cc}
1 & 0 \\
0 & -1
\end{array}\right) \quad \sigma_{x}=\left(\begin{array}{cc}
0 & 1 \\
1 & 0
\end{array}\right) \quad \sigma_{y}=\left(\begin{array}{cc}
0 & -i \\
i & 0
\end{array}\right) .
$$

The two component spin functions introduced in Chapter $1, \chi$, take the form of $\left(\begin{array}{l}a_{1} \\ a_{2}\end{array}\right)$. In this basis, $\left(\begin{array}{l}1 \\ 0\end{array}\right)$ and $\left(\begin{array}{l}0 \\ 1\end{array}\right)$ are eigenvectors of $\sigma_{z}$ with eigenvalues +1 and -1 , respectively.

The polarization of state $|\chi\rangle$ with respect to a given axis, such as the z-axis, can be written

$$
P_{z}=\frac{\left\langle\chi\left|\sigma_{z}\right| \chi\right\rangle}{\langle\chi \mid \chi\rangle}
$$

where the denominator is necessary if the state $|\chi\rangle$ is not normalized. An alternate way to express the polarization is

$$
P_{z}=\frac{N_{\uparrow}-N_{\downarrow}}{N_{\uparrow}+N_{\downarrow}}
$$

where $N_{\uparrow}\left(N_{\downarrow}\right)$ is the number of electrons counted with $S_{z}=+\hbar / 2\left(S_{z}=-\hbar / 2\right)$. Thus, for a group of electrons in the spin state $\chi=|\uparrow\rangle=\left(\begin{array}{l}1 \\ 0\end{array}\right)$, equation 5.3 gives $P_{z}=1$, meaning they are fully polarized along the z-axis and will all have an $S_{z}$ value of $+\hbar / 2$. Equation 5.3 used with respect to the $x$ and $y$ axis, however, will give $P_{x}=P_{y}=0$, meaning they are unpolarized along these axes.

The full polarization vector is given by

$$
\mathbf{P}=P_{x} \hat{x}+P_{y} \hat{y}+P_{z} \hat{z}
$$


with its magnitude, or degree of polarization, then given by $P=\sqrt{P_{x}^{2}+P_{y}^{2}+P_{z}^{2}}$. These can be more succinctly expressed as

$$
\mathbf{P}=\frac{\langle\chi|\boldsymbol{\sigma}| \chi\rangle}{\langle\chi \mid \chi\rangle}
$$

The magnitude of polarization, $P$, for electrons in a single spin state, $|\chi\rangle$, is always 1 ; the single spin state is $100 \%$ polarized along some axis, the only question is which vector direction is it pointing along. This vector can be fully determined, but each component must be determined individually on 'fresh' electrons straight from the source. Because measurement of an electron's spin along an axis changes its state, the same electron cannot be used for successive measurements along the other axes.

The photoelectron beams of interest will typically be ensembles formed by statistical mixtures of various spin states. With a mixture of $n$ states, $\left|\chi^{(n)}\right\rangle$, the magnitude of the polarization, $P$, can now range from 1 to 0 . The polarization vector is given by an extension of equation 5.6 as

$$
\mathbf{P}=\sum_{n} \frac{\left\langle\chi^{(n)}|\boldsymbol{\sigma}| \chi^{(n)}\right\rangle}{\left\langle\chi^{(n)} \mid \chi^{(n)}\right\rangle}
$$

Let's assume from here on that the states, $\left|\chi^{(n)}\right\rangle$, are normalized. In this case, the previous equation can be written

$$
\mathbf{P}=\sum_{n} g^{(n)}\left\langle\chi^{(n)}|\boldsymbol{\sigma}| \chi^{(n)}\right\rangle
$$

where the weighting factors, $g^{(n)}$, give the relative proportions of electrons in each state $\left|\chi^{(n)}\right\rangle$ according to

$$
g^{(n)}=\frac{N^{(n)}}{\sum_{n} N^{(n)}}
$$

where we define $N^{(n)}$ to be the number of electrons in state $\left|\chi^{(n)}\right\rangle$.

To aid in describing the polarization of an electron ensemble, one can define a so-called density matrix. For a single spin state, $\left|\chi^{(n)}\right\rangle=\left(\begin{array}{l}a_{1} \\ a_{2}\end{array}\right)$ (normalized), the density matrix is

$$
\rho^{(n)}=\left|\chi^{(n)}\right\rangle\left\langle\chi^{(n)}\right|=\left(\begin{array}{c}
a_{1}^{(n)} \\
a_{2}^{(n)}
\end{array}\right) \cdot\left(a_{1}^{(n)}, a_{2}^{(n)}\right)=\left(\begin{array}{cc}
\left|a_{1}^{(n)}\right|^{2} & a_{1}^{(n)} a_{2}^{(n) *} \\
a_{1}^{(n) *} a_{2}^{(n)} & \left|a_{2}^{(n)}\right|^{2}
\end{array}\right) .
$$


Extension to the mixed ensemble is trivial, giving

$$
\rho=\sum_{n} g^{(n)} \rho^{(n)}=\sum_{n} g^{(n)}\left(\begin{array}{cc}
\left|a_{1}^{(n)}\right|^{2} & a_{1}^{(n)} a_{2}^{(n) *} \\
a_{1}^{(n) *} a_{2}^{(n)} & \left|a_{2}^{(n)}\right|^{2}
\end{array}\right) .
$$

The density matrix is a useful formalism as the polarization (or individual polarization components) can be easily extracted as

$$
\mathbf{P}=\operatorname{tr}(\rho \boldsymbol{\sigma}) \quad \text { or } \quad P_{i}=\operatorname{tr}\left(\rho \sigma_{i}\right)
$$

where $t r$ is the trace. Conversely, the density matrix can be expressed in terms of the polarization as

$$
\rho=\frac{1}{2}\left(\begin{array}{cc}
1+P & P_{x}-i P_{y} \\
P_{x}+i P_{y} & 1-P_{z}
\end{array}\right)=\frac{1}{2}(\mathbf{1}+\mathbf{P} \cdot \boldsymbol{\sigma}) .
$$

Thus we see that $\rho$ can be diagonalized by defining the z-axis along the direction of $\mathbf{P}$, such that $P=P_{z}$ and $P_{x}=P_{y}=0$, giving the simpler equation

$$
\rho=\frac{1}{2}\left(\begin{array}{cc}
1+P & 0 \\
0 & 1-P_{z}
\end{array}\right)
$$

The more intuitive meaning of the polarization can be regained by inspecting equations 5.14 and 5.11 and equating the individual matrix elements to give

$$
\frac{1}{2}(1+P)=\sum_{n} g^{(n)}\left|a_{1}^{(n)}\right|^{2} \quad \text { and } \quad \frac{1}{2}(1-P)=\sum_{n} g^{(n)}\left|a_{2}^{(n)}\right|^{2} .
$$

It should be recognized that $\sum_{n} g^{(n)}\left|a_{1}^{(n)}\right|^{2}$ is simply the probability that a measurement on the ensemble of spin along the z-axis will give $+\hbar / 2$ (spin-up), and that $\sum_{n} g^{(n)}\left|a_{2}^{(n)}\right|^{2}$ is that of a measurement of $-\hbar / 2$ (spin-down). If $N_{\uparrow(\downarrow)}$ are the number of measurements of $+(-) \hbar / 2$ and $N_{\uparrow}+N_{\downarrow}$ are the total number of measurements, then these probabilities can be written as $N_{\uparrow} /\left(N_{\uparrow}+N_{\downarrow}\right)$ and $N_{\downarrow} /\left(N_{\uparrow}+N_{\downarrow}\right)$, respectively. This gives us

$$
P=\frac{N_{\uparrow}-N_{\downarrow}}{N_{\uparrow}+N_{\downarrow}}
$$

which agrees perfectly with what we expected from equation 5.4 for the pure state. At least these conventions are consistent. 
An interesting point can be made by investigating a little further. If an ensemble were $100 \%$ polarized spin-up along the z-axis $(P=1)$, equation 5.14 gives us

$$
\rho=\left(\begin{array}{ll}
1 & 0 \\
0 & 0
\end{array}\right)
$$

while an unpolarized ensemble $(P=0)$ gives us

$$
\rho=\left(\begin{array}{cc}
1 / 2 & 0 \\
0 & 1 / 2
\end{array}\right)
$$

By inspection, this means one can re-write equation 5.14 as

$$
\rho=P\left(\begin{array}{cc}
1 & 0 \\
0 & 0
\end{array}\right)+(1-P)\left(\begin{array}{cc}
1 / 2 & 0 \\
0 & 1 / 2
\end{array}\right)
$$

illustrating that any ensemble with polarization $P$, made up of $N$ total electrons, can be thought of as a mixture of $P \times N$ totally polarized electrons and $(1-P) \times N$ unpolarized electrons.

For example, consider an ensemble of 10 electrons in which 7 are 'spin-up' along the z-axis, and 3 are 'spin-down'. Equation 5.16 gives the polarization to be $P=0.4$, meaning the ensemble can be thought of as an ensemble of 4 totally polarized electrons mixed with an ensemble of 6 unpolarized electrons.

In a spin-resolved photoemission experiment, one measures the polarization of the photoelectrons as a function of energy (and momentum for ARPES). What one really wants, is to decompose each measured photoelectron spectrum into two separate spin-spectra: one spectrum of only 'spin-up' photoelectrons, and one spectrum of only 'spin-down' electrons. From the above discussion and either equation 5.14 or 5.16 , it is clear that the number, or intensity, of photoelectrons measured at $E$ and $\mathbf{k}$ with 'spin-up' will then be given by

$$
I_{\uparrow}(E, \mathbf{k})=\frac{1}{2} I(E, \mathbf{k})[1+P(E, \mathbf{k})]
$$

while the intensity with 'spin-down' will be given by

$$
I_{\downarrow}(E, \mathbf{k})=\frac{1}{2} I(E, \mathbf{k})[1-P(E, \mathbf{k})]
$$


Thus, all one needs is a good method for measuring the photoelectron's energy and momentum dependent polarization, $P(E, \mathbf{k})$.

Excellent coverage of the diverse fields of creation, utilization, and measurement of electron polarization can be found in refs. 140,95,16. The focus here will be on polarization measurement techniques that have been most readily integrated into photoemission experiments.

Techniques for measuring electron spin polarization are referred to as spin polarimetry. The perfect polarimeter would function analogously to the simple optical polarization filter: $100 \%$ of electrons with spin parallel to a given axis can pass and be detected, while those with spin antiparallel are rejected. The Stern-Gerlach experiment (SGE) introduced in section 1.2.1 provides this type of functionality by completely splitting particle beams depending on their total magnetic moments, of which spin is a component. In particular, the Ag beams originally used are split depending on the spin of the outer valence electron. Unfortunately, the SGE is only effective in splitting beams of neutral particles, and cannot work with electrons, due to the additional Lorentz force that charged particles experience in a magnetic field. As originally argued by Bohr, Pauli, and Mott, the beam spreading due to the Lorentz force and realistic beam parameters required by the uncertainty principle outweigh the splitting effect due to spin. Kessler ${ }^{140}$ gives a good account of the argument, analyzing the resulting geometry of the resulting beams and showing that they are not usefully split at all. As an interesting aside, although this assertion that SGE-type separation of electrons is impossible has long been accepted at 'the textbook level', it is still occasionally revisited $^{141}$ (however, to the best of the author's knowledge, it has never been experimentally realized).

Spin polarimeters must then rely on other techniques, which so far are always orders of magnitude less efficient than the optical polarization filter. These techniques rely on various forms of spin-dependent scattering from assorted targets. The spin dependence in these methods originates in either (or both) of the spin dependent interactions introduced in Chapter 1: the spin-orbit and exchange interactions. The majority of current polarimeters tend to be based on the spin-orbit interaction. In particular, as it is the most firmly estab- 
lished technique, more details of spin polarimetry will be introduced through the example of Mott polarimeters.

\subsection{Spin-orbit polarimetry}

\subsubsection{Mott scattering}

Much of the existing and current spin-resolved photoemission work is done with polarimeters based on 'Mott-scattering' of the photoelectrons from heavy nuclei. The plain Coulomb potential of a nucleus is shown by the solid lines in Figure 5.1(a). A more accurate scattering potential includes the spin-orbit interaction as well to give a total scattering potential of $V=V_{C}+V_{L S}$, where from section 1.2 .3 we have $V_{L S} \propto \mathbf{L} \cdot \mathbf{S}$. The resulting spin-dependent potential is shown by the dotted curves in Figure 5.1(a). Thus a spin-up electron feels a larger (or longer range) potential when passing on the left of a nucleus (and scattering to the right), and vice versa for a spin down electron.

Although tedious, one can show that the differential cross section of such a scattering event is given by ${ }^{140}$

$$
\sigma(\theta, \phi)=\sigma_{0}(\theta)[1+S(\theta) \mathbf{P} \cdot \hat{\mathbf{n}}]
$$

where $\theta$ and $\phi$ are defined by the scattering geometry shown in Figure 5.1(b). $\sigma_{0}(\theta)$ is the cross section in the absence of spin-orbit coupling, and is dependent only on $\theta$ which introduces no spin dependence into the scattering. $S(\theta)$, called the 'Sherman function' (named for his exhaustive calculations of the function ${ }^{142}$ ) is also dependent only on $\theta$, but introduces a spin dependence into the cross section due to the $\mathbf{P} \cdot \hat{\mathbf{n}}$ term, where $\mathbf{P}$ is the polarization vector of the incident electron, and $\hat{\mathbf{n}}$ is the unit vector normal to the scattering plane defined by the incident and outgoing electron wavevectors as $\hat{n}=\frac{\mathbf{k}_{\mathbf{0}} \times \mathbf{k}^{\prime}}{\left|\mathbf{k}_{\mathbf{0}} \times \mathbf{k}^{\prime}\right|}$. Note that the direction of $\hat{\mathbf{n}}$ is parallel to $\mathbf{L}$ and thus depends of whether the electron scatters to the left or right of the nucleus. Thus we see that $\mathbf{L} \cdot \mathbf{S}$ coupling introduces an asymmetry into the scattering cross section.

Let's investigate some of the effects of equation 5.22 through an example. Consider 
(a)

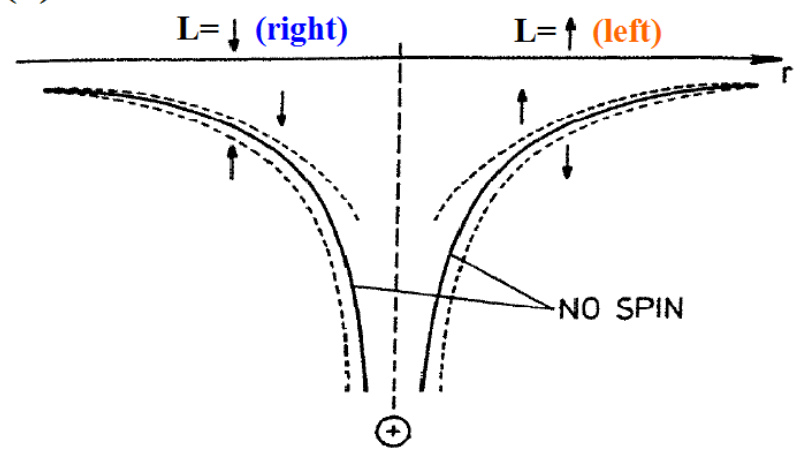

(b)

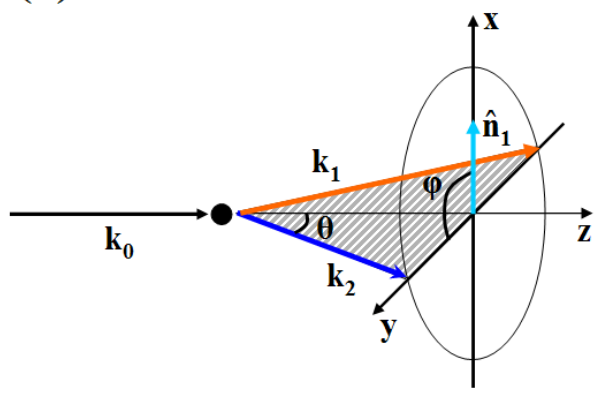

Figure 5.1. (a)Scattering potential of a nucleus: solid line gives the plain Coulomb potential, while including the spin-orbit interaction gives the dotted lines. Figure adapted from ref. 140. (b) Scattering geometry, details in text.

scattering an unpolarized beam from from $\mathbf{k}_{\mathbf{0}}$ to $\mathbf{k}_{\mathbf{1}}$ as shown in Figure 5.1(b). From section 5.1 we know that the unpolarized beam can be modeled as equal numbers of electrons polarized spin-up and -down along any axis, so let's take the incident beam to be made up of equal numbers of electrons with spin parallel and antiparallel to $\hat{x}$. The number of electrons scattered to $\mathbf{k}_{1}$ with spin parallel, $N_{\uparrow}$, will be proportional to the cross section given by equation 5.22 with $\mathbf{P} \cdot \hat{\mathbf{n}}_{\mathbf{1}}=+1$, and the number scattered to $\mathbf{k}_{1}$ with spin antiparallel, $N_{\downarrow}$ will be proportional to the cross section using $\mathbf{P} \cdot \hat{\mathbf{n}}=-1$. Immediately we can see that because the cross section is spin-dependent, the scattered beam will become polarized! The scattered beam at $\mathbf{k}_{1}$ has a polarization given by

$$
\mathbf{P}=\frac{N_{\uparrow}-N_{\downarrow}}{N_{\uparrow}+N_{\downarrow}}=\frac{\sigma_{\uparrow}-\sigma_{\downarrow}}{\sigma_{\uparrow}+\sigma_{\downarrow}}=\frac{[1+S(\theta)]-[1-S(\theta)]}{[1+S(\theta)]+[1-S(\theta)]}
$$

finally resulting in

$$
\mathbf{P}=S(\theta) \hat{\mathbf{n}}_{\mathbf{1}} .
$$

Because $\hat{\mathbf{n}}_{\mathbf{1}}$ is directed along the $x$-axis, the beam will be polarized with magnitude $P=S(\theta)$ oriented 'up' along $x$. Following the same analysis, we find that the beam scatted to $\mathbf{k}_{2}$ becomes oppositely polarized with magnitude $P=S(\theta)$ oriented 'down' along $x$. This 'leftright' asymmetry can be seen to originate in the symmetry of the scattering potential of Figure 5.1.

We can also analyze the total number of electrons scattered to $\mathbf{k}_{1}$ and $\mathbf{k}_{2}$, now for an 
incident beam which may be polarized. We can define the normalized asymmetry in the number scattered left and right by

$$
A_{S O}(\theta)=\frac{N_{L}-N_{R}}{N_{L}+N_{R}}
$$

$N_{L}$ and $N_{R}$ are proportional to the cross sections given by equation 5.22 again. Taking note of the relative direction of $\hat{\mathbf{n}}$ in each case, we have

$$
A_{S O}(\theta)=\frac{\left(1+S(\theta) \mathbf{P} \cdot \hat{\mathbf{n}}_{\mathbf{1}}\right)-\left(1-S(\theta) \mathbf{P} \cdot \hat{\mathbf{n}}_{\mathbf{1}}\right)}{\left(1+S(\theta) \mathbf{P} \cdot \hat{\mathbf{n}}_{\mathbf{1}}\right)+\left(1-S(\theta) \mathbf{P} \cdot \hat{\mathbf{n}}_{\mathbf{1}}\right)}
$$

where $\mathbf{P}$ is the polarization of the incident beam. Therefore, we find that

$$
A_{S O}(\theta)=S(\theta) \mathbf{P} \cdot \hat{\mathbf{n}}_{\mathbf{1}}
$$

Electrons are preferentially scattered one way or the other depending on their spin direction. Again we see a 'left-right' asymmetry, this time in the total number of incident polarized electrons scattered to $\mathbf{k}_{1}$ and $\mathbf{k}_{2}$.

Thus the Sherman function describes both the magnitude of the induced polarization of an incident unpolarized beam scattered to a particular direction and the magnitude of the asymmetry of a totally polarized beam scattered to the 'left' and 'right'. An important aspect to note is that the Sherman function is only 'sensitive' to polarization components perpendicular to the scattering plane $(\hat{\mathbf{n}})$. In other words, the Sherman function only changes the magnitude of the polarization component along the direction perpendicular to the scattering plane (although any component parallel to the scattering plane is rotated about $\hat{\mathbf{n}}$, as described by Kessler ${ }^{140}$ ). The 'left-right' scattering asymmetry induced by the Sherman function is only a function of the incident polarization component perpendicular to the scattering plane as well.

The latter effect is the basis for utilizing Mott scattering to measure spin polarization. This is clear by inverting equation 5.27 to give

$$
P_{n}=\frac{1}{S(\theta)} A_{S O}(\theta)
$$

Thus a measurement of the normalized differential count rate of electrons scattered to opposing angles in the same scattering plane gives the incident polarization component normal 
(a)

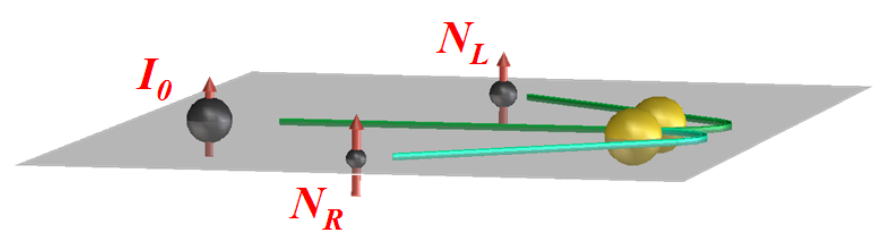

(b)

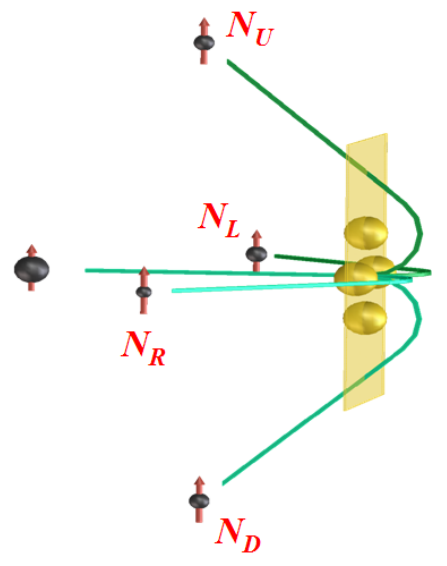

Figure 5.2. Cartoon schematic of the working principles of a Mott polarimeter.

to the scattering plane, assuming the Sherman function is a know quantity. For example, in a Mott polarimeter, an electron beam with intensity $I_{0}$ and of unknown polarization is directed towards atomic scattering potentials (usually Au nuclei) as depicted in Figure 5.2(a). Two possible scattering paths at opposite angles within the same scattering plane are depicted, with opposite orbital motions around the nuclei. Due to the polarization of the incident beam perpendicular to the scattering plane, the scattering rate to the left and right are asymmetric (depicted in the figure as different sized spheres in the scattered beams). Individual electron detectors placed along these paths can then measure the normalized asymmetry defined in equation 5.25. With a known Sherman function, this constitutes a measurement of the perpendicular component of the incident polarization (as depicted in the figure). In the end, use of the spin-orbit interaction allows a measurement of electron polarization to be reduced to a measurement of relative intensity.

Figure 5.2 show that a similar relative intensity measurement can be made between the count rates $N_{U}$ and $N_{D}$ lying in the vertical scattering plane as shown. This scattering plane is only sensitive to polarization components along the direction out of the plane of the figure. In the particular case shown, as the incident beam is polarized completely in the vertical direction, there is no measured asymmetry between $N_{U}$ and $N_{D}$. However, this illustrates the concept that a Mott polarimeter, equipped with two sets of opposing electron detectors along perpendicular scattering planes, can then measure two orthogonal 
components, thus yielding the complete transverse polarization of the incident beam. The Mott polarimeter is insensitive to any longitudinal polarization component. This component can be effectively measured by rotating it into the transverse direction by passing the beam through $90^{\circ}$ electrostatic deflector or a Wien filter prior to entering the polarimeter, as discussed in Chapter 3.6.3 of Kessler. ${ }^{140}$

\subsubsection{Polarimeter efficiency}

As its function is to measure the polarization of electron beams, a polarimeter's efficiency is closely linked to its statistical error in polarization measurement. A Mott polarimeter's error in polarization measurement is given by equation 5.28 as

$$
\Delta P=\frac{1}{S(\theta)} \cdot \Delta A_{S O}
$$

The error in $A_{S O}$ is related to the statistical error in the total counts recorded in the left and right detectors as

$$
\begin{aligned}
(\Delta A)^{2} & =\left(\frac{\partial A}{\partial N_{L}} \cdot \Delta N_{L}\right)^{2}+\left(\frac{\partial A}{\partial N_{R}} \cdot \Delta N_{R}\right)^{2} \\
& =\left(\frac{2 N_{R}}{\left(N_{L}+N_{R}\right)^{2}}\right)^{2}\left(\Delta N_{L}\right)^{2}+\left(\frac{-2 N_{L}}{\left(N_{L}+N_{R}\right)^{2}}\right)^{2}\left(\Delta N_{R}\right)^{2} .
\end{aligned}
$$

Assuming the errors in $N_{L}$ and $N_{R}$ are governed by Poisson statistics, we can substitute $\Delta N_{i} \rightarrow \sqrt{N_{i}}$, as well as substitute $N_{L}+N_{R}=N$ to give

$$
(\Delta A)^{2}=\frac{4\left(N_{R}^{2} N_{L}+N_{L}^{2} N_{R}\right)}{N^{4}}=\frac{4 N_{R} N_{L}}{N^{3}} .
$$

From the definition of $A_{S O}$ and equation 5.28 we can write

$$
P=\frac{1}{S(\theta)} \frac{N_{L}-N_{R}}{N}
$$

from which we can write

$$
1-P^{2} S(\theta)^{2}=\frac{N^{2}-\left(N_{L}-N_{R}\right)^{2}}{N^{2}}=\frac{4 N_{R} N_{L}}{N^{2}}
$$

Combining equations 5.30 and 5.32 gives

$$
(\Delta A)^{2}=\frac{1-P^{2} S(\theta)^{2}}{N}
$$


resulting in

$$
\Delta P=\sqrt{\frac{1}{N}\left(\frac{1}{S(\theta)^{2}}-P^{2}\right)} .
$$

Unfortunately, the Sherman function in all current polarimeters are fairly small such that $1 / S(\theta)^{2}>P^{2}$, so this can be further simplified to just

$$
\Delta P=\sqrt{\frac{1}{N S(\theta)^{2}}} .
$$

Thus we see that the error in a polarization measurement goes down with increased total recorded counts at a rate scaled by the Sherman function squared. In discussing polarimeter efficiency, it is important to note that $N$ in equation 5.35 is the total number of recorded electrons scattered to the detector - not the total number of electrons sent into the polarimeter. It is then more insightful to express the error in polarization measurement as a function of the number of electrons sent into the polarimeter, $N_{0}$, as

$$
\Delta P=\sqrt{\frac{1}{N_{0}\left(\frac{N}{N_{0}}\right) S(\theta)^{2}}}=\frac{1}{\sqrt{N_{0} \times(\mathrm{FOM})}}
$$

where we have defined a 'figure of merit' (FOM) for a Mott-type polarimeter as

$$
\mathrm{FOM}=\frac{N}{N_{0}} S_{\text {eff }}^{2}=\frac{I}{I_{0}} S_{\text {eff }}^{2},
$$

expressed in terms of either the ratio of total counts to number of input electrons, or the ratio of count rate to input current. The relative error in measurement of simply the intensity of a beam of electrons, $\Delta N_{0} / N_{0}$, scales as $1 / \sqrt{N_{0}}$. From equation 5.36 we see that the corresponding relative error of a polarization measurement, $\Delta P / P$ scales as $1 / \sqrt{P^{2} N_{0}(\mathrm{FOM})}$. With $|P|<1$, this means measuring a beam's polarization takes more than FOM times longer than just its spin-integrated intensity. This explains the term 'figure of merit' as it is an effective quantitative measure of a polarimeter's efficiency. Current polarimeters have FOMs of only around $10^{-4}$, explaining why electron polarization experiments are so difficult. In defining the FOM in equation 5.37, we have used $S_{\text {eff }}$ in place of $S(\theta)$. As the electron detectors in actual polarimeters typically subtend a finite solid angle including a range of $\Delta \theta$, the term 'effective Sherman function' is used to distinguish from the pure $S(\theta)$ which applies to an exact $\theta$. 
The goal of spin polarimeter design is to try to maximize both $S_{\text {eff }}$ and $N / N_{0}$; unfortunately, it is usually the case that increasing one term will decrease the other. Simply maximizing the FOM is not always the best route, either. For instance, the FOM in some cases may be increased overall at the expense of $S_{\text {eff }}$. However, most polarimeters will have an instrumental asymmetry between $N_{L}$ and $N_{R}$ that is not related to polarization. If $S_{\text {eff }}$ is too small, then systematic experimental errors such as the instrumental asymmetry will dominate the measurement error instead of the statistical error. ${ }^{140}$ Therefore, it is prudent to use the FOM when considering polarimeter efficiency with the understanding that too small a $S_{\text {eff }}$ (or a too large instrumental asymmetry) will be unacceptable, especially if measuring small polarizations is desirable.

\subsubsection{Mott polarimeter design and usage}

For Mott scattering, equation 5.37 impacts polarimeter design in a number of ways. Spin-orbit coupling is strongly dependent on the nuclear charge (scales as $Z^{4}$, see section 1.2.3 equation 1.29), and so scattering from high-Z nuclei will give larger Sherman functions. Because of this, Mott detectors normally scatter from $\mathrm{Au}(\mathrm{Z}=79)$ foils. $\mathrm{Au}$ is also preferred over other large- $Z$ metals because it is fairly non-reactive and does not form thick oxide layers. A Au surface, even in poor vacuum, will give very stable Mott scattering measurements without degradation over very long time periods. Also, Au can be made into extremely thin foils. One problem for any type of scattering instrument is the possibility of having multiple scatterings in the target before reaching the detector. An electron arriving at a detector after multiple scatterings will degrade the effective Sherman function, and so thin foils are preferred to decrease the chance of plural scatterings.

Two variables which can have a large effect on performance is the scattering angle, $\theta$, and the incident kinetic energy. The Sherman function can be a strong function of the scattering angle. From equations 5.23 and 5.24 we see that the Sherman function can be expressed as

$$
S(\theta)=\frac{\sigma_{\uparrow}-\sigma_{\downarrow}}{\sigma_{\uparrow}+\sigma_{\downarrow}} .
$$

Even with a large $\mathrm{Z}$ nucleus like $\mathrm{Au}$, the spin-orbit interaction is still quite small, suggesting 
only small values of $\sigma_{\uparrow}-\sigma_{\downarrow}$. Thus, the highest values of $S(\theta)$ are typically found only where $\sigma_{\uparrow}+\sigma_{\downarrow}$ is quite low. In other words, the Sherman function is usually maximum where the total cross section is near a minimum, forcing a compromise between $S(\theta)$ and $I / I_{0}$. As it turns out, the Sherman function only becomes sizable at fairly large backscattering angles, usually around $\theta \sim 120^{\circ}$. Intuitively, these large scattering angles give low total cross sections and ensure a very low value for $I / I_{0}$.

The kinetic energy of the scattering also becomes a difficult compromise. Maximum Sherman functions from $\mathrm{Au}$ foils generally increase with kinetic energies well over $100 \mathrm{keV}$ to even $1 \mathrm{MeV} .{ }^{143}$ Of course, at these energies, the backscattering cross sections become extremely low, and the instrumental difficulties of these high voltages become far too cumbersome. Mott scattering at low energy $(\sim 300 \mathrm{eV})$, however, can be effective when scattering from $\mathrm{Hg}$ vapor due to diffractive effects creating cross section minima where the Sherman function can become sizable. ${ }^{140}$ Still, the FOM suffers from very low total cross sections, partly due to the low density of nuclei. Plural scattering in solid targets becomes too significant at these energies, however, and thus cannot be used.

Although some designs feature slight FOM improvements here and there, the above issues well illustrate that it is difficult to achieve a high FOM due to the inverse relationships between $S_{\text {eff }}$ and $I / I_{0}$. A particularly good review of these and many other details of Mott polarimetry is one by Gay and Dunning. ${ }^{144}$ When the dust settles, a well designed high energy $(100 \mathrm{keV})$ Mott polarimeter can operate with a FOM $\sim 1 \times 10^{-4}$. More recently, lower energy Mott polarimeters have been developed which can operate at closer to 20 $\mathrm{keV}$ while maintaining a fairly high $S_{\text {eff }}$ by filtering out inelastically scattered electrons through a retarding potential. These retarding potential Mott polarimeters, or 'mini-Motts', allow a much smaller physical size than the high energy type, and have become the most commonly used in photoemission work. A popular design with high efficiency developed at Rice University, ${ }^{145}$ representing the current state-of-the-art, is illustrated in Figure 5.3(a). It operates at $25 \mathrm{keV}$ and $\theta=120^{\circ}$ scattering, and achieves effective Sherman functions between -0.15 and -0.25 and $I / I_{0}$ values in the range $2-6 \times 10^{-3}$. These parameters result 
(a)

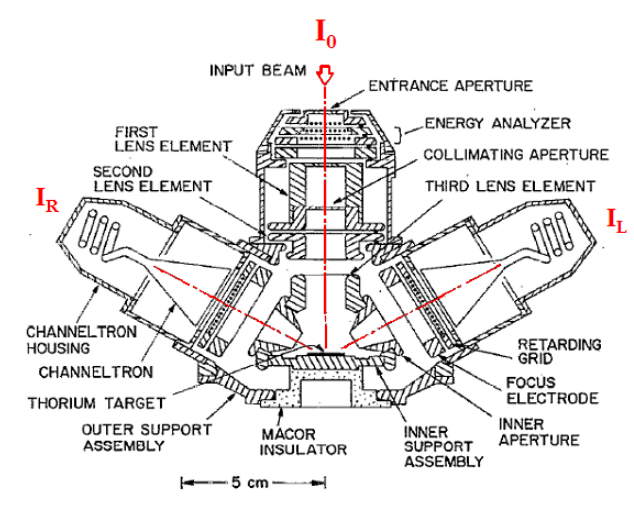

(b)

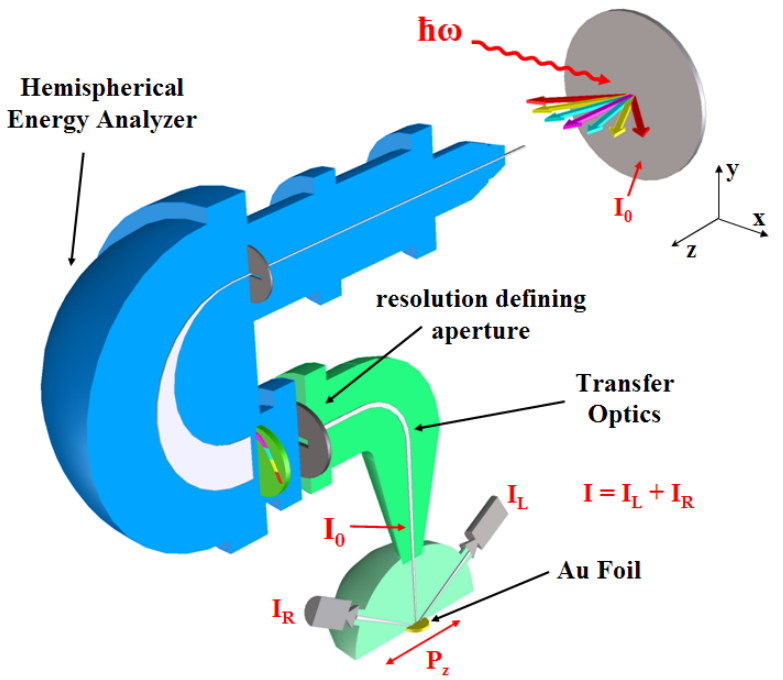

Figure 5.3. (a)Schematic of a retarding potential 'mini-Mott' polarimeter. Figure taken from ref. 145. (b) Diagram of a spin-resolved photoemission setup, coupling a Mott polarimeter to a hemispherical analyzer. See text for details.

in a total efficiency, or FOM of $\sim 1.6 \times 10^{-4}$. A version developed as recently as $2007^{146}$ provides the same parameters, although with a further reduction in overall size.

These state-of-the-art numbers readily demonstrate how difficult spin resolved experiments are. The FOM of $10^{-4}$ is the source of the detector efficiencies used in section 3.4. In particular, one should note that a $I / I_{0}$ value of $2 \times 10^{-3}$ means that for every 1000 electrons sent into the polarimeter, only 2 are actually counted! Many current spin-resolved ARPES systems ${ }^{105,106,107,108}$ are built by coupling a 'mini-Mott' polarimeter to a hemispherical analyzer (SDA) as shown in Figure 5.3(b). It is clear that the polarimeter effectively forms a single spatial channel detector - when coupled to an SDA, only a single energy/angle channel can be measured at a time, as described in section 3.4. The $N_{L}$ and $N_{R}$ pair of electron detectors shown in the figure are sensitive to the polarization component along the sample $z$-axis. Many Mott polarimeters have a second pair of detectors oriented at a $90^{\circ}$ rotation around the Au foil normal, giving a simultaneous measurement of the polarization component along the $x$ axis. The TOF-based spin-resolved photoemission system mentioned in section 4.3 also use a similar mini-Mott as the spin-polarimeter. ${ }^{116,147}$

These Mott polarimeters are popular for spin-resolved photoemission experiments be- 
cause they are well understood and well established and give a steady Sherman function due to the stable target quality. One drawback is the requirement of a high quality design and precise alignment to reduce instrumental asymmetries, well discussed in ref. 144. An important aspect to any polarimeter is calibration: the effective Sherman function, $S_{\text {eff }}$, must be known for an accurate measurement of $P$ through equation 5.28. An attractive quality of Mott polarimeters is the ability to calibrate $S_{\text {eff }}$ with measurements at different $\mathrm{Au}$ foil thicknesses to extrapolate to 'zero thickness' where the Sherman function can be reliably based on single atom calculations. ${ }^{144}$ Once this is performed, $S_{\text {eff }}$ is known for the complete series of foil thicknesses, and this can be approximately a 'one-time' calibration due to the long life time of the Au foils in even poor vacuum. One of the largest reasons for the popularity of Mott-type detectors is that they are also capable of $10^{-4}$ FOMs, similar to the highest of any regularly used spin polarimeter. This FOM is still quite low compared to spin-integrated measurements, and so many other schemes have been investigated and developed.

\subsubsection{Other techniques}

Due to the importance of spin-resolved experiments, it is not surprising that other polarimetry techniques have been developed. A particularly interesting example is the LEED polarimeter. ${ }^{140,95,16}$ In Mott polarimeters, the Au targets are polycrystalline, and the scattering was treated as from single atoms. With single crystal targets, the coherent scattering of low energy electrons from a periodic array of atoms gives strong diffraction effects with intensity distributions very different from that of foils. This of course is the basis of Low Energy Electron Diffraction (LEED), where intense Bragg reflection spots are related to the target crystal structure. At first approximation, equivalent Bragg reflections should give equal intensities. However, for large Z crystals, the same spin-orbit induced 'leftright' intensity asymmetry found in Mott scattering is found in LEED spots with polarized incident electrons. The large scattering angles in LEED suggest large asymmetries (Sherman functions) may be present. The intense diffraction reflections suggest the possibility of finding simultaneous high reflectivities, or $I / I_{0}$, along with a high $S_{\text {eff }}$. 
Just as Mott scattering can induce spin polarization from an unpolarized beam, LEED spots from high-Z crystals can become spin-polarized with an unpolarized incident beam. This was first shown for W crystals by O'Neill et. al. ${ }^{148}$ in 1975 . The left-right intensity asymmetry in equivalent LEED spots across a mirror plane due to the polarization of the incident beam was first shown by Kirschner and Feder ${ }^{149}$ in 1979, and they suggested to use this asymmetry as the basis for a spin polarimeter. In such a polarimeter, the intensity of opposing 'equivalent' LEED spots (e.g. the $(1,0)$ and $(\overline{1}, 0)$ spots) take on the same role as $N_{L}$ and $N_{R}$ in regular Mott polarimetry. Subsequent polarimeters based on measuring the asymmetry of LEED spot intensity from $\mathrm{W}$ crystals have indeed been developed. ${ }^{95,16}$ The error in the polarization measurement in the LEED method is given by the same statistics as in Mott scattering, and so the same FOM can be used to quantify efficiency (section 5.2.2). It was found that the FOM is maximized in the $(2,0)$ vs. $(\overline{2}, 0)$ LEED spots of a W(100) surface. The W(100) surface is ideal because it has a large Z, it has a straightforward surface cleaning procedure, and the (100) surface has 4-fold symmetry and is unreconstructed at room temperature. The 4-fold symmetry is useful as the orthogonal pairs of opposite LEED spots, $(2,0)$ vs. $(\overline{2}, 0)$ and $(0,2)$ vs. $(0, \overline{2})$, can simultaneously measure the incident polarization components along 2 orthogonal directions, similar to the Mott polarimeter designs with two pairs of left-right electron detectors at $90^{\circ}$.

The $(2,0)$ LEED spots result in a $S_{\text {eff }}$ of -0.27 and a $I / I_{0}$ of $2.2 \times 10^{-3}$ for a total FOM of $1.6 \times 10^{-4} .{ }^{95}$ Although this is similar to state-of-the-art Mott polarimeters, the operating scattering energy is only $104.5 \mathrm{eV}$ rather than $20-100 \mathrm{keV}$. These low energies avoid the instrumental difficulties associated with high voltage, while Mott scattering at such low energies produces orders of magnitude lower FOMs. ${ }^{144}$ Without high voltage, LEED polarimeters can be designed in simple and small instruments. An attractive characteristic of the LEED polarimeter is the possibility of calibrating the $S_{\text {eff }}$ through double scattering experiments with two $\mathrm{W}$ crystals. ${ }^{95}$ Additionally, polarimeter parameters are fairly insensitive to angle and energy of incidence, allowing energy widths of up to $3 \mathrm{eV}$ to be analyzed without a significant drop in $S_{\text {eff }}$, as well as allowing a couple degrees of beam misalignment without introducing large instrumental asymmetries. ${ }^{16}$ As LEED is intrinsically 


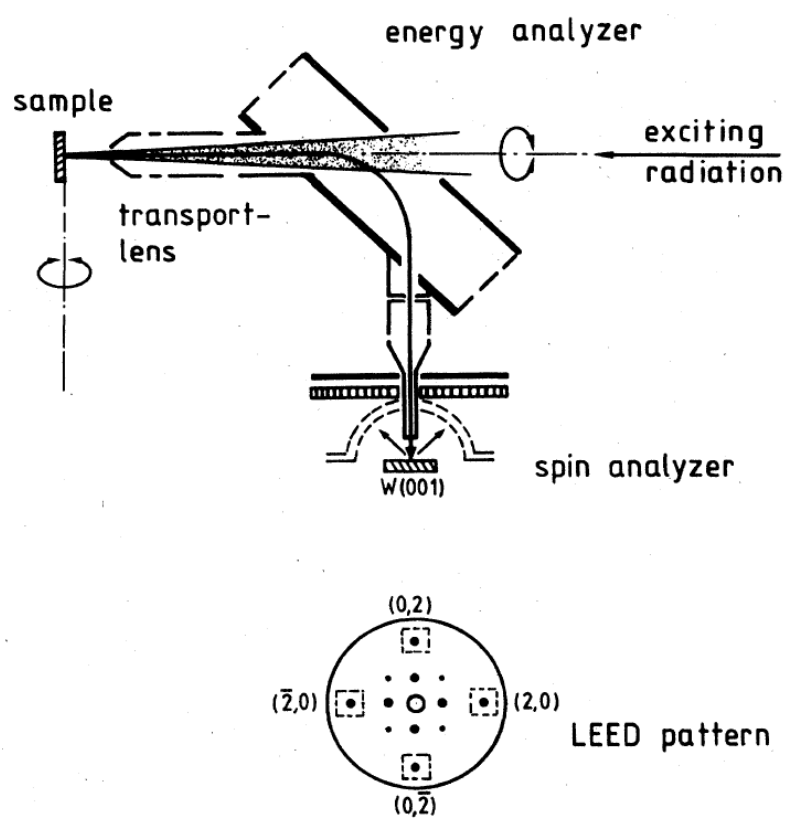

Figure 5.4. Schematic of a spin- and momentum-resolved spectrometer with a LEED polarimeter. Below: schematic of the observed LEED pattern; squares indicated beams used for polarization analysis. Figure taken from ref. 150.

highly surface sensitive, its analyzing power requires a clean surface and UHV conditions for prolonged use, making it a suitable technique only for experiments already requiring UHV. One reason $\mathrm{W}$ is ideal, is it can be refreshed with only periodic high temperature flashing. The coupling of such a LEED polarimeter to an electrostatic cylindrical mirror analyzer for spin, momentum, and energy resolved measurements was achieved by Oepen et. al. ${ }^{150}$ and is shown in Figure 5.4. The use of a LEED polarimeter has also been utilized by the Aeschlimann group ${ }^{151}$ for a variety of spin-resolved photoemission experiments (e.g. refs. $152,153,154,80,155)$. Although an exciting technique, the $10^{-4}$ FOM still represents a very large drop in efficiency.

Additional alternative spin-orbit based schemes have been developed such as the adsorbed current polarimeter ${ }^{95,16}$ and the low energy diffuse scattering polarimeter, ${ }^{156,144,157}$ but all seem to provide similar or less promising characteristics and FOMs. A fantastic review directly comparing the practical usage of an assortment of these spin-orbit based polarimeters is found in ref. 158. In general spin-orbit based polarimeters can provide reliable spin polarization measurements, but with low efficiencies which severely limits the 
practical resolutions that experiments such as ARPES can be performed with. Additional development of spin polarimetry techniques are thus highly desirable.

\subsection{Exchange polarimetry}

\subsubsection{Exchange scattering}

Section 1.2.5 introduced another spin-dependent interaction: exchange coupling. That the exchange interaction can produce spin-dependent electron-electron scattering effects can be seen as follows. Consider the scattering of two electrons, for example as in Figure 1.4. From standard scattering theory, the resulting spatial wavefunction should have the asymptotic form

$$
\Psi=e^{i \mathbf{k} \cdot \mathbf{r}}+f(\theta) \frac{e^{i \mathbf{k} \cdot \mathbf{r}}}{r}
$$

where $\mathbf{k}=\mathbf{k}_{\mathbf{1}}-\mathbf{k}_{\mathbf{2}}$ and $\mathbf{r}=\mathbf{R}_{\mathbf{1}}-\mathbf{R}_{\mathbf{2}}$, in terms of the wavevectors and positions of the two electrons. Because they are identical particles, the two events in Figure 1.4 cannot be distinguished. The required symmetrization of the total wavefunction (see section 1.2.4) results in a spatial wavefunction of

$$
\Psi=e^{i \mathbf{k} \cdot \mathbf{r}} \pm e^{-i \mathbf{k} \cdot \mathbf{r}}+[f(\theta) \pm f(-\theta)] \frac{e^{i \mathbf{k} \cdot \mathbf{r}}}{r}
$$

where the $+(-)$ is for the singlet (triplet) spin state. This results in a cross section for scattering by angle $\theta$ of

$$
\begin{aligned}
\sigma(\theta) & =|f(\theta) \pm f(\pi-\theta)|^{2} \\
& =|f(\theta)|^{2}+|f(\pi-\theta)|^{2} \pm 2 \operatorname{Re}\left[f^{*}(\theta) f(\pi-\theta)\right]
\end{aligned}
$$

The third term on the right shows that there is a difference in cross section depending on the spin state (singlet or triplet) of the scattering electrons!

In the case of Mott (electron-nucleus) scattering in section 5.2.1, spin dependence is described through the addition of a spin-orbit potential, $V_{L S} \propto \mathbf{L} \cdot \mathbf{S}$, resulting in the total potential in Figure 5.1(a). In the case of exchange (electron-electron) scattering, spin dependence is similarly introduced through the addition of an exchange potential, 
(a)

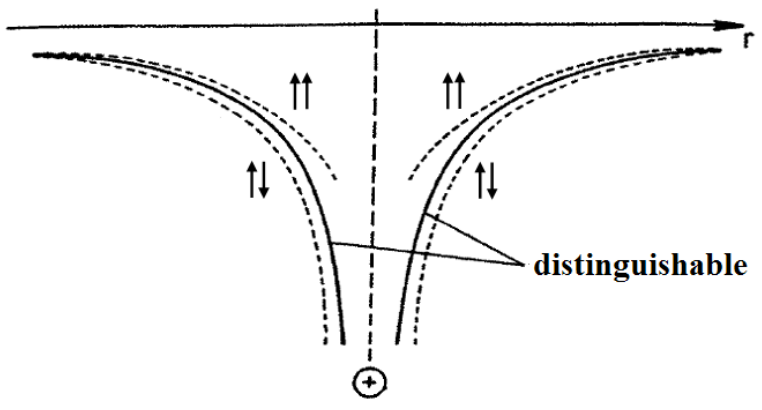

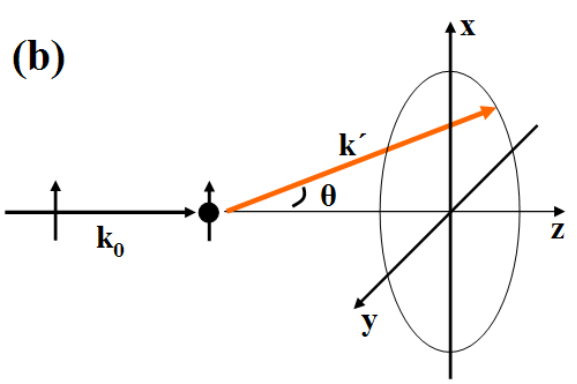

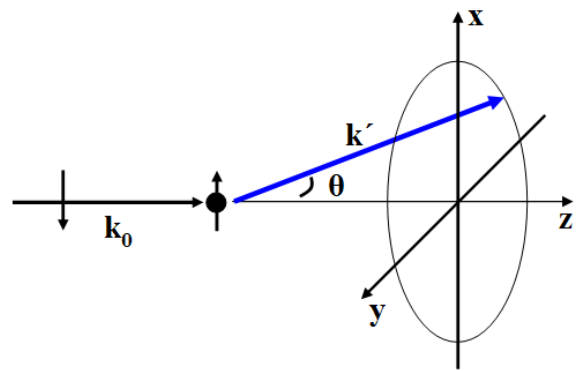

Figure 5.5. Exchange scattering analogue to Figure 5.1. (a) Potential of electron-electron scattering: solid line gives the plain Coulomb potential, assuming the electrons are distinguishable particles. Including the exchange potential gives the dotted lines for spin singlet $(\uparrow \downarrow)$ and triplet $(\uparrow \uparrow)$ alignments. (b) Scattering geometry, details in text.

$V_{\text {ex }}$. Though both potentials bring about spin dependent scattering, there are important differences. For instance, there is a clear 'left-right' asymmetry (see Figure 5.1(a)) due to the effective spatial dependence of the $\mathbf{L} \cdot \mathbf{S}$ term in spin-orbit scattering. There is no equivalent left-right asymmetry in exchange scattering; in other words, the analogous plot of the scattering potential with exchange is symmetric about $\mathrm{r}=0$, as shown in Figure 5.5(a). From equation 1.61, we see that the exchange potential can be expressed as $V_{\text {ex }} \propto \mathbf{S}_{1} \cdot \mathbf{S}_{2}$. This clearly illustrates that in contrast to the spin-orbit case, specific spin orientation with respect to the direction of propagation or the scattering plane is irrelevant; only the relative spin alignment between the two scattering electrons is important. As mentioned in section 1.2.5, this is due to the Hamiltonian having no explicit spin dependence, which enters only through the wavefunction symmetry. Instead of the 'left-right' asymmetry in spin-orbit scattering (e.g. between $\mathbf{k}_{1}$ and $\mathbf{k}_{2}$ in Figure 5.1), there is only an 'up-down' asymmetry in exchange scattering. For instance there is an asymmetry in the cross section for scattering from $\mathbf{k}_{0}$ to $\mathbf{k}^{\prime}$ depending on whether the two electron spins are (at least partly) parallel or antiparallel, as diagrammed in the upper and lower panels of Figure 5.5(b), respectively. 
Such exchange based scattering effects have been utilized in high energy $(1 \mathrm{keV}-1 \mathrm{GeV})$ polarimetry techniques referred to as Møller scattering. ${ }^{140}$ In a Møller scattering experiment, energetic electron beams are directed through a magnetized Fe foil (which has an imbalance of spin-up and -down electrons along the magnetization direction). The asymmetry in measured count rates at certain angles for opposing foil magnetization directions is thus related to the beam polarization component along the magnetization directions. As the film can be magnetized parallel/antiparallel to the beam direction, Møller scattering can be sensitive to longitudinal beam polarization, in contrast to Mott scattering. Because of this, Møller scattering has traditionally been used for high energy $\beta$-decay experiments. The equivalent of the Sherman function, however, is quite low $(<8 \%)$ and so it is not used for other experiments. A more complete discussion can be found in ref. 140.

Exchange effects can be utilized in other ways for more applicable polarimetry techniques. For instance, the mean free paths of electrons in ferromagnetic solids tends to be spin dependent, with the exchange interaction the root cause. This results in spin dependent transmission through magnetic thin films, which could then be used as a 'spin-filter'. This was suggested by Schönhense and Siegmann ${ }^{159}$ who estimated that the equivalent FOM of such a filter made of an Fe film $1.0 \mathrm{~nm}$ thick would be as high as $7 \times 10^{-2}$ ! Practically, however, it is difficult to physically construct such a thin free-standing film. As the film thickness increases, the average transmission decreases exponentially, drastically reducing the equivalent of the $I / I_{0}$ term in the Mott FOM. Nevertheless, the performance of a spinfilter made by a $1 \mathrm{~nm}$ Co film sandwiched between a $21 \mathrm{~nm}$ and $2 \mathrm{~nm}$ Au layers has been investigated. ${ }^{160,161}$ With surface cesiation, the total transmission was $3 \times 10^{-4}$ with an effective Sherman function of $S_{\text {eff }}=0.4$ for a total FOM of $\sim 5 \times 10^{-5}$. Of course if it were possible to make the free standing films thinner, the increased transmission would lead to a much improved FOM.

In addition to transmission, exchange also effects electron reflection from magnetic surfaces. In the LEED-based spin-orbit polarimeters discussed in section 5.2.4, it was found that diffraction from single crystals gave the possibility of finding overlapping high spinasymmetry $\left(S_{\text {eff }}\right)$ and high reflectivity $\left(I / I_{0}\right)$. The same is found to be true of low en- 

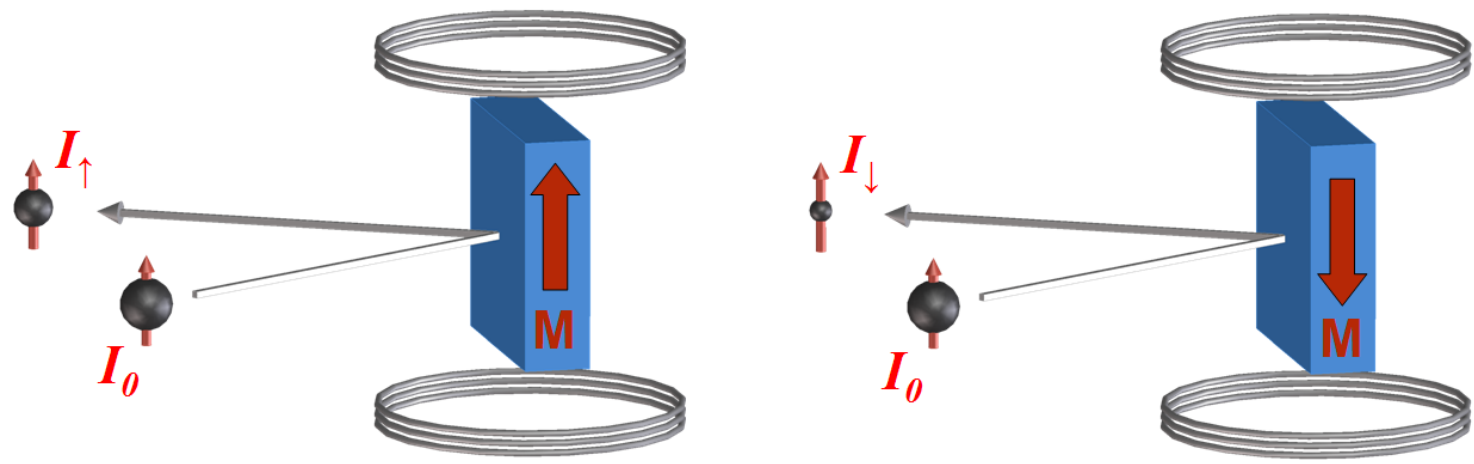

Figure 5.6. Cartoon schematic of working principle of an exchange scattering polarimeter. The differing size of the spheres representing electrons represent differing intensities. Target magnetization is performed by the coils.

ergy scattering from ferromagnetic single crystals, where instead of the spin-orbit interaction it is the exchange interaction supplying the spin asymmetry. Indeed, early works found that the absorbed current and reflected current of electron beams from magnetized $\mathrm{Fe}(001)^{162,163,164,165}$ and $\mathrm{Fe}(110)^{166,167}$ were strongly dependent on whether the incident beam was spin polarized parallel or antiparallel to the crystal magnetization. This asymmetry, along with relatively high average reflectivities $\left(I / I_{0}\right)$, was suggested for use as spin polarimeters, and indeed found immediate such use. ${ }^{164,165}$

It should be stressed again that the spin-orbit variety of diffraction polarimeters utilizes the left-right asymmetry between opposing LEED spots. The exchange based diffraction asymmetry is in the intensity of a single 'LEED spot' between opposite crystal magnetizations (earlier referred to as an 'up-down' asymmetry), meaning that measuring only a single diffracted beam is necessary. This is important as it means the $(0,0)$ diffraction (i.e. specular reflection), can be used, in contrast to spin-orbit based schemes. This is beneficial as specular reflection can have higher intensity than higher order diffractions. Indeed, if extremely low incident kinetic energy electrons are used, the Ewald sphere becomes so small that there are no higher order diffracted beams, leading to increased intensity of the $(0,0)$ specular reflection. ${ }^{168}$ This suggests that a polarimeter based on low energy exchange scattering may give very high efficiencies.

Let's first better define how such a polarimeter would function, as depicted in Figure 5.6. 
An incident beam of electrons of unknown polarization with intensity $I_{0}$ is incident on the scattering target, magnetized in one direction $(\uparrow)$, as on the left of the figure. The rate of electrons specularly reflected in this case is measured as $I_{\uparrow}$. The target is then re-magnetized in the opposite direction $(\downarrow)$, as on the right of the figure, and the rate of electrons specularly reflected is measured as $I_{\downarrow}$. In analogy with equations 5.22-5.28 for Mott polarimetry, the scattered intensities will be proportional to the cross sections which can be expressed as

$$
\sigma(\alpha)=\sigma_{0}(\alpha)\left(1+S^{(e x)}(\alpha) \mathbf{P} \cdot \widehat{\mathbf{M}}\right)
$$

where $\alpha$ is the angle of incidence, measured from the surface normal, $\mathbf{P}$ is the incident polarization, and $\widehat{\mathbf{M}}$ is the scattering target's magnetization direction. The term $\sigma_{0}(\alpha)$ is the cross section in the absence of exchange coupling, and is independent of incident spin or surface magnetization. One can define the measured normalized asymmetry between the two intensities as

$$
A_{e x}=\frac{I_{\uparrow}-I_{\downarrow}}{I_{\uparrow}+I_{\downarrow}}
$$

Use of equation 5.42 shows that the asymmetry is proportional to the incident beam polarization by

$$
A_{e x}=S_{\mathrm{eff}}^{(e x)} \mathbf{P} \cdot \widehat{\mathbf{M}}
$$

At the beginning of the section it was noted that the additional 'exchange potential' could be expressed $V_{e x}=\mathbf{S}_{1} \cdot \mathbf{S}_{2}$. Relating $\mathrm{S}_{1}$ with $\mathbf{P}$ of the incident beam and $\mathbf{S}_{2}$ with $\widehat{\mathbf{M}}$ of the target, we can see that the $\mathbf{P} \cdot \widehat{\mathbf{M}}$ term in equation 5.44 is not surprising. The term $S_{\text {eff }}^{(e x)}$ describes the analyzing power of the scattering process and is thus analogous to the Sherman function in Mott scattering. Due to the parallels, we will also refer to it as the effective Sherman function for exchange polarimeters. If the effective Sherman function is known, then a measurement of the asymmetry in the reflectivity yields the polarization through

$$
P_{m}=\frac{1}{S_{\mathrm{eff}}^{(e x)}} A_{e x} .
$$

Equations 5.44 and 5.45 show that the exchange scattering asymmetry, and thus an exchange polarimeter, is sensitive to the polarization component along the target magnetization axis. 


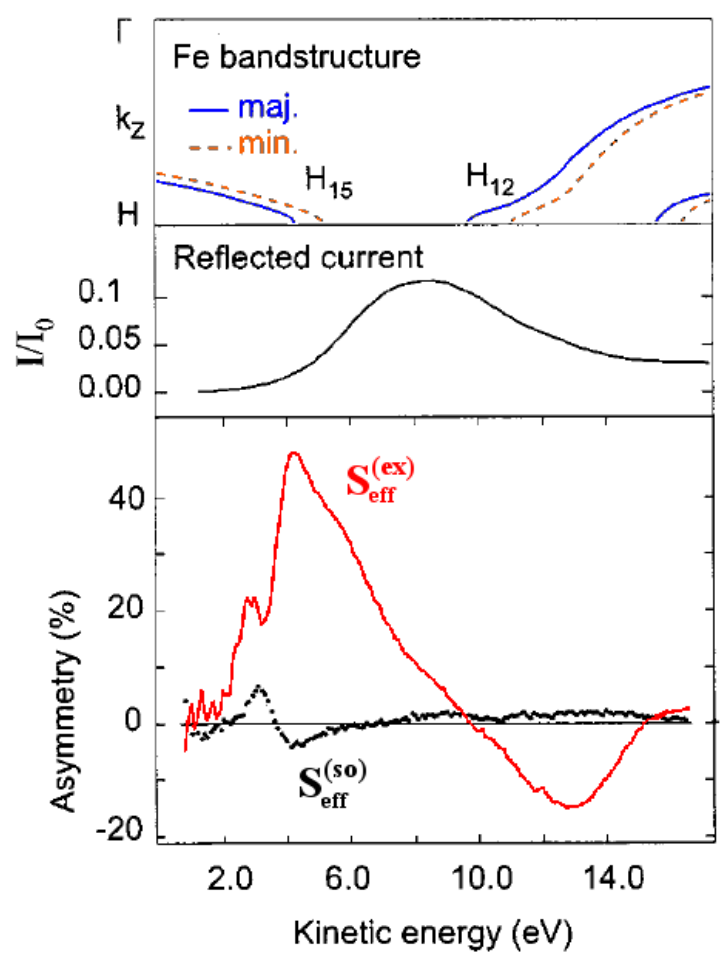

Figure 5.7. Study of the spin asymmetry of reflected intensity from a single crystal Fe(001)$\mathrm{p}(1 \mathrm{x} 1) \mathrm{O}$ target, adapted from ref. 169. (a) Calculated unoccupied bandstructure along the $k_{z}$ direction. (b) Spin integrated reflected intensity. (c) Resulting exchange asymmetry in red. Spin-orbit asymmetry in black.

\subsubsection{Qualitative understanding of exchange asymmetry}

The source of the asymmetry in scattering from magnetic crystals is not really as simple as suggested by the free electron scattering in equation 5.41. Multiple scatterings, inelastic processes, crossing the surface potential barrier, and details of the crystalline bandstructure all add to the complexity of the situation. The exact details of the scattering process is not completely necessary for the successful operation of such a polarimeter however, and the analysis can be treated somewhat empirically. In other words, if the spin dependence in scattering intensity from a given surface can be experimentally measured/calibrated, then it can be used as a polarimeter without complete knowledge of all the complexities. It is, however, interesting to obtain additional qualitative insights into the origin of the asymmetry, perhaps leading to predictions for more ideal surfaces to use. 
Figure 5.7 shows results of Bertacco et. al. ${ }^{169}$ who studied the spin dependent reflectivity of an $\mathrm{Fe}(001)$ crystal with a $\mathrm{p}(1 \mathrm{x} 1)$ oxide overlayer. It was found that the reflectivity, including its spin dependence, could be qualitatively interpreted as due to the unoccupied bandstructure of the target, shown along the $k_{z}$ direction in panel (a). There is a $\sim 5 \mathrm{eV}$ gap centered at about $7 \mathrm{eV}$ above the vacuum level. It is argued that the incident electrons can only penetrate the crystal by coupling to available states in the bandstructure; at energies in the bandgap, the electrons do not enter and are strongly reflected. This argument is reinforced by the average reflectivity shown below in panel(b) where there is a strong, broad maximum centered within the energies of the gap.

The reflectivity asymmetry between incident beams of opposite polarization along the

target magnetization direction is shown in panel (c). The asymmetry $S_{\text {eff }}^{(e x)}$ shows a strong maximum and minimum near each band edge, and can be qualitatively explained as follows. As $\mathrm{Fe}$ is ferromagnetic, its bandstructure in (a) shows pronounced exchange splitting and a spin-dependent gap. For instance, the low energy onset of the gap occurs at lower energy for majority spin electrons than minority electrons. In the energy range where there is a gap for the majority spin, but not a gap yet for the minority spin, it is reasonable to expect that electrons of majority spin will be more strongly reflected than electrons of minority spin. The opposite would then be true at the high energy end of the gap where minority spin will be more strongly reflected. This is exactly what is found in the $S_{\text {eff }}^{(e x)}$ curve in panel(c). While this does not suffice as a complete quantitative explanation, it is reassuring to have a qualitative picture. It also gives reason to expect that targets with large exchange splitting would be ideal candidates for use as a spin polarimeter.

The exact definitions of $S_{\text {eff }}^{(e x)}$ and $S_{\text {eff }}^{(s o)}$ (also shown in panel (c)) are described in the following section.

\subsubsection{More details: spin-orbit contributions}

Ideally, equations 5.42 - 5.45 can accurately describe the scattering from magnetic surfaces. However a more accurate description must also include spin-orbit effects. For 3d 
(a)

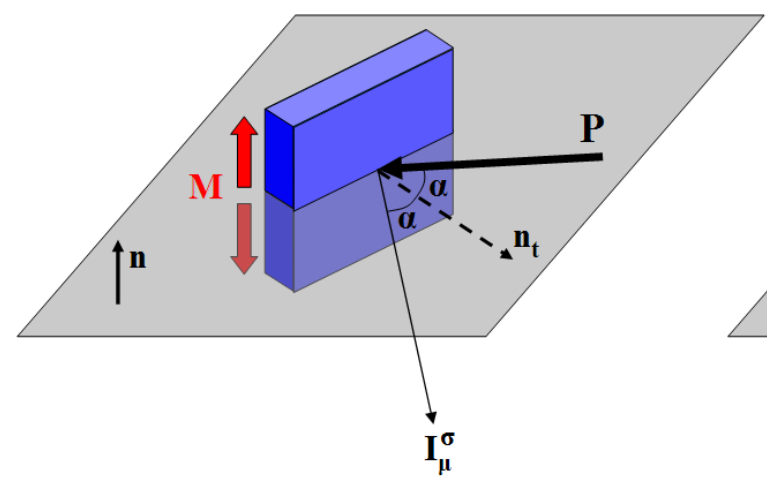

(b)

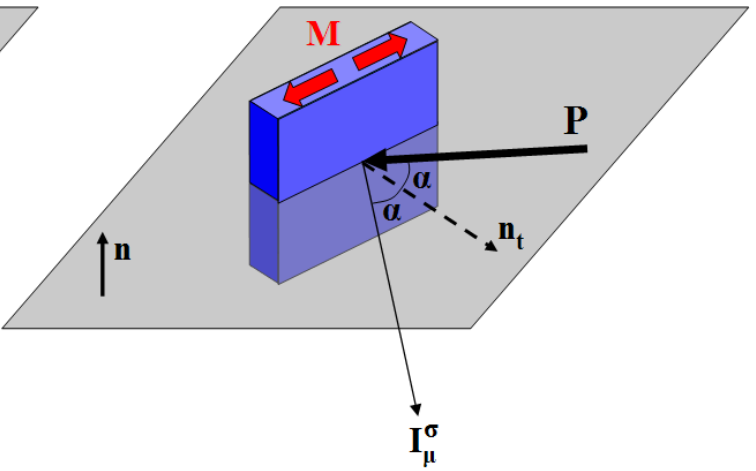

Figure 5.8. Particularly useful scattering geometries: (a) 'Perpendicular' and (b) 'Planar'.

ferromagnetic targets ( $\mathrm{Fe}, \mathrm{Co}, \mathrm{Ni})$, spin-orbit effects are small due to the low-Z nuclei, but they are still of the same order of magnitude as exchange effects, and should not be disregarded. The two spin dependent effects are difficult to separate in general, but they can be approximately separated in two particular scattering geometries (see refs. 140,16): the 'Perpendicular' and 'Planar' geometries shown in Figure 5.8. In both cases, the target crystal is azimuthally oriented so that a mirror plane coincides with the scattering plane. Note that only specular reflection is considered, i.e. the incident beam and outgoing beam both make an angle $\alpha$ with respect to the target normal, $\mathbf{n}_{t}$. From the previous section, it is obvious that there will be exchange effects if the incident beam has a polarization component parallel to the target magnetization axis in either case. There will be an exchange induced 'up-down' $(\mathbf{P} \cdot \widehat{\mathbf{M}})$ asymmetry in the reflected intensity when the magnetization direction is flipped (incident beam kept constant). If the beam has a polarization component along the scattering plane normal, $\mathbf{n}$, it should also be clear that there can be spin-orbit effects as well, as the reflected beam is scattered 'left'. From section 5.2.1 we know that the spin-orbit 'left-right' $(\mathbf{P} \cdot \hat{\mathbf{n}})$ asymmetry will manifest itself here as an asymmetry in the reflected intensity when the incident beam polarization is flipped (target magnetization kept constant).

Let's first consider the 'Perpendicular' geometry in Figure 5.8(a). In this case, both exchange and spin-orbit effects are only sensitive to the incident polarization component 
along the scattering plane normal, and so we will only consider the polarization along $\mathbf{n}$. Assuming the polarization (although the magnitude is unknown) can be flipped, there are four reflected intensities which can be measured, corresponding to the four combinations of polarizations and magnetizations along the $\mathbf{n}$ axis. These intensities will be labeled as $I_{\mu}^{\sigma}$, where $\mu$ is ' $+1^{\prime}\left({ }^{6}-1^{\prime}\right)$ when the magnetization is antiparallel (parallel) to $\mathbf{n}$ (this orientation of signs is due to the electron magnetic moment being opposite its spin direction), and $\sigma$ is ' +1 ' ('-1') when the incident polarization is parallel (antiparallel) to $\mathbf{n}$. Both exchange and spin-orbit effects can be approximately accounted for by essentially combining equations 5.22 and 5.42 into a single expression (here in terms of intensity rather than cross section) as

$$
I_{\mu}^{\sigma}=I(\theta)\left(1+\mu \sigma P S_{\mathrm{eff}}^{(\mathrm{ex})}+\sigma P S_{\mathrm{eff}}^{(\mathrm{so})}\right)
$$

where $S_{\text {eff }}^{(\text {ex) }}$ and $S_{\text {eff }}^{(\text {so) }}$ are the exchange and spin-orbit induced asymmetries, respectively. If we were to attempt to measure the unknown polarization by a measurement of the normalized intensity asymmetry between flipping the target magnetization as suggested by equations 5.43 - 5.45, we would measure

$$
A_{m}=\frac{I_{+}^{+}-I_{-}^{+}}{I_{+}^{+}+I_{-}^{+}}=\frac{P S_{\mathrm{eff}}^{(\mathrm{ex})}}{1+P S_{\mathrm{eff}}^{(\mathrm{so})}}
$$

using equation 5.46. Note that this is not as simple as equation 5.44, and both $S_{\text {eff }}^{(\text {ex) }}$ and $S_{\text {eff }}^{\text {(so) }}$ must be simultaneously known to solve for $P$. By flipping the incident beam polarization instead, we can measure the alternative intensity asymmetries defined as

$$
\begin{aligned}
& A_{a}=\frac{I_{+}^{+}-I_{+}^{-}}{I_{+}^{+}+I_{+}^{-}}=P\left(S_{\mathrm{eff}}^{(\mathrm{ex})}+S_{\mathrm{eff}}^{(\mathrm{so})}\right) \quad \text { and } \\
& A_{b}=\frac{I_{-}^{+}-I_{-}^{-}}{I_{-}^{+}+I_{-}^{-}}=P\left(S_{\mathrm{eff}}^{(\mathrm{so})}-S_{\mathrm{eff}}^{(\mathrm{ex})}\right)
\end{aligned}
$$

Thus, by measuring all four intensities, the exchange and spin-orbit asymmetries can be isolated as

$$
\begin{aligned}
S_{\mathrm{eff}}^{(\mathrm{so})} & =\frac{1}{2 P}\left(A_{a}+A_{b}\right) \quad \text { and } \\
S_{\mathrm{eff}}^{(\mathrm{ex})} & =\frac{1}{2 P}\left(A_{a}-A_{b}\right) .
\end{aligned}
$$

This allows empirical measurement of both $S_{\mathrm{eff}}^{(\mathrm{ex})}$ and $S_{\mathrm{eff}}^{(\mathrm{so})}$ using an incident beam of known 
polarization, which is exactly how the data in Figure 5.7 was taken. Once $S_{\text {eff }}^{(e x)}$ is know, the four-intensity measurement also then allows measuring $P$ of beams of unknown polarization.

The above expressions for $S_{\mathrm{eff}}^{(\mathrm{ex})}$ and $S_{\mathrm{eff}}^{(\mathrm{so})}$ and equation 5.46 are technically only approximate. They do not account for possible coherent interference between exchange and spin-orbit effects. These effects can be qualitatively pictured as intensity due to double scattering. For example, one can measure the following asymmetry with a perfectly unpolarized incident beam:

$$
A_{u}=\frac{I_{+}^{+}+I_{+}^{-}-I_{-}^{+}-I_{-}^{-}}{I_{+}^{+}+I_{+}^{-}+I_{-}^{+}+I_{-}^{-}}=\frac{I_{+}-I_{-}}{I_{+}-I_{-}}
$$

which would be equal to zero if only equation 5.46 was considered. However, we can imagine a double scattering event where the unpolarized beam gains a polarization perpendicular to the scattering plane due to spin-orbit, and then this polarization leads to exchange asymmetries in a second scattering when the magnetization is switched. Thus we may have $A_{u} \neq 0$. As these 'double scatterings' will then be of order $S_{\mathrm{eff}}^{(\mathrm{ex})} \cdot S_{\mathrm{eff}}^{(\mathrm{so})}$, they may be only small corrections to equation 5.46 and the subsequent formulas for $S_{\mathrm{eff}}^{(\mathrm{ex})}$ and $S_{\mathrm{eff}}^{(\mathrm{so})}$.

Access to both exchange and spin-orbit effects make the 'Perpendicular' scattering geometry useful for studying the physics of the target itself. It may sometimes be preferred to eliminate spin-orbit contributions as much as possible. This is certainly true for a polarimeter which would benefit from the simplicity of only the exchange contribution. This can be achieved to different degrees by using the 'Planar' geometry of Figure 5.8(b). In this case, the plural-scattering effects of the previous paragraph go to zero for an unpolarized incident beam. The induced polarization of a primary spin-orbit scattering are only along the scattering plane normal, $\mathbf{n}$. As this is normal to the target magnetization axis, secondary exchange scatterings will not be sensitive to it, and hence no spin dependent intensities result from flipping magnetizations - i.e. $A_{u}=0$. The 'first-order' spin-orbit contributions of $S_{\text {eff }}^{\text {(so) }}$ to equation 5.46 can be removed if the incident beam polarization is oriented within the scattering plane. This is straightforward as spin-orbit asymmetries are only sensitive to polarization components out of the scattering plane. This can frequently be accomplished in polarimetry experiments that 'know' the axis of the beam polarization, and only the magnitude must be measured. For example, in photoemission from a ferromagnetic 
sample, the photoemitted electrons are assumed to be polarized along the sample (not to be confused with the polarimeter target) magnetization axis, and only the magnitude is in question. The geometric relationship between the photoemission sample and the polarimeter target scattering plane can then be organized to ensure the polarization is 'in-plane'. Some 'second-order' plural scattering effects may still remain in general. For instance, an initial spin-orbit scattering can slightly rotate the incident polarization vector in the scattering plane, ${ }^{140}$ thus changing its component along the target magnetization. This small effect can be removed completely if the incident polarization is additionally restricted to be parallel to the target magnetization, perfectly canceling these remaining effects. See refs. 95,16 and included references for additional details of disentangling exchange and spin-orbit effects.

A final note should be made about spin-orbit contributions to the scattered intensity in

either geometry. From symmetry, $S_{(\theta)}^{(\text {so })}$ must be zero for the specular reflection at normal incidence (i.e. $\theta=180$ or $\alpha=0$ in Figure 5.8). This suggests that the spin-orbit contribution should also remain quite small for slight deviations from $\alpha=0$. Although the maximum value of $S^{(\mathrm{so})}(\theta)$ approaches $\theta=180$ for high energy electrons (see plots of $S(\theta)$ for Mott scattering in ref. 140), it does not get close until well over $1 \mathrm{MeV}$ ! At lower energies, $S^{(\mathrm{so})}(\theta)$ will be quite small if $\alpha$ is kept near 0 .

Although spin-orbit and exchange effects are in general of similar strength in the $3 \mathrm{~d}$ ferromagnets and must be considered for accurate analysis of the spin-dependent scattering intensities, use of the 'Planar' geometry and near-normal incidence $(\alpha \rightarrow 0)$ can force spinorbit effects to be extremely small. A properly designed exchange polarimeter can thus minimize spin-orbit effects, allowing the simpler equations $5.43-5.45$ to be used quite accurately.

\subsubsection{More details: instrumental asymmetry}

Instrumental asymmetry was mentioned in section 5.2. All of the polarimetry techniques discussed thus far reduce the polarization measurement to a relative intensity measurement. 
This assumes that the asymmetry in the intensities being compared is only due to polarization. In realistic instruments, one must consider the possibility of asymmetry in the two intensities not related to the polarization. In Mott polarimetry, this can be due, for instance, to different efficiencies in the 'right' and 'left' electron detectors. Another source can be slight misalignment of either the detectors or the incident beam resulting in the two detectors measuring scattering at slightly different $\theta$ angles. Sources of instrumental asymmetries in Mott polarimetry and ways to deal with them are well addressed in ref. 144.

Exchange based polarimeters utilize an 'up-down' asymmetry measured with a single electron detector, thus providing relief from some of the instrumental asymmetries particular to Mott polarimetry. With only one electron detector, there are no worries about differing detector efficiencies or relative calibration. The single detector also makes alignment of the beam/target/detector unimportant as the physical alignment will be the same for both 'up' and 'down' measurements.

Care must still be taken, however, as 'effective' alignment can be susceptible to changes between the two measurements, as residual magnetic fields along the beam paths can deflect the beam. Any stray field from the magnetized target will certainly change when the magnetization is reversed. The target magnetizing coils may also alter the magnetizations of any other magnetic materials in the area. The changing field deflections can then induce asymmetry. As this type of polarimeter operates at low kinetic energy, the beam paths will be sensitive to even very small residual fields. Following the analysis of Bertacco et. al., ${ }^{169}$ this sort of instrumental asymmetry in an exchange based polarimeter can be introduced into the previous section's analysis by modifying equation 5.46 to

$$
I_{\mu}^{\sigma}=I(\theta)\left(1+\mu \sigma P S_{\mathrm{eff}}^{(\mathrm{ex})}+\sigma P S_{\mathrm{eff}}^{(\mathrm{so})}\right)\left(1+\mu A_{i}\right)
$$

where $A_{i}$ is the induced intensity asymmetry from magnetization dependent beam deflections. As in equation 5.47, we can inspect the resulting measurement of the normalized asymmetry when flipping the magnetization, now resulting in

$$
A_{m}=\frac{I_{+}^{+}-I_{-}^{+}}{I_{+}^{+}+I_{-}^{+}}=\frac{A_{i}+P\left(S_{\mathrm{eff}}^{(\mathrm{ex})}+A_{i} S_{\mathrm{eff}}^{(\mathrm{so})}\right)}{1+P\left(A_{i} S_{\mathrm{eff}}^{(\mathrm{ex})}+S_{\mathrm{eff}}^{(\mathrm{so})}\right)} .
$$


As discussed in section $5.3 .3, S_{\text {eff }}^{\text {(so) }}$ will be very negligible with appropriate scattering geometry, reducing the above expression to

$$
A_{m}=\frac{A_{i}+P S_{\mathrm{eff}}^{(\mathrm{ex})}}{1+P S_{\mathrm{eff}}^{(\mathrm{ex})} A_{i}}
$$

illustrating the desire to minimize $A_{i}$. Even if $A_{i}$ is fairly small (significantly less than 1 ), it can still present problems for measuring small beam polarizations. Taking $S_{\text {eff }}^{(\text {ex })}<1$ and $A_{i} \ll 1$, the above expression can be reduced further to

$$
A_{m}=A_{i}+P S_{\mathrm{eff}}^{(\mathrm{ex})}
$$

showing that even small instrumental asymmetries become a significant contribution when measuring small beam polarizations. The instrumental asymmetry in equation 5.53 can be systematically removed, as described in refs. $24,169,170$, if the beam polarization can be flipped allowing the four intensity measurements of section 5.3.3. This assumes, however, that there is no systematic asymmetry induced when flipping the beam polarization. In experiments such as photoemission from ferromagnetic samples, 'flipping the beam polarization' requires switching the sample (not the scattering target!) magnetization, which itself may introduce asymmetries due to photoelectron beam deflection in the sample stray fields. Certainly the most trustworthy approach for precision polarization measurements is utilizing a polarimeter with as low an instrumental asymmetry as possible.

\subsubsection{Previous exchange polarimeters}

Although not as mainstream as 'mini-Mott' polarimeters, low energy exchange-based polarimeters have been developed and utilized. Of particular note is a polarimeter developed in the Kisker group and integrated with a hemispherical analyzer for spin-resolved photoemission experiments. ${ }^{168}$ This polarimeter uses the specular reflection with $10^{\circ}$ incidence from an $\mathrm{Fe}(100)$ thin film epitaxially grown on a $\mathrm{Ag}(100)$ substrate. They claim a maximum $S_{\text {eff }}^{(e x)}$ of $-44 \%$ at about $12.5 \mathrm{eV}$ energy, with about $10 \%$ average reflectivity $\left(I / I_{0}\right)$, for a total maximum FOM of $2 \times 10^{-2}$ ! They do note that these high numbers rely on a very clean surface which ages with time and must be re-prepared fairly often. Indeed 
they discuss that the target preparation is a delicate procedure as preparations on some $\mathrm{Ag}$ substrates achieve FOM's as low as $6 \times 10^{-4}$.

A similar system was developed in the group of Ciccacci. ${ }^{170}$ It should be stressed that

because LEED in general is extremely surface sensitive, the $S_{\text {eff }}^{(e x)}$ and FOM of such a polarimeter is strongly dependent on the surface condition. As a surface reacts with residual gas and contaminants in the vacuum, these properties will degrade even in UHV. Understanding the inconvenience of working with a polarimeter whose effective Sherman function degrades rapidly with time, they investigated the use of less sensitive surfaces for use as exchange polarimeters. They found that there remained strong spin dependent effects in both the absorption ${ }^{171}$ and reflectivity ${ }^{172}$ of $\mathrm{Fe}(001)$ crystals with a $\mathrm{p}(1 \mathrm{x} 1)$ oxide overlayer, obtaining FOMs as high as $6 \times 10^{-3}$. This is appealing as the oxide surface is much less reactive and was found to maintain its effective Sherman function and FOM for weeks at a time in UHV. This target system was investigated for use as a polarimeter, ${ }^{169}$ and when coupled to a hemispherical analyzer ${ }^{170}$ the authors found a total FOM of $7 \times 10^{-4}$. The drop results from consideration of the loss of electrons between the hemisphere and the polarimeter due to a claimed mismatch of electron optical phase space acceptances. This issue will be addressed more closely in Chapter 6 .

\subsection{New exchange polarimeter}

It is obvious that the low FOM of most spin polarimeters is the limiting factor in spinresolved experiments. Compared to Mott polarimeters, the high FOM's achievable with low energy exchange-based polarimeters make them a very attractive option for high resolution spin-ARPES instrumentation. At the heart of the spin-TOF analyzer is a custom designed exchange scattering polarimeter developed completely in-house with a possible FOM as high as $1 \times 10^{-2}$. The high efficiency of the polarimeter may make it an attractive choice for other spin-resolved experiments, as well. 


\subsubsection{Target characterization}

It should be clear from the previous sections that the effective efficiency and operating conditions of such a polarimeter are strongly linked to the target system used. A method for characterizing a target system, both for searching for optimal systems and for eventual polarimeter calibration purposes, is highly desirable. The Spin-Polarized Low Energy Electron Microscope (SPLEEM) at the National Center for Electron Microscopy (NCEM) at LBL provides the ideal setup.

The SPLEEM technique is reviewed in detail in ref. 173; a diagram is shown in Figure 5.9. In short, a SPLEEM operates by focusing a spin polarized electron beam onto a sample surface at normal incidence and images the specular reflected beam. A single image taken in this way provides a topological image. The spin polarized source is based on the popular GaAs photocathode ${ }^{92,93,94}$ and can easily reverse its polarization optically by reversing the helicity of the excitation laser. A second image taken with the opposite polarization gives equivalent topological information, but when the two images are subtracted the topological information exactly cancels. What remains is the pure exchange asymmetry of the sample reflectivity, proportional to $\mathbf{P} \cdot \mathbf{M}$, where $\mathbf{P}$ is the incident polarization and $\mathbf{M}$ is the sample magnetization. As mentioned in section 5.3.3, the symmetry of the normal incidence/reflection geometry removes the possibility of any spin-orbit effects. Thus, the SPLEEM can take direct microscopic images of a sample's magnetic domain structure. Magneto- and electro-static optics downstream of the electron gun allow complete control of the polarization vector in all three spatial dimensions, allowing these images to be taken with respect to arbitrary sample magnetization directions.

SPLEEM essentially images the $S_{\text {eff }}^{(e x)}$ of the target with high spatial resolution, providing a perfect tool for investigating polarimeter target candidates. For polarimetry applications, the spatial resolution provided by SPLEEM is not entirely necessary, and the total intensity of each image can be integrated. Another strength of the SPLEEM is, however, very useful: its data acquisition speed. Images with sufficient statistics for determining $S_{\text {eff }}^{(e x)}$ are obtained at close to video rates. This allows data to be taken in a high efficiency 
(a)

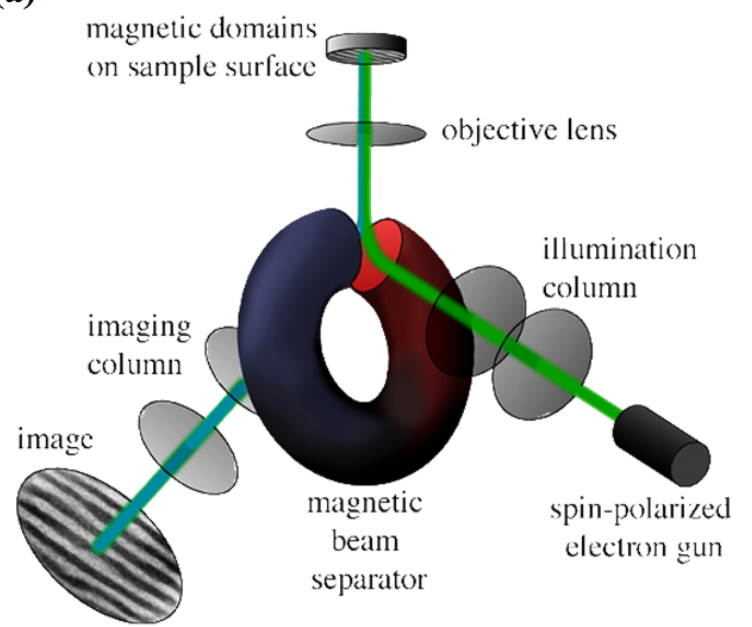

(b)

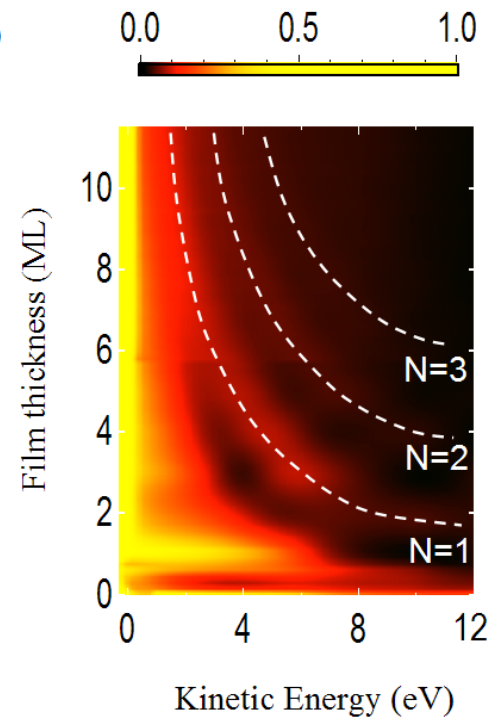

Figure 5.9. (a) Cartoon diagram of SPLEEM operation, imaging a sample's spin-dependent, normal incidence, low energy reflectivity. Figure courtesy of Andreas Schmid. (b) $\mathrm{Co} / \mathrm{W}(110)$ reflectivity as a function of incident energy and Co thickness. Figure adapted from ref. 174. See text for details.

spectroscopic mode, where the incident kinetic energy is scanned through a certain window, quickly determining $S_{\text {eff }}^{(e x)}$ as a function of energy - vital for determining the optimal operating characteristics of a polarimeter. The efficiency of the SPLEEM spectroscopy mode allows taking data during constant sample 'growth'. For instance, data can be taken while material is being epitaxially evaporated onto the sample substrate allowing 2 dimensional phase space maps of $S_{\text {eff }}^{(e x)}$ as a function of energy and target thickness. As we will see, this is very convenient.

For initial work, two systems were studied: ultra-thin Fe(110) films grown on a W(110) crystal, and ultra-thin $\mathrm{Co}(0001)$ films also grown on W(110). Fe and Co are good candidates for the polarimeter in general as they have large exchange splitting which would be expected to lead to large exchange Sherman functions. Bulk crystals of either are not great options as clean surfaces can be difficult to prepare (many cycles of sputtering and annealing). Additionally they require large external fields to magnetize, and once they are, the large stray magnetic fields will lead to problems with deflection of the low energy electrons and instrumental asymmetry. As noted in refs. 169,168, thin films can easily be fully magnetized 
in a single domain and have minimal stray fields. In particular, a thin film with in-plane magnetization gives extremely low external fields.

The W(110) crystal was chosen as a substrate for a couple of reasons. Tungsten surfaces have a relatively straightforward recipe for reliable in-situ cleaning; annealing cycles in $3 \times 10^{-8}$ torr of oxygen, followed by flashes to $2000^{\circ} \mathrm{C}$. Anytime the target needs to be freshly prepared, the W crystal can simply be flashed to start from scratch. The (110) substrate surface was chosen because the two-fold symmetry results in magnetic anisotropy with a single magnetic 'easy-axis' along the substrate $[1, \overline{1}, 0]$ direction for films under $\sim 60$ A. ${ }^{175,176,177}$ This ensures reliable and complete switching of the magnetization direction along the correct axis. Good descriptions of the epitaxial growth characteristics of Fe and Co on W(110) are contained in ref. 177

Both systems showed promising results which were fully published by Graf et. al. in ref. 174. As the Co/W(110) system showed slightly better results and was ultimately chosen for initial work in the polarimeter, we will give a brief summary here of the Co results only. A false-color 2D phase-space plot of average reflectivity of the $\mathrm{Co} / \mathrm{W}(110)$ system is shown in Figure 5.9. The reflectivity is averaged over a flat-terrace area of the crystal within a single magnetic domain. The energy independent, horizontal 'corrugations' in the plot are a result of slight reflectivity peaks when full monolayers of Co are completed. This periodicity is in fact what is used to calibrate the growth rate (i.e. the scaling of the vertical axis). There is also an obvious pattern of curves marked by the white dotted lines as guides to the eye. This is due to a Quantum Size Effect (QSE) characteristic of ultra-thin films, ${ }^{178}$ which was hoped to increase exchange asymmetry above bulk values for improved polarimeter performance. ${ }^{179}$

The QSE can be pictured like a Fabry-Pérot-like interferometer as diagrammed in Figure 5.10. Electrons incident on the thin film along the surface normal with kinetic energy $E$ enter the thin film by coupling to Bloch states with $k$-values determined by the thin film electronic bandstructure, $E\left(k_{z}\right)$. Reflectivity is enhanced when standing waves are perfectly formed within the thickness of the thin film, meeting the constructive interference condition 


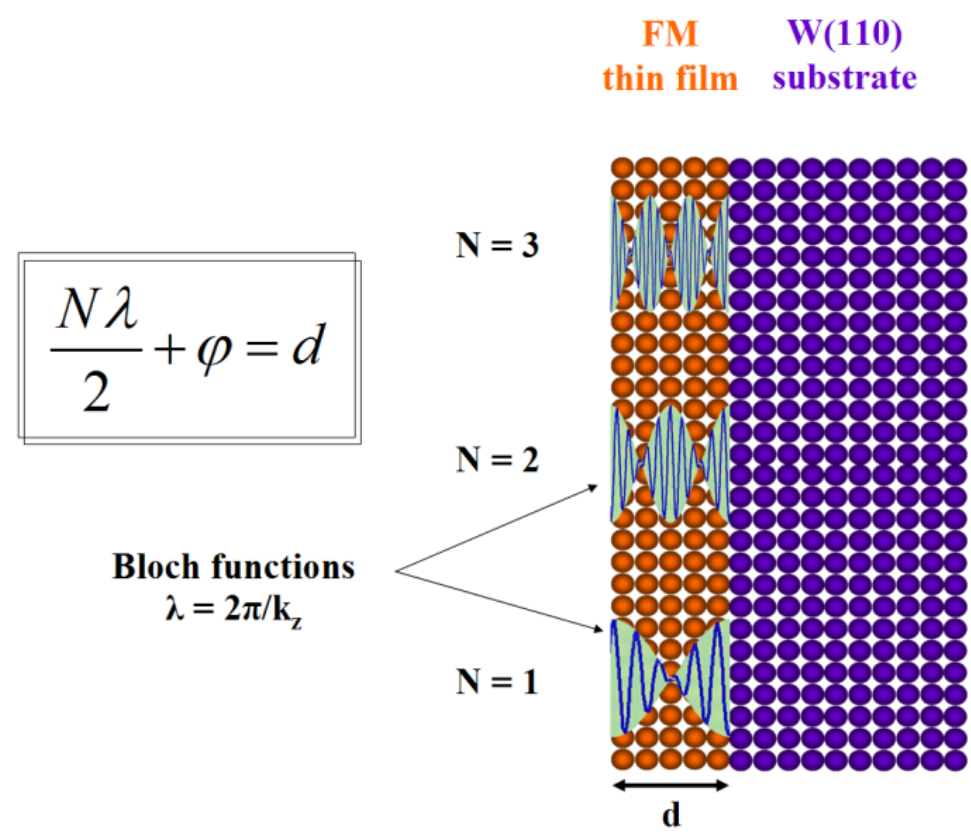

Figure 5.10. Quantum Size Effect (QSE). Reflectivity boosted at points of constructive interference of Bloch states.

of

$$
\frac{N \lambda}{2}+\phi=d
$$

where $\lambda=2 \pi / k_{z}$ is the Bloch state wavelength, $\phi$ is a constant phase shift at the interfaces, $d$ is the film thickness, and $\mathrm{N}$ is an integer. The enhanced reflectivity standing wave states of $N=1,2,3$ are shown in the figure. The curves of increased reflectivity in the plot of Figure 5.9(b) provide a vivid illustration of the existence of these quantum standing waves.

Figure 5.11 shows several constant thickness (horizontal) 'cuts' from the reflectivity plot in Figure 5.9(b) for both spin directions with respect to the Co magnetization axis. The periodic oscillations with respect to energy in each plot is again a clear manifestation of the QSE interference. Two spin-dependent features of the oscillations immediately jump out. First, the damped amplitude of the minority direction oscillations compared to the majority can be interpreted as due to a smaller inelastic mean free path of minority electrons. ${ }^{174}$ Second, the minority spin oscillations are clearly phase shifted to higher energy with respect to majority spins. This is easily explained by the exchange split bandstructure and the QSE expressed in the constructive interference condition in equation 5.57. For a given film 


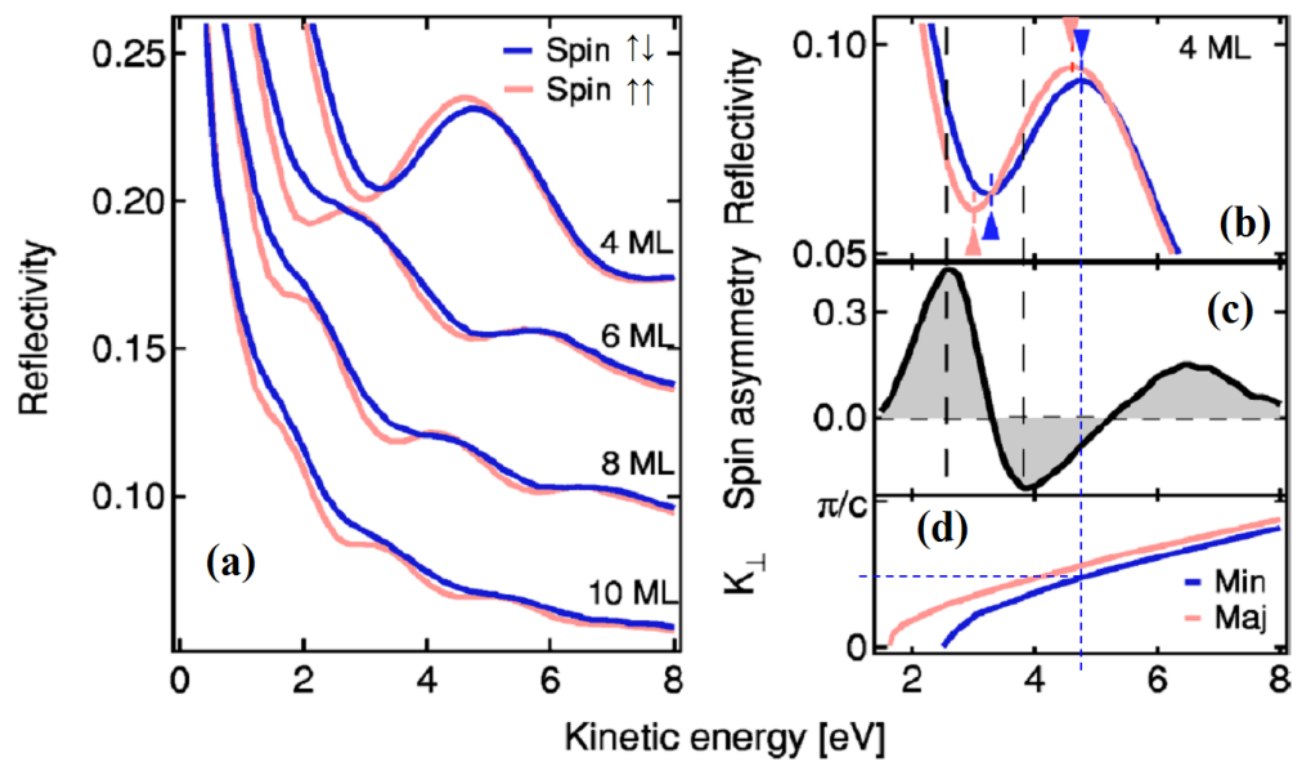

Figure 5.11. Figure taken from ref. 174. (a) Reflectivity as a function of incident energy with polarization parallel (antiparallel) to the magnetized Co majority (minority) spins in red (blue) for several film thicknesses. (b) Expanded view at 4 monolayer thickness. (c) $S_{\text {eff }}^{(e x)}$ obtained from (b). (d) Calculated Co bandstructure along the out-of-plane direction (from ref 178).

thickness, only precise $\lambda$ values lead to perfect standing waves. Because the bandstructure is exchange split (as shown in Figure 5.11(d)), the precise $\lambda$ values are obtained at different energies for the different spin directions. The spin-dependent reflectivity spectra of panel (a) and the included QSE oscillations qualitatively agree quite well with the previous theory and experiments found in ref. 178 .

As discussed and performed by Zdyb and Bauer ${ }^{179}$, the oscillation peak (and minimum) locations at each thickness, along with equation 5.57 can be used to experimentally map the unoccupied bandstructure of the thin film. For instance, the first QSE peak in the 4 monolayer (ML) minority reflectivity curve in Figure 5.11(b), corresponding to $N=1$ in equation 5.57, occurs at the energy marked by the vertical blue dotted line. As there are 2 Co basal planes per unit cell, $4 \mathrm{ML}$ corresponds to a thickness of $2 \mathrm{c}$, where $\mathrm{c}$ is the lattice constant along the $z$-axis (perpendicular to the plane). With $d=2 c$ and $N=1$, equation 5.57 gives $\lambda=4 c$ (if we ignore the phase shift as a small correction), which is equivalent to $k_{z}=\pi / 2 c$. This value of $k_{z}$ and $E$ indeed corresponds to the $k_{z}$ bandstructure 
as shown by the intersection of the blue dotted lines agreeing perfectly with the calculated minority bandstructure in panel $(\mathrm{d})$.

More important for polarimeter development is the exchange asymmetry, $S_{\mathrm{eff}}^{(e x)}$, shown in Figure 5.11(c) as derived from the experimental reflectivities of panel (b). The slight phase shift of the QSE oscillations leads to the strong peaks in asymmetry, which may be used for a high performance polarimeter. The first asymmetry peak at $\sim 2 \mathrm{eV}$ may be additionally enhanced due to the spin-dependent bandgap (panel (d)), as described in section 5.3.2. The full reflectivity data sets in the $2 \mathrm{D}$ thickness and energy phase space (Figure 5.9(b) and left of Figure 5.12) are easily converted point-by-point to the effective Sherman function plot in the center of Figure 5.12 according to

$$
S_{\text {eff }}^{(e x)}=\frac{1}{P} \frac{R_{\text {min }}-R_{\text {maj }}}{R_{\text {min }}+R_{\text {maj }}}
$$

where $R_{\min (\mathrm{maj})}$ is the reflectivity measured with polarization parallel to the Co minority (majority) direction and $P$ is the polarization magnitude of the incident electron beam. The QSE enhancement is visible as the distinct oscillations in the $S_{\text {eff }}^{(e x)}$ plot, leading to values as high as over $40 \%$. The distinct lack of any asymmetry below 2 ML results from the fact that the Curie temperature for these thickness is below room temperature at which the data was taken. This plot can be further converted point-by-point to the FOM map shown on the right according to the usual formula. Thus a maximum FOM of $2 \times 10^{-2}$ is found at a kinetic energy of $\sim 2 \mathrm{eV}$ with a Co thickness of 5 ML.

This research has shown that ultra-thin Co/W(110) targets are promising candidates for use as a spin polarimeter with vastly improved efficiency over standard Mott polarimeters. These extreme FOM values are due to the QSE. Unfortunately, this causes the $S_{\text {eff }}^{(e x)}$ and FOM to be strong functions of kinetic energy. This can complicate data analysis if the polarimeter is to be used with a simultaneous range of incident energies, as would be the case in a TOF scheme. It may prove to be easier to operate with thicker films and higher kinetic energies. Although they have somewhat lower FOM's, they are less strongly energy dependent, relieving some of this difficulty. Most importantly, the current work has shown that the LBL SPLEEM is an excellent instrument for continuing investigations of various scattering targets and calibrating their characteristics. Additionally, the completed 


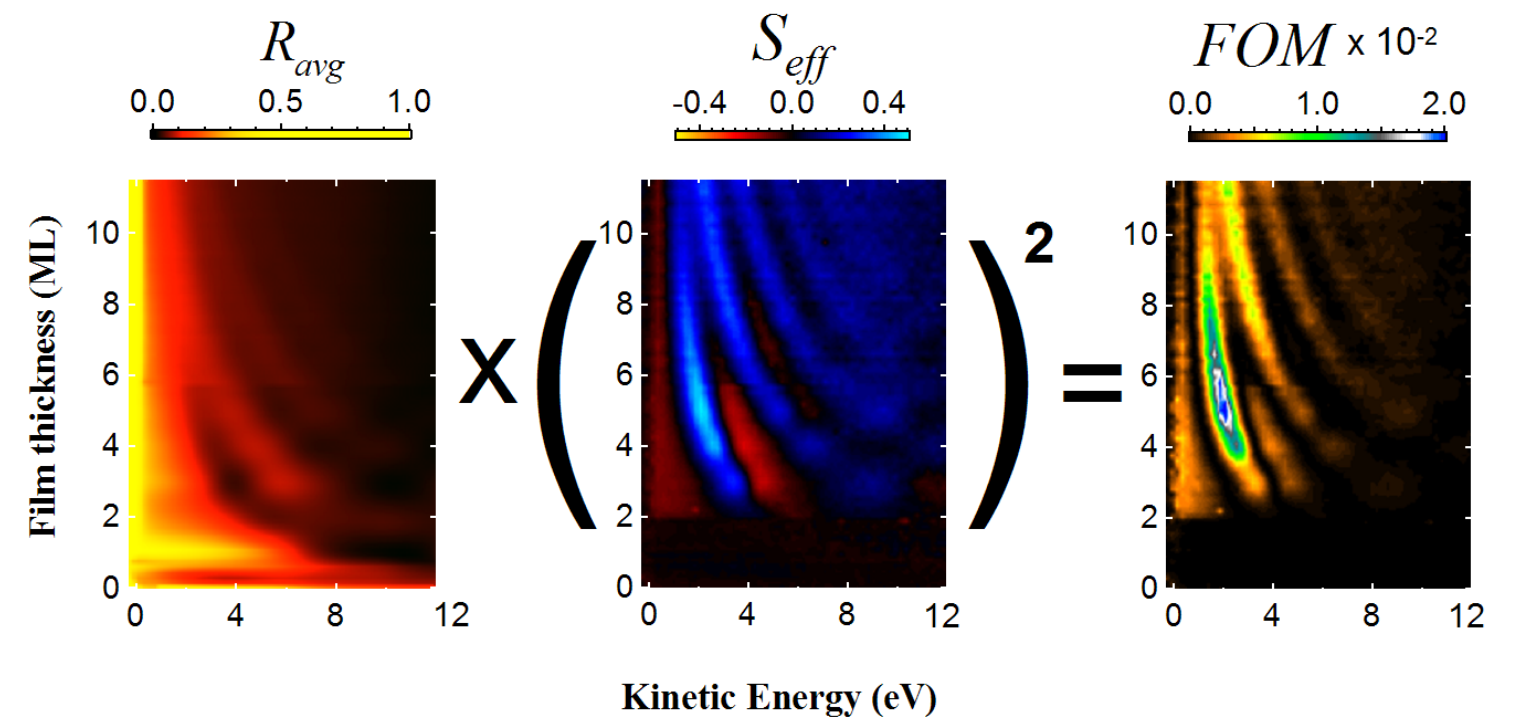

Figure 5.12. Data taken from ref. 174. Reflectivity plot on the far left, and the derived Sherman function and FOM plots on the right.

SPLEEM work has shown that Fe and Co films on W(110) are indeed a great starting point for target development.

\subsubsection{Scattering geometry and detection}

The essential ingredients to our new low energy exchange polarimeter can be summarized as: (1) an electron detector, (2) a single crystal scattering target, and (3) the geometric relationship between them defining the scattering geometry. These three ingredients are illustrated to scale in Figure 5.13, with the scattering geometry diagrammed in the inset.

The scattering geometry is chosen to optimize performance. Peak effective Sherman function and FOM are generally found at normal incidence, ${ }^{171}$ and so the polarimeter is designed to operate as close to perfect backscattering as possible. As discussed in section 5.3.3, complicating spin-orbit effects are also kept to a minimum by operating near normal incidence. The substrate $\mathrm{W}$ crystal axes are oriented as shown to put the magnetization axis of the thin film target in the scattering plane for the 'Planar' geometry, giving further reduction of any spin-orbit effects that could lead to asymmetric intensities even 


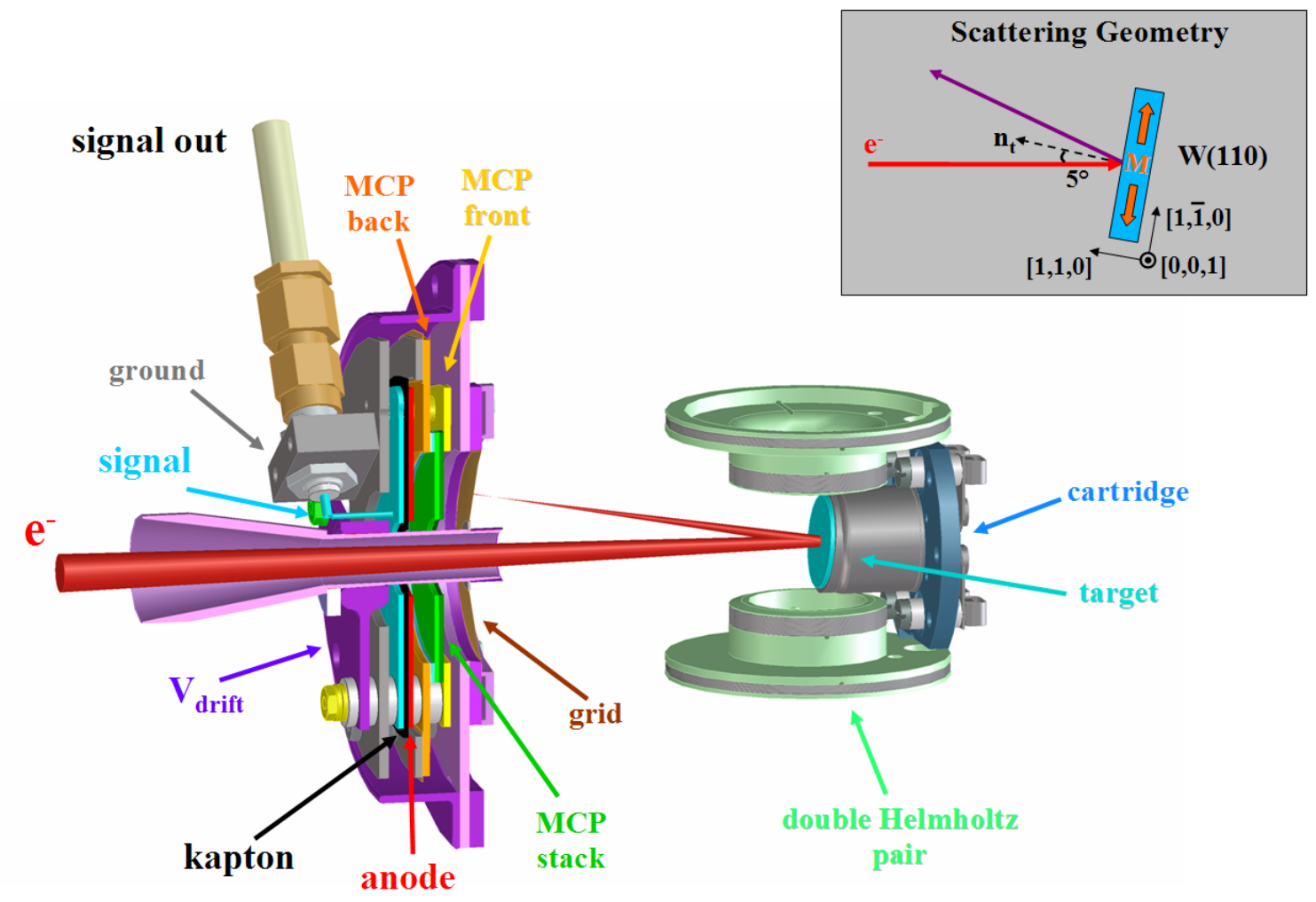

Figure 5.13. Scale illustration of polarimeter's scattering/detection scheme. Details in text. Scattering geometry and crystal orientation shown in inset.

with an unpolarized beam. From section 5.3.3, if the incident beam's polarization can be arranged to be in this scattering plane, virtually all spin-orbit effects can be fully neglected.

The electron detector design was carefully considered to fulfill its numerous requirements. As the majority of the backscattered electrons are done so elastically (little change or loss of kinetic energy), the polarimeter technique is in principle consistent with simultaneous TOF energy analysis (Chapter 4). For seamless integration into a TOF energy analyzer, the detector must have extremely good timing resolution. An MCP-based scheme was chosen because of their excellent timing characteristics, detailed in section 4.4.1.

The near-perfect backscattering places restrictions on the geometry of the MCP detector; the outer edge of a typical MCP assembly would require a large enough scattering angle so as not to block the incoming beam. To achieve as small a backscattering angle as possible, an annular detector was designed with a central hole through which the incoming 
beam could pass on its way to the target. After scattering through a minimal angle, the beam would then strike the front of the detector's active area and be detected. MCPs (chevron pair) with a $27 \mathrm{~mm}$ active outer diameter and a $10 \mathrm{~mm}$ central hole ('dead-space' extends to $12 \mathrm{~mm}$ diameter) were purchased as part of a full detector assembly, the Hamamatsu F4294-09. However, the assembly did not provide for the required high voltage on the anode or the onboard signal decoupling as did the Hamamatsu F4655-12 (described in section 4.4.1 and used for the spin-integrated leg of the TOF analyzer). Thus, the MCPs were cannibalized from the assembly and installed into the home-built detector assembly shown in cross section in Figure 5.13. The incoming beam passes through a $6 \mathrm{~mm}$ minimum diameter tube through the entire detector. The final geometry then allows passage of incoming electrons out to a radius of $3 \mathrm{~mm}$ from the central optical axis while giving detection at a radius as small as $6 \mathrm{~mm}$. With the scattering target $50 \mathrm{~mm}$ from the MCP, this allows the scattering to comfortably take place at less than $5^{\circ}$ from normal while still accepting a usable beam width.

It may be imagined that the spatial/angular distribution in the incident beam could be resolved in a multichannel detector after the scattering process. A multichannel detector could then be ambitiously imagined to give simultaneous channels of angle resolution in an ARPES experiment, for instance. The additional complexities, however, were deemed far to extensive and problematic, and a single-spatial-channel approach was established early in the design process.

The homemade detector assembly incorporates the aforementioned MCP chevron pair, a grid, and a single-plate anode. The anode is separated from a second plate by a thin Kapton sheet, forming a $\sim 300 \mathrm{pF}$ capacitor allowing immediate decoupling of the signal from the high voltage of the anode. The signal is somewhat ungracefully taken from this second disk by a thin strip of tantalum spot welded to the disk, and then fed into an in-vacuum coaxial cable to a double-sided SMA-type electrical feedthru. The MCPs, anode, signal output, and signal cable shielding are all kept electrically isolated from all the purple-colored parts in the figure, which along with the grid, the target itself, and a 'Faraday cage' (not shown) around the target, forms a field-free drift region for the scattering. The all- titanium, alumina, 

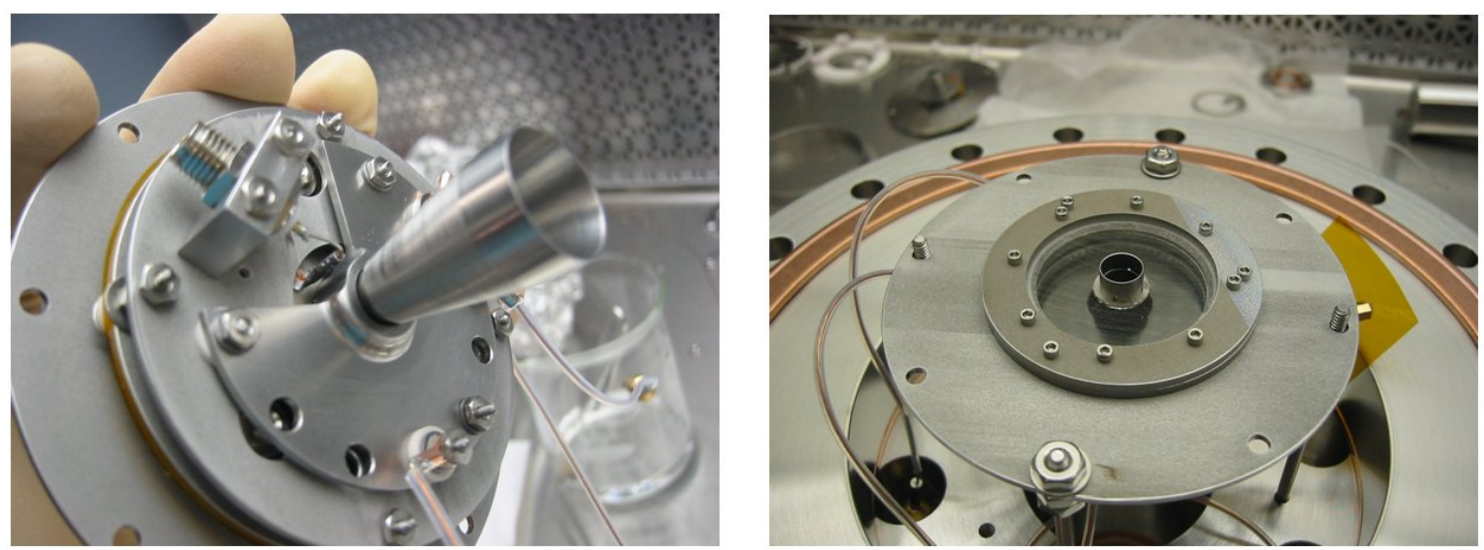

Figure 5.14. Photographs of the home-built MCP detector assembly. Left: the upstream, rear side of the detector, showing the beam input 'funnel' and the SMA signal output connection. Right: front active side of the detector, showing MCPs, grid, and central tube extending through the grid.

and Kapton construction of the assembly allows it to be heated to regular UHV bakeout temperatures, unlike the Hamamatsu F4655-12 (section 4.4.1). Photos of the assembled detector unit before installation are shown in Figure 5.14.

The operation of the polarimeter in a TOF scheme requires the detector assembly to give excellent timing resolution in conjunction with the same timing electronics as described in section 4.4.2. Before the unit was installed into the polarimeter, it was temporarily mounted in vacuum for testing of its signal speed in a manner identical to that of the Hamamatsu F4655-12 described at the end of section 4.4.1. A typical output signal pulse as recorded with an oscilloscope is shown in Figure 5.15(a). The 525 ps rise time is similar to the 414 ps of the commercial unit from Figure 4.5. The rise time is likely slightly longer due to larger size of the MCP and its unique geometry in addition to the larger capacitance. The $5-10 \mathrm{mV}$ amplitude of the current assembly is however significantly smaller than the $50 \mathrm{mV}$. This is likely due to the larger capacitance of the homemade signal output, as well as a less thorough coupling to the actual signal cable. The total timing resolution achieved in conjunction with the signal processing electronics was also tested as described in section 4.4.2. The timing resolution of the present unit is shown in blue in Figure 5.15(b), directly compared to the results of the commercial unit in red (previously shown in Figure 4.7). As discussed in section 4.4.2, one of the major contributions to timing jitter is the range of distances between 

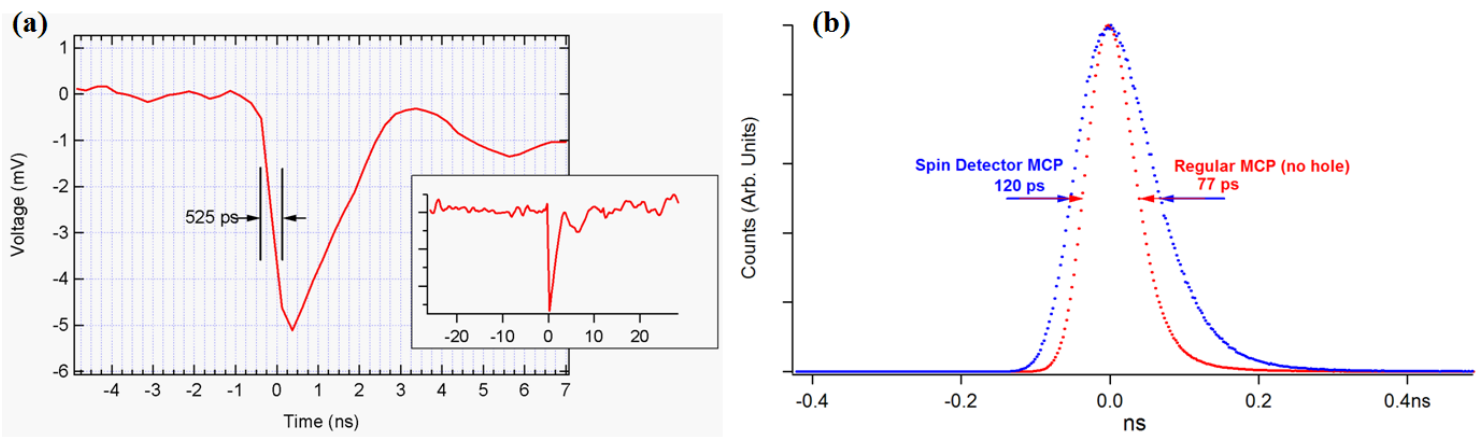

Figure 5.15. Timing characteristics of the polarimeter's annular MCP detector assembly. (a) Detector output signal recorded on an oscilloscope, as in Figure 4.5. (b) Timing resolution obtained by laser excitation and TOF signal processing electronics. Annular detector in blue, compared to the smaller, regular commercial unit of Figure 4.7 in red.

the location of the count arrival at the $\mathrm{MCP} /$ anode and the location of the signal output wire, combined with finite signal propagation times along the anode. Thus the current unit should be expected to give degraded timing resolution due to its larger diameter and the presence of a hole in the center of the anode, causing a larger spread in signal propagation times. The 120 ps resolution of the present unit is still quite satisfactory for the present needs of the TOF analyzer, and will likely not be the limiting factor in the final total energy resolution of the instrument. Although the small signal amplitudes can be tricky to deal with, the total achieved performance did not call for a rebuild of the assembly. If it is decided that improved performance is necessary, a smaller output capacitance and a more thorough signal plate-to-signal wire connection would be initial points which could be improved.

Integration into a TOF scheme also requires that the possible trajectories introduce minimal variations to the total flight path length (see section 4.1). Straightforward attention to the geometry of the specular reflection should be sufficient. With a large accelerating voltage between the grid and the front of the MCP, the flight time from one to the other becomes negligible, and we can consider the path lengths only to the grid. If the incoming beam is focused to a point $\sim 50 \mathrm{~mm}$ behind the scattering target, the scattered beam will then be focused to a single point on the grid, and the path length variation within the polarimeter becomes minimal. However, if the focal point is closer (or farther), the spot on 
the grid will be larger. If the grid was flat and parallel to the actual MCP, this can result in a more significant path length variation because of the slight angle away from normal incidence backscattering. The grid can be shaped to correct for this if it is angled such that it is normal to the scattered beam. In the present scheme, the grid (made of a 100 lines/inch woven 0.001 " tungsten wire mesh with $81 \%$ open area transmission) is strectched into a concave cone, as illustrated in Figure 5.13, to approximate this correction at any rotation of the scattering plane about the incident optical axis. This way, a range of focal distances can be tolerated.

\subsubsection{Full polarimeter layout}

The full design of the polarimeter is shown in a scale cross section view in Figure 5.16. The polarimeter is attached via the main mounting flange to the output of the TOF electrostatic lens system (Chapter 4) which focuses its exiting electron beam into the polarimeter through the detector tube as shown. As the scattering can take place at very low kinetic energy $(2-10 \mathrm{eV})$, the polarimeter must be well shielded, and so the scattering area is contained within two layers of $\mu$-metal (see section 4.5.2). Penetrations through the shielding are minimized, and the penetrations that are required are fully 'sleeved' to minimize the entrance of external fields. During assembly, the residual field inside the shield was measured as $0.1-0.2 \mathrm{mG}$. The scattering target is quite surface sensitive, and so vacuum pressure must be kept to a minimum around the target. This is difficult to achieve as the target is located in the center of three concentric cylinders (two magnetic shields, and one 'Faraday cage' defining the potential of the field-free drift space), making it difficult to get pumping to where it is needed. Pumping is provided by vacuum in the TOF analyzer directly upstream, as well as from a turbo pump attached to one of the polarimeter's radial flanges (not shown). An extra flange is provided for further pumping, but it is unknown how much it will be able to effect the pressure at the target surface. It will be important to monitor

whether vacuum pressure at the target surface is an issue: initial testing has been rather positive and will be discussed in Chapter 6 .

The surface sensitivity of the exchange scattering requires a clean target to be prepared 


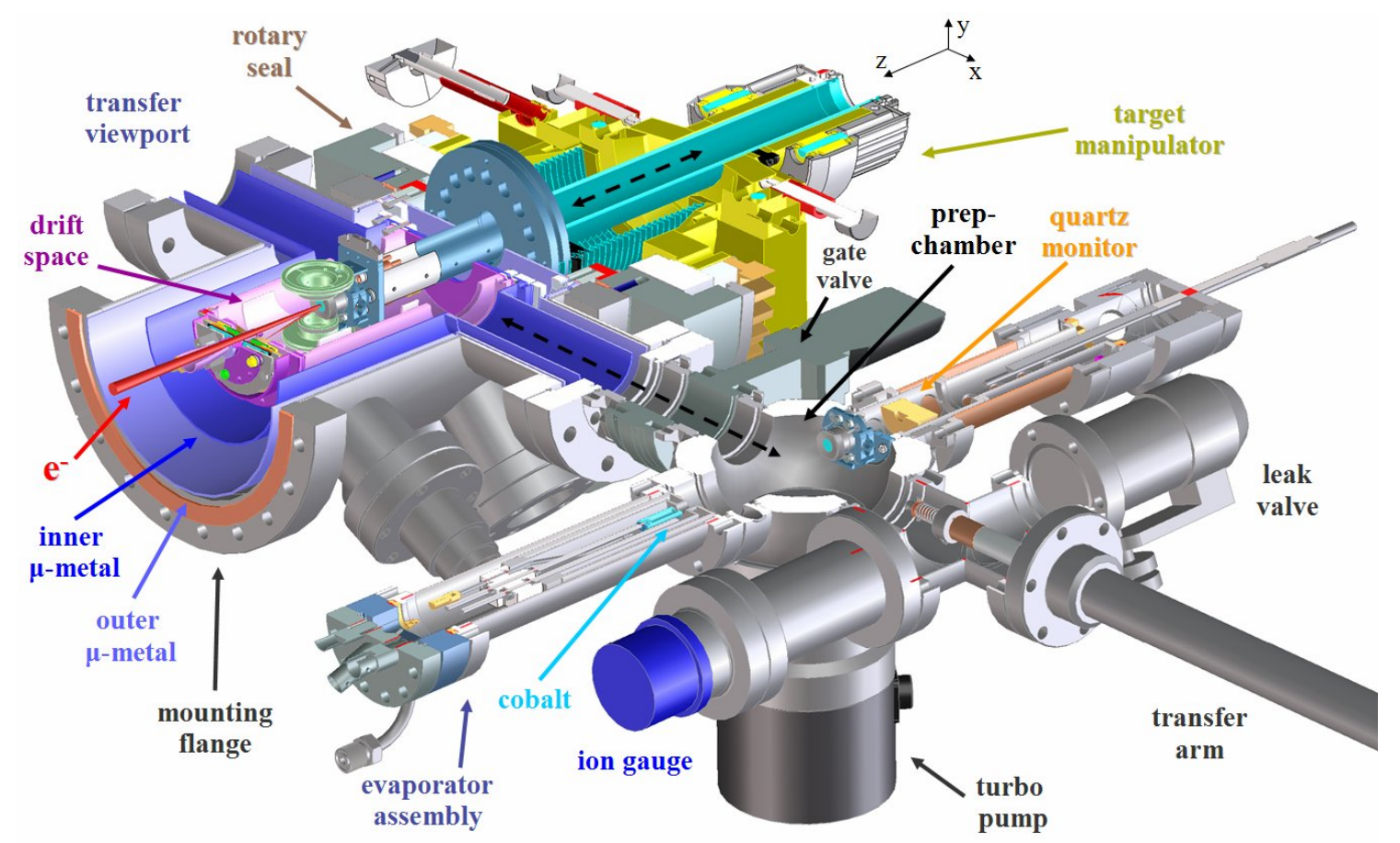

Figure 5.16. Cross sectional view of the complete spin polarimeter, to scale.

in-situ. This is performed in an adjacent 'prep' chamber, on the lower right of Figure 5.16. The prep chamber is equipped with all the tools required to clean and prepare the target. The cleaning recipe for the $\mathrm{W}$ substrate utilizes high temperature flashing in a $3 \times 10^{-8}$ partial pressure of oxygen. The prep chamber is supplied with a stage for the required e-beam heating of the crystal, a leak valve for $\mathrm{O}_{2}$ introduction, and a gate valve to isolate its vacuum from the shared vacuum space of the rest of the polarimeter, TOF analyzer, and ARPES analysis chamber. Opposite the target stage is an e-beam evaporator with a watercooled jacket for UHV deposition of the magnetic thin films (presently cobalt). If the target is removed from the heating stage, flux from the evaporator passes through a hole in the center of the stage to a quartz crystal growth monitor for calibrating the evaporation rate. Once a rate is firmly established, the target is returned to the heater stage for a deposition of precise thickness. When target preparation is complete, the target is transfered into the polarimeter on its analysis stage via the magnetically-coupled transfer arm shown on the far right. Photographs of the innards of the e-beam evaporator and the integrated heater stage and growth monitor are shown in Figure 5.17. 

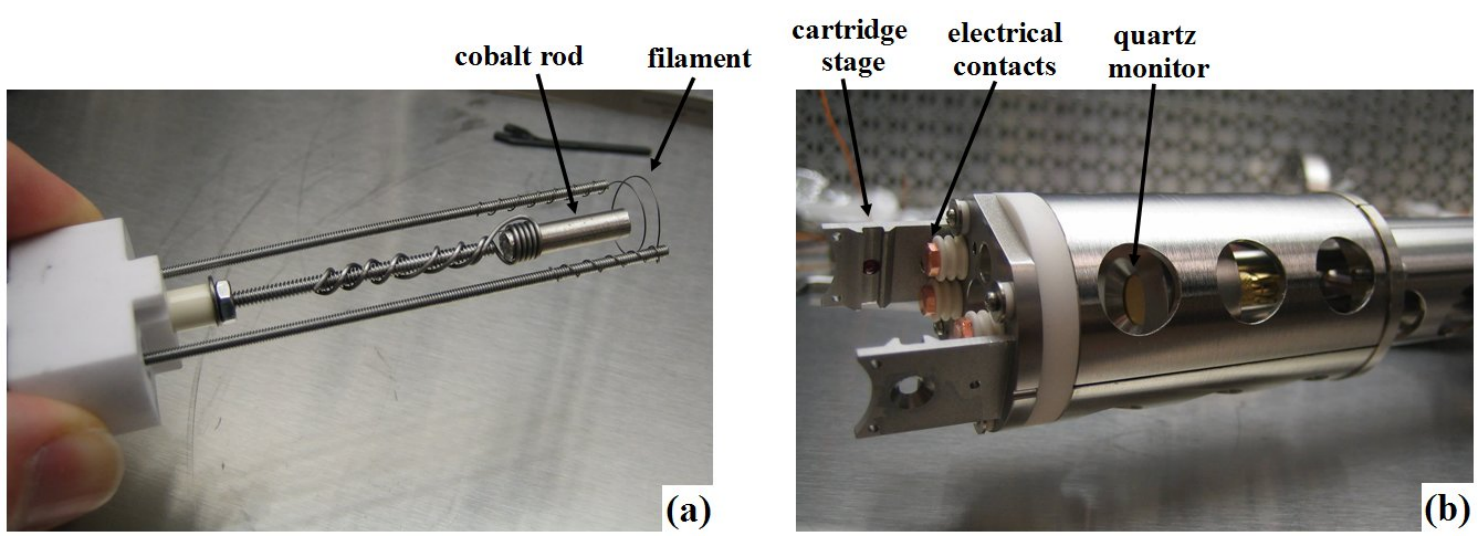

Figure 5.17. (a) Central components of the Co e-beam evaporator. (b) Integrated heater stage and growth monitor.

To minimize the total size and weight of the polarimeter, it was attempted to keep the prep chamber as small and efficient as possible. The chamber has the absolute minimum number of ports required. Pumping was initially supplied by a $70 \mathrm{l} / \mathrm{s}$ turbo pump directly attached to the central chamber on a 4.5" flange. Unfortunately, the ultimate base pressures would only get into the upper $10^{-10}$ torr range, and would take a significant amount of time to recover after the high-temperature $\mathrm{W}$ flashing and thin film evaporations. Changing to a 300 l/s turbo pump on a 6" flange, mounted through a 4.5-6" conical adapter has given much better pressures during target preparation, with a base pressure of $2-3 \times 10^{-10}$ torr. Further improvements, especially during heating and evaporating, would likely require a larger chamber volume to accommodate more pumping speed, thus sacrificing economy. For now, the performance is sufficient for preparing effective targets.

\subsubsection{Target manipulation and magnetization}

Manipulating the target's position and magnetization is central to the operation of the polarimeter. To enable easy transfer between the analysis position in the polarimeter chamber and the prep chamber, the substrate crystal is mounted into a sample cartridge based on the popular system from the commercial electron microscope company 'Elmitec'. The same type of cartridge is used at the NCEM SPLEEM as well as the PEEM2 microscope at the ALS. The cartridge contains an internal filament for e-beam heating, and electrical 

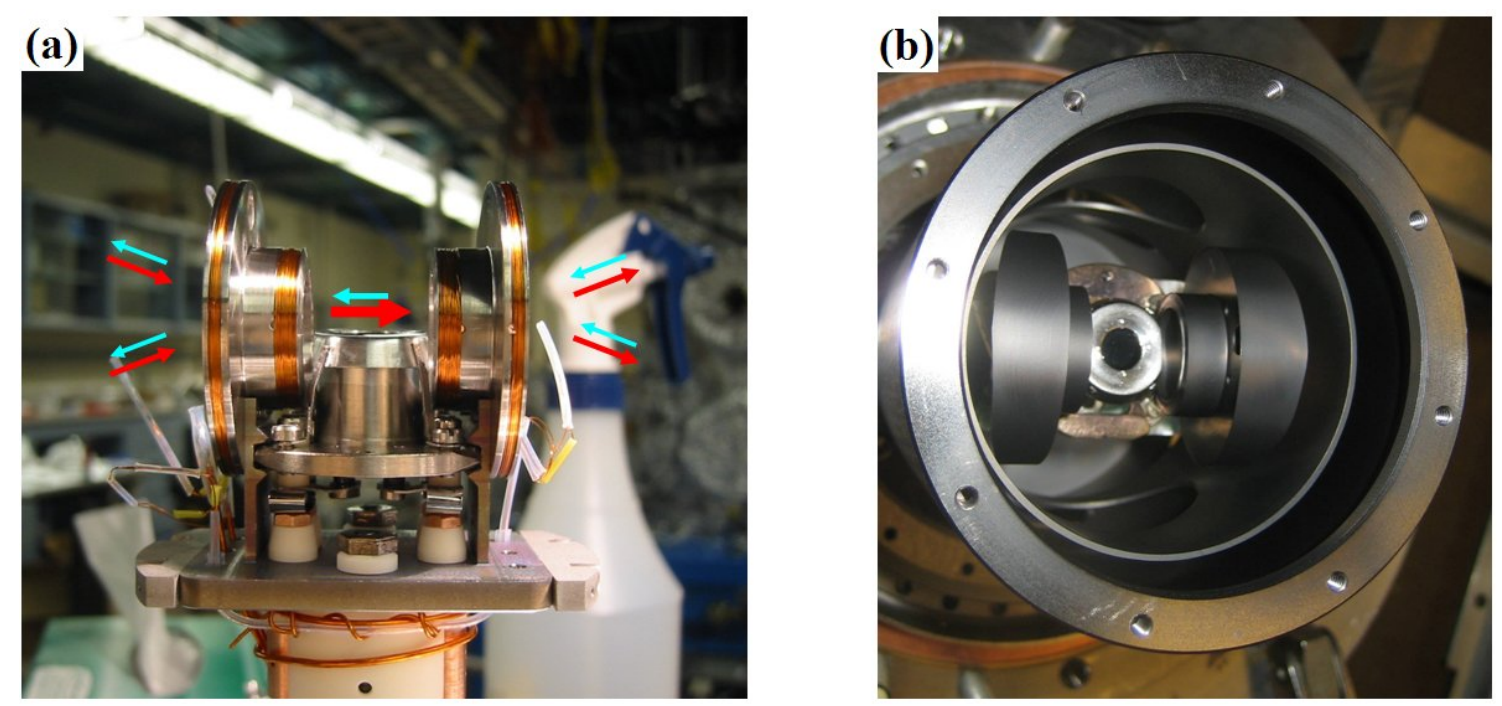

Figure 5.18. (a) Photograph of polarimeter analysis stage and double Helmholtz magnet assembly with target cartridge in place. Red arrows represent the field from the inner Helmholtz pair, blue arrows represent the field from the outer Helmholtz pair, illustrating the strong net field at the target surface and the far-field cancellation.

contact for supplying current is made through 'sled'-shaped sliding contacts. Transferring the cartridge uses a clever lock-and-key design, allowing operation with a single-magnet transfer arm.

Target magnetization is performed by Helmholtz coils centered on the analysis stage for rapid magnetization reversal during measurement. The coil design required small size to be able to fit in the analysis position, and yet also had to be designed to have minimal stray fields. Although all the parts internal to the magnetic shielding were made from strictly non-magnetic materials, magnetizing the $\mu$-metal shielding itself was a concern. To keep instrumental asymmetry to a minimum, the magnet coils must provide a large enough local field at the target to fully switch its magnetization, but must fall off rapidly with distance so as not to influence the magnetization of the inner $\mu$-metal layer. The final design is illustrated in Figure 5.18(a), showing the target cartridge in place between a nested set of Helmholtz pairs. A Helmholtz pair is two equal concentric coils separated by a distance equal to their radii, a configuration which gives a fairly homogeneous field near the center of the pair directed along the axis between them. The inner pair of coils has 160 windings each, while the outer pair has only 40 windings each, wound in the opposite direction, with 
twice the radius. Each inner coil is powered in series with each outer coil, so every winding receives the identical current, $I$. The field at the center of the coils (at the target surface, along the coil's axis) due to the inner pair is then

$$
B_{i}=\frac{8}{5^{3 / 2}} \frac{\mu_{0}(160 I)}{R}
$$

where $\mu_{0}$ is the permeability of vacuum and $R$ is the radius of the inner coils. The field at the center due to the opposing outer pair is then

$$
B_{o}=-\frac{8}{5^{3 / 2}} \frac{\mu_{0}(40 I)}{2 R}=-\frac{1}{8} B_{i}
$$

resulting in a combined field at the target of

$$
B_{t}=\frac{7}{5^{3 / 2}} \frac{160 \mu_{0} I}{R}
$$

So in the center of the coils, the field from the inner pair vastly outweighs the countering outer pair, resulting in a strong net central field. However, away from the coils is a different story. In the 'far-field', we can approximate the field due to each individual coil as that due to a perfect dipole, $m$, of magnitude $m=C A$ where $\mathrm{C}$ is the total current in the current loop, and A is the area enclosed by the loop. The 'far-field' of a dipole scales as $m / r^{3}$, where $r$ is the distance away from the dipole. Thus, the field far from each inner coil can be approximated as

$$
B_{\mathrm{ff} i} \propto \frac{160 I \pi R^{2}}{r^{3}}
$$

while that of each outer coil is approximated as

$$
B_{\mathrm{ffo}} \propto-\frac{40 I \pi(2 R)^{2}}{r^{3}}=-B_{\mathrm{ffi} i}
$$

We see that the nested set of Helmholtz pairs exactly cancels each other in the far-field dipole approximation! In this way, the compact magnet system can produce a strong local field for target magnetization reversal, and yet have small stray-fields which could risk causing instrumental asymmetry. The fields due to each Helmholtz pair are depicted by the arrows in Figure 5.18, illustrating the net central field and the far-field cancellation.

It was determined via domain imaging in the SPLEEM that a field of $66 \mathrm{G}$ was enough to completely magnetize and flip the magnetization of the thin film targets. With a test 
current of $I=0.5 \mathrm{~A}$ in the above equations, the field at the target position was measured as $\sim 35 \mathrm{G}$. With the inner coil having an average radius of $9.32 \mathrm{~mm}$, equation 5.61 predicts a field of $67 \mathrm{G}$; the difference is likely due to the coils not being in the exact Helmholtz geometry. When measuring the magnetic field at the distance of where the $\mu$-metal would be, no difference in field could be detected with the test current on and off. Just to be sure of complete magnetization, the polarimeter standardly operates the coil with $I=1.7$ A for a field of $119 \mathrm{G}$ at the target surface. As the polarimeter cannot operate while the coils are energized, the coils are pulsed between measurements. The wire used is AWG 36 (0.005" dia. conductor) with a single build of Kapton insulation (total 0.0056" dia.) and a resistance of $0.415 \Omega / \mathrm{ft}$. As the current is rather high, a fast pulse of $1 \mathrm{~ms}$ is used to minimize coil heating as well as minimize dead-time between measurements. There was no measurable difference in the $0.1 \mathrm{mG}$ residual field inside the polarimeter's shielding before and after full strength coil pulses. To minimize the amount of systematic asymmetry introduced into the polarization measurement due to sample aging or light source intensity decay overtime, the coils are pulsed to flip the magnetization back and forth many times, accumulating data for each magnetization direction. Currently this is done about once per minute.

An above view of the target, analysis stage, and coil assembly inside the Faraday-cage drift space is shown in Figure 5.18(b). The insulated wires of the coils have been shielded by ribbons of thin sheet metal coated with colloidal graphite to ensure they do not cause any electrostatic beam deflections.

The target stage assembly rides on a high precision 5-axis manipulator, commercially purchased from Elmitec. The manipulator action can be seen in Figure 5.16, and it provides small alignment translations of the sample along the $x$ and $y$ axes (defined in the figure) and 2" of travel along z to allow moving the target assembly back and forth between the prep chamber transfer position and the analysis position in front of the detector. The manipulator also provides $\pm 2^{\circ}$ of target tilt rotation about both orthogonal in-plane axes. The entire manipulator is mounted on a large rotary feedthru, allowing the entire target assembly to rotate about the optical axis ( $z$ in the figure). As the polarimeter is sensitive to the polarization component of the incident beam along the target's magnetization axis, 
this rotation allows this sensitive axis to be freely adjusted to any orientation within the polarimeter's $x-y$ plane. The magnetizing coils are attached to the target stage so they rotate along with it. The rotational symmetry of the annular detector and conical grid are key to accommodating this freedom. This rotation allows the polarimeter to measure two orthogonal components of the incident polarization. Access to the third component requires rotation of the entire polarimeter with respect to the beam source, as discussed in section 4.5.1.

\subsection{Summary}

Although measurement of electron spin polarization is highly desirable for a wide range of experiments, it is currently not an easy task as polarimetry techniques must rely on inefficient spin-dependent scattering schemes. The most popular instruments utilizing spinorbit effects such as the state-of-the-art 'mini-Mott' polarimeters operate with efficiencies

of $\sim 1 \times 10^{-4}$ compared to simple intensity measurements. Utilizing exchange effects in low energy scattering from magnetic surfaces offers an attractive alternative with enhanced efficiencies, largely due to the much larger scattering cross sections. The custom exchange polarimeter developed here for the spin-TOF spectrometer promises a 10-100 times gain in efficiency over even the best Mott (or other spin-orbit based) polarimeters. 


\section{Chapter 6}

\section{SARPES with the spin-TOF spectrometer}

What a difference between a truth that is glimpsed and a truth that is demonstrated

- Unnamed, $19^{\text {th }}$ century French admirer of Newton

\subsection{The spectrometer}

Full assembly of the spin-TOF spectrometer has recently been completed allowing initial testing and usage. Key operational details of the full instrument are summarized. The spectrometer was installed with a small UHV photoemission endstation at beamline (BL) 12.0.1 at the ALS during 2-bunch operation and showed promising performance. Early results including successful spin- and angle-resolved photoemission spectroscopy will be briefly reviewed and the next steps will be identified.

\subsubsection{Combining the TOF lens system and exchange polarimeter}

The spin-TOF spectrometer takes a radical departure from the customary approaches to spin-resolved photoemission instrumentation by combining the spin analysis of the custom 


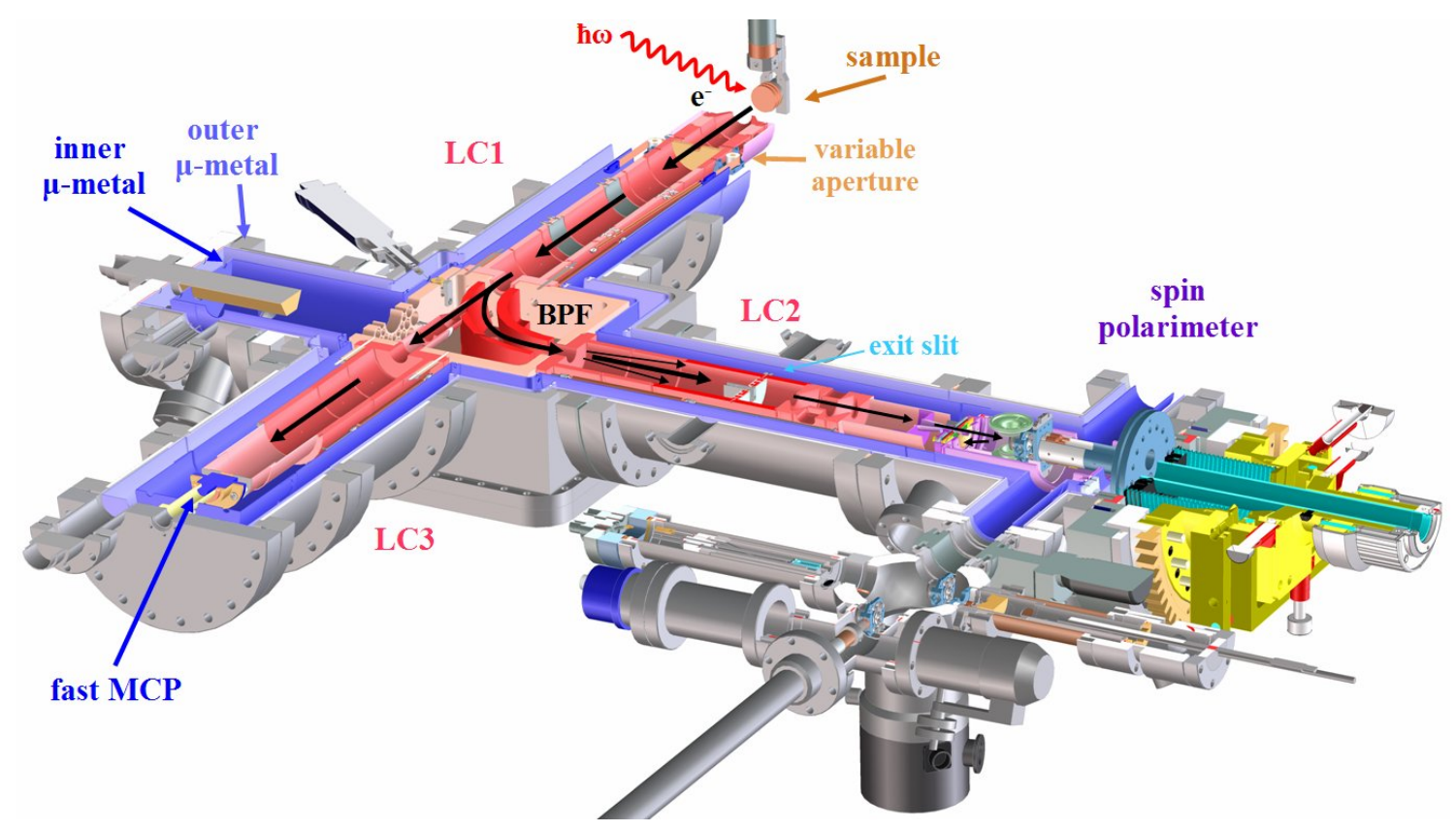

Figure 6.1. Cross sectional view of the complete spin-TOF spectrometer, to scale. Electron beam paths for both modes of operation are shown.

low energy exchange polarimeter of Chapter 5 with TOF energy analysis of the electrostatic lens system of Chapter 4. Although the two subsystems were previously discussed as independent instruments in separate chapters, the polarimeter is in fact seamlessly integrated into the TOF measurement by providing the electron arrival times with high time resolution. The full instrument is shown in cross section in Figure 6.1.

The design of the TOF lens system allows two modes of operation, represented by the two distinct electron paths illustrated by black arrows in Figure 6.1. In Mode \#1, the bandpass filter (BPF) formed by the $90^{\circ}$ spherical electrodes is turned 'off', allowing the electrons to travel a 'straight' path from Lens Column 1 (LC1) through to LC3. In Mode \#2, the BPF is turned 'on', deflecting electrons around the $90^{\circ}$ bend and down LC2. Although any electron detector can be installed at the end of either path, the primary setup will have the regular spin-integrating MCP detector assembly at the end of LC3 (Mode \#1) and the spin polarimeter at the end of LC2 (Mode \#2).

The two modes of operation provide valuable flexibility that allows the experimenter to take advantage of the particular strengths of both modes. Although the polarimeter 
involves large efficiency increases over other instruments, it is still less efficient than a spinintegrating detector. The use of Mode \#1 gives the ability to take extremely fast, full energy window photoemission spectra of the sample being probed. This can be used for quick and accurate sample surface characterization, through either the sharpness of near $\mathrm{E}_{F}$ dispersing valence bands, or through the core level peaks which are automatically included in each spectrum. Charactering the band dispersions can also be used for alignment of the sample's crystallographic axes. This fast-acquisition mode can also be used to search for ideal photon energies, or for areas within momentum-space and binding energy where spin-resolution is most desired. Any of these activities would be more difficult and time consuming if performed with the spin polarimeter.

Once the sample is aligned and points in $k$-space and windows in binding energy are identified where spin-resolution is most important, the experimenter can switch to Mode \#2, sending the photoelectrons around to LC2. Photoelectrons within the defined energy window pass through the exit slit and are focused into the spin polarimeter; spin-resolved data is obtained through the difference spectrum between when the polarimeter target is magnetized 'up' and 'down', as detailed in Chapter 5. The combined improvements of the new polarimeter and the parallel energy detection of the TOF technique allow these spinresolved spectra to be taken faster and therefore with higher resolutions than are possible with other existing instrumentation.

With the full picture of the spin-TOF instrument in mind, it can be seen that the $90^{\circ}$ BPF performs three important functions. First, the BPF acts as a switchyard between LC2 and LC3, allowing instantaneous switching between two different detectors. Second, it removes the unwanted parts of the spectrum which would cause spectrum 'overlap' during operation at high energy resolution (larger retardation / lower average velocity), as detailed in section 4.4.5. Thus we see that Mode \#2 allows higher energy resolution than Mode \#1. Third, placing the polarimeter after a $90^{\circ}$ bend allows changing the orientation of the polarimeter target with respect to the photoemission sample, allowing polarization measurement along all three orthogonal directions (see section 4.5.1).

Chapter 5 spent much time focusing on the efficiency of spin polarimeters described 
by their Figures of Merit (FOMs). As discussed in refs 170,107, a FOM for an entire spectrometer must also take into account the loss of any electrons between the energy analyzer and the spin polarimeter. The possibility of a loss of electrons results from general conservation laws of electron optical phase space. The law of Helmholtz-Lagrange requires that the electron optical phase space of an electron beam, defined as

$$
\Phi=E A \Omega
$$

is a conserved quantity at all points along a paraxial electron optical system. ${ }^{158}$ In the above, $E$ is the beam kinetic energy, $A$ is the beam cross sectional area, and $\Omega$ is the beam's solid angle. If the polarimeter can accept a given electron optical phase volume, $\Phi_{\text {pol }}$, which is smaller than the occupied electron optical phase volume of the upstream electron optics (i.e. an energy analyzer), then electrons must be lost and total efficiency is reduced before even considering the FOM of the polarimeter.

The current spin polarimeter operates at very low scattering energies from around 2 to $10 \mathrm{eV}$ and has severe geometric restrictions on the accepted electron beam which must pass through the $6 \mathrm{~mm}$ diameter tube through the annular MCP (see section 5.4.2). The TOF lens system must retard the photoelectron beam to be analyzed to an energy of 2-10 eV as well as focus it through the MCP tube and onto a small spot on the scattering target. From the tube's geometry, we can define that a beam with an angular divergence of $1^{\circ}$ half angle focusing to a $1 \mathrm{~mm}$ spot on the scattering target can be accepted. This translates to an electron optical phase space acceptance of $0.0015<\Phi_{\text {pol }}<0.0075$ in units of $\mathrm{mm}^{2} \mathrm{sr} \mathrm{eV}$. This acceptance is quite small compared to values of $10^{3}$ for 'mini-Motts' (and small even compared to 1 for spin-orbit LEED polarimeters). ${ }^{158}$ The small acceptance is due mostly to the much lower scattering energy.

This value for $\Phi_{\text {pol }}$ is not a cause of large concern for the present application to ARPES, however. Although the acceptance of the TOF lens system (and other energy analyzers) could be much larger, the phase space acceptance is in fact purposely reduced to achieve good angular resolution in the photoemission measurement. The angular resolution of the spin-TOF spectrometer is defined simply by its angular acceptance; the variable aperture at the front of $\mathrm{LC} 1$ can reduce this to as low as around $\pm 1^{\circ}$. Additionally, the synchrotron 


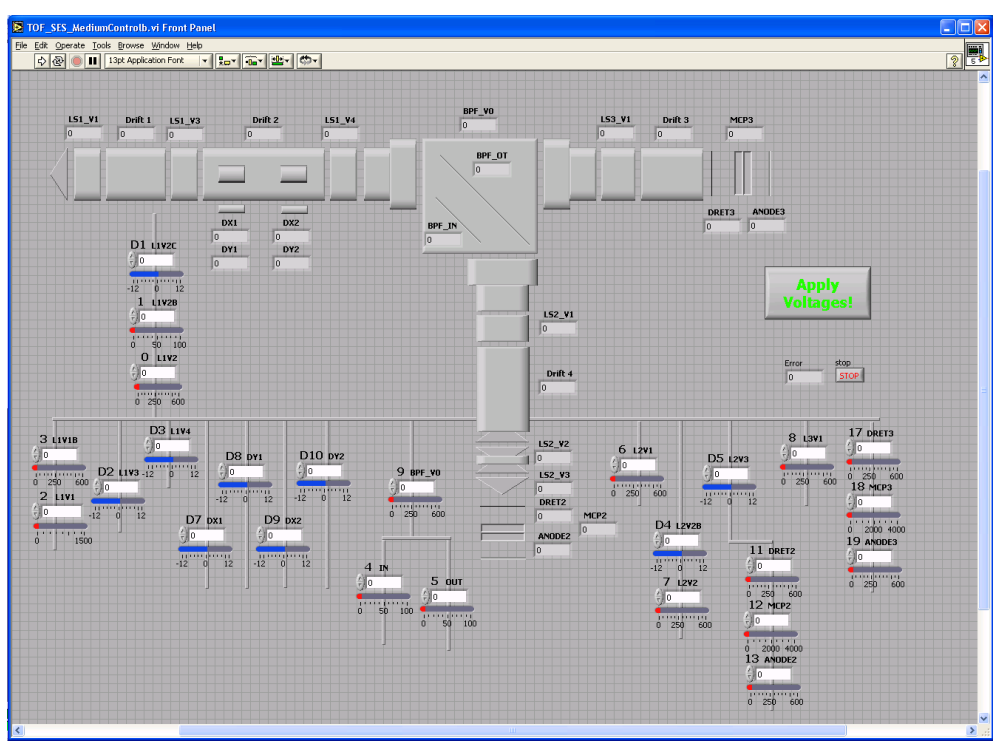

Figure 6.2. Screenshot of power supply control software.

and laser based light sources the spin-TOF is meant to be used with can achieve small spot sizes of $<100 \mu \mathrm{m}$ quite easily. At initial photoemitted kinetic energies of $100 \mathrm{eV}$, this means the electron optical phase space volume of the beam allowed to pass through the analyzer will only be $\Phi_{\text {beam }}=0.0008 \mathrm{~mm}^{2} \mathrm{sr} \mathrm{eV}$ anyway. Thus, with ideal operation of the TOF lens system, there should be no fundamentally required loss of electrons accepted into the spin polarimeter.

\subsubsection{Instrumental control}

All spin-TOF electrode voltages are supplied by the flexible power supply system used by the popular VG Scienta hemispherical analyzers. The system consists of a rack-mounted cabinet with an array of individual, swappable, floating power supply boards, each one digitally controlled via fiber-optic link to a central computer interface board. Computer communication is provided by a USB interface. The Scienta power supply system was chosen due to the number of systems already in use on the ALS floor; possible replacement supply boards abound in case of any failures.

The power supply computer interface is supplied with a complete set of DLLs that made 
building custom control software straightforward. A screenshot of the 'LabVIEW' based software panel for setting the 21 independent voltages via 25 individual supplies is shown in Figure 6.2. The panel shown allows direct control of each supply voltage with a display of the resulting voltages on each electrode. Additional panels allow automatically loading predefined sets of electrode voltages, or 'lens tables'.

Power from each supply is taken through individual durable high-voltage cabling to Junction Boxes (JBs) at each vacuum electrical feedthru directly on the spin-TOF vacuum chamber. The JBs contain low-pass RC circuitry to remove noise from the switching power supplies as well as the necessary wiring for 'stacking' the voltages of certain power supplies before connection to a feedthru pin. Each pin is then directly connected to its corresponding electrode(s) within the spin-TOF spectrometer. A wiring diagram, showing the power supply connections within each JB, each pin-electrode connection, and each external JB-JB SHV coaxial connection in shown in Figure 6.3. The spin polarimeter's target magnetization coils are powered by a National Instruments 'BNC-2110 Shielded Connector Block' connected to computer through a National Instruments 'PCI-6221 M Series Multifunction DAQ' card. Pulses are shaped and controlled through a separate LabVIEW control panel. The resulting output of the BNC-2110 is first amplified by a custom high-speed inverting $50 \mathrm{~V}$ amplifier, and then supplied directly to the coils via electrical feedthrus installed in the target manipulator.

As described in section 4.4.2 and 4.4.4, data acquisition is performed by the computer controlled Ortec 9308 pTA. A simple LabVIEW based control panel was also written to interface with the pTA's own set of DLLs. The software panel currently provides control of the pTA along with multiple displays for viewing the incoming data with respect to time as well as kinetic energy and for live comparisons with recent spectra. The panel saves data in the FITS file format for flexible organization of various amounts of 'meta-data' such as pTA and/or power supply settings used during acquisition. 


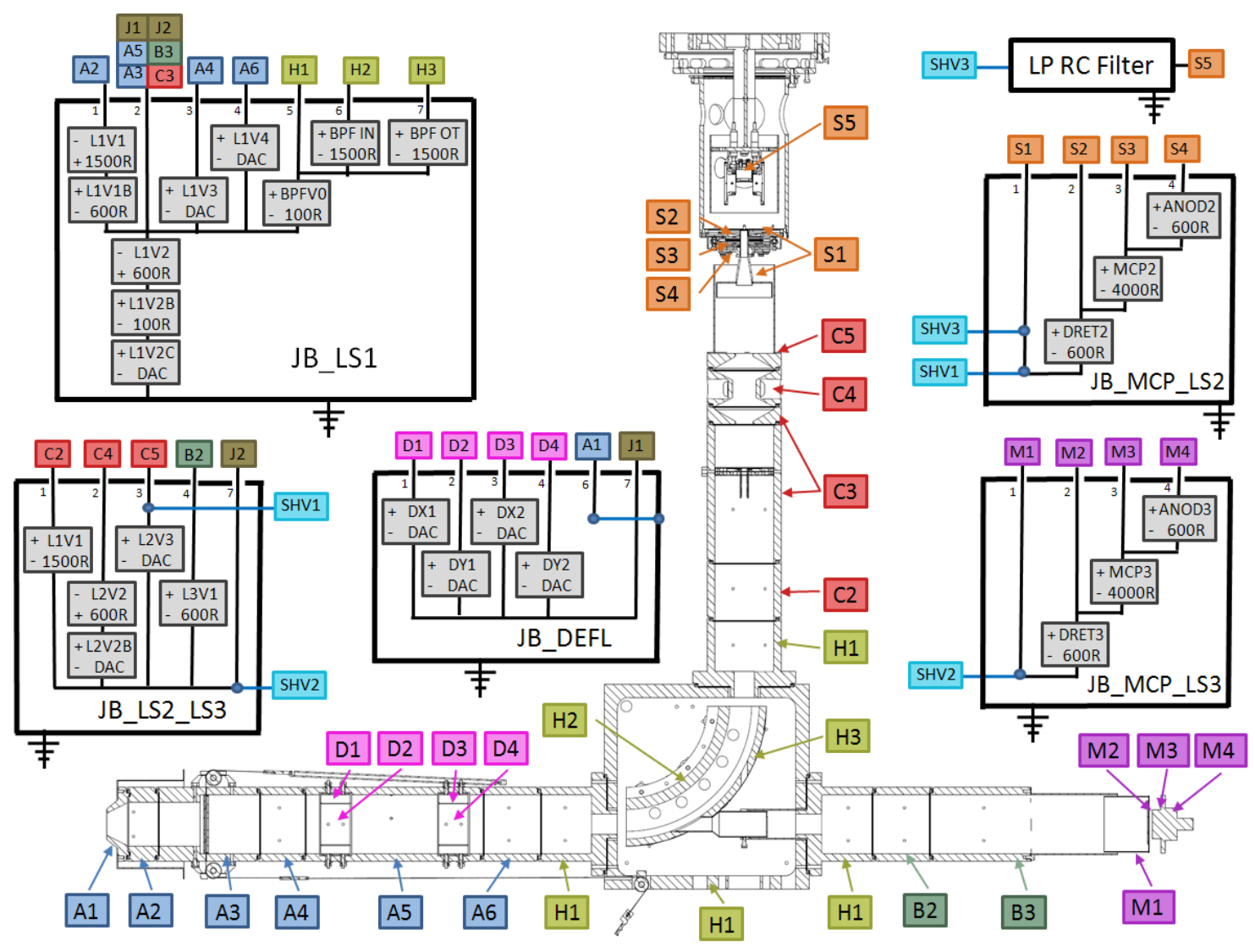

Figure 6.3. Diagram of spin-TOF electrical wiring connections. 


\subsubsection{Experimental endstation}

The spin-TOF is installed on a very basic UHV chamber system for initial testing and usage. The analysis chamber itself is based on a 6-way 8" CFF cross mounted on a ' $80 / 20$ ' aluminum extrusion modular support frame. Pumping is supplied by a $300 \mathrm{~L} / \mathrm{s}$ Varian V300 turbopump, a 300 L/s Varian Diode ion pump, and a 6" ALS-style titanium sublimation pump (TSP) can, all mounted from below on a pumping cross. The analysis chamber is equipped with a double layer of magnetic shielding which directly couples to the shielding of the spin-TOF spectrometer.

The sample stage contains an e-beam heater assembly compatible with ALS-style 1" sample pucks, directly mounted to a 1" diameter cryostat tube. Thus the sample stage provides high temperature sample heating, as well as cooling to near liquid helium temperatures, but does not provide any motion apart from motion of the entire cryostat. The cryostat is vertically mounted on the analysis chamber through a VG 'Centiax' large-bore XYZ manipulator (providing three translational degree of freedom to the cryostat/sample) and a small 4.5" CFF differentially pumped rotary seal (providing rotation of the cryostat/sample about the cryostat's vertical axis).

The sample can be vertically raised out of the analysis chamber into a small 'prep' chamber (formed by a 6-way 4.5" CFF cross) which features an externally mounted solenoid shaped coil for magnetizing the sample vertically. The 'prep' chamber also has room for evaporators for sample growth in addition to the flanges directly on the analysis chamber available for evaporators. The analysis chamber location provides the ability to evaporate on the sample during simultaneous photoemission measurement. A photograph of the spinTOF spectrometer and test endstation installed at the ALS BL 12.0.1 during the August 2008 2-bunch mode is shown in Figure 6.4.

\subsubsection{Bandpass filter details}

Mode \#1 of the spin-TOF operates very similarly to the prototype lens system as described in section 4.4.4. The BPF of Mode \#2, however, introduces additional complexities. 


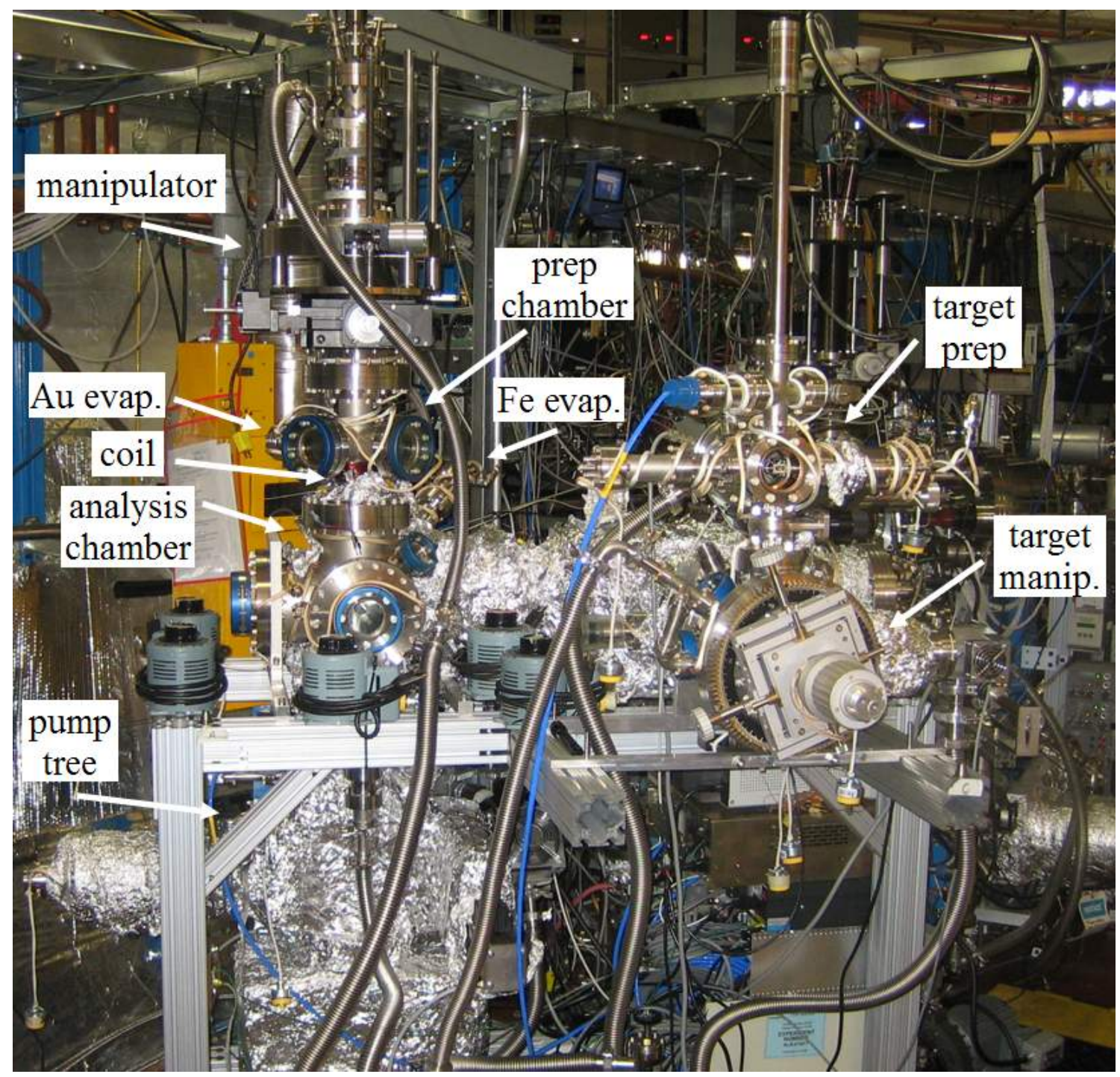

Figure 6.4. Spin-TOF spectrometer and test endstation at BL 12.0.1. The analysis chamber is on the left, and the spin-TOF extends towards the right. The spin-TOF Lens System 2 is angled towards the camera affording a good view of the rear of the spin polarimeter, its target manipulator, and its target prep chamber extending above it. 
One particular problem is the introduction of timing aberrations due to path length variations caused by the BPF. That the BPF may introduce path length variations can be qualitatively understood by the fact that this is what makes the BPF (and of course SDAs) work: electrons of different energies take different paths around the spherical electrodes. Although timing dispersions introduced by the BPF is an important problem, is was decided early in the project that they could be sufficiently minimized with correct lens tables such that they did not limit the final energy resolution.

The issue has been only briefly experimentally investigated at this point. To work on this aspect as well as others, a photocathode electron gun was developed. Using $4.5 \mathrm{eV}$ light (from third harmonic generation) of the lab's mode-locked laser, semi-monochromatic electron pulses of initially 1 ps width are generated from a polycrystalline gold surface floating at an arbitrary potential which directly controls the kinetic energy of the electron pulses. These pulses were first measured with the spin-integrating detector in Mode \#1, and showed a $89 \mathrm{meV}$ pulse width. The same electron pulses were then measured in the polarimeter using the $\mathrm{BPF}$ in Mode \#2 where they showed a $155 \mathrm{meV}$ pulse width. The two measurements are compared in Figure 6.5(a). These results show that the lens table used for the Mode \#2 measurement likely leads to a large amount of timing aberrations which have a significant impact on the resolution, limiting performance to $155 \mathrm{meV}$ energy resolution. Due to time constraints prior to the scheduled ALS 2-bunch runs, lens table adjustment was not possible before taking the data shown in the rest of the Chapter. An important next step of spectrometer development will be tuning the lens tables to bring these timing dispersions down to acceptable levels for sub-10 meV resolution. It may be additionally possible that timing aberrations are introduced within the spin-polarimeter itself; it will be important to investigate the timing properties of the polarimeter and the BPF independently. This can be done by switching the locations of the polarimeter and regular detector behind LC2 and LC3.

The BPF adds another complexity, as well. While Mode \#1 acquires an entire spectrum simultaneously, Mode \#2 acquires a spectrum only within a window (usually on the order of $1 \mathrm{eV})$. Although the transmission percentage of electrons through the lens system in 

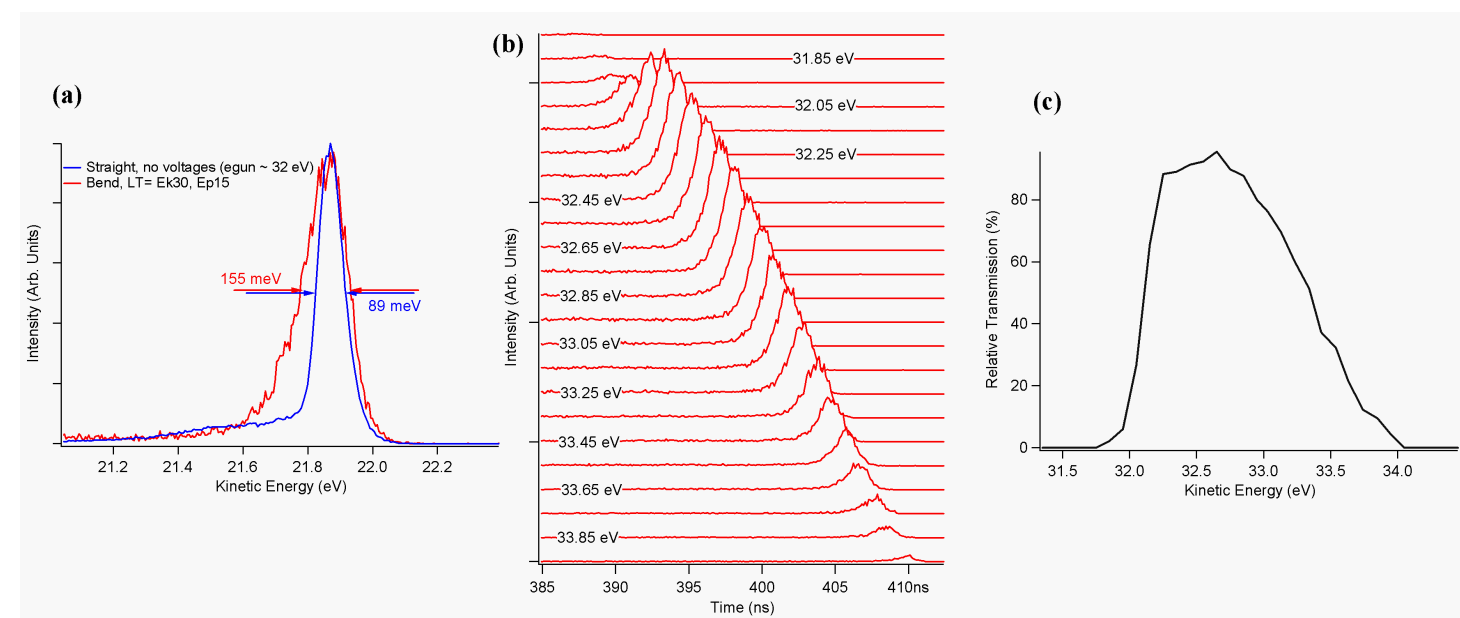

Figure 6.5. Properties of Mode \#2 with BPF and polarimeter tested with photocathode electron source. (a) Comparison of electron source spectral width measured with Mode \#1 (no lens voltages) and Mode \#2. (b) Scanning kinetic energy of electron pulses through the bandpass window of Mode \#2. (c) Measured transmission function extracted from peak heights in (b).

Mode \#1 may be somewhat kinetic energy dependent, the resulting 'transmission function' will not be so strong as to mask features of the real spectrum (as evidenced by the spectra taken from the prototype system in section 4.4.4 and the spectra below in Figure 6.6). The transmission function of Mode \#2 and the BPF must be a strong function of energy by definition, as it needs to be high for energies within the bandpass window, and extremely low for energies outside this window. Ideally the transmission function would be a perfect 'square' shape, with $0 \%$ transmission everywhere except for a sharply defined window of $100 \%$ transmission. This is not completely achieved, and one must correctly deal with the influence of the real transmission function on the spectral lineshape.

One approach is to directly measure the transmission function. This can allow 'tuning' of the lens tables and exit slit size to find transmission which more closely approximates the ideal square transmission, as well as a quantitative way to interpret future spectra. This has also only just begun using the same electron source. The electron pulses were again measured in Mode \#2 as in Figure 6.5(a), for a large set of cathode potentials. Each resulting spectrum is shown in Figure 6.5(b) with a vertical offset for clarity. As the kinetic energy of the electron pulse 'scans' through the bandpass window, the measured intensity 
amplitude rises and falls, showing the effective transmission function of the lens table. Figure $6.5(\mathrm{c})$ is a plot of the pulse intensity amplitude as a function of cathode potential, which is at least approximately related to the actual transmission function. Further efforts with tuning may be able to provide a more 'square' shape. In the end, it may be most convenient to acquire data in a 'swept mode' where the BPF voltages are scanned to sweep the bandpass transmission function across the window of interest, thus integrating out the transmission function. This technique will also allow taking continuous data through an energy window larger than that allowed by a fixed bandpass.

At the time of the ALS 2-bunch run, only the one lens table used for the data in this section was available. Although several steps of testing and calibration still need to be performed for the BPF and Mode \#2 to be fully operational, we will see in the next section that qualitatively reasonable spectra can already be obtained with Mode \#2 with the current lens table. By correct selection of the photon energy, the desired part of a spectrum can be placed within the kinetic energy range of the bandpass given in Figure 6.5(c). This has allowed spin resolution with the polarimeter to be clearly demonstrated.

\section{$6.2 \quad$ Spin-resolved ARPES}

The 10 days of the August 2008 ALS 2-bunch mode period presented an opportunity to demonstrate the current capabilities of the spin-TOF spectrometer. As noted, work remains for full calibration of the instrument, particularly in regards to development of further lens tables and a more extensive software control package. Utilizing the 2-bunch beamtime to investigate its present performance, however, is quite valuable for making great progress in these final developmental steps.

\subsubsection{Sample preparation}

For this beamtime, the test chamber system was equipped to investigate two sample systems with spin-dependent bandstructures: Fe(110) and $\mathrm{Au}(111)$ surfaces. These systems were chosen as they are prototypical examples of spin-dependent electronic structures which 

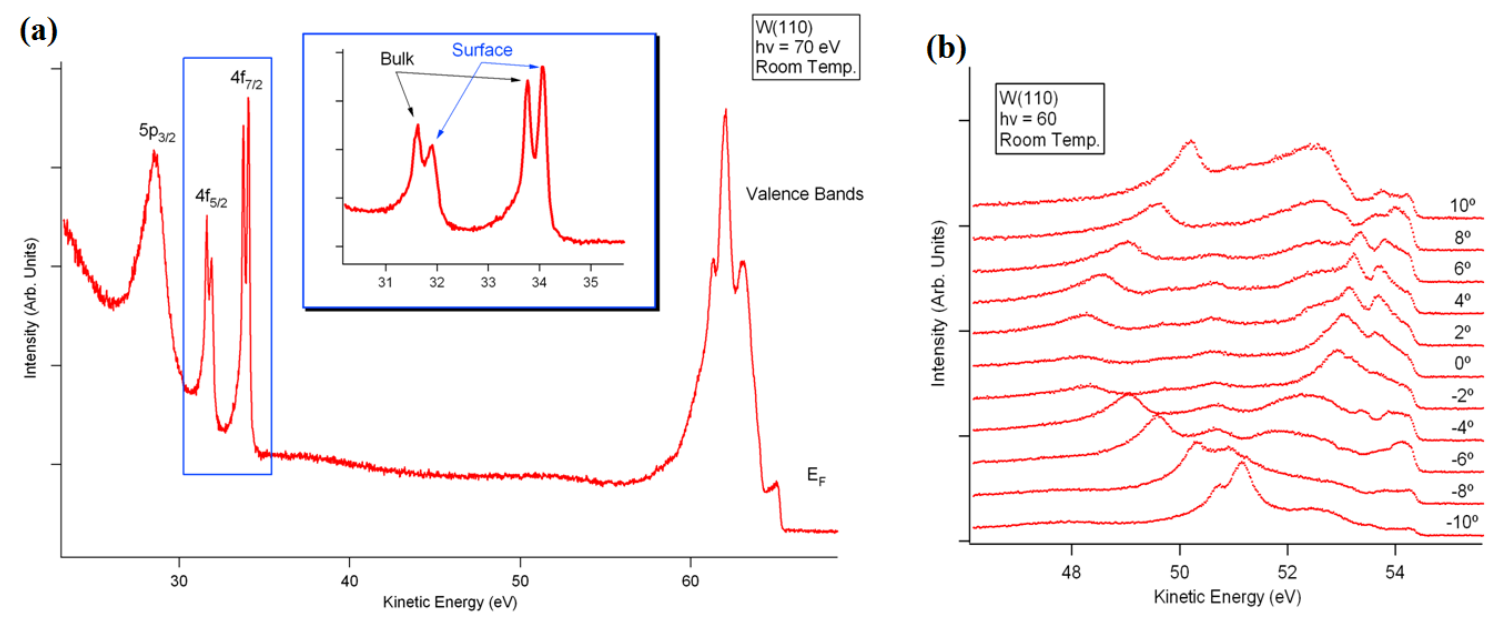

Figure 6.6. Spin-integrated photoemission spectra from a clean W surface at room temperature with $70 \mathrm{eV}$ photons taken with the spin-TOF Mode \#1. (a) A full range spectrum including several core levels. Inset is a close-up view of the $4 \mathrm{f}$ core levels, corresponding to the blue box. (b) Spectra taken with $\mathrm{h} \nu=60 \mathrm{eV}$, shown only in the valence band region, at several emission angles, revealing dispersive bands.

have been previously studied by spin-resolved photoemission. Additionally, clean surfaces of both can be conveniently obtained by in-situ evaporation of epitaxial thin-films on a $\mathrm{W}(110)$ substrate, which in turn is easily prepared by the usual recipe (see section 5.4.1). Thus, a high quality W(110) crystal (10 mm diameter by $1.5 \mathrm{~mm}$ thick) was installed on the endstation's cryostat/heater sample stage, an e-beam Fe evaporator was mounted on the analysis chamber, and a simple resistive Au evaporator was mounted in the endstation's prep chamber. Spin-TOF results from these systems are presented in the following sections.

As both systems required a multi-step preparation process, utilizing the spectrometer's Mode \#1 for high-speed sample characterization was extremely useful. Both systems required a very clean W(110) surface. The W surface can be straightforwardly cleaned with cycles of annealing and $\sim 2000^{\circ} \mathrm{C}$ flashing in a partial pressure of $\mathrm{O}_{2}$, but how clean the surface becomes should somehow be verified. A spin-integrated spectrum taken in Mode \#1 after a successful $\mathrm{W}$ cleaning procedure is shown in Figure 6.6(a). The spectrum shows a sharp Fermi edge and strong valence band peaks. It also shows a distinct set of core level peaks corresponding to the $5 \mathrm{p}_{3 / 2}, 4 \mathrm{f}_{5 / 2}$, and $4 \mathrm{f}_{7 / 2}$ levels. First, the locations of these peaks identifies that the illuminated spot of the sample is indeed W. This in itself is useful; when 
the sample was incorrectly position such that part of the Ta pieces holding the $\mathrm{W}$ crystal in place was illuminated instead, these core levels shifted to the corresponding Ta energies, easily identifying the sample mis-positioning.

Furthermore, taking a close look at the $4 \mathrm{f}$ core levels (figure inset) shows that each $4 \mathrm{f}$ level is further split into two peaks. The higher kinetic energy (lower binding energy) set corresponds to surface core levels, shifted from the bulk contribution due to changes in the surface density of states and other effects of the reduced coordination at the surface. ${ }^{180,181}$ Observing such a strong surface contribution peak suggests a quite clean surface; indeed the data agrees remarkably well with that found in the literature ${ }^{182}$ in terms of peak separations, heights, and widths. In contrast, it was quickly found that a dirty surface from too much time exposed to the residual gases in the vacuum or from an insufficient cleaning procedure would result in reduced heights (or complete lack) of the surface peaks. Thus, the fast, full spectrum capabilities of Mode \#1 are quite useful for sample characterization and preparation.

Figure 6.6(b) shows expanded views of the valence band portions of spectra at different emission angles along the $[0,0, \overline{1}]$ direction. The observed dispersing bands demonstrate the ARPES abilities of Mode \#1. These dispersions can be used for verifying sample quality, and their symmetry can be used for alignment of the sample's crystallographic axes as well as identifying locations in $k$-space.

Mode \#1 was also quite useful for monitoring the growths of both sample systems. High quality Fe(110) films can be epitaxially grown on clean W(110) substrates as widely noted in the literature. ${ }^{175,176,177}$ The magnetization 'easy-axis' is found to be along the $[1, \overline{1}, 0]$ direction for thin films under $60 \AA$ but then flips to the bulk $[1,0,0]$ direction for thicker films. The spin-resolved experiment becomes much more straightforward when the easy-axis is known, and so the thickness, and therefore the evaporation rate, needs to be fairly well known. With the Fe evaporator mounted on the analysis chamber, fast Mode \#1 spectra could be taken during Fe film growth. Monitoring the disappearance of W features (core levels and valence lineshapes) from the spectra allowed a good estimate of the evaporation rate. For instance, as the mean free path of photoelectrons are $\sim 5 \AA$ at 
$\sim 30 \mathrm{eV},{ }^{183}$ approximately $5 \AA$ had grown when the $\mathrm{W}$ 4f core levels decreased in intensity by about a factor of 1/e. Evaporation was then continued for about 5 times longer for a $\sim 25 \AA$ Fe film.

As the only room for a $\mathrm{Au}$ evaporator was above the analysis position in the prep chamber, Au films were grown on the W(110) substrate without the aid of live photoemission monitoring. However, the spin-dependence of the Au system does not have the thickness dependence that the Fe system does, and so thickness calibration is not as important as

long as more than a few monolayers are deposited. Evaporation proceeded by heating a small lump of $\mathrm{Au}$ in a tungsten wire basket until it became molten, and leaving the $\mathrm{W}(110)$ substrate in front of it for $\sim 12$ minutes. The resulting film was not thick enough to be visible by eye, but when the sample was returned to the analysis position and measured with Mode \#1, it displayed an obvious Au spectrum (just like those taken from Au in section 4.4.4) with no trace of $\mathrm{W}$ core levels. Additionally, a clear peak near $\mathrm{E}_{F}$ due to the $\mathrm{Au}(111) L$-gap Shockley surface state was present, signaling that a quality sample for further spin analysis had been obtained.

In all of the above, Mode \#1 was utilized with each electrode of LC1 and LC3 grounded. In other words, no retardation or focusing was used, or found necessary for the present uses. Indeed, these cases illustrate the full spectrum capabilities of Mode \#1 as a large strength; any retardation reduces the total energy width of the spectrum and one perhaps misses out on helpful high binding energy core levels. The energy resolution obtained without any retardation was also clearly sufficient for the present uses of merely identifying key features such as the Fermi edge and valence and core peaks. Lens tables for retarding, focusing, and increasing count rates will be developed, however the count rate was already quite sufficient with the flux available in these current experiments.

\subsection{2 $\mathrm{Fe}(110)$}

The $3 \mathrm{~d}$ ferromagnetism of bulk Fe makes it an ideal system to study with spin-resolved techniques, and it has historically been a popular choice for spin-resolved photoemis- 


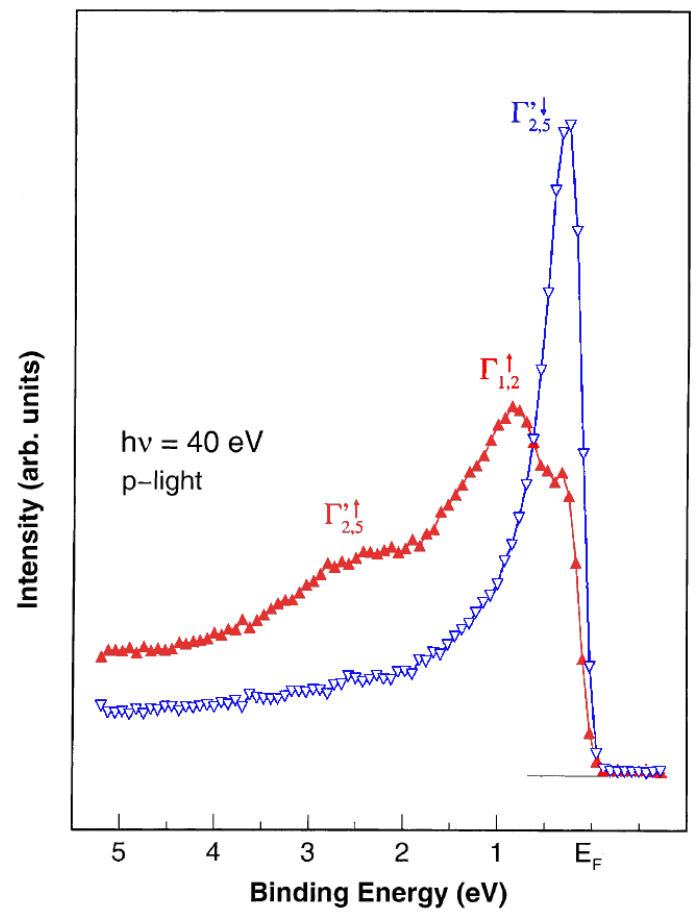

Figure 6.7. Spin- and angle-resolved photoemission spectroscopy of Fe(110). The data has been decomposed into minority (blue) and majority (red) spectra. Figure adapted from ref. 184.

sion. ${ }^{100,22,24}$ As discussed in section 3.3, spin-resolved photoemission can measure the exchange splitting of the minority and majority bands and directly confirm the spin character of each. As Fe has the largest exchange splitting of the $3 \mathrm{~d}$ ferromagnets, it is an ideal system for giving the spin-TOF a 'test-drive'. An example of previous SARPES data taken in normal emission from $\mathrm{Fe}(110)^{184}$ is shown in Figure 6.7. The data was taken with 40 $\mathrm{eV}$ photons with $100 \mathrm{meV}$ and $2^{\circ}$ energy and angular resolutions, respectively, and shows a strong minority peak near $\mathrm{E}_{F}$ and a majority peak at about $0.6 \mathrm{eV}$ higher binding energy. Note that this data is quite similar to the data taken from the Fe(100) face, presented earlier in Figure 3.3(a). The large difference between the majority and minority emission near $\mathrm{E}_{F}$ means that Fermi level photoelectrons have a large, and therefore 'easy' to measure, polarization, $P$, making it a good system to start with.

The $\mathrm{Fe} / \mathrm{W}(110)$ sample was first prepared as in section 6.2.1. From the recipes in the literature, ${ }^{175,176,177,184}$ the best results are obtained when the growth is either performed with the substrate held at an elevated temperature of $~ 500 \mathrm{~K}$ or performed at room tem- 


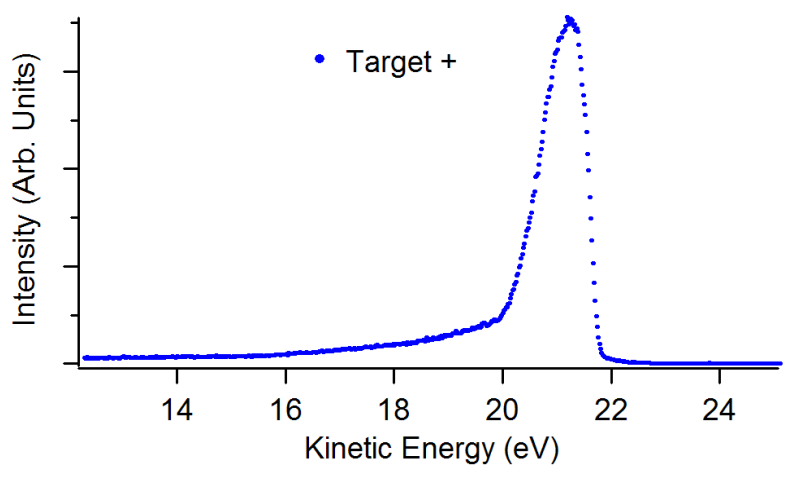

Figure 6.8. Mode \#2 spectrum of $\mathrm{Fe} / \mathrm{W}(110)$ system with $\mathrm{h} \nu=36.6 \mathrm{eV}$ and the polarimeter target magnetized by a positive coil pulse.

perature followed by a brief anneal to $\sim 500 \mathrm{~K}$. As the current setup does not include the means for measuring this range of temperatures, the growth was performed at room temperature followed by a quick anneal of an elevated temperature that was not enough to visibly glow (i.e. $<600^{\circ} \mathrm{C}$ ). Without a LEED, it was difficult to tell if the anneal was enough to obtain perfect crystallinity, although even a polycrystalline Fe film is expected to give spin polarized photoemission. ${ }^{185}$ The sample was raised to the prep chamber coil position and magnetized upwards with a current which prior to the experiment resulted in a measured field of $230 \mathrm{G}$. The sample was then returned to the analysis position in front of the spectrometer.

The BPF was turned 'on' and spectra were collected in Mode \#2 with the spin polarimeter. As the only available lens table at the time set the bandpass window for photoelectrons of an initial kinetic energy of $\sim 32.5 \mathrm{eV}$ (Figure $6.5(\mathrm{c})$ ), the photon energy was set to 36.6 $\mathrm{eV}$ (the difference being due to the work function). This was found to put the Fermi edge within the bandpass window while including as much of the rest of the spectrum as possible. A spectrum collected with the polarimeter target magnetized in a single direction is shown in Figure 6.8. It must be remembered that the lineshape shown is largely due to the BPF transmission function discussed above in section 6.1.4, although the sharp drop on the high kinetic energy side is also due to the Fermi edge.

Spin resolution is obtained by comparing such spectra with the polarimeter target magnetized in opposite directions. As the photoelectrons are expected to be polarized along 
(a)

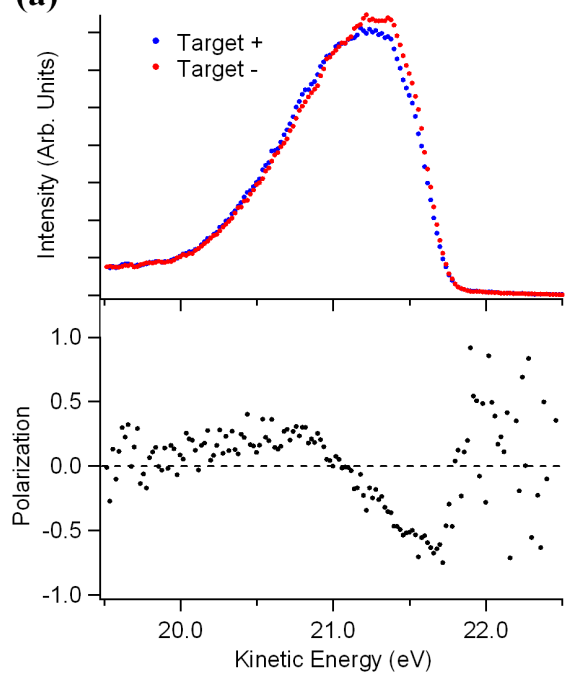

(b)

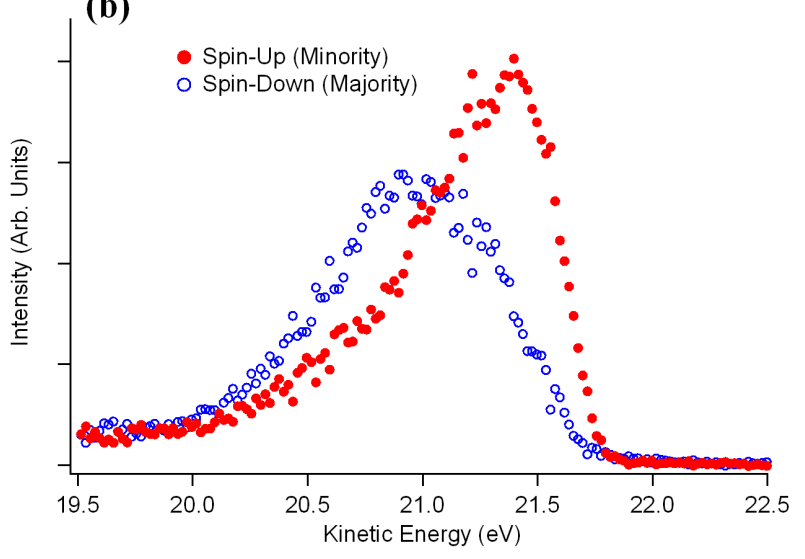

Figure 6.9. Spin-resolved spectra of Fe/W(110) with $\mathrm{h} \nu=36.6 \mathrm{eV}$. (a) Spectrum with polarimeter target magnetized by a positive coil pulse, same as Figure 6.8 with an expanded energy scale, in blue, and with polarimeter target magnetized by a negative coil pulse in red. The normalized difference, divided by the effective Sherman function, is the measured polarization, plotted below. (b) Majority and minority spectra derived from (a).

the sample's magnetization axis (vertical axis in current geometry), the polarimeter target is rotated such that it's magnetization direction is also vertical. Spectra were collected by reversing the target magnetization with positive and negative coil pulses at intervals of one minute. Spectra with the target magnetized by a positive coil pulse were added to form a ' + ' spectrum and the others added to form a '-' spectrum. Initial work using a target prepared as a $5 \mathrm{ML}$ Co film as discussed in section 5.4.1 lead to nearly identical '+' and '-' spectra with almost no asymmetry. However, when a $50 \mathrm{ML}$ Co target was prepared followed by a brief anneal $\left(<600^{\circ} \mathrm{C}\right)$, an obvious difference between the '+' and '-' spectra emerged, shown in the top panel of Figure 6.9(a). Perhaps the ultra-thin Co target did not perform well due to imperfect growth conditions which the thicker films are less sensitive to. Further work can be done to improve the target preparation recipes in order to obtain the high performance of the ultra-thin films, but the present thicker films have proven quite capable.

The spin polarization of the spectra is extracted as the difference between the ' + ' and '-' spectra, divided by the sum and divided by the effective Sherman function. From 
previous work with similar targets in the SPLEEM (see section 5.4.1), an effective Sherman function of $10 \%$ is reasonable. Experimentally, using this value gives the large polarizations expected from the Fe sample, but using lower values gives nonsense polarizations above 100\%. Although the Sherman function may have kinetic energy dependence, for now we will assume it to be small, and use the constant value of $10 \%$. The polarization calculated in this way is displayed in the lower panel of Figure 6.9(a).

The '+' and '-' spectra can then be decomposed into the spin-resolved majority and minority spectra shown in Figure 6.9(b) following equations 5.20 and 5.21. Note that the magnetic moment of the electron is opposite its spin direction, so although the Fe is magnetized upwards, the majority electrons are spin-down. The data shows that there is a strong minority spin peak right at $E_{F}$ causing a large negative spin polarization near the Fermi level. This reverses at kinetic energies about $500 \mathrm{meV}$ less than the peak in the minority spectrum, when a peak in the majority spectrum grows stronger. Further behavior at lower kinetic energies is disrupted by the bandpass cutoff. This behavior matches exactly that of the previous work ${ }^{175,184}$ shown in Figure 6.7, giving strong confidence that the spinTOF spectrometer is functioning correctly. Additionally, the agreement suggests that a quality, single-crystal Fe(110) surface was, in fact, obtained. Unfortunately, the limited remaining beamtime did not allow for taking a complete data set at multiple emission angles to observe spin-resolved dispersions.

To ensure that the asymmetry in the '+' and '-' spectra are indeed due to photoelectron spin polarization, the $\mathrm{Fe} /(\mathrm{W} 110)$ sample was magnetized downwards and the experiment was repeated. The asymmetry in the following '+' and '-' spectra switched, exactly as expected as the photoelectron spin polarization flipped direction. The extracted majority and minority spectra taken from the Fe sample magnetized down and up are compared in Figure 6.10(a). In the figure, the terms 'Spin-Up' and 'Spin-Down' are referenced to the fixed vertical axis of the laboratory, while the terms 'majority' and 'minority' reference to the Fe carriers which flip directions when its magnetization flips. The figure strongly confirms the interpretation of the spectral asymmetry as due to photoelectron polarization.

As a further check that our interpretation makes sense, the bandpass window was ef- 
(a)

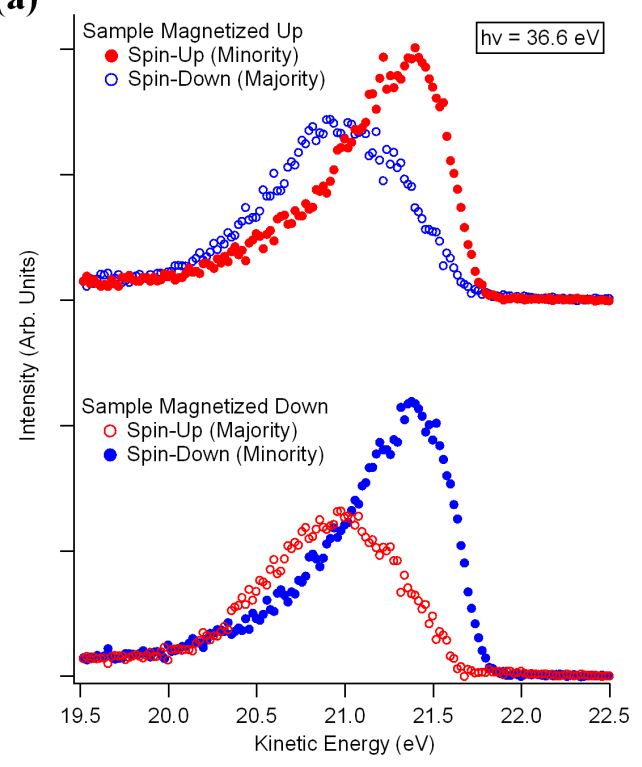

(b)

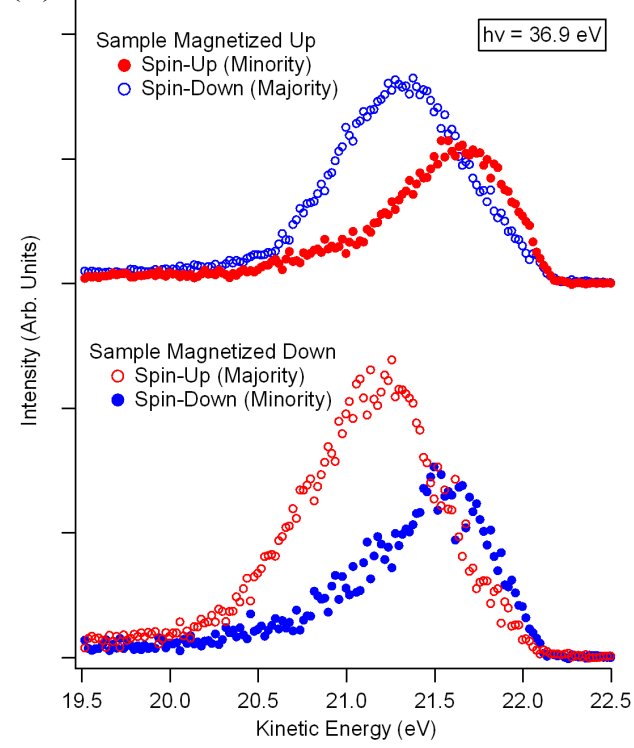

Figure 6.10. Spin-resolved spectra of Fe/W(110). Upper panels are with sample magnetized upwards, and lower panels are with the sample magnetized downwards. Spectra are taken with $\mathrm{h} \nu=36.6 \mathrm{eV}$ in (a) and $\mathrm{h} \nu=36.9 \mathrm{eV}$ in (b).

fectively moved to larger binding energy by increasing the photon energy by $0.3 \mathrm{eV}$. The resulting spin-resolved spectra, again taken with the Fe sample magnetized both up and down, are shown in Figure 6.10(b) to be directly compared with panel (a). Here, the sharp edge on the high kinetic energy side of the minority spectra are distorted as the Fermi edge is cut-off by the transmission function. As the minority peaks are depressed by the high energy side of the transmission function, the majority peak becomes stronger as it moves into the peak of the transmission function. This, again, is fully consistent with the picture of the full spin-resolved spectra of Figure 6.7. With completed lens tables, the spectrum could be taken across an arbitrarily large energy window by successively scanning the bandpass window with the correct voltages applied to the BPF electrodes. As is, the present data presents strong proof that the spin-TOF spectrometer is capable of spin-resolved photoemission spectroscopy.

Each spin-resolved set of spectra shown took $\leq 30$ minutes for the spin-TOF to acquire; this is likely significantly less than the previous work required for the data in Figure 6.7. Additionally, careful comparison shows that the present data contains more than 3 times 
more data points, and the TOF-limited energy resolution should be 5 times better than the $100 \mathrm{meV}$ resolution the Figure 6.7 data was taken with. The present spin-TOF data was also taken using the large $3 \mathrm{~mm}$ beamspot described in section 4.4.4 and shown in Figure 4.9. The lens system is meant for transmission of photoelectrons from a $100 \mu \mathrm{m}$ beamspot, and so many photons are effectively wasted when spread through such a large spot. Total count rate and efficiency will be much improved with a reduced beamspot. Although the present spin-TOF data is slightly more noisy than the previous works, one can be quite optimistic that the spin-TOF will in fact be able to take data much more efficiently once the lens tables and software are fully calibrated.

By taking measurements of the four possible combinations of target and sample magnetizations, one could follow the analysis of section 5.3.3 and investigate the separate exchange and spin-orbit contributions to the polarimeter scattering. The spin-orbit contribution should be quite negligible, however, as the 'Planar' scattering geometry is explicitly used here. As described in section 5.3.4 the four measurements could be used to remove any instrumental asymmetry. Unfortunately, the sample manipulator which must be moved horizontally and a large distance vertically to the prep chamber coil position for magnetizing and currently is not able to closely reproduce the previous analysis position. The small change in sample position between measurements with the Fe/W(110) sample magnetized in different directions will have a slight systematic asymmetry which will not allow such an analysis to be trustworthy. This is probably the strongest contribution to the small differences between the upper and lower panels of Figure 6.10. Indeed, because of the high

quality polarimeter target magnetization scheme (section 5.4.4), instrumental asymmetry is likely much smaller than the systematic asymmetry due to the changing sample position.

\subsection{3 $\mathrm{Au}(111)$ surface state}

A surprising and particularly elegant system to observe spin polarized photoemission is the $\mathrm{Au}(111)$ surface. It exhibits a strong $s p$-derived Shockley surface state forming a twodimensional band of electrons confined to the surface within the projected bulk $L$-bandgap. In addition to the interest due to its low dimensionality and nearly-free electron-like behav- 
ior, the surface state has gained a lot of attention as it is the canonical Rashba system showing a complete spin-splitting despite the lack of any ferromagnetic behavior. ${ }^{186,187,188,189}$

In section 3.2 it was shown how spin polarized photoemission can arise from nonmagnetic materials due to spin-orbit coupling. It should be stressed that the spin-orbit coupling generally only splits levels due to their total angular momentum, $J$ (see equations 1.25 and 1.26), and does not actually lift the spin degeneracy of each state. In fact, electronic states in nonmagnetic solids are often required to remain spin degenerate due to the so-called Kramer's degeneracy ${ }^{15}$ which results from a combination of time-reversal and inversion symmetries. This can be seen as follows: time-reversal symmetry requires that $E(k, \uparrow)=E(-k, \downarrow)$, where $E(k, \uparrow)$ is the energy of the state with momentum $k$ and spin-up. In bulk crystals (with a center of inversion), inversion symmetry additionally requires $E(k, \uparrow)=E(-k, \uparrow)$. The combination then requires $E(k, \uparrow)=E(k, \downarrow)$; bulk states must remain spin-degenerate, even in the presence of spin-orbit coupling. This is why the spin polarized photoelectrons described in section 3.2 are only obtained with the use of circularly polarized light to take advantage of selection rules.

The surface, however, lacks inversion symmetry, and so surface states are not subject to Kramer's degeneracy and may in fact be spin-split by spin-orbit coupling. From section 1.2.3 we can rewrite equation 1.20 in terms of the Pauli matrices, substituting $\mathbf{S}=\frac{\hbar}{2} \boldsymbol{\sigma}$ to give

$$
H_{L S}=\frac{e \hbar}{4 m_{e}^{2} c^{2}} \boldsymbol{\sigma} \cdot[\mathbf{p} \times \mathbf{E}]
$$

The electric field can be expressed as the gradient of a potential energy, $V$, as $E=$ $-(1 / e) \nabla V$, allowing the interaction to be written

$$
H_{L S}=\frac{\hbar}{4 m_{e}^{2} c^{2}} \boldsymbol{\sigma} \cdot[\nabla V \times \mathbf{p}]
$$

If one approximates the electrons of the surface state as being a two-dimensional free electron gas, they are only constrained in the $z$-direction giving $\nabla V=\frac{\mathrm{d} V}{\mathrm{~d} z} \hat{z}$, where $V$ is the potential confining the electrons to the surface, and $\hat{z}$ is the unit vector perpendicular to the surface. Confined to the surface, the electrons must occupy states with $\mathbf{p}$ parallel to the surface, with $k$-vectors $\hbar \mathbf{k}_{\|}=\mathbf{p}$. Thus the spin-orbit interaction is reduced to the so-called Rashba 
form of

$$
H_{R}=\frac{\hbar^{2}}{4 m_{e}^{2} c^{2}} \frac{\mathrm{d} V}{\mathrm{~d} z} \boldsymbol{\sigma} \cdot\left(\hat{z} \times \mathbf{k}_{\|}\right)=\alpha_{R} \boldsymbol{\sigma} \cdot\left(\hat{z} \times \mathbf{k}_{\|}\right)
$$

where the Rashba parameter, $\alpha_{R}$, represents the strength of the interaction and is proportional to the surface potential gradient (the atomic spin-orbit strength also significantly contributes to this parameter, which is why it is so readily observed in $\mathrm{Au}$ ).

Without the Rashba term, the parabolic electronic states are given by $E\left(\mathbf{k}_{\|}, \pm \sigma\right)=$ $E_{0}+\hbar^{2} \mathbf{k}_{\|}^{2} /\left(2 m^{*}\right)$, where the $\pm \sigma$ refers to the two possible spin directions, and $m^{*}$ is the effective electron mass. The Rashba perturbation splits the spin-degeneracy as (dropping the $\|$ subscripts from $\mathbf{k}$ for simplicity)

$$
\begin{aligned}
E(\mathbf{k}, \pm \sigma) & =E_{0}+\frac{\hbar^{2} k^{2}}{2 m^{*}} \pm \alpha_{R} k \\
& =E_{0}^{\prime}+\frac{\hbar^{2}(k \pm \Delta k)^{2}}{2 m^{*}},
\end{aligned}
$$

thus resulting in two $100 \%$ polarized parabolic bands, offset from each other in $k_{\|}$by $2 \Delta k$. In the above, $E_{0}^{\prime}=E_{0}-\frac{\hbar^{2}(\Delta k)^{2}}{2 m^{*}}$ and $\Delta k=\alpha_{R} m^{*} / \hbar^{2}$. Inspection of equation 6.4 shows that the spin quantization axis (i.e. the direction of the spin polarization) is perpendicular to both the surface normal, $\hat{z}$, and the momentum, $\mathbf{k}_{\|}$.

ARPES is able to directly observe the splitting of the surface state into two offset parabolic bands, as shown in Figure 6.11(a). The spin polarization from the Rashba model is illustrated by the colored lines highlighting each parabola. Electrons in the red band are $100 \%$ spin polarized into the page, while those in the blue band are $100 \%$ polarized out of the page. The total Hamiltonian is isotropic within $\mathbf{k}_{\|}$, and so the parabolas sweep out two concentric circular Fermi surface pieces, also confirmed by ARPES as shown in Figure 6.11(b). As $\mathbf{P}$ must remain perpendicular to $\mathbf{k}_{\|}$, the polarization directions are tangent to the Fermi surface circles, as shown. This shows that despite the spin structure, there is no net polarization through all $k$-space, and there is no net macroscopic magnetic moment; $k$-resolution is required to observe any spin polarization in the photoemitted electrons. However, with $k$-resolution, spin polarization should be observed in photoelectrons emitted even with linear or unpolarized light.

The band splitting was first experimentally resolved by LaShell et. al. in 1996, ${ }^{186}$ 

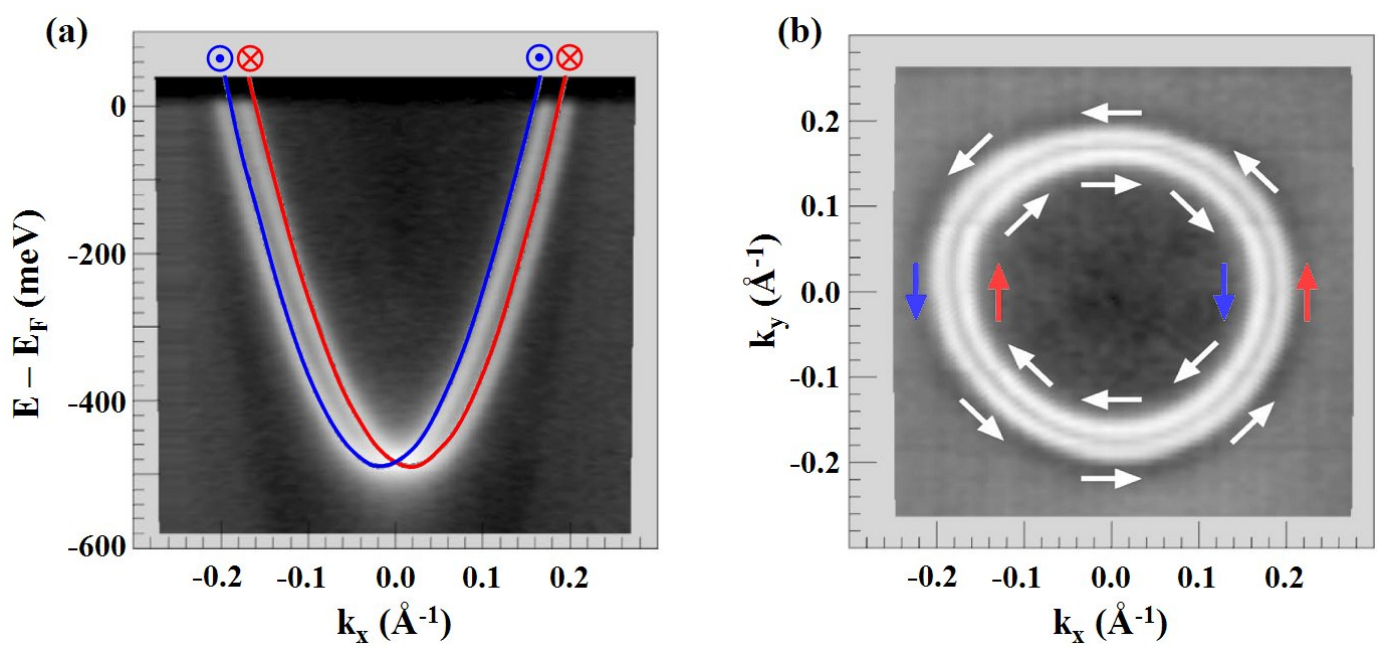

Figure 6.11. ARPES data of $\mathrm{Au}(111) L$-gap surface states, taken with $\mathrm{h} \nu=21.22 \mathrm{eV}$ at $\mathrm{T}=30 \mathrm{~K}$. Figures adapted from ref. 187. (a) Binding energy vs. $k_{x}$ ARPES plot $\left(\right.$ at $\left.k_{y}=0\right)$. Solid curves added as guides to the eye. Red marks band with spin-up (i.e. into the page in this view). Blue marks band with spin-down (i.e. out of the page). (b) ARPES Fermi surface plot. Arrows show direction of spin polarization around each Fermi contour. Red and blue arrows correspond to the spin directions as marked in (a).

proposing the cause to be the Rashba spin-orbit interaction. Full confirmation of this requires spin-resolved ARPES to verify the actual spin-structure. This was first successfully done for the Au surface state by Hoesch et. al. in $2004 .{ }^{189}$ Their spin-resolved EDCs along $k_{x}\left(\right.$ at $\left.k_{y}=0\right)$, corresponding to Figure 6.11(a), are shown in Figure 6.12(a), confirming the spin polarization given by the Rashba model. It is worthwhile to note that the Rashba bandstructure remains spin-degenerate right at $k=0$, resulting in zero spin polarization in photoelectrons emitted perfectly normal to the surface, confirmed in Figure 6.12(a). This then upholds the rule stated in section 3.2, forbidding polarized photoelectrons from nonmagnetic samples illuminated by linear or unpolarized light at normal emission.

The spin-splitting of the $\mathrm{Au}(111)$ surface state makes for a great system for testing a spin-resolved spectrometer for a couple reasons. The required $k$-resolution to observe any spin polarization allows one to prove some degree of angular resolution if spin polarization is observed. Additionally, to ensure any measured asymmetry is truly due to spin polarization, one only has to rotate the Au sample a few degrees to the other side of normal emission to flip the spin polarization of the two EDC peaks (i.e. to go from the bottom to top EDC 

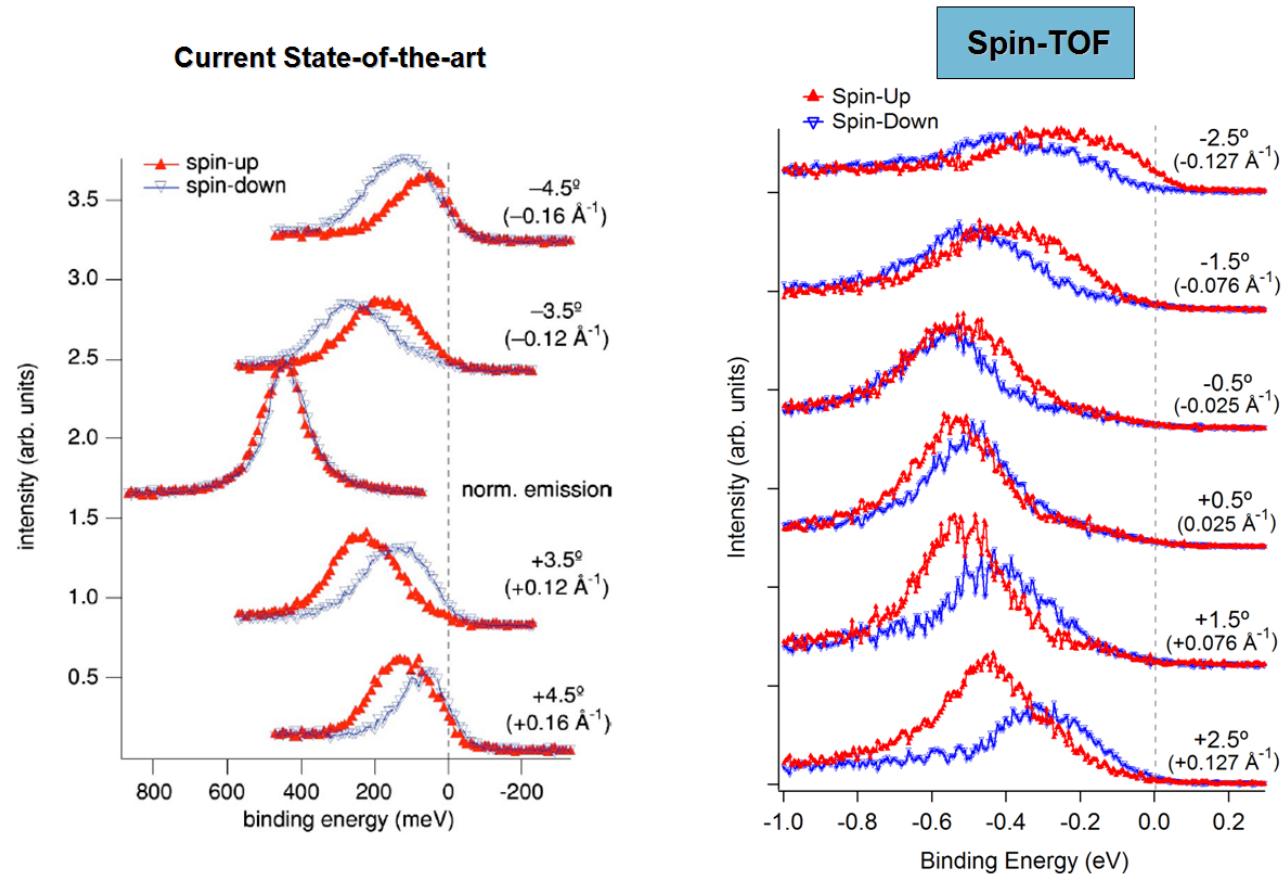

Figure 6.12. Spin-resolved ARPES EDCs of Au(111) surface state along $k_{x}$ (at $k_{y}=0$ ). Left panel is taken from previous work of ref. 189 with $\mathrm{h} \nu=21.1 \mathrm{eV}$ at $\mathrm{T}=150 \mathrm{~K}$. Right panel is taken with the spin-TOF spectrometer with $\mathrm{h} \nu=36.6 \mathrm{eV}$ at room temperature.

in Figure 6.12(a)), in contrast to flipping the magnetization of the ferromagnetic sample as was required in the Fe example of section 6.2.2. Also, a high quality, flat (111) surface is obtainable by evaporation on the W(110) substrate. ${ }^{190,191}$

Performing spin-resolved ARPES with Mode \#2 of the spin-TOF on the Au(111) surface state is shown in Figure 6.12(b). As the entire surface state bandwidth is $\sim 500 \mathrm{meV}$, the full band and EDC lineshapes should fit within the bandpass window (in contrast to the Fe example in section 6.2.2). Six EDC's were taken at the different horizontal emission angles ( $k$-points along $k_{x}$ at $k_{y}=0$ ) shown. The spin polarization clearly flips as the emission angle passes through $0^{\circ}$, strongly supporting the correct operation of the spectrometer. The overall similarity with the previous data shown in panel (a) also supports this. There are some differences in overall lineshapes upon closer inspection. This is likely due to remaining influence of the BPF transmission function in addition to an imperfect Au surface (the $\mathrm{Au}$ thin film should be annealed after growth, or grown with the substrate at elevated temperatures, ${ }^{190}$ however this was not done in the current experiment). 
The spectra also seem slightly noisier than the previous work. This requires a careful analysis for a fair comparison. The data in Figure 6.12(a) were obtained with a SDA/Mott polarimeter combination with $120 \mathrm{meV}$ energy resolution and $1.8^{\circ}$ angular resolution and show 1 data point per $10 \mathrm{meV}$, likely spending $\sim 1$ hour for each EDC. As discussed in sections 6.1.4 and 6.2.2, although the time dispersions of the presently available lens table limit the energy resolution to above $100 \mathrm{meV}$, corrected lens tables would result in the true TOF-limited energy resolution of $\sim 20 \mathrm{meV}$ for the given average kinetic energy. In other words, the statistics were obtained at the same rate as they would be if the analyzer truly achieved $20 \mathrm{meV}$ resolution. For the previous data, this improved resolution would have required 6 times longer, for a total of $\sim 6$ hours per EDC. Also, following the standard rule of thumb of taking 5 data points per resolution width, the spin-TOF data in panel (b) were binned to keep 1 data point per $4 \mathrm{meV}$. This increased bin density would have taken the previous data an additional 2.5 times longer, for a total of $\sim 15$ hours per EDC. The spin-TOF data of panel (b) in fact only took $<30$ minutes per EDC, already suggesting a large increase in total efficiency $(\sim \times 30)$. As argued above in section 6.2 .2 , one must also note that the large beamspot size being used $(\sim 3 \mathrm{~mm})$ likely diminishes the possible count rate quite a bit as the lens system is meant to transmit only a $100 \mu \mathrm{m}$ beamspot through the $\mathrm{BPF}$ and into the polarimeter. When operated with an appropriate beamspot size, the performance of the spin-TOF will be much further improved.

\subsection{Summary}

The spin-TOF spectrometer is formed by integrating the high efficiency exchange polarimeter of Chapter 5 into the TOF electron energy analyzer of Chapter 4 . The spectrometer stands to gain 2-3 orders of magnitude in total efficiency with this combination compared to current state-of-the-art SARPES systems. Despite not being fully calibrated or commissioned, a recent 2-bunch mode period at the ALS was taken advantage of for testing the spectrometer. The spin-TOF demonstrated the strengths of operating in Mode \#1 for convenient sample characterization and preparation. Signal was obtained in Mode 
\#2, and two experiments successfully showed correct spin resolution. Improved lens tables for improved energy resolution, expanded software capabilities for bandpass sweeping, a smaller beamspot for improved resolution and transmission efficiency, and enhanced sample preparation capabilities would all contribute to much improved experimental results. Despite these remaining steps, the spin-TOF already shows very encouraging results exhibiting full spin, angle, and energy resolved spectroscopy at significantly improved efficiencies than other state-of-the-art systems. 


\section{Chapter 7}

\section{Outlook, applications, and conclusions}

\subsection{Future outlook}

Although there are important steps remaining to realize the complete spectrometer, the present successes make the remaining issues exciting ones to tackle. A full set of lens tables must be thoroughly developed for maximizing throughput and minimizing the timing dispersions around the BPF. The photocathode electron source in the lab provides an ideal tool to complete this task. In parallel, a more extensive software package will be developed for handling the lens tables, controlling scanning of the BPF, and acquiring the spin-resolved spectra in an automated and convenient manner. Progress will also be made to more precisely measure the polarimeter scattering target's Sherman function and its energy dependence for accurate polarization analysis. This can be done by utilizing the same spin polarized GaAs photocathode technique as described in section 3.2 and used in the SPLEEM. Work can always continue to develop better scattering targets, both by analyzing effective Sherman functions and FOMs with the SPLEEM and by adapting the preparation recipes in the polarimeter's prep chamber. One specific area which could likely be im- 
proved with further investigation is target stability; surface oxidation ${ }^{171,169}$ and graphene overlayers ${ }^{192,193}$ are particularly intriguing possibilities.

Progress in these areas will certainly be followed by further ALS 2-bunch mode experiments. Demonstration of accurate reproduction of previously published SARPES results will be a necessary early goal. A new horizontal focusing mirror in place at the ALS beamline 12.0.1 will provide a smaller beamspot for much improved experiments. Additionally, room is provided at the new MERLIN beamline for 2-bunch spin-TOF use once construction is complete. The full photon polarization control (any linear, circular, and in between) of the MERLIN elliptically polarized undulator (EPU) will provide additional flexibility for spin-TOF experiments with both magnetic and nonmagnetic systems. Between the scheduled ALS 2-bunch periods, the lab-based $6 \mathrm{eV}$ laser source will provide light for interesting experiments at ultra-low photon energies as well as open the door for exciting pump-probe style time-resolved studies. Once the functionality of the spin-TOF is fully demonstrated, these light sources provide an abundance of exciting applications in SARPES experiments.

\section{2 $\quad$ Exciting applications}

The electron spin is, in one sense, an extremely simple component to a solid's electronic structure. After all, it can only have two possible values in any particular measurement. Despite this inherent simplicity, the spin degree of freedom leads to a vast amount of emergent complexity and technologically useful phenomena, much of which is not well understood or even explored. Manipulation of electron spin and intricate magnetic structures are increasingly important focal points in current materials science research, and yet direct probes of the spin dependence in a material's electronics have remained rare and less feasible and accessible than spin-integrated counterparts. The progress in understanding the role of spin in condensed matter needed in the coming decades will require a strong commitment to the development of powerful spin-sensitive experimental tools. The large successes of ARPES in the past decade in uncovering fascinating momentum-dependent phenomena in the solid state illustrates the key role SARPES could play in future investigations if it can 
be performed with similar resolutions. The spin-TOF represents a significant step in this direction, and I would like to summarize a few areas which I think will be particularly exciting applications for the spectrometer.

\subsubsection{CMR manganites}

The term Colossal Magnetoresistance (CMR) refers to the anomalously large decrease in electrical resistivity with the application of a magnetic field observed in the manganites, a large family of manganese oxide based materials originally studied in the 1950's. ${ }^{194,195}$ The application of this large MR for a wide range of sensor and device technology (in the blossoming field of spintronics ${ }^{17,18}$ ) was quickly seized upon ${ }^{196,197}$ helping to ignite the current renaissance of active research in the field. The manganites, with similar chemical and structural make-up to the HTCs, are now also recognized as prototypical correlated electron systems which require charge, spin, orbital, and lattice degrees of freedom to be simultaneously accounted for. The relevance of the manganite problem to the problems of HTC and correlated systems in general, have greatly boosted the field of CMR manganites, which is now one of the premier areas of research in solid state physics. ${ }^{198,199,200,201}$

CMR manganites feature a field and temperature driven insulating paramagnetic to metallic ferromagnetic phase transition. The low temperature ferromagnetic phase is of course an obvious sign of the importance of spin in this correlated system, and indeed it has a large role in the CMR effect through the double-exchange interaction. ${ }^{202,203,204}$ Of additional interest is that the manganites are predicted to be true "half metals", featuring a gap at $\mathrm{E}_{F}$ for electrons of one spin but not the other. The resulting $100 \%$ spin polarization of charge carriers makes the manganites even more enticing for technology applications. Half-metals in general are extremely desirable for spintronics applications, and predicting, searching, engineering, and characterizing possible half-metals has become an active field. ${ }^{205,206}$ From the wide range of magnetic orderings exhibited by the manganites, ${ }^{200,207}$ to the role that double-exchange plays in the CMR transition, to the expected half-metallicity, it is clear that the spin degree of freedom is quite pertinent to understanding manganite physics. 

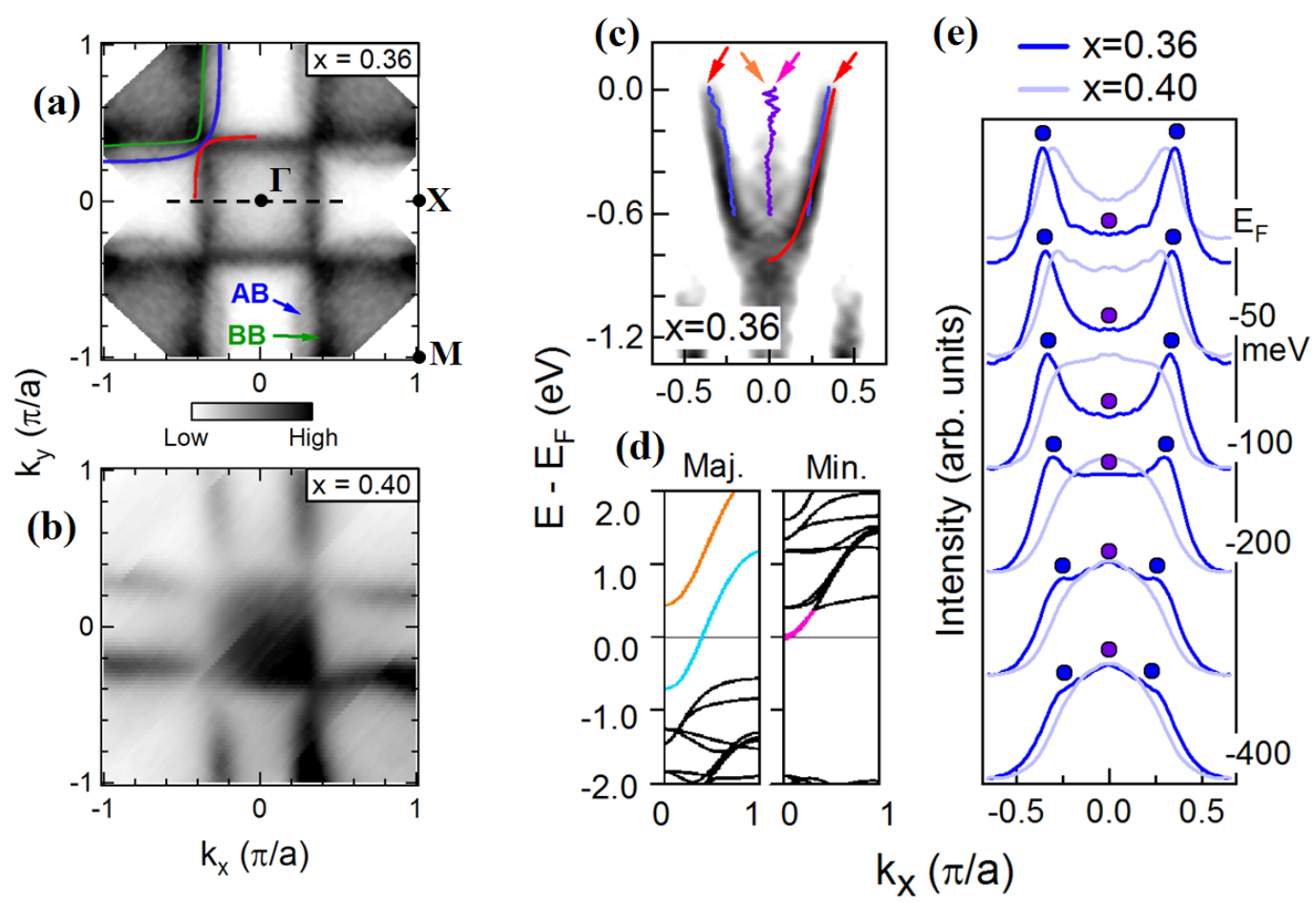

Figure 7.1. ARPES results from the bilayer manganite $\mathrm{La}_{2-2 x} \mathrm{Sr}_{1+2 x} \mathrm{Mn}_{2} \mathrm{O}_{7}$, with $\mathrm{x}=0.36,0.40$. Figures adapted from ref. 208. (a) ARPES derived FS for $\mathrm{x}=0.36$. Calculations of the three bands contributing to the Fermi surface ${ }^{209,54}$ are plotted in green, blue, and red in one quadrant of the Brillouin zone. (b) Corresponding FS of $\mathrm{x}=0.40$. (c) ARPES intensity map (2nd derivative) of binding energy vs. $k_{x}$ directed in $k$-space along dotted line in (a). MDC-extracted dispersions plotted in blue and purple. Theory from (d) plotted in red. (d) Calculations ${ }^{209}$ of majority and minority bandstructure along same direction. (e) MDCs of ARPES intensity maps, similar to (c), for both dopings.

Viewed along the opposite direction, it is also clear that working with the manganites offers a way to experimentally extend our understanding of spin physics in the solid state.

A particularly exciting development was the discovery of CMR in layered manganites formed by aggregates of layered pseudo-2D Mn-O planes. ${ }^{210,207}$ In addition to exhibiting an enhanced CMR and increased correlation effects from reduced dimensionality, the layered manganites opened the door for intensive ARPES study due to their natural cleavage plane providing high-quality in-situ surfaces, in contrast to the 3D manganites. The resulting ARPES studies have made great progress in exploring the bilayer CMR electronic structure. ${ }^{211,212,54,56,213}$ The most notable advances were made possible by the high energy and momentum resolutions offered by current ARPES instrumentation. For instance, 
high resolution experiments allowed resolving the coherent quasiparticle peaks and their renormalized dispersion at $\mathrm{E}_{F} \cdot{ }^{54,56}$ The ability to take this type of data efficiently allowed exploring a large phase space of temperature, momentum, and doping in our own study, ${ }^{208}$ partly shown in Figure 7.1. This particular ARPES study represents the first complete simultaneous mapping of all three predicted components of the bilayer Fermi surface (FS) shown in panel (a), with $36 \%$ chemical doping. This type of mapping exposes an important change taking place in the electronic structure with a slight change of doping, as the FS appears quite different at $40 \%$ doping shown in panel (b). Momentum resolution is also key to obtaining the first mapping of the Mn $3 \mathrm{~d}_{3 z^{2}-r^{2}}$ derived band dispersion responsible for the central electron pocket at $\Gamma$. The ARPES map along the dotted line in panel (a) is shown in panel (b), along with the MDC-extracted dispersions of the three peaks required for fitting. The two outer peaks match the red band in the calculated majority bandstructure in panel (d), however the central, vertical spectral weight (purple dispersion in (c) and evident in the MDCs of panel (e)) could have a number of possible sources. For instance, the spectral weight could be due to the unoccupied orange majority band in the calculated bandstructure (d), or it could be due to the near $\mathrm{E}_{F}$ minority band colored pink.

Despite the progress made, there is much left unresolved. The ARPES work has observed bandstructure near $\mathrm{E}_{F}$ that matches calculations qualitatively well, but are they really $100 \%$ spin majority bands? Does this spin polarization cover all of momentum space, or does it have some unexpected momentum dependence like the quasiparticles studied in ref. 54? Is the spin polarization constant through the CMR doping range, or is there a strong doping dependence? Could the differences in recent ARPES works ${ }^{54,56}$ be linked to differences in spin character? Is the spin polarization the same for both in- and out-of-plane related bands? What is the spin character of the unidentified spectral weight at $\Gamma$ (the two possibilities presented above would have opposite spin character)? Each of these questions and more could have a large impact on our understanding of CMR and our ability to develop successful applications. Each question could also be directly addressed with high efficiency spin resolved ARPES.

The work of J.-H. Park et. al. ${ }^{214,215}$ offers a great example of spin resolved PES be- 

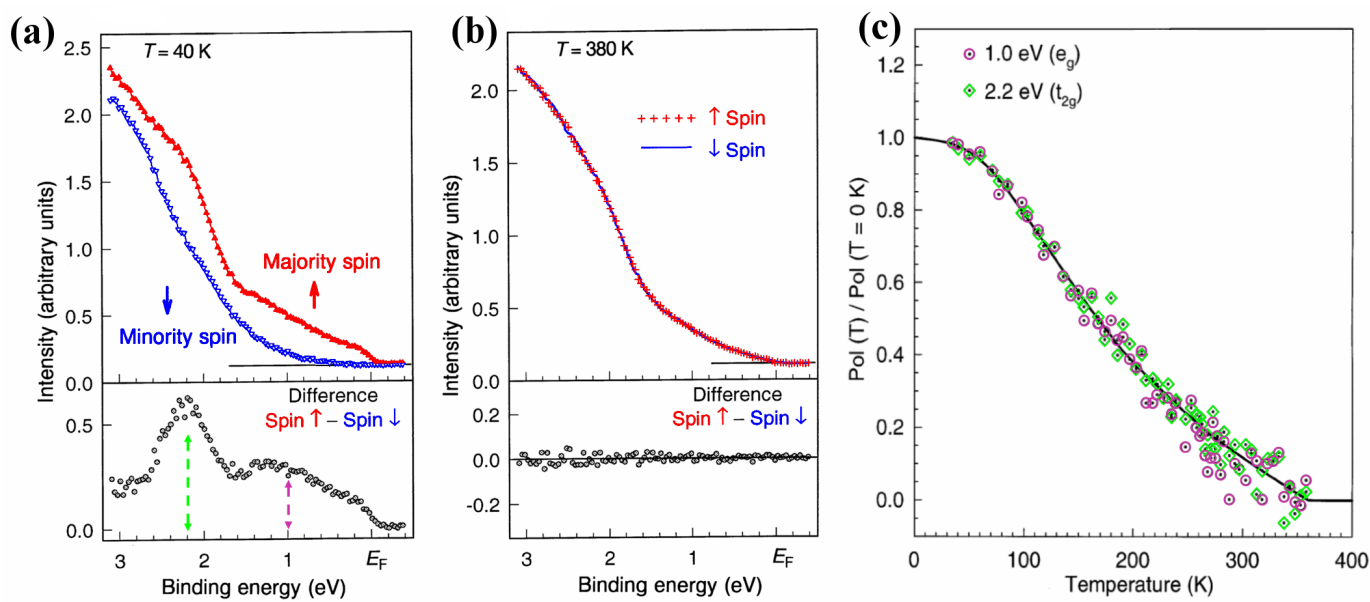

Figure 7.2. Spin resolved PES on $\mathrm{La}_{0.7} \mathrm{Sr}_{0.3} \mathrm{MnO}_{3}$. (a) Spin-resolved PES spectra at 40 $\mathrm{K}\left(\ll \mathrm{T}_{C}\right)$ and corresponding difference spin difference spectrum. (b) The same at $380 \mathrm{~K}$ $\left(>\mathrm{T}_{C}\right)$. Figure adapted from Ref. 214. (c) Full temperature dependence of spin polarization of $\mathrm{e}_{g}$ and $\mathrm{t}_{2 g}$ states measured at 1.0 and $2.2 \mathrm{eV}$ binding energy, respectively. These binding energies correspond to the peaks in the spin difference spectrum at $40 \mathrm{~K}$, marked by the dashed arrows in (a). Figure adapted from Ref. 215.

ing used to investigate the pseudo-cubic CMR manganite, $\mathrm{La}_{0.7} \mathrm{Sr}_{0.3} \mathrm{MnO}_{3}$. Their data is presented in Figure 7.2. Panel (a) shows the angle integrated (the rough sample surface used prohibited angle resolved measurements) PES spectrum at $40 \mathrm{~K}$, decomposed into spin majority and minority channels, with the spin difference spectrum showed below. The measurement suggests that there is finite majority spectral weight at $\mathrm{E}_{F}$ and near zero minority weight from $\mathrm{E}_{F}$ to past $-0.5 \mathrm{eV}$ binding energy, strong evidence in favor of halfmetallicity. The same data taken above $\mathrm{T}_{C}$ at $380 \mathrm{~K}$ is shown in panel (b) and exhibits no spin asymmetry, reflecting the transition to a paramagnetic state. Panel (c) shows the measured spin polarization at two binding energies as a function of temperature, showing a smooth monotonic decrease to zero near $\mathrm{T}_{C}$.

These results are quite promising and hint at the power of spin-resolved photoemission in addressing the physics in these materials. However they do not offer the level of data required to resolve the questions presented above. Due to the inefficiency of the instrumentation, this experiment was performed with no momentum resolution and $0.2 \mathrm{eV}$ energy resolution. The bilayer manganite ARPES works and the vast experience with the HTCs illustrate the need for higher energy resolution and momentum resolution to make substan- 
tial progress in correlated systems. ${ }^{30}$ The spin-TOF analyzer was developed to satisfy these requirements, and addressing these questions will be an extremely interesting project for the spin-TOF. Just as the field of HTC cuprates and ARPES have both grown together in a symbiotic-like relationship, CMR manganites and spin-resolved ARPES may push each other to currently unimaginable frontiers.

\subsubsection{Rashba effect}

One of the most prominent topics in current condensed matter physics is the development of materials and devices capable of utilizing the spin degree of freedom instead of, or in addition to, the charge degree of freedom for information handling. These so-called 'spintronics' applications envision vastly improved performance over traditional electronics in terms of speed, information density, and power consumption. ${ }^{18}$ Central to these aims will be the ability to manipulate single electron spins with applied electric fields in nanoscale and low-dimensional materials and systems. That the manipulation of electron spin through an applied electric field can be made possible is beautifully illustrated by the so-called Rashba effect, introduced in section 6.2.3 and the center of much recent attention in the ARPES community.

In addition to extensive studies of the Rashba splitting of the $\mathrm{Au}(111)$ surface state, ${ }^{186,187,189}$ ARPES has also been used to study similar splitting in the $\mathrm{W}^{216}$ and $\mathrm{Gd}^{217}$ surfaces. Adding to the excitement is the ARPES observed changes in the magnitude of the splitting with rare gas adsorption, ${ }^{218,219,220}$ alkali metal adsorption, ${ }^{221}$ surface alloying, ${ }^{222,220}$ and monolayers of noble metals on $\mathrm{W}$ and Mo surfaces. ${ }^{223}$ All of these demonstrate the possibility of controlling the spin-dependent parameters of the materials, and the number of recent high-profile papers displays the excitement in this area. Despite the excitement, only a few of the above systems have been successfully studied with full spin-resolution, ${ }^{216,189,223}$ with the others remaining unverified.

A very recent, and extremely exciting SARPES work has observed possible Rashba splitting in 2-dimensional graphene sheets. ${ }^{224}$ The apparent ideal electronic properties of 

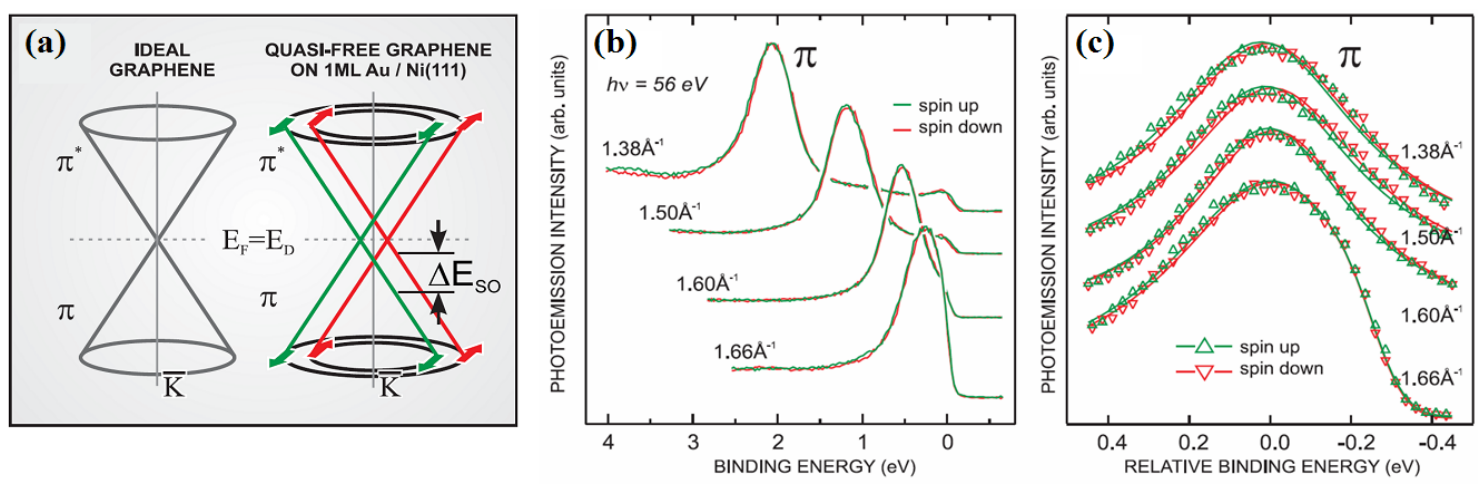

Figure 7.3. Figures taken from ref 224. (a) Model bandstructure of graphene, spin-split by the Rashba effect on the right. (b) SARPES data at $4 k$-points approaching the K-point. (c) Expanded view of data in (b), offset for comparison

graphene has lead to the current explosion of graphene related research and a push for the so-called 'graphene revolution' over the Si-based electronics industry. ARPES, in particular, has played an active role in this research. ${ }^{69,225,226,227}$ It may be that graphene can be useful for spintronics applications as well if its spin transport can be well controlled. Rashba splitting of the conical Dirac dispersions in graphene would result in the spin-polarized bandstructure shown in Figure 7.3(a). SARPES data along one side of the linear dispersion is shown in panel (b). The expanded view of panel (c) exposes a very slight shift in the spin resolved bands, reported as a $13 \pm 3 \mathrm{meV}$ splitting. ${ }^{224}$ This splitting is much larger than expected for a free-standing graphene sheet, and is suggested to be due to interaction with the heavy nuclei of the Au substrate below the graphene layer (again suggesting a form of control over spin properties). The tiny splitting compared to the apparent resolution achieved suggests that much more could be learned if investigated with higher resolutions and higher efficiencies for entire Fermi surface measurements.

High resolution SARPES experiments are the most direct and powerful means for probing the spin structure of these systems. Rashba effects in general will be ideal sample systems for our new spectrometer. Spin-TOF based investigations of spin-splitting in graphene and possible engineered control of this splitting may have extremely widespread impact. 

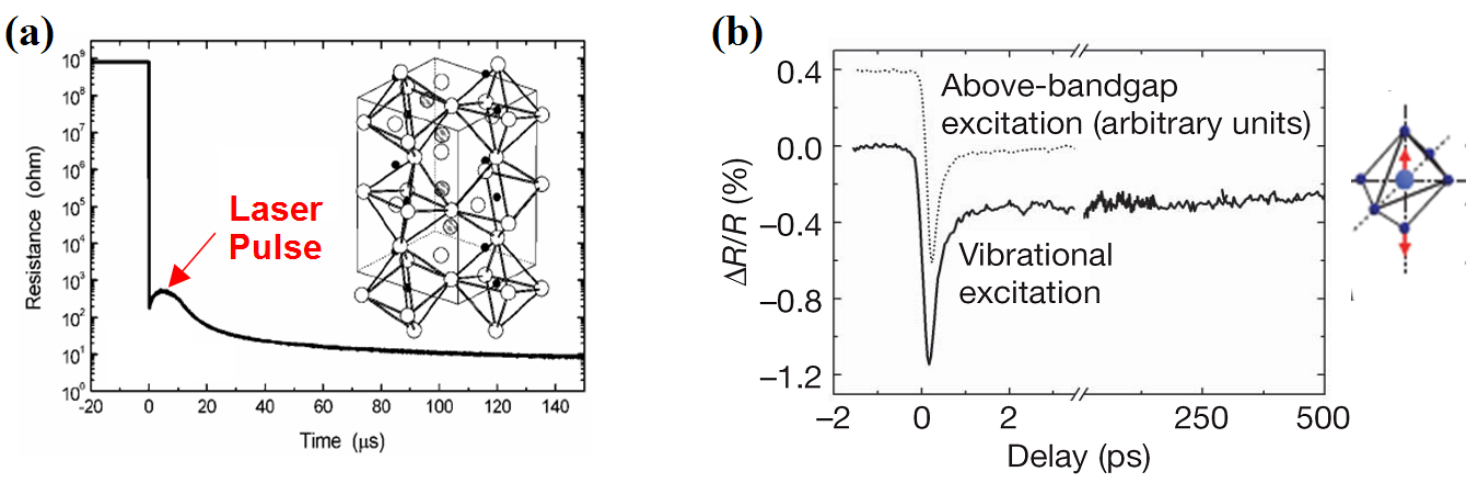

Figure 7.4. Time-resolved responses after photoexcitation of $\operatorname{Pr}_{0.7} \mathrm{Ca}_{0.3} \mathrm{MnO}_{3}$. (a) Resistivity at $30 \mathrm{~K}$ after illumination by $7 \mathrm{~ns}$ pulse of $1064 \mathrm{~nm}$ laser. Figure adapted from ref. 228. (b) Change in $800 \mathrm{~nm}$ reflectivity after excitation by $17.5 \mu \mathrm{m}$ (solid line) and $800 \mathrm{~nm}$ (dotted line) pulses. Figure from ref. 84.

\subsubsection{Time-resolved spin dynamics and phase transitions}

Another area of increasing significance in current experimental physics is the time resolution of fast and ultra-fast dynamics and processes. This pressure for time-resolution is driven by the fundamental importance of time-dependence in physical processes, and by the need for ever increasing speeds in device structures. Although a difficult experiment, time-resolved ARPES has recently begun being performed with great success. ${ }^{79,81,82}$ Magnetization and spin dynamics are of particular technological interest for data processing and storage applications, and spin- and time-resolved photoemission experiments have already been introduced ${ }^{80}$ for these types of investigations. Interesting on its own, this work also adds to the excitement of the possibilities of such experiments performed with the resolutions available with the spin-TOF.

The dynamics of phase transitions are an area of central importance and are the subject of numerous recent high-profile experiments. ${ }^{228,83,79,84,82}$ The works of Fiebig et. al. ${ }^{228}$ and Rini et. al. ${ }^{84}$ investigated the dynamics of the photoinduced and vibrationally induced, respectively, insulator-to-metal transition in the perovskite manganite, $\operatorname{Pr}_{0.7} \mathrm{Ca}_{0.3} \mathrm{MnO}_{3}$. Although this variety of manganite is insulating for all doping levels and all temperatures, it can be driven to the metallic phase by the application of magnetic fields, high pressure, Xrays, and optical photons, ${ }^{228}$ opening the door to studying the dynamics of such transitions. 
Figure 7.4(a) shows the manganite's resistivity as a function of time after exposure to a pulse of $1064 \mathrm{~nm}$ laser light. The data shows a fast and long-lived 8 order of magnitude drop in local resistance between two electrodes, suggesting possible high-speed optical switching devices. Panel (b) shows the manganite's reflectivity as a function of time after exposure to an ultra-fast pulse of $17.5 \mu \mathrm{m}$ laser light, tuned to the sample's Mn-O stretching vibrational mode. Here we see similar insulator-to-metal behavior, this time induced by exciting a specific vibrational mode. In both cases, as metallic behavior is commonly associated with CMR and ferromagnetism in the manganites, these results suggest the ultra-fast formation and control of ferromagnetic domains, an exciting property for spintronics devices. SARPES, performed with time-resolution in similar pump-probe type experiments will be a powerful and direct technique for investigating these and other ultra-fast phase transitions.

As discussed throughout this thesis, because time-resolved pump-probe techniques already require a strictly pulsed excitation light source, TOF instruments (in comparison to the common hemispherical analyzers) become even more ideal as orders of magnitude in efficiency are gained without any comparative loss due to pulsing the source. Indeed, for such time-, spin-, and angle-resolved photoemission experiments, the spin-TOF provides an enormous advantage over other current spectrometers, and will open the door to many fascinating discoveries in the realm of solid state spin dynamics.

\subsection{Conclusions}

This thesis has covered the complete development of a sophisticated electron spectrometer for high resolution spin- and angle-resolved photoemission spectroscopy. The current technology climate is steadily driving interest in the materials sciences towards explicit understanding and manipulation of electron spin and magnetism in the solid state. The obvious tool for furthering these goals is the extension of spin-resolution to the established power of ARPES. In order to achieve the resolutions necessary for SARPES to address the issues of current condensed matter physics, the spectrometer design takes a unique approach to the difficult but powerful experimental technique. A major breakthrough in performance 
is realized by the incorporation of a custom, high performance spin polarimeter into an efficient TOF electron energy analysis scheme. A thorough description of the design, fabrication, assembly, and usage of the entire instrument was given, along with an account of the initial successful results. Although several issues remain to be addressed, the current spectrometer performance promises a bright future of discovery in spin-physics. 


\section{Bibliography}

1. Friedrich, B. \& Herschbach, D. Stern and Gerlach: How a Bad Cigar Helped Reorient Atomic Physics. Phys. Today 56, 53 (2003).

2. Estermann, I. \& Foner, S. N. History of molecular beam research: Personal reminiscences of the important evolutionary period 19191933. Am. J. Phys. 43, 661 (1975).

3. Born, M. My Life: Recollections of a Nobel Laureate (Scribner, New York, 1978).

4. Stern, O. Ein Weg zur experimentellen Prufung Richtungsquantelung im Magnet feld. Z. Phys. 7, 249 (1921).

5. Gerlach, W. \& Stern, O. Der experimentelle Nachweis der Richtungsquantelung im Magnetfeld. Z. Phys. 9, 349 (1922).

6. Goudsmit, S. The discovery of electron spin. http://132.229.227.250/history/spin/goudsmit.html (1971). Lecture, translated by van der Waals, J. H.

7. Uhlenbeck, G. E. \& Goudsmit, S. Ersetzung der Hypothese von unmechanischen Zwang durch eine Forderung bezuglich des inneren Verhaltens jedes einzelnen Elektrons. Naturwissenschaften 13, 953 (1925).

8. Uhlenbeck, G. E. \& Goudsmit, S. Spinning Electrons and the Structure of Spectra. Nature 117, 264 (1926).

9. Thomas, L. H. The Motion of the Spinning Electron. Nature 117, 514 (1926).

10. Ballentine, L. E. Quantum Mechanics - A Modern Development (World Scientific Publishing, 1998).

11. Pauli, W. The Connection Between Spin and Statistics. Phys. Rev. 58, 716 (1940).

12. Duck, I. \& Sudarshan, E. C. G. Toward an understanding of the spin-statistics theorem. Am. J. Phys. 66, 284 (1998).

13. Ashcroft, N. W. \& Mermin, N. D. Solid State Physics (Brooks Cole, 1976).

14. Morrish, A. H. The Physical Principles of Magnetism (IEEE Press, 2001).

15. Sakurai, J. J. Modern Quantum Mechanics (Addison-Wesley, 1994).

16. Feder, R. (ed.). Polarized Electrons in Surface Physics (World Scientific, 1985). 
17. Prinz, G. A. Magnetoelectronics. Science 282, 1660 (1998).

18. Wolf, S. A. et al. Spintronics: A Spin-Based Electronics Vision for the Future. Science 294, 1488 (2001).

19. Tjeng, L. H. et al. Spin-Resolved Photoemission on Anti-Ferromagnets: Direct Observation of Zhang-Rice Singlets in CuO. Phys. Rev. Lett. 78, 1126 (1997).

20. Tjeng, L. H., Brookes, N. B. \& Sinkovic, B. Spin-resolved photoelectron spectroscopy on cuprate systems. J. Electron Spectrosc. Relat. Phenom. 117-118, 189 (2001).

21. Brookes, N. B., Ghiringhelli, G., Tjernberg, O., Tjeng, L. H., Mizokawa, T., Li, T. W. \& Menovsky, A. A. Detection of Zhang-Rice Singlets Using Spin-Polarized Photoemission. Phys. Rev. Lett. 87, 237003 (2001).

22. Kisker, E. Metallic Magnetism (Springer-Verlag, 1987).

23. Johnson, P. D. Spin Polarized Photoemission. Annu. Rev. Mat. Sci. 25, 455 (1995).

24. Johnson, P. D. Spin-polarized photoemission. Rep. Prog. Phys. 60, 1217 (1997).

25. Siegbahn, K. Nobel Lectures in Physics 1981 - 1990 (World Scientific, 1993).

26. Hufner, S. Photoelectron Spectroscopy: Principles and applications (Springer-Verlag, 2003), 3rd edn.

27. Kevan, S. D. (ed.). Angle-Resolved Photoemission (Elsevier, 1992).

28. Himpsel, F. J. Angle-resolved measurements of the photoemission of electrons in the study of solids. Advances in Physics 32, 1 (1983).

29. Hfner, S., Claessen, R., Reinert, F., Straub, T., Strocov, V. N. \& Steiner, P. Photoemission spectroscopy in metals:: band structure-Fermi surface-spectral function. $J$. Electron Spectrosc. Relat. Phenom. 100, 191 (1999).

30. Damascelli, A., Hussain, Z. \& Shen, Z.-X. Angle-resolved photoemission studies of the cuprate superconductors. Rev. Mod. Phys. 75, 473 (2003).

31. Bonzel, H. P. \& Kleint, C. On the history of photoemission. Prog. Surf. Sci. 49, 107 (1995).

32. Hertz, H. Ueber einen Einfluss des ultravioletten Lichtes auf die electrische Entladung. Annalen der Physik und Chemie 267, 983 (1887).

33. Lenard, P. Ueber die lichtelektrische Wirkung. Ann. Phys. (Leipzig) 313, 149 (1902).

34. Millikan, R. A. A Direct Photoelectric Determination of Planck's "h". Phys. Rev. 7, 355 (1916).

35. Einstein, A. ber einen die Erzeugung und Verwandlung des Lichtes betreffenden heuristischen Gesichtspunkt. Ann. Phys. (Leipzig) 322, 132 (1905).

36. Shirley, D. A. High-Resolution X-Ray Photoemission Spectrum of the Valence Bands of Gold. Phys. Rev. B 5, 4709 (1972). 
37. Li, X., Zhang, Z. \& Henrich, V. E. Inelastic electron background function for ultraviolet photoelectron spectra. J. Electron Spectrosc. Relat. Phenom. 63, 253 (1993).

38. Axelson, G. et al. New Approach to Structure Studies in Organic Chemistry. Nature 213, 70 (1967).

39. Nordling, C., Sokolowski, E. \& Siegbahn, K. Precision Method for Obtaining Absolute Values of Atomic Binding Energies. Phys. Rev. 105, 1676 (1957).

40. Fahlman, A., Hamrin, K., Hedman, J., Nordberg, R., Nordling, C. \& Siegbahn, K. Electron Spectroscopy and Chemical Binding. Nature 210, 4 (1966).

41. Siegbahn, K. et al. ESCA Atomic, Molecular and Solid State Structure Studied by Means of Electron Spectroscopy (Almqvist and Wiksells, 1967).

42. Siegbahn, K. et al. ESCA Applied to Free Molecules (North-Holland, 1969).

43. Spicer, W. E. \& Berglund, C. N. d Band of Copper. Phys. Rev. Lett. 12, 9 (1964).

44. Berglund, C. N. \& Spicer, W. E. Photoemission Studies of Copper and Silver: Experiment. Phys. Rev. 136, A1044 (1964).

45. Berglund, C. N. \& Spicer, W. E. Photoemission Studies of Copper and Silver: Theory. Phys. Rev. 136, A1030 (1964).

46. Smith, N. V., Thiry, P. \& Petroff, Y. Photoemission linewidths and quasiparticle lifetimes. Phys. Rev. B 47, 15476 (1993).

47. Rossnagel, K. et al. Three-dimensional Fermi surface determination by angle-resolved photoelectron spectroscopy. Phys. Rev. B 63, 125104 (2001).

48. Kane, E. O. Implications of Crystal Momentum Conservation in Photoelectric Emission for Band-Structure Measurements. Phys. Rev. Lett. 12, 97 (1964).

49. Smith, N. V., Traum, M. M. \& Di Salvo, F. J. Mapping energy bands in layer compounds from the angular dependence of ultraviolet photoemission. Solid State Commun. 15, 211 (1974).

50. Traum, M. M., Smith, N. V. \& Di Salvo, F. J. Angular Dependence of Photoemission and Atomic Orbitals in the Layer Compound 1 T-TaSe2. Phys. Rev. Lett. 32, 1241 (1974).

51. Smith, N. V. \& Traum, M. M. Angular-resolved ultraviolet photoemission spectroscopy and its application to the layer compounds $\mathrm{TaSe}_{2}$ and $\mathrm{TaS}_{2}$. Phys. Rev. B 11, 2087 (1975).

52. Koralek, J. D. et al. Laser Based Angle-Resolved Photoemission, the Sudden Approximation, and Quasiparticle-Like Spectral Peaks in $\mathrm{Bi}_{2} \mathrm{Sr}_{\text {sub2 }} \mathrm{CaCu}_{\text {sub2 }} \mathrm{O}_{8+\delta}$. Phys. Rev. Lett. 96, 017005 (2006).

53. Mahan, G. D. Many-Particle Physics (Kluwer Academic, 2000), 3rd edn.

54. Mannella, N. et al. Nodal quasiparticle in pseudogapped colossal magnetoresistive manganites. Nature 438, 474 (2005). 
55. Lanzara, A. et al. Evidence for ubiquitous strong electron-phonon coupling in hightemperature superconductors. Nature 412, 510 (2001).

56. Sun, Z. et al. Quasiparticlelike peaks, kinks, and electron-phonon coupling at the (pi, 0) regions in the CMR oxide $\mathrm{La}_{2-2 x} \mathrm{Sr}_{1+2 x} \mathrm{Mn}_{2} \mathrm{O}_{7}$. Phys. Rev. Lett. 97, 056401 (2006).

57. Gweon, G. H. Looking through the lattice - Unraveling the complexity of the cuprate problem (unpublished).

58. Feng, D. L. et al. Electronic excitations near the Brillouin zone boundary of $\mathrm{Bi}_{2} \mathrm{Sr}_{2} \mathrm{CaCu}_{2} \mathrm{O}_{8+\delta}$. Phys. Rev. B 65, 220501 (2002).

59. Kordyuk, A. A. et al. Origin of the Peak-Dip-Hump Line Shape in the Superconducting-State $(\pi, 0)$ Photoemission Spectra of $\mathrm{Bi}_{2} \mathrm{Sr}_{2} \mathrm{CaCu}_{2} \mathrm{O}_{8}$. Phys. Rev. Lett. 89, 077003 (2002).

60. Borisenko, S. V. et al. Anomalous Enhancement of the Coupling to the Magnetic Resonance Mode in Underdoped Pb-Bi2212. Phys. Rev. Lett. 90, 207001 (2003).

61. Chuang, Y. D. et al. Bilayer splitting and coherence effects in optimal and underdoped $\mathrm{Bi}_{2} \mathrm{Sr}_{2} \mathrm{CaCu}_{2} \mathrm{O}_{8+\delta}$. Phys. Rev. B 69, 094515 (2004).

62. Borisenko, S. V. et al. Parity of the Pairing Bosons in a High-Temperature $\mathrm{Pb}-\mathrm{Bi}_{2} \mathrm{Sr}_{2} \mathrm{CaCu}_{2} \mathrm{O}_{8}$ Bilayer Superconductor by Angle-Resolved Photoemission Spectroscopy. Phys. Rev. Lett. 96, 067001 (2006).

63. Zabolotnyy, V. B. et al. Disentangling surface and bulk photoemission using circularly polarized light. Phys. Rev. B 76, 024502 (2007).

64. Dessau, D. S., Chuang, Y. D., Gromko, A., Saitoh, T., Kimura, T. \& Tokura, Y. Electronic structure of CMR oxides: high resolution electron-spectroscopic studies and a pseudogap. J. Electron Spectrosc. Relat. Phenom. 117, 265 (2001).

65. Shen, Z.-X. et al. Anomalously large gap anisotropy in the a-b plane of $\mathrm{Bi}_{2} \mathrm{Sr}_{2} \mathrm{CaCu}_{2} \mathrm{O}_{8+\delta}$. Phys. Rev. Lett. 70, 1553 (1993).

66. Gweon, G.-H., Sasagawa, T., Zhou, S., Graf, J., Takagi, H., Lee, D.-H. \& Lanzara, A. An unusual isotope effect in a high-transition-temperature superconductor. Nature 430, 187 (2004).

67. Yang, W. L. et al. Band Structure and Fermi Surface of Electron-Doped C60 Monolayers. Science 300, 303 (2003).

68. Zhou, S. Y. et al. First direct observation of Dirac fermions in graphite. Nat. Phys. 2, 595 (2006).

69. Ohta, T., Bostwick, A., Seyller, T., Horn, K. \& Rotenberg, E. Controlling the Electronic Structure of Bilayer Graphene. Science 313, 951 (2006).

70. Hsieh, D., Qian, D., Wray, L., Xia, Y., Hor, Y. S., Cava, R. J. \& Hasan, M. Z. A topological Dirac insulator in a quantum spin Hall phase. Nature 452, 970 (2008).

71. Roy, D. \& Tremblay, D. Design of electron spectrometers. Rep. Prog. Phys. 1621 (1990). 
72. Smith, N. \& Kevan, S. General instrumentation considerations in electron and ion spectroscopies using synchrotron radiation. Nucl. Instrum. Methods 195, 309 (1982).

73. Kevan, S. D. Design of a high-resolution angle-resolving electron energy analyzer. Rev. Sci. Instrum. 54, 1441 (1983).

74. Mårtensson, N., Baltzer, P., Brhwiler, P. A., Forsell, J. O., Nilsson, A., Stenborg, A. \& Wannberg, B. A very high resolution electron spectrometer. J. Electron Spectrosc. Relat. Phenom. 70, 117 (1994).

75. Reinert, F., Nicolay, G., Schmidt, S., Ehm, D. \& Hfner, S. Direct measurements of the L-gap surface states on the (111) face of noble metals by photoelectron spectroscopy. Phys. Rev. B 63, 115415 (2001).

76. Dessau, D. S. et al. Key features in the measured band structure of Bi2Sr2CaCu2O8+ delta : Flat bands at EF and Fermi surface nesting. Phys. Rev. Lett. 71, 2781 (1993).

77. Bogdanov, P. V. et al. Evidence for an Energy Scale for Quasiparticle Dispersion in $\mathrm{Bi}_{2} \mathrm{Sr}_{2} \mathrm{CaCu}_{2} \mathrm{O}_{8}$. Phys. Rev. Lett. 85, 2581 (2000).

78. Shen, K. M. Angle Resolved Photoemission Spectroscopy Studies of the Mott Insulator to Superconductor Evolution of $\mathrm{Ca}_{2-x} \mathrm{Na}_{x} \mathrm{CuO}_{2} \mathrm{Cl}_{2}$. Ph.D. thesis, Stanford University (2005).

79. Perfetti, L. et al. Time Evolution of the Electronic Structure of $1 \mathrm{~T}-\mathrm{TaS}_{2}$ through the Insulator-Metal Transition. Phys. Rev. Lett. 97, 067402 (2006).

80. Cinchetti, M. et al. Spin-Flip Processes and Ultrafast Magnetization Dynamics in Co: Unifying the Microscopic and Macroscopic View of Femtosecond Magnetism. Phys. Rev. Lett. 97, 177201 (2006).

81. Perfetti, L., Loukakos, P. A., Lisowski, M., Bovensiepen, U., Eisaki, H. \& Wolf, M. Ultrafast Electron Relaxation in Superconducting $\mathrm{Bi}_{2} \mathrm{Sr}_{2} \mathrm{CaCu}_{2} \mathrm{O}_{8+\delta}$ by Time-Resolved Photoelectron Spectroscopy. Phys. Rev. Lett. 99, 197001 (2007).

82. Schmitt, F. et al. Transient Electronic Structure and Melting of a Charge Density Wave in TbTe3. Science 321, 1649 (2008).

83. Cavalleri, A. et al. Band-Selective Measurements of Electron Dynamics in $\mathrm{VO}_{2}$ Using Femtosecond Near-Edge X-Ray Absorption. Phys. Rev. Lett. 95, 067405 (2005).

84. Rini, M. et al. Control of the electronic phase of a manganite by mode-selective vibrational excitation. Nature 449, 72 (2007).

85. Feder, R. Polarized Electrons in Surface Physics (World Scientific, 1985).

86. Feuchtwang, T. E., Cutler, P. H. \& Schmit, J. A review of the theoretical and experimental analyses of electron spin polarization in ferromagnetic transition metals, : I. Field emission, photoemission, magneto-optic Kerr effect and tunneling. Surf. Sci. 75, 401 (1978).

87. Unguris, J., Pierce, D. T., Galejs, A. \& Celotta, R. J. Spin and Energy Analyzed Secondary Electron Emission from a Ferromagnet. Phys. Rev. Lett. 49, 72 (1982). 
88. Scheinfein, M. R., Unguris, J., Kelley, M. H., Pierce, D. T. \& Celotta, R. J. Scanning electron microscopy with polarization analysis (SEMPA). Rev. Sci. Instrum. 61, 2501 (1990).

89. Meier, F. Polarized Electrons in Surface Physics (World Scientific, 1985).

90. Fano, U. Spin Orientation of Photoelectrons Ejected by Circularly Polarized Light. Phys. Rev. 178, 131 (1969).

91. Kessler, J. \& Lorenz, J. Experimental Verification of the Fano Effect. Phys. Rev. Lett. 24, 87 (1970).

92. Pierce, D. T., Meier, F. \& Zurcher, P. Negative electron affinity GaAs: A new source of spin-polarized electrons. Appl. Phys. Lett. 26, 670 (1975).

93. Pierce, D. T. \& Meier, F. Photoemission of spin-polarized electrons from GaAs. Phys. Rev. B 13, 5484 (1976).

94. Pierce, D. T., Celotta, R. J., Wang, G.-C., Unertl, W. N., Galejs, A., Kuyatt, C. E. \& Mielczarek, S. R. The GaAs spin polarized electron source. Rev. Sci. Instrum. 51, 478 (1980).

95. Kirschner, J. Polarized Electrons at Surfaces, vol. 106 of Springer Tracts in Modern Physics (Springer, 1985).

96. Heinzmann, U. \& Schonhense, G. Polarized Electrons in Surface Physics (World Scientific, 1985).

97. Ghiringhelli, G., Tjeng, L. H., Tanaka, A., Tjernberg, O., Mizokawa, T., de Boer, J. L. \& Brookes, N. B. 3d spin-orbit photoemission spectrum of nonferromagnetic materials: The test cases of $\mathrm{CoO}$ and Cu. Phys. Rev. B 66, 075101 (2002).

98. Sinkovic, B. et al. Local Electronic and Magnetic Structure of Ni below and above TC: A Spin-Resolved Circularly Polarized Resonant Photoemission Study. Phys. Rev. Lett. 79, 3510 (1997).

99. Brookes, N. B. et al. Study of magnetism using circularly polarized soft X-rays. J. Electron Spectrosc. Relat. Phenom. 92, 11 (1998).

100. Kisker, E., Schrder, K., Campagna, M. \& Gudat, W. Temperature Dependence of the Exchange Splitting of Fe by Spin-Resolved Photoemission Spectroscopy with Synchrotron Radiation. Phys. Rev. Lett. 52, 2285 (1984).

101. Fedorov, A. V., Valla, T., Liu, F., Johnson, P. D., Weinert, M. \& Allen, P. B. Spinresolved photoemission study of photohole lifetimes in ferromagnetic gadolinium. Phys. Rev. B 65, 212409 (2002).

102. Busch, G., Campagna, M., Cotti, P. \& Siegmann, H. C. Observation of Electron Polarization in Photoemission. Phys. Rev. Lett. 22, 597 (1969).

103. Clauberg, R., Gudat, W., Kisker, E., Kuhlmann, E. \& Rothberg, G. M. Nature of the Resonant 6-eV Satellite in Ni: Photoelectron Spin-Polarization Analysis. Phys. Rev. Lett. 47, 1314 (1981). 
104. Kirschner, J., Feder, R. \& Wendelken, J. F. Electron Spin Polarization in Energy- and Angle-Resolved Photoemission from W(001): Experiment and Theory. Phys. Rev. Lett. 47, 614 (1981).

105. Huang, D.-J., Lee, J.-Y., Suen, J.-S., Mulhollan, G. A., Andrews, A. B. \& Erskine, J. L. Adapting a compact Mott spin polarimeter to a large commercial electron energy analyzer for spin-polarized electron spectroscopy. Rev. Sci. Instrum. 64, 3474 (1993).

106. Fedorov, A. V., Valla, T., Huang, D. J., Reisfeld, G., Loeb, F., Liu, F. \& Johnson, P. D. Spin polarized photoemission studies of the Gd(0001) surface. J. Electron Spectrosc. Relat. Phenom. 92, 19 (1998).

107. Ghiringhelli, G., Larsson, K. \& Brookes, N. B. High-efficiency spin-resolved and spinintegrated electron detection: Parallel mounting on a hemispherical analyzer. Rev. Sci. Instrum. 70, 4225 (1999).

108. Hoesch, M., Greber, T., Petrov, V. N., Muntwiler, M., Hengsberger, M., Auwrter, W. \& Osterwalder, J. Spin-polarized Fermi surface mapping. J. Electron Spectrosc. Relat. Phenom. 124, 263 (2002).

109. Rehn, V. Time-resolved spectroscopy in synchrotron radiation. Nucl. Instrum. Methods 177, 193 (1980).

110. Monahan, K. \& Rehn, V. Exploiting the unique time-structure of synchrotron radiation at SSRL. Nucl. Instrum. Methods 152, 255 (1978).

111. Munro, I. \& Schwentner, N. Time resolved spectroscopy using synchrotron radiation. Nucl. Instrum. Methods in Phys. Research 208, 819 (1983).

112. Bachrach, R. Z., Brown, F. C. \& Hagstrom, S. B. M. Photoelectron spectroscopy by time - of - flight technique using synchrotron radiation. J. Vac. Sci. Technol. 12, 309 (1975).

113. Bachrach, R. Z., Skibowski, M. \& Brown, F. C. Angle-Resolved Photoemission from TiSe2 Using Synchrotron Radiation. Phys. Rev. Lett. 37, 40 (1976).

114. White, M. G., Rosenberg, R. A., Gabor, G., Poliakoff, E. D., Thornton, G., Southworth, S. H. \& Shirley, D. A. Time-of-flight photoelectron spectroscopy of gases using synchrotron radiation. Rev. Sci. Instrum. 50, 1268 (1979).

115. Becker, U. et al. Subshell photoionization of Xe between 40 and $1000 \mathrm{eV}$. Phys. Rev. A 39, 3902 (1989).

116. Mller, N. et al. Spin resolved auger electron spectroscopy after photoexcitation with circularly polarized radiation from the BESSY crossed undulator. J. Electron Spectrosc. Relat. Phenom. 72, 187 (1995).

117. Snell, G. et al. Spin Polarized Auger Electrons: The Xe M4,5N4,5N4,5 Case. Phys. Rev. Lett. 76, 3923 (1996).

118. Snell, G. et al. Complete Description of the Xe 4d Photoionization by Spin-Resolved Photoelectron and Auger Spectroscopy. Phys. Rev. Lett. 82, 2480 (1999). 
119. Snell, G., Langer, B., Young, A. T. \& Berrah, N. Spin-polarization measurements of the krypton M4,5NN and xenon N4,5OO Auger electrons: Orientation and intrinsic parameters. Phys. Rev. A 66, 022701 (2002).

120. Turri, G. et al. Probing the Molecular Environment Using Spin-Resolved Photoelectron Spectroscopy. Phys. Rev. Lett. 92, 013001 (2004).

121. Turri, G., Lohmann, B., Langer, B., Snell, G., Becker, U. \& Berrah, N. Spin polarization of the $\mathrm{Ar}^{*} 2 \mathrm{p}_{1 / 2}^{-1} 4 \mathrm{~s}$ and $2 \mathrm{p}_{1 / 2}^{-1} 3 \mathrm{~d}$ resonant Auger decay. Journal of Physics B: Atomic, Molecular and Optical Physics 3453 (2007).

122. Wiedenhoeft, M., Canton, S. E., Wills, A. A., Gorczyca, T., Viefhaus, J., Becker, U. $\&$ Berrah, N. Coincident energy and angular distributions in xenon $4 \mathrm{~d}_{5 / 2}$ inner-shell double photoionization. Journal of Physics B: Atomic, Molecular and Optical Physics 41, 095202 (2008).

123. Hemmers, O., Whitfield, S. B., Glans, P., Wang, H., Lindle, D. W., Wehlitz, R. \& Sellin, I. A. High-resolution electron time-of-flight apparatus for the soft x-ray region. Rev. Sci. Instrum. 69, 3809 (1998).

124. Bokor, J., Haight, R., Storz, R. H., Stark, J., Freeman, R. R. \& Bucksbaum, P. H. Time- and angle-resolved photoemission study of $\operatorname{InP}(110)$. Phys. Rev. B 32, 3669 (1985).

125. Haight, R., Bokor, J., Stark, J., Storz, R. H., Freeman, R. R. \& Bucksbaum, P. H. Picosecond Time-Resolved Photoemission Study of the $\operatorname{InP}(110)$ Surface. Phys. Rev. Lett. 54, 1302 (1985).

126. Bokor, J., Storz, R., Freeman, R. R. \& Bucksbaum, P. H. Picosecond Surface Electron Dynamics on Photoexcited Si(111) (2×1) Surfaces. Phys. Rev. Lett. 57, 881 (1986).

127. Haight, R., Bokor, J., Freeman, R. R. \& Bucksbaum, P. H. Time and angle resolved ultraviolet photoemission spectroscopy studies of single crystal surface and interfaces. J. Vac. Sci. Technol. A 4, 1481 (1986).

128. Haight, R., Silberman, J. A. \& Lilie, M. I. Novel system for picosecond photoemission spectroscopy. Rev. Sci. Instrum. 59, 1941 (1988).

129. Karlsson, H. S., Chiaia, G. \& Karlsson, U. O. A system for time- and angle-resolved photoelectron spectroscopy based on an amplified femtosecond titanium:sapphire laser system. Rev. Sci. Instrum. 67, 3610 (1996).

130. Moos, G., Gahl, C., Fasel, R., Wolf, M. \& Hertel, T. Anisotropy of Quasiparticle Lifetimes and the Role of Disorder in Graphite from Ultrafast Time-Resolved Photoemission Spectroscopy. Phys. Rev. Lett. 87, 267402 (2001).

131. Padowitz, D. F., Merry, W. R., Jordan, R. E. \& Harris, C. B. Two-photon photoemission as a probe of electron interactions with atomically thin dielectric films on metal surfaces. Phys. Rev. Lett. 69, 3583 (1992).

132. McNeill, J. D., Lingle, R. L., Ge, N.-H., Wong, C. M., Jordan, R. E. \& Harris, C. B. Dynamics and Spatial Distribution of Electrons in Quantum Wells at Interfaces Determined by Femtosecond Photoemission Spectroscopy. Phys. Rev. Lett. 79, 4645 (1997). 
133. Harris, C. B., Ge, N.-H., Lingle, R. L., McNeill, J. D. \& Wong, C. M. Femtosecond Dynamics of Electrons on Surfaces and at Interfaces. Annual Review of Physical Chemistry 48, 711 (1997).

134. Wiza, J. L. Microchannel plate detectors. Nucl. Instrum. Methods 162, 587 (1979).

135. Burle Long Life MCP Selection Guide (2006). Http://www.burle.com/cgibin/byteserver.pl/pdf/EP107.pdf.

136. Paulus, T. J. Timing electronics and fast timing methods with scintillation detectors. IEEE Trans. Nuc. Sci. 32, 1242 (1985).

137. Vredenborg, A., Roeterdink, W. G. \& Janssen, M. H. M. A photoelectron-photoion coincidence imaging apparatus for femtosecond time-resolved molecular dynamics with electron time-of-flight resolution of $\sigma=18 \mathrm{ps}$ and energy resolution $\Delta E / E=3.5 \%$. Rev. Sci. Instrum. 79, 063108 (2008).

138. Trevor, D. J., Van Woerkom, L. D. \& Freeman, R. R. A parabolic mirror time-of-flight electron energy analyzer. Rev. Sci. Instrum. 60, 1051 (1989).

139. Lebedev, G., Jozwiak, C., Andresen, N., Lanzara, A. \& Hussain, Z. TOF electron energy analyzer for spin and angular resolved photoemission spectroscopy. Physics Procedia 1, 413 (2008).

140. Kessler, J. Polarized Electrons (Springer, 1976).

141. Batelaan, H., Gay, T. J. \& Schwendiman, J. J. Stern-Gerlach Effect for Electron Beams. Phys. Rev. Lett. 79, 4517 (1997).

142. Sherman, N. Coulomb Scattering of Relativistic Electrons by Point Nuclei. Phys. Rev. 103, 1601- (1956).

143. Motz, J. W., Olsen, H. \& Koch, H. W. Electron Scattering without Atomic or Nuclear Excitation. Rev. Mod. Phys. 36, 881 (1964).

144. Gay, T. J. \& Dunning, F. B. Mott electron polarimetry. Rev. Sci. Instrum. 63, 1635 (1992).

145. Burnett, G. C., Monroe, T. J. \& Dunning, F. B. High-efficiency retarding-potential Mott polarization analyzer. Rev. Sci. Instrum. 65, 1893 (1994).

146. Neufeld, D. D., Aliabadi, H. \& Dunning, F. B. Compact retarding-potential Mott polarimeter. Rev. Sci. Instrum. 78, 025107 (2007).

147. Snell, G., Viefhaus, J., Dunning, F. B. \& Berrah, N. Microsphere plate detectors used with a compact Mott polarimeter for time-of-flight studies. Rev. Sci. Instrum. 71, 2608 (2000).

148. O’Neill, M. R., Kalisvaart, M., Dunning, F. B. \& Walters, G. K. Electron-Spin Polarization in Low-Energy Electron Diffraction from Tungsten (001). Phys. Rev. Lett. 34, 1167 (1975).

149. Kirschner, J. \& Feder, R. Spin Polarization in Double Diffraction of Low-Energy Electrons from W(001): Experiment and Theory. Phys. Rev. Lett. 42, 1008 (1979). 
150. Oepen, H. P., Hnlich, K., Kirschner, J., Eyers, A., Schafers, F., Schnhense, G. \& Heinzmann, U. Experimental symmetry analysis of energy bands near critical points in Pt using spin- and momentum-resolved photoemission. Phys. Rev. B 31, 6846 (1985).

151. Knorren, R., Bennemann, K. H., Burgermeister, R. \& Aeschlimann, M. Dynamics of excited electrons in copper and ferromagnetic transition metals: Theory and experiment. Phys. Rev. B 61, 9427 (2000).

152. Aeschlimann, M., Bauer, M., Pawlik, S., Weber, W., Burgermeister, R., Oberli, D. \& Siegmann, H. C. Ultrafast Spin-Dependent Electron Dynamics in fcc Co. Phys. Rev. Lett. 79, 5158 (1997).

153. Aeschlimann, M., Burgermeister, R., Pawlik, S., Bauer, M., Oberli, D. \& Weber, W. Spin-dependent electron dynamics investigated by means of time- and spin-resolved photoemission. J. Electron Spectrosc. Relat. Phenom. 88-91, 179 (1998).

154. Schneider, H. C. et al. Energy-resolved electron spin dynamics at surfaces of p-doped GaAs. Phys. Rev. B 73, 081302 (2006).

155. Andreyev, O. et al. Spin-resolved two-photon photoemission study of the surface resonance state on $\mathrm{Co} / \mathrm{Cu}(001)$. Phys. Rev. B 74, 195416 (2006).

156. Unguris, J., Pierce, D. T. \& Celotta, R. J. Low-energy diffuse scattering electron-spin polarization analyzer. Rev. Sci. Instrum. 57, 1314 (1986).

157. Johnson, P. D. et al. Spin-polarized photoemission spectroscopy of magnetic surfaces using undulator radiation. Rev. Sci. Instrum. 63, 1902 (1992).

158. Pierce, D. T., Celotta, R. J., Kelley, M. H. \& Unguris, J. Electron spin polarization analyzers for use with synchrotron radiation. Nucl. Instrum. Methods A 266, 550 (1988).

159. Schnhense, G. \& Siegmann, H. C. Transmission of electrons through ferromagnetic material and applications to detection of electron spin polarization. Ann. Phys. (Leipzig) 505, 465 (1993).

160. Lassailly, Y., Drouhin, H.-J., van der Sluijs, A. J., Lampel, G. \& Marlire, C. Spindependent transmission of low-energy electrons through ultrathin magnetic layers. Phys. Rev. B 50, 13054 (1994).

161. Van der Sluijs, A., Drouhin, H. J., Lampel, G., Lassailly, Y. \& Marliere, C. Spindependent electron transmission through ultra-thin magnetic layers: towards highly discriminative, compact spin detectors. C. R. Acad. Sci., Paris 319, 753 (1994).

162. Tamura, E., Feder, R., Krewer, J., Kirby, R. E., Kisker, E., Garwin, E. L. \& King, F. K. Energy-dependence of inner potential in Fe from low-energy electron absorption (target current). Solid State Commun. 55, 543 (1985).

163. Tillmann, D., Thiel, R. \& Kisker, E. Very-low-energy spin-polarized electron diffraction from $\mathrm{Fe}(001)$. Z. Phys. B 77, 1 (1989). 
164. Hillebrecht, F. U., Jungblut, R. \& Kisker, E. Spin polarization of the metallic Fe 3s photoemission spectrum. Phys. Rev. Lett. 65, 2450 (1990).

165. Jungblut, R., Roth, C., Hillebrecht, F. U. \& Kisker, E. Spin-polarized electron spectroscopy as a combined chemical and magnetic probe. Surf. Sci. 269-270, 615 (1992).

166. Hammond, M. S., Fahsold, G. \& Kirschner, J. Absorption and elastic and inelastic reflection of spin-polarized low-energy electrons from Fe(110). Phys. Rev. B 45, 6131 (1992).

167. Fahsold, G., Hammond, M. S., Kirschner, J., Krewer, J. W. \& Feder, R. Spindependent electron reflection from Fe(1 10 ): Experiment and theory. Solid State Commun. 84, 541 (1992).

168. Hillebrecht, F. U. et al. High-efficiency spin polarimetry by very-low-energy electron scattering from $\mathrm{Fe}(100)$ for spin-resolved photoemission. Rev. Sci. Instrum. 73, 1229 (2002).

169. Bertacco, R., Onofrio, D. \& Ciccacci, F. A novel electron spin-polarization detector with very large analyzing power. Rev. Sci. Instrum. 70, 3572 (1999).

170. Bertacco, R., Marcon, M., Trezzi, G., Duo, L. \& Ciccacci, F. Spin and energy analysis of electron beams: Coupling a polarimeter based on exchange scattering to a hemispherical analyzer. Rev. Sci. Instrum. 73, 3867 (2002).

171. Bertacco, R., Merano, M. \& Ciccacci, F. Spin dependent electron absorption in $\mathrm{Fe}(001)-\mathrm{p}(1 \times 1) \mathrm{O}$ : A new candidate for a stable and efficient electron polarization analyzer. Appl. Phys. Lett. 72, 2050 (1998).

172. Bertacco, R. \& Ciccacci, F. Large spin asymmetry in electron absorption and reflection from oxidized single crystal Fe/MgO(001) films. Surf. Sci. 419, 265 (1999).

173. Bauer, E. Low energy electron microscopy. Rep. Prog. Phys. 895 (1994).

174. Graf, J., Jozwiak, C., Schmid, A. K., Hussain, Z. \& Lanzara, A. Mapping the spindependent electron reflectivity of Fe and Co ferromagnetic thin films. Phys. Rev. B 71, 144429 (2005).

175. Kurzawa, R., Kmper, K. P., Schmitt, W. \& Gntherodt, G. Spin-resolved photoemission study of in situ grown epitaxial Fe layers on W(110). Solid State Commun. 60, 777 (1986).

176. Getzlaff, M., Bansmann, J. \& Schnhense, G. Spin-resolved photoemission from physisorbed xenon on ferromagnetic surfaces: Evidence for magnetic interactions. Phys. Rev. Lett. 71, 793 (1993).

177. Bansmann, J., Lu, L., Getzlaff, M. \& Meiwes Broer, K. Magnetic properties of transition metal films and islands on W(110). Z. Phys. D 40, 570 (1997).

178. Scheunemann, T. et al. Quantum well resonances in ferromagnetic Co films. Solid State Commun. 104, 787 (1997). 
179. Zdyb, R. \& Bauer, E. Spin-Resolved Unoccupied Electronic Band Structure from Quantum Size Oscillations in the Reflectivity of Slow Electrons from Ultrathin Ferromagnetic Crystals. Phys. Rev. Lett. 88, 166403 (2002).

180. Citrin, P. H., Wertheim, G. K. \& Baer, Y. Surface-atom x-ray photoemission from clean metals: Cu, Ag, and Au. Phys. Rev. B 27, 3160 (1983).

181. Citrin, P. H. \& Wertheim, G. K. Photoemission from surface-atom core levels, surface densities of states, and metal-atom clusters: A unified picture. Phys. Rev. B 27, 3176 (1983).

182. Riffe, D. M., Wertheim, G. K. \& Citrin, P. H. Different core-hole lifetime and screening in the surface of W(110). Phys. Rev. Lett. 63, 1976 (1989).

183. Tanuma, S., Powell, C. J. \& Penn, D. R. Calculations of electorn inelastic mean free paths. II. Data for 27 elements over the $50-2000 \mathrm{eV}$ range. Surface and Interface Analysis 17, 911 (1991).

184. Kim, H. J., Vescovo, E., Heinze, S. \& Blgel, S. Surface electronic structure of Fe(110): the importance of surface resonances. Surf. Sci. 478, 193 (2001).

185. Sinkovic, B., Shekel, E. \& Hulbert, S. L. Spin-resolved iron surface density of states. Phys. Rev. B 52, R8696 (1995).

186. LaShell, S., McDougall, B. A. \& Jensen, E. Spin Splitting of an Au(111) Surface State Band Observed with Angle Resolved Photoelectron Spectroscopy. Phys. Rev. Lett. 77, 3419 (1996).

187. Nicolay, G., Reinert, F., Hfner, S. \& Blaha, P. Spin-orbit splitting of the L-gap surface state on $\mathrm{Au}(111)$ and $\mathrm{Ag}(111)$. Phys. Rev. B 65, 033407 (2001).

188. Henk, J., Ernst, A. \& Bruno, P. Spin polarization of the L-gap surface states on $\mathrm{Au}(111)$. Phys. Rev. B 68, 165416 (2003).

189. Hoesch, M. et al. Spin structure of the Shockley surface state on Au ( 111 ). Phys. Rev. B 69, 241401 (2004).

190. Bauer, E., Poppa, H., Todd, G. \& Davis, P. R. The adsorption and early stages of condensation of $\mathrm{Ag}$ and $\mathrm{Au}$ on $\mathrm{W}$ single-crystal surfaces. J. Appl. Phys. 48, 3773 (1977).

191. Shikin, A. M., Rader, O., Prudnikova, G. V., Adamchuk, V. K. \& Gudat, W. Quantum well states of sp- and d-character in thin Au overlayers on W(110). Phys. Rev. B 65, 075403- (2002).

192. Dedkov, Y. S., Fonin, M. \& Laubschat, C. A possible source of spin-polarized electrons: The inert graphene/Ni(111) system. Appl. Phys. Lett. 92, 052506 (2008).

193. Dedkov, Y. S., Fonin, M., Rudiger, U. \& Laubschat, C. Graphene-protected iron layer on Ni(111). Appl. Phys. Lett. 93, 022509 (2008).

194. Jonker, G. H. \& Van Santen, J. H. Ferromagnetic compounds of manganese with perovskite structure. Physica 16, 337 (1950). 
195. Van Santen, J. H. \& Jonker, G. H. Electrical conductivity of ferromagnetic compounds of manganese with perovskite structure. Physica 16, 599 (1950).

196. Jin, S., Tiefel, T. H., McCormack, M., Fastnacht, R. A., Ramesh, R. \& Chen, L. H. Thousandfold Change in Resistivity in Magnetoresistive La-Ca-Mn-O Films. Science 264, 413 (1994).

197. Jin, S., McCormack, M., Tiefel, T. H. \& Ramesh, R. Colossal magnetoresistance in La-Ca-Mn-O ferromagnetic thin films (invited). J. Appl. Phys. 76, 6929 (1994).

198. Ramirez, A. P. Colossal magnetoresistance. Journal of Physics: Condensed Matter 9, 8171 (1997).

199. Coey, J. M. D., Viret, M. \& von Molnr, S. Mixed-valence manganites. Adv. Phys. 48, 167 (1999).

200. Tokura, Y. \& Tomioka, Y. Colossal magnetoresistive manganites. Journal of Magnetism and Magnetic Materials 200, 1 (1999).

201. Salamon, M. B. \& Jaime, M. The physics of manganites: Structure and transport. Rev. Mod. Phys. 73, 583 (2001).

202. Zener, C. Interaction between the d-Shells in the Transition Metals. II. Ferromagnetic Compounds of Manganese with Perovskite Structure. Phys. Rev. 82, 403 (1951).

203. Anderson, P. W. \& Hasegawa, H. Considerations on Double Exchange. Phys. Rev. 100, 675 (1955).

204. de Gennes, P. G. Effects of Double Exchange in Magnetic Crystals. Phys. Rev. 118, 141 (1960).

205. Soulen, R. J., J. et al. Measuring the Spin Polarization of a Metal with a Superconducting Point Contact. Science 282, 85 (1998).

206. Ji, Y. et al. Determination of the Spin Polarization of Half-Metallic $\mathrm{CrO}_{2}$ by Point Contact Andreev Reflection. Phys. Rev. Lett. 86, 5585 (2001).

207. Mitchell, J. F., Argyriou, D. N., Berger, A., Gray, K. E., Osborn, R. \& Welp, U. Spin, charge, and lattice states in layered magnetoresistive oxides. J. Phys. Chem. B 105, 10731 (2001).

208. Jozwiak, C. et al. Bilayer splitting and c-axis coupling in CMR bilayer manganites (2008). ArXiv:0806.2120v1, in review, Phys. Rev. B.

209. Huang, X. Y., Mryasov, O. N., Novikov, D. L. \& Freeman, A. J. Electronic and magnetic properties of layered colossal magnetoresistive oxides: $\mathrm{La}_{1+2 x} \mathrm{Sr}_{2-2 x} \mathrm{Mn}_{2} \mathrm{O}_{7}$. Phys. Rev. B 62, 13318 (2000).

210. Moritomo, Y., Asamitsu, A., Kuwahara, H. \& Tokura, Y. Giant magnetoresistance of manganese oxides with a layered perovskite structure. Nature 380, 141 (1996).

211. Dessau, D. S. et al. k-dependent electronic structure, a large "ghost" Fermi surface, and a pseudogap in a layered magnetoresistive oxide. Phys. Rev. Lett. 81, 192 (1998). 
212. Chuang, Y. D., Gromko, A. D., Dessau, D. S., Kimura, T. \& Tokura, Y. Fermi surface nesting and nanoscale fluctuating charge/orbital ordering in colossal magnetoresistive oxides. Science 292, 1509 (2001).

213. Mannella, N. et al. Polaron coherence condensation as the mechanism for colossal magnetoresistance in layered manganites. Phys. Rev. B 76, 233102 (2007).

214. Park, J.-H., Vescovo, E., Kim, H.-J., Kwon, C., Ramesh, R. \& Venkatesan, T. Direct evidence for a half-metallic ferromagnet. Nature 392, 794 (1998).

215. Park, J.-H., Vescovo, E., Kim, H.-J., Kwon, C., Ramesh, R. \& Venkatesan, T. Magnetic Properties at Surface Boundary of a Half-Metallic Ferromagnet $\mathrm{La}_{0.7} \mathrm{Sr}_{0.3} \mathrm{MnO}_{3}$. Phys. Rev. Lett. 81, 1953 (1998).

216. Hochstrasser, M., Tobin, J. G., Rotenberg, E. \& Kevan, S. D. Spin-Resolved Photoemission of Surface States of W(110)-(1×1)H. Phys. Rev. Lett. 89, 216802 (2002).

217. Krupin, O. et al. Rashba effect at magnetic metal surfaces. Phys. Rev. B 71, 201403 (2005).

218. Nicolay, G., Reinert, F., Forster, F., Ehm, D., Schmidt, S., Eltner, B. \& Hfner, S. About the stability of noble-metal surfaces during VUV-photoemission experiments. Surf. Sci. 543, 47 (2003).

219. Forster, F., Hufner, S. \& Reinert, F. Rare Gases on Noble-Metal Surfaces:\&nbsp; An Angle-Resolved Photoemission Study with High Energy Resolution. J. Phys. Chem. B 108, 14692 (2004).

220. Moreschini, L., Ghiringhelli, G., Larsson, K., Veit, U. \& Brookes, N. B. A time-offlight-Mott apparatus for soft x-ray spin resolved photoemission on solid samples. Rev. Sci. Instrum. 79, 033905 (2008).

221. Rotenberg, E., Chung, J. W. \& Kevan, S. D. Spin-Orbit Coupling Induced Surface Band Splitting in Li/W(110) and Li/Mo(110). Phys. Rev. Lett. 82, 4066 (1999).

222. Ast, C. R. et al. Giant Spin Splitting through Surface Alloying. Phys. Rev. Lett. 98, 186807 (2007).

223. Shikin, A. M. et al. Origin of Spin-Orbit Splitting for Monolayers of Au and Ag on $\mathrm{W}(110)$ and $\mathrm{Mo}(110)$. Phys. Rev. Lett. 100, 057601 (2008).

224. Varykhalov, A. et al. Electronic and Magnetic Properties of Quasifreestanding Graphene on Ni. Phys. Rev. Lett. 101, 157601 (2008).

225. Zhou, S. Y. et al. Substrate-induced bandgap opening in epitaxial graphene. Nat. Mater. 6, 770 (2007).

226. Zhou, S. Y., Siegel, D. A., Fedorov, A. V. \& Lanzara, A. Metal to Insulator Transition in Epitaxial Graphene Induced by Molecular Doping. Phys. Rev. Lett. 101, 086402 (2008).

227. Bostwick, A., Ohta, T., Seyller, T., Horn, K. \& Rotenberg, E. Quasiparticle dynamics in graphene. Nat. Phys. 3, 36 (2007). 
228. Fiebig, M., Miyano, K., Tomioka, Y. \& Tokura, Y. Visualization of the Local InsulatorMetal Transition in $\operatorname{Pr}_{0.7} \mathrm{Ca}_{0.3} \mathrm{MnO}_{3}$. Science 280, 1925 (1998). 